\title{
WestVirginiaUniversity
}

THE RESEARCH REPOSITORY @ WVU

Graduate Theses, Dissertations, and Problem Reports

1998

\section{School bus crashworthiness}

Ganesh R. Panneer

West Virginia University

Follow this and additional works at: https://researchrepository.wvu.edu/etd

\section{Recommended Citation}

Panneer, Ganesh R., "School bus crashworthiness" (1998). Graduate Theses, Dissertations, and Problem Reports. 902.

https://researchrepository.wvu.edu/etd/902

This Thesis is protected by copyright and/or related rights. It has been brought to you by the The Research Repository @ WVU with permission from the rights-holder(s). You are free to use this Thesis in any way that is permitted by the copyright and related rights legislation that applies to your use. For other uses you must obtain permission from the rights-holder(s) directly, unless additional rights are indicated by a Creative Commons license in the record and/ or on the work itself. This Thesis has been accepted for inclusion in WVU Graduate Theses, Dissertations, and Problem Reports collection by an authorized administrator of The Research Repository @ WVU. For more information, please contact researchrepository@mail.wvu.edu. 


\title{
School Bus Crashworthiness
}

\author{
Ganesh R. Panneer
}

Thesis submitted to the faculty of the College of Engineering and Mineral Resources at West Virginia University in partial fulfillment of the requirements for the degree of

\author{
Master of Science \\ in \\ Mechanical Engineering \\ Gregory Thompson, Ph.D., Chair \\ James Smith, Ph.D \\ Victor Mucino, Ph.D. \\ May 22, 1998 \\ Morgantown, West Virginia
}

Keywords: Crashworthiness, School Bus, Numerical Simulation 


\title{
School Bus Crashworthiness
}

\author{
Ganesh R. Panneer
}

\section{(ABSTRACT)}

Numerical simulation of automotive crashes play an important role in reducing the cost and time taken for predicting the results of a collision. Computer simulation of a vehicle requires that the vehicle structure be modeled in a finite element package by discretizing the geometry into a number of elements.

Every day thousands of children travel to school and school related events by bus. Not necessarily all the journeys are short and safely driven. The potential for serious injuries is possible in the event of a crash. The severity of injury in an offset frontal impact is higher than the full frontal impact, because of the offset in the principal direction of the impact force.

A finite element model of a school bus was created in I-DEAS Master Series. The body structure was modeled with the rib structure and the body skin. The chassis was modeled with engine, gearbox, drive train, and axles. The body structure was attached to the chassis to create a complete finite element model of the bus. IDEADYN was used as a translator to write a LS-DYNA3D input file. Full frontal and offset frontal impacts were simulated in LS-DYNA3D with an initial velocity of $56 \mathrm{~km} / \mathrm{hr}$ against a rigid wall. Since hourglassing energy was high in the previous results, a higher order integration was done for all the thin shell elements with Hughes-Liu SR thin shell elements. LS-TAURUS was used to post process the results obtained from the simulation. The results from the analysis included nodal displacement, velocity and accelerations, energy absorption, rigid wall forces, and occupant intrusion. The results from the two cases, with and without hourglassing energy, were compared. 


\section{Acknowledgements}

I would like to take this opportunity to express my deepest gratitude to my research advisor, Dr. Gregory J. Thompson, for his constant support, enthusiasm and motivation. His encouragement and guidance have helped me achieve my academic goals and at the same time made me a more complete person.

This gives me a platform to thank Dr. James E. Smith who by his timely support provided me with one of the best environments to carry out research in the area of crashworthiness. His guidance helped me overcome many of the problems I faced during the course of my thesis work.

I would also like to thank my committee member Dr. Victor H. Mucino for his guidance and support during my research work.

Thanks are also due to Joanna Davis-Swing and Jack Connolly for all the help extended. 


\section{Table of Contents}

Acknowledgements....................................................................................................................................................... ii

Table of Contents .......................................................................................................................................................... iii

List of Figures ....................................................................................................................................................

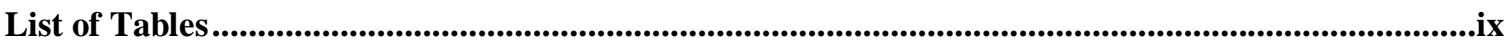

Nomenclature ..............................................................................................................................................................

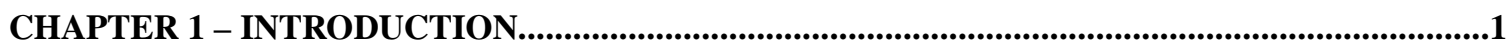

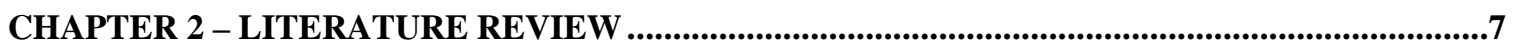

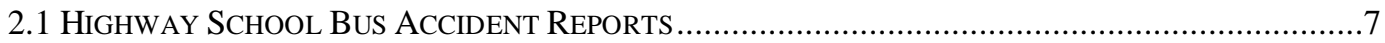

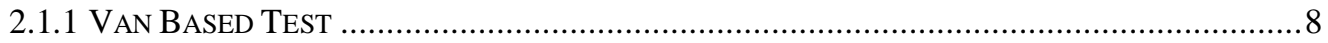

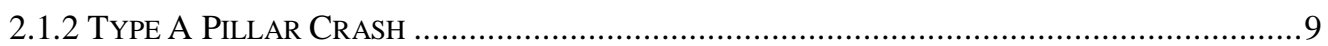

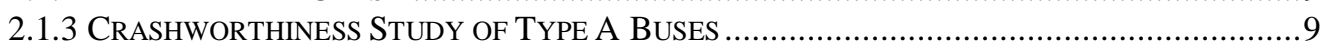

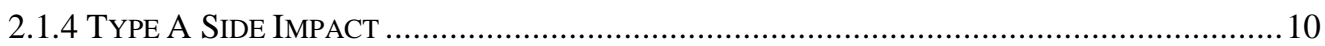

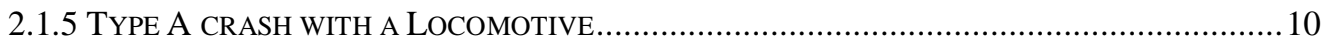

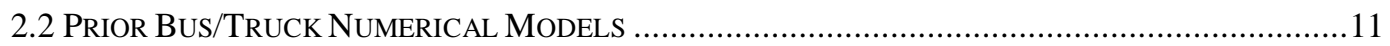

2.3 Modeling ApProaches to Vehicle CrashworthinesS Simulation .................................13

2.4 Software Packages for Vehicle CrashworthineSS SimUlation ....................................35

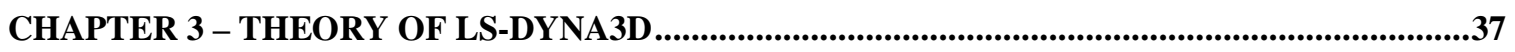

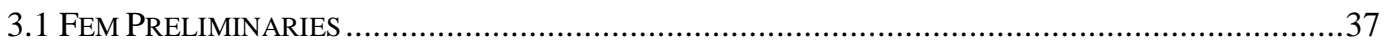

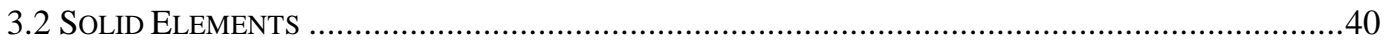

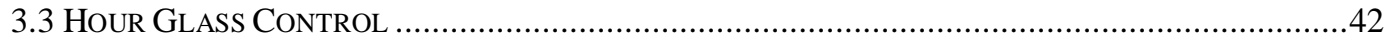

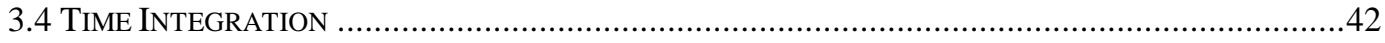

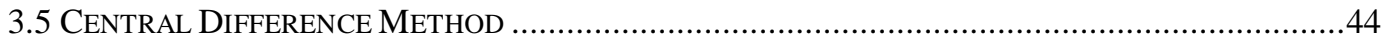

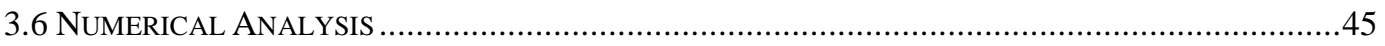

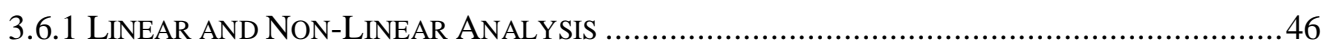

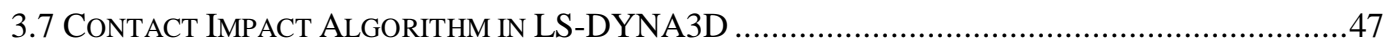

CHAPTER 4 - FINITE ELEMENT MODEL OF THE BUS ..................................................................50

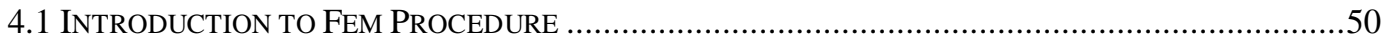

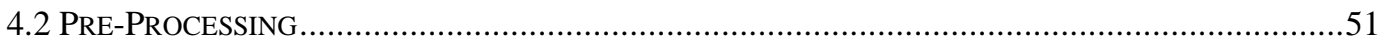

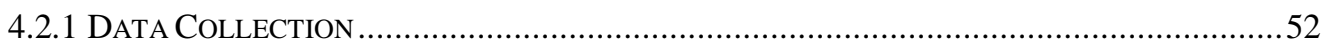

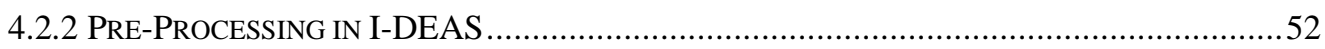

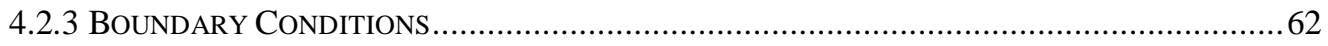

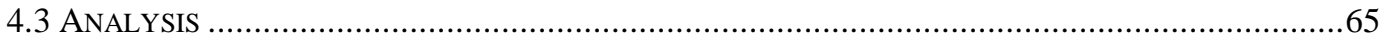

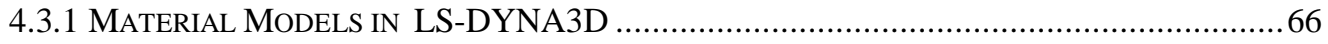

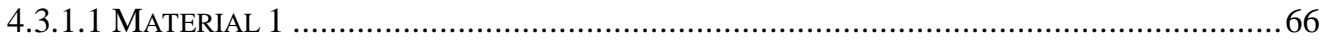

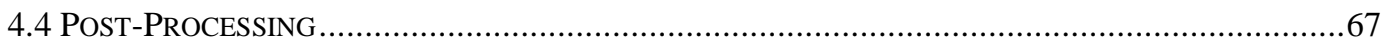

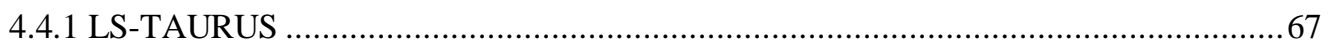

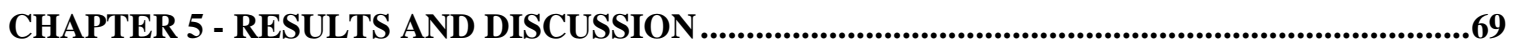

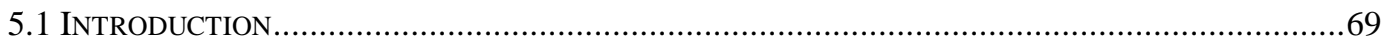

5.2 Full FRONTAL IMPACT WITH BELYTSCHKO -TSAY THIN SHELL ELEMENTS ............................72 
5.3 OfFSET FrONTAL IMPACT WITH BELYTSCHKO -TSAY THIN SHELL ELEMENTS ........................95

5.4 HighER ORDER INTEGRATION FOR THIN SHELL ELEMENTS......................................... 116

5.5 Full Frontal IMPACt With HugheS-Liu SR Thin SHELl ElEMENTS............................. 117

5.6 OfFSET Frontal ImPaCt With HugheS-Liu SR Thin SHELl ElEMENTS ......................... 142

CHAPTER 6 -CONCLUSIONS AND RECOMMENDATIONS ..........................................165

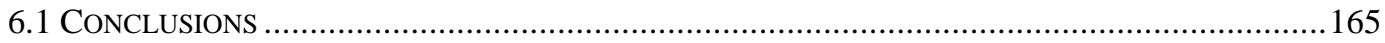

CHAPTER - 8 REFERENCES ......................................................................................171

APPENDIX A - FMVSS FOR SCHOOL BUS.............................................................................176

APPENDIX B - CONDENSED LS-DYNA3D INPUT FILE ......................................................177

APPENDIX C - YIELD STRESS OF MATERIALS ....................................................................188

VITA

APPROVAL OF EXAMINING COMMITTEE...................................................................................190 


\section{List of Figures}

Fig 2.1 Influence of mesh density on displacement................................................................ 16

Fig 2.2 Graph showing the deceleration force varying linearly with time....................................... 18

Fig 2.3 Deformation of the Suzuki Sidekick finite element model. ...................................................21

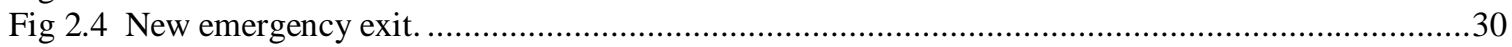

Fig 3.1 Displacement of a body from position $\mathrm{B}$ at time $\mathrm{t}=0$ to position $\mathrm{b}$ at time $\mathrm{t}=\mathrm{T}$. ......................3 37

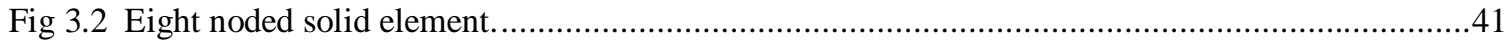

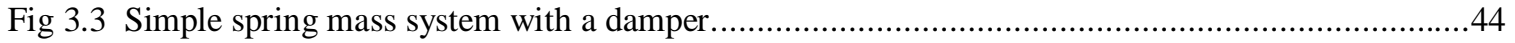

Fig 3.4 Free body diagram of the spring mass system..............................................................44

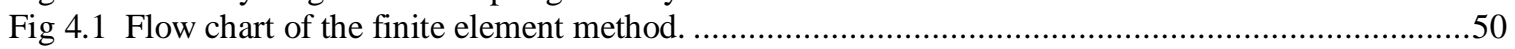

Fig 4.2 Frame structure of the bus with springs and fuel tank. ....................................................53

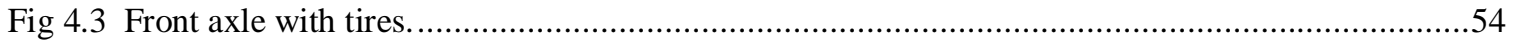

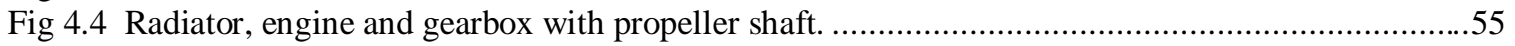

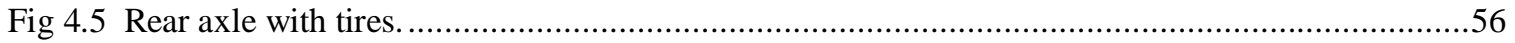

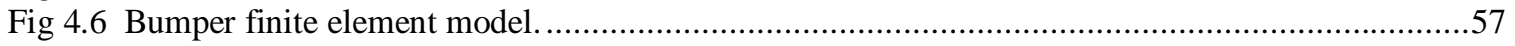

Fig 4.7 Fire wall finite element model....................................................................................5

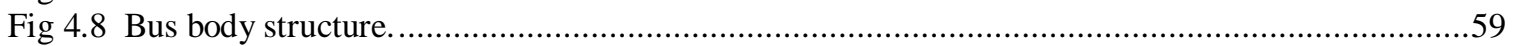

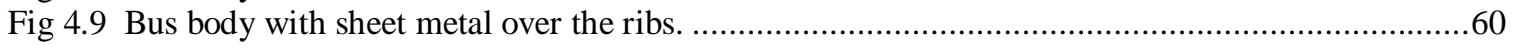

Fig 4.10 Complete finite element model of the bus with different parts assembled.............................61

Fig 4.11 Flow chart for file translation from I-DEAS to LS-DYNA3D. .....................................64

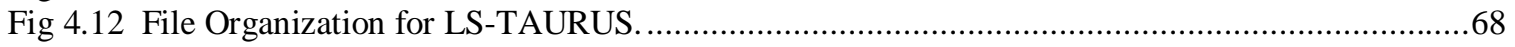

Fig 5.1 Full finite element model of the school bus with coordinate system. ..................................71

Fig 5.2 Front view at time $=0 \mathrm{msec}$ of the bus subjected to a full frontal impact at a velocity of 56

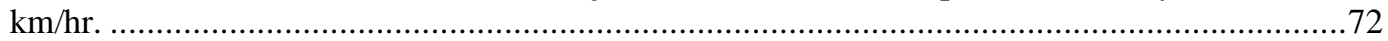

Fig 5.3 Front view at time $=9 \mathrm{msec}$ of the bus subjected to a full frontal impact at a velocity of 56

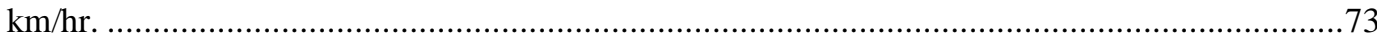

Fig 5.4 Front view at time $=18 \mathrm{msec}$ of the bus subjected to a full frontal impact at a velocity of

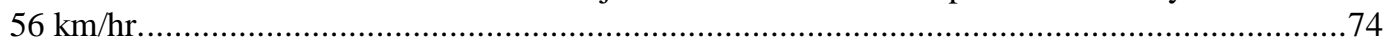

Fig 5.5 Front view at time $=27 \mathrm{msec}$ of the bus subjected to a full frontal impact at a velocity of $56 \mathrm{~km} / \mathrm{hr}$.

Fig 5.6 Front view at time $=36 \mathrm{msec}$ of the bus subjected to a full frontal impact at a velocity of

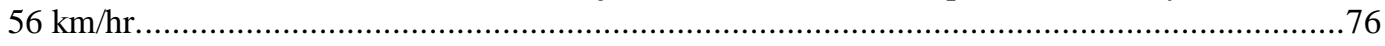

Fig 5.7 Front view at time $=45 \mathrm{msec}$ of the bus subjected to a full frontal impact at a velocity of

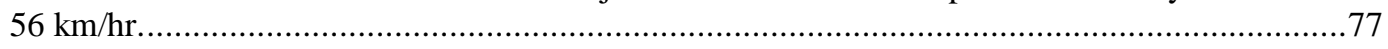

Fig 5.8 Front view at time $=54 \mathrm{msec}$ of the bus chassis subjected to a full frontal impact at a velocity of $56 \mathrm{~km} / \mathrm{hr}$.

Fig 5.9 Front view at time $=72 \mathrm{msec}$ of the bus chassis subjected to a full frontal impact at a velocity of $56 \mathrm{~km} / \mathrm{hr}$.

Fig 5.10 Front view at time $=90 \mathrm{msec}$ of the bus subjected to a full frontal impact at a velocity of

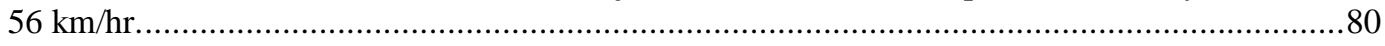

Fig 5.11 Figure showing the side view of the bus at time $=45 \mathrm{msec}$ for full frontal impa $7 \mathrm{ct} . \ldots \ldots \ldots \ldots \ldots \ldots . . . . . . .11$

Fig 5.12 Figure showing the top view of the bus at time $=45 \mathrm{msec}$ for full frontal impact...................82

Fig 5.13 Figure showing the deformation of the frame at time $=90 \mathrm{msec}$ for a full frontal impact...........82

Fig 5.14 Figure showing the VonMises stress plot in the front end of the frame at time $=45 \mathrm{msec} \ldots \ldots \ldots . . .83$

Fig 5.15 Relative displacement between the frame and the front tire ..........................................8

Fig 5.16 Relative displacement between the front tire and the rear tire (pitching).............................85

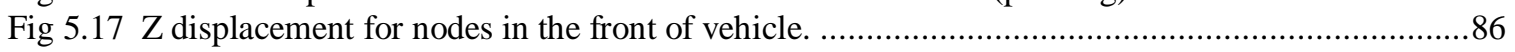

Fig $5.18 \mathrm{Z}$ displacement for nodes in the middle of vehicle. .......................................................86

Fig $5.19 \mathrm{Z}$ displacement for nodes in the rear of vehicle.........................................................8

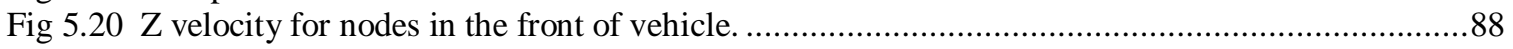

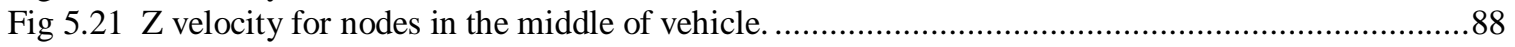




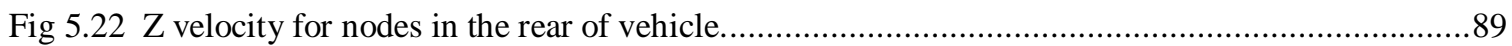

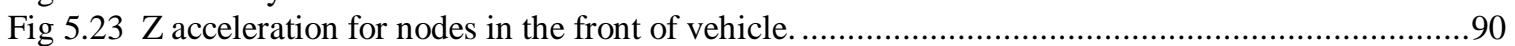

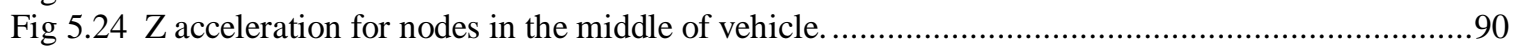

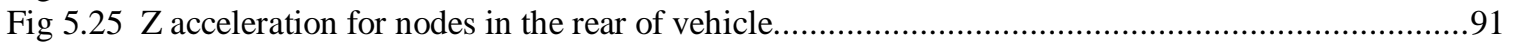

Fig 5.26 Z rigid body velocity for materials(Refer to Table 5.1 for table of materials).............................92

Fig $5.27 \mathrm{Z}$ rigid body velocity for a bus subjected to a full frontal impact at $56 \mathrm{~km} / \mathrm{hr}$..........................92

Fig 5.28 Internal energy for materials (refer to Table 5.1 for table of materials).....................................93

Fig 5.29 Internal, kinetic, and the total energies for a full frontal impact...........................................94

Fig 5.30 Total internal energy distribution at time $=90 \mathrm{msec}$ of the bus subjected to a full frontal

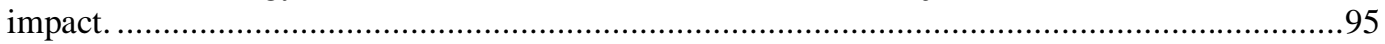

Fig 5.31 Front view at time $=9 \mathrm{msec}$ of the bus subjected to an offset frontal impact at a velocity

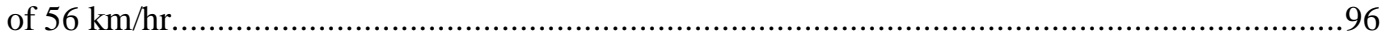

Fig 5.32 Front view at time $=18 \mathrm{msec}$ of the bus subjected to an offset frontal impact at a velocity

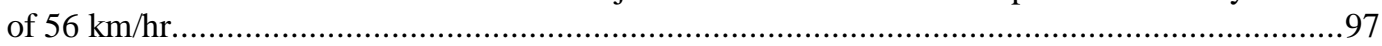

Fig 5.33 Front view at time $=27 \mathrm{msec}$ of the bus subjected to an offset frontal impact at a velocity of $56 \mathrm{~km} / \mathrm{hr}$

Fig 5.34 Front view at time $=36 \mathrm{msec}$ of the bus subjected to an offset frontal impact at a velocity

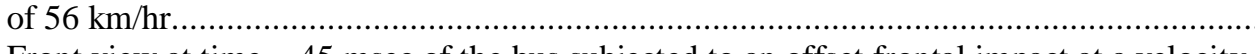

Fig 5.35 Front view at time $=45 \mathrm{msec}$ of the bus subjected to an offset frontal impact at a velocity

Fig 5.36 Front view at time $=54 \mathrm{msec}$ of the bus subjected to an offset frontal impact at a velocity

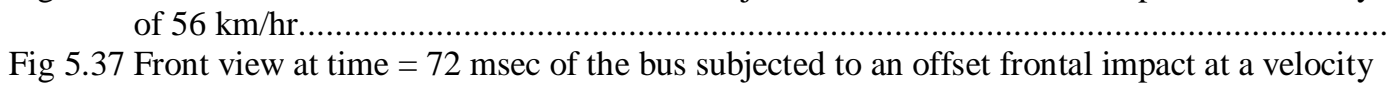

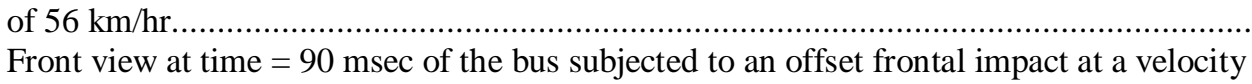

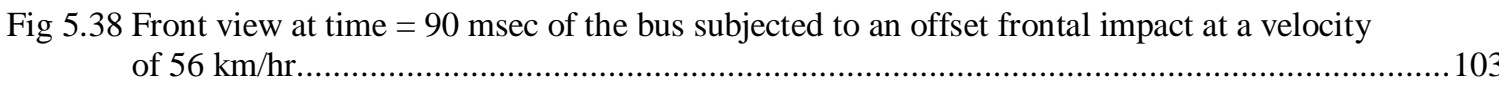

Fig 5.39 Side view at time $=45 \mathrm{msec}$ of the bus subjected to an offset frontal impact. ...........................104

Fig 5.40 Top view at time $=45 \mathrm{msec}$ of the bus subjected to an offset frontal impact.............................104

Fig 5.41 Relative displacement between the nodes in the right and the left tires in front axle..................105

Fig 5.42 Relative displacement between the front tire and the rear tire.............................................106

Fig 5.43 Relative displacement between the nodes in the fire wall and the right tire.............................106

Fig 5.44 Z displacement for the nodes in the front of the vehicle. .....................................................107

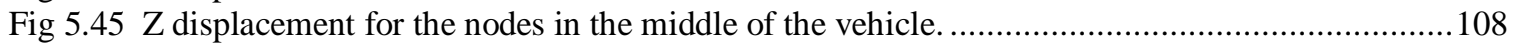

Fig 5.46 Z displacement for the nodes in the rear of the vehicle........................................................108

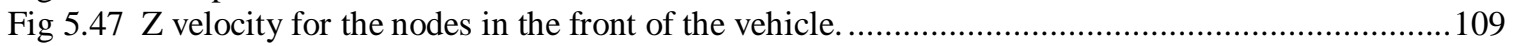

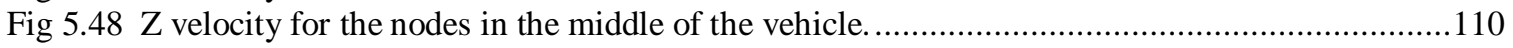

Fig $5.49 \mathrm{Z}$ velocity for the nodes in the rear of the vehicle. ............................................................110

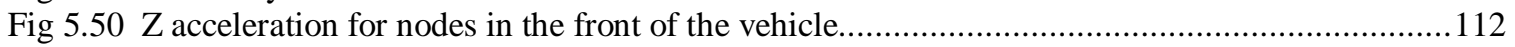

Fig $5.51 \mathrm{Z}$ acceleration for the nodes in the middle of the vehicle..................................................112

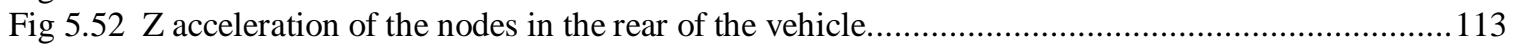

Fig 5.53 Z rigid body velocity for materials (Refer to Table 5.1 for table of materials)...........................114

Fig 5.54 Internal energy absorbed by materials (Refer to Table 5.1 for table of materials)......................115

Fig 5.55 Kinetic, internal and the total energies for an offset frontal impact.......................................116

Fig 5.56 Total internal energy distribution at time $=90 \mathrm{msec}$ of the bus subjected to an offset

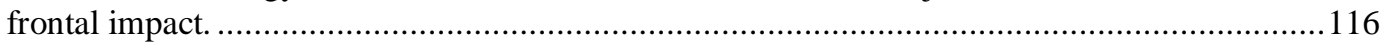

Fig 5.57 Front view at time $=0 \mathrm{msec}$ of the bus subjected to a full frontal impact with Hughes-Liu

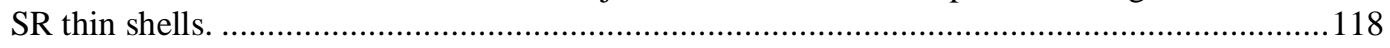

Fig 5.58 Front view at time $=9 \mathrm{msec}$ of the bus subjected to a full frontal impact with Hughes-Liu

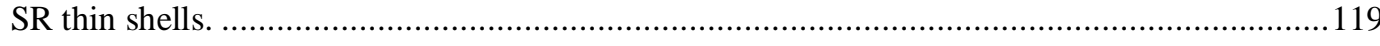

Fig 5.59 Front view at time $=18 \mathrm{msec}$ of the bus subjected to a full frontal impact with Hughes-

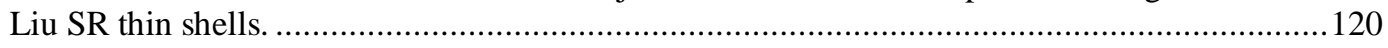

Fig 5.60 Front view at time $=27 \mathrm{msec}$ of the bus subjected to a full frontal impact with Hughes-

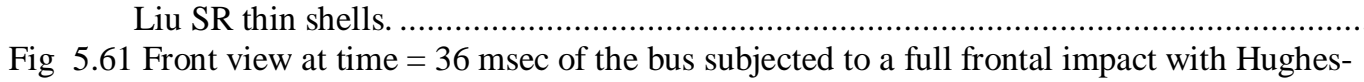
Liu SR thin shells. 
Fig 5.62 Front view at time $=45 \mathrm{msec}$ of the chassis of the bus subjected to a full frontal impact with Hughes-Liu SR thin shells.

Fig 5.63 VonMises stress plot in the front end of the frame at time $=45 \mathrm{msec}$ for a full frontal impact with Hughes-Liu SR thin shells.

Fig 5.64 Side view at time $=45 \mathrm{msec}$ of the bus subjected to a full frontal impact with HughesLiu SR thin shells.

Fig 5.65 Top view at time $=45 \mathrm{msec}$ of the bus subjected to a full frontal impact with Hughes-Liu SR thin shells.

Fig 5.66 Relative displacement between the hood and the frame for a full frontal impact with Hughes-Liu SR thin shells.

Fig 5.67 $\mathrm{Z}$ relative displacement between the frame and the front tire for a full frontal impact with Hughes-Liu SR thin shells.

Fig 5.68 $\mathrm{Z}$ displacement for the nodes in the front of the vehicle for full frontal impact with Hughes-Liu SR thin shells.

Fig 5.69 $\mathrm{Z}$ displacement for the nodes in the middle of the vehicle for full frontal impact with Hughes-Liu SR thin shells.

Fig 5.70 $\mathrm{Z}$ displacement for the nodes in the rear of the vehicle for full frontal impact with Hughes-Liu SR thin shells.

Fig 5.71 Z velocity for the nodes in the front of the vehicle for full frontal impact with HughesLiu SR thin shells.

Fig 5.72 Z velocity for the nodes in the middle of the vehicle for full frontal impact with HughesLiu SR thin shells.

Fig 5.73 $\mathrm{Z}$ velocity for the nodes in the rear of the vehicle for full frontal impact with Hughes-Liu SR thin shells.

Fig 5.74 $\mathrm{Z}$ acceleration for the nodes in the front of the vehicle for full frontal impact with Hughes-Liu SR thin shells.

Fig 5.75 $\mathrm{Z}$ acceleration for the nodes in the middle of the vehicle for full frontal impact with Hughes-Liu SR thin shells.

Fig 5.76 $\mathrm{Z}$ acceleration for the nodes in the rear of the vehicle for full frontal impact with HughesLiu SR thin shells.

Fig 5.77 X rigid body velocities of materials for a full frontal impact with Hughes-Liu SR thin shells.

Fig 5.78 Y rigid body velocities of materials for a full frontal impact with Hughes-Liu SR thin shells.

Fig 5.79 Z rigid body velocities of materials for a full frontal impact with Hughes-Liu SR thin shells.

Fig 5.80 Total rigid body velocity for materials for a full frontal impact with Hughes-Liu SR thin

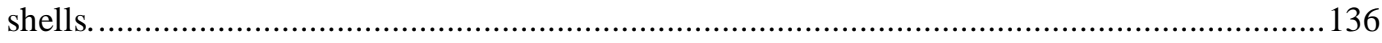

Fig 5.81 Global X rigid body velocity for a full frontal impact with Hughes-Liu SR thin shell. ............137

Fig 5.82 Global Y rigid body velocity for a full frontal impact with Hughes-Liu SR thin shell. ............138

Fig 5.83 Global Z rigid body velocity for a full frontal impact with Hughes-Liu SR thin shell. .............138

Fig 5.84 Global total rigid body velocity for a full frontal impact with Hughes-Liu SR thin shells.........139

Fig 5.85 Material internal energy absorption for a full frontal impact with Hughes-Liu SR thin shells.

Fig 5.86 Global energies for a full frontal impact with Hughes-Liu SR thin shells.

Fig 5.87 Total internal energy distribution at time $=45 \mathrm{msec}$ of the bus subjected to a full frontal impact with Hughes-Liu SR thin shells.

Fig 5.88 Front view at time $=0 \mathrm{msec}$ of the bus subjected to an offset frontal impact with HughesLiu SR thin shells.

Fig 5.89 Front view at time $=9 \mathrm{msec}$ of the bus subjected to an offset frontal impact with HughesLiu SR thin shells.

Fig 5.90 Front view at time $=18 \mathrm{msec}$ of the bus subjected to an offset frontal impact with Hughes-Liu SR thin shells.

Fig 5.91 Front view at time $=27 \mathrm{msec}$ of the bus subjected to an offset frontal impact with Hughes-Liu SR thin shells. 
Fig 5.92 Front view at time $=36 \mathrm{msec}$ of the bus subjected to an offset frontal impact with Hughes-Liu SR thin shells.

Fig 5.93 Front view at time $=45 \mathrm{msec}$ of the bus subjected to an offset frontal impact with Hughes-Liu SR thin shells.

Fig 5.94 Front view at time $=54 \mathrm{msec}$ of the bus subjected to an offset frontal impact with

Hughes-Liu SR thin shells.

Fig 5.95 Side view at time $=54 \mathrm{msec}$ of the bus subjected to an offset frontal impact with HughesLiu SR thin shells.

Fig 5.96 Top view at time $=54 \mathrm{msec}$ of the bus subjected to an offset frontal impact with HughesLiu SR thin shells.

Fig 5.97 Relative displacement between the right wheel and the left wheel (axle rotation) for an offset frontal impact with Hughes-Liu SR thin shell elements.

Fig 5.98 Relative displacement between the front tires and the rear tires (pitching) for an offset frontal impact with Hughes-Liu SR thin shell elements.

Fig 5.99 Z displacement for the nodes in the front of the vehicle for an offset frontal impact with Hughes-Liu SR thin shells.

Fig 5.100 Z displacement for the nodes in the middle of the vehicle for an offset frontal impact with Hughes-Liu SR thin shells.

Fig 5.101 Z displacement for the nodes in the rear of the vehicle for an offset frontal impact with Hughes-Liu SR thin shells.

Fig 5.102 Z velocity for the nodes in the front of the vehicle for an offset frontal impact with Hughes-Liu SR thin shells.

Fig 5.103 Z velocity for the nodes in the middle of the vehicle for an offset frontal impact with Hughes-Liu SR thin shells.

Fig 5.104 Z velocity for the nodes in the rear of the vehicle for an offset frontal impact with Hughes-Liu SR thin shells.

Fig 5.105 Z acceleration for the nodes in the front of the vehicle for an offset frontal impact with Hughes-Liu SR thin shells.

Fig 5.106 Z acceleration for the nodes in the middle of the vehicle for an offset frontal impact with Hughes-Liu SR thin shells.

Fig 5.107 $\mathrm{Z}$ acceleration for the nodes in the rear of the vehicle for an offset frontal impact with Hughes-Liu SR thin shells.

Fig 5.108 Material rigid body velocity of parts for an offset frontal impact with Hughes-Liu SR thin shells.

Fig 5.109 Global X rigid body velocity of the bus for an offset frontal impact with Hughes-Liu SR thin shells.

Fig 5.110 Global Y rigid body velocity of the bus for an offset frontal impact with Hughes-Liu SR thin shells.

Fig 5.111 Global Z rigid body velocity of the bus for an offset frontal impact with Hughes-Liu SR thin shells.

Fig 5.112 Global total rigid body velocity of the bus for an offset frontal impact with Hughes-Liu SR thin shells.

Fig 5.113 Material internal energy of parts for an offset frontal impact with Hughes-Liu SR thin shells.

Fig 5.114 Internal, kinetic and total energies for an offset frontal impact with Hughes-Liu SR thin shells.

Fig 5.115 Total internal energy distribution at time $=45 \mathrm{msec}$ of the bus subjected to an offset frontal impact with Hughes-Liu SR thin shells.

Fig 6.1 Instability in the total energy of the system with high hour glassing. 


\section{List of Tables}

Table 3.1 Operation count for hourglassing types...........................................................42

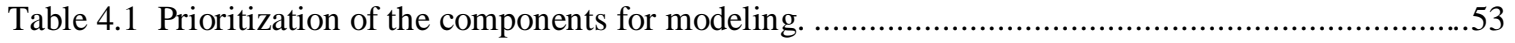

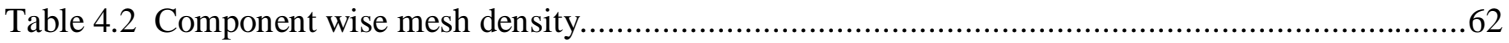

Table 4.3 Control switches for LS-DYNA3D......................................................................66

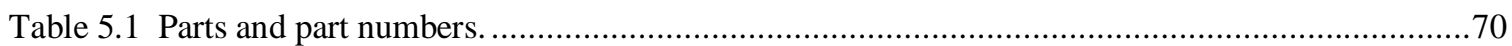




\section{Nomenclature}

$n_{j}-\quad$ Normal outward unit vector of boundary element

$\ddot{x}_{\mathrm{i}}-\quad$ Acceleration $\left[\mathrm{m} / \mathrm{s}^{2}\right]$

$\mathrm{f}_{\mathrm{i}}$ - Body force $[\mathrm{N}]$

$\mathrm{v}_{\mathrm{i}}-\quad$ Initial velocity $[\mathrm{m} / \mathrm{s}]$

$\mathrm{x}_{\mathrm{i}}-\quad$ Current velocity $[\mathrm{m} / \mathrm{s}]$

$\mathrm{k}_{\mathrm{i}}-\quad$ Number of Nodes

c - Damping coefficient [N.s/m]

$\mathrm{k}$ - $\quad$ Linear stiffness $[\mathrm{N} / \mathrm{m}]$

$\mathrm{M}$ - Diagonal mass matrix $[\mathrm{Kg}]$

$\mathrm{P}^{\mathrm{n}}-\quad$ External body and force loads $[\mathrm{N}]$

$\mathrm{F}^{\mathrm{n}}$ - Stress divergence vector $[\mathrm{N}]$

$\mathrm{H}^{\mathrm{n}}$ - Hour glass resistance $[\mathrm{N}]$

$\mathrm{u}$ - Displacement [m]

ù - Velocity $[\mathrm{m} / \mathrm{s}]$

ü - Acceleration $\left[\mathrm{m} / \mathrm{sec}^{2}\right]$

$\sigma_{\mathrm{i}, \mathrm{j}}-$ Cauchy Stress $\left[\mathrm{N} / \mathrm{m}^{2}\right]$

Greek

$\rho$ - Density $\left[\mathrm{Kg} / \mathrm{m}^{3}\right]$

$\phi_{\mathrm{j}}{ }^{-} \quad$ Shape function

$\beta$ - $\quad$ Parametric coordinates

$\eta$ - Parametric coordinates

$\Gamma$ - Parametric coordinates 


\section{CHAPTER 1 - INTRODUCTION}

Every day thousands of children travel to school and school related events by bus. An example of a typical school bus is shown in Figure 1.1. Since 1985, 1478 people have

died in school bus related crashes, an average of 134 fatalities per year ${ }^{26}$. Eleven percent of these fatalities were the occupants of the school bus, an average of 14 deaths per year. Impacts to the front of the vehicle occur in 47 percent of fatal school bus related crashes, impacts to the rear of the vehicle occur in 7 percent of the crashes, and impact to the side of the school bus occur in 15 percent of the crashes. Extensive research in passenger car crashworthiness has improved automobile safety to the point where occupants in a family sedan involved in an accident at relatively low speed will almost likely survive with only minor injuries. However, school bus crashworthiness has received much less attention, resulting in fewer improvements in the design and analysis of school buses.

The model presented here is the finite element model of a school bus, which was studied for different crash scenarios and the numerical requirements. This model shows the deformation and energy flow on the school bus in the event of a crash. The different crash scenarios that were studied are the full frontal collision against a rigid wall and the offset frontal collision against a rigid wall. In the full frontal rigid wall collision, the rigid wall modeled is infinitely rigid and the wall extends from positive infinity to negative infinity in the directions perpendicular to the rigid wall normal. The rigid wall normal is parallel but in opposite direction to the velocity vector of the vehicle. In the offset frontal collision, the rigid wall extends from the bus front centerline past the right side. 


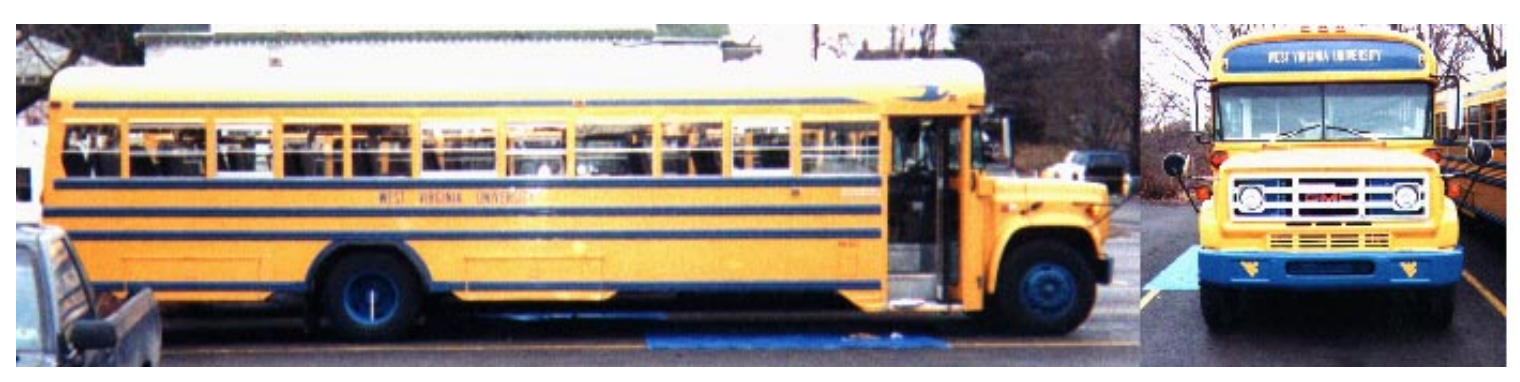

Fig 1.1 Side view and front view of a school bus.

Many improvements could reduce the risk of injury and death to children using school buses. This model presents cases that highlight areas for new designs for effective energy management in a crash. The model presented here is a crash analysis of a school bus; researchers can study this model and make improvements in necessary areas.

Simulating vehicle collisions on a computer reduces the cost and time necessary to predict the results of a collision. The experimental validation of the entire vehicle structure for crashworthiness is a complex an expensive task. Although the properly conducted experiments always yield better results, the cost incurred is much higher than the cost of the computer simulation. Lugt, Chen and Deshpande ${ }^{5}$ of General Motors Corp say that the prototype tests for vehicle crashworthiness are extremely expensive and do not reduce the development time and cost of the vehicle. Using crashworthiness simulation the outcome of the crash event can be predicted without conducting the actual test, and any change in the geometry or the materials of the design can be simulated and tested for crashworthiness scenarios. Also the various aspects of the new design, such as the energy flow and deformation, can be studied with the computer simulation of the crash. 
During the design stage of a vehicle, a numerical simulation and / or an experiment on a prototype can be done. Numerical modeling of the vehicle allows changes in the design to be incorporated at the cost of the CPU run, whereas the experimental crashworthiness test requires that each new prototype be manufactured and crashed. On the other hand, the final stage of design validation requires an experimental crashworthiness test, and the computer simulation is not an equivalent for this test. During the design stage of a vehicle, the structural configuration may change, and if an experimental prototype were to be built for each stage, it would be exorbitantly expensive. Igarashi ${ }^{4}$ of Suzuki Motor Co worked on the structural analysis of the Suzuki Sidekick at various stages of development and design where the numerical simulations were used at the earlier stages of design and testing and evaluation at the last stage of the development.

Each vehicle behaves differently under different collision events. Some of the vehicles may prove to be good against frontal impact but not against side impact and vice versa. The deformation and damage also differ from vehicle to vehicle. An accident reconstruction is possible by visiting the scene of an accident and hence deducing the events before the accident from the resulting wreck. Computer simulation allows not only visualization of the damage during the event, but also analysis of other factors like damage to the occupant and energy absorbed by different components of the vehicle.

The finite element method has been successfully used since the 1960's to predict the structural and dynamic behavior of beams and other members. This method of computer simulation reduced the time and the cost of experiments that would be conducted on these members to test their strength and other mechanical properties. 
Computer programs for crashworthiness were first developed around 1970. The finite element codes were written using a programming language and they were altered to the needs of the developer. They were run in main frame computers in those days, which took several hours to finish the computation. With the advent of supercomputers, even though the models are more complex, the computation has become easier and less expensive than it was previously.

The term crashworthiness by itself means the structural integrity of the member against crash and impact forces. Related to a vehicle, crashworthiness means the behavior of the vehicle in a collision with another vehicle or other object. The crashworthiness of a vehicle is measured by its ability to absorb energy and prevent any possible object intrusion causing an injury to the occupant. Crashworthiness studies currently use highspeed digital cameras to capture pictures since a high speed collision at a velocity of about 35 mph takes place only for about 60 to 80 milliseconds. Also, using of dummy models of different sizes has been extremely useful because researchers can study the impact on the human body and the possible injuries to them. Tests have been conducted with various automobile models for different kinds of collisions, and a large database had been developed by various research institutions which could be used for the study of collision in the happening of the same event elsewhere.

Computer simulation of a vehicle requires that the vehicle structure be modeled in a finite element package by discretizing the geometry into a number of elements. To save the time and cost of computing, it is absolutely necessary to model only the components that play a vital role in the crash scenario. Components that come into direct contact with the impacting structure play an important role in energy absorption; other components 
that have a substantially smaller mass compared to the mass of the vehicle can be neglected. Due to high deformation rates, the time step required for analyzing the event should be small which is computationally expensive.

\section{RESEARCH OBJECTIVES}

The research objectives of the following thesis are as follows.

1. Create a finite element model of the school bus in I-DEAS Master Series and use LS-DYNA3D to study the crashworthiness of the model in various collision events.

2. Run the analysis in the software for various crash scenarios.

3. Determine the numerical requirements.

4. Study the predicted behavior of the vehicle for deformation, energy distribution, energy flow, rigid wall energy absorption, and acceleration plots of various components.

5. Compare the results with the available data and standard crashworthiness analysis output.

Preprocessing of the model was created in I-DEAS Master Series ${ }^{15}$. Drawings were obtained from a manufacturer of a common 60-seat school bus, which is used for student transportation at West Virginia University and thousands of school districts throughout the United States. Along with the drawings, the dimensions of the components were 
measured by hand. The I-DEAS model information was translated into a LS-DYNA3D model with the translator IDEADYN ${ }^{12}$. After the solution phase, the preprocessing was performed in LS-TAURUS ${ }^{16}$ and the results were presented. 


\section{CHAPTER 2 - LITERATURE REVIEW}

Automobile manufacturers have used the finite element method in recent years to study crash characteristics of automobiles. This technique has helped them reduce the cost for testing each prototype that has to be manufactured during different stages of design. Possible occupant injuries can be determined by studying the deformation characteristics of the vehicle and the intrusion of parts into the occupant area after the crash. If there is a possibility of serious injury to the occupant, redesign can be carried out by simulation and also by testing the vehicle and comparing the results.

\subsection{HighWAY SCHOOL BuS ACCIDENT REPORTS}

Highway accident reports were obtained from National Technical Information Service $^{18}$ (NTIS) for accidents that involved school buses. These accident reports from NTIS give a detailed investigation of the accident scenario, the analysis of the accident and various factors that influenced the accident, findings, probable causes and recommendations to avoid similar events in the future. Some of these reports give a picture of the deformed school bus after the accident. They also provide an explanation of the accident scene to aid visualization of the event. The investigation of the accident is conducted by tracking the driving records and other information about the school bus driver and the driver of the vehicle that was involved in the accident. The velocities of the vehicles before the accident are deduced from the tire patch and deformation of the vehicles after the accident. Reports are checked for the year in which these accidents occurred, to make sure that the contents and the model of the bus involved in the accident were not very old to make a comparison with the results obtained from these simulations. 
These reports are the only data found for the comparison of the crashworthiness simulation results for this project. Though the National Highway Traffic Safety Administration (NHTSA) conducts several tests on school buses by crashing them, none of them were looked in the perspective of the crashworthiness of the whole bus in entirety.

\subsubsection{VAN BASED TEST}

Van based school buses are classified as Type A school buses where the gross vehicle weight is less than $4545 \mathrm{~kg}(10,000 \mathrm{lbs})$ and designed for carrying ten or more passengers. The school bus simulated in this project is called the Type $\mathrm{C}$ school bus or the conventional school bus whose gross vehicle weight is more than $4545 \mathrm{~kg}(10,000$ lbs) and designed for carrying more than ten passengers. All of the engine and engine components are in front of the windshield, and the entrance door is behind the front wheels. A crashworthiness test was conducted on a van based school bus (Type A) by the National Transportation Safety Board (NTSB); however the bus model was not the same as the one simulated in this project (Type C). Tests are often conducted to check the brakes in the vehicle, damage to the fuel tank in the vehicle, hand rail designs, safety belt use and joint strength. The Federal Motor Vehicle Safety Standards ${ }^{11}$ (FMVSS) are defined for the performance of various systems of the bus like the hydraulic brake system, air brake system, safety belt assemblies, window retention and release, bus body joint strength, fuel system integrity, etc. A list of Federal Motor Vehicle Safety Standards is listed in Appendix A. 


\subsubsection{Type A Pillar Crash}

A highway accident investigation reports the loss of control and collision of a small school bus (Type A) with a guard rail and sign pillar on November $11,1985^{27}$. The activities of the school bus driver were investigated first with a detailed investigation on the background of the driver. The vehicle was investigated for any damage, and the highway weather and road conditions are also take into account. The pitman arm that controls the vehicle steering was subjected to a metallurgical tests, which did not show any preexistent failure of the component. The bus body was forced off of the chassis of the bus, which had positive safety results since crash forces transmitted to the school bus body were reduced. It was concluded that the school bus driver was driving the bus under the influence of alcohol, which was the cause for the accident. This report does not look in to the crashworthiness perspective of the accident and it does not give any picture of the final deformed shape of the bus.

\subsubsection{CRashworthiness Study OF TyPE A BUSES}

The National Transportation Safety Board (NTSB) performed a safety study on the crashworthiness of small post standard school buses ${ }^{28}$. The crashworthiness study was conducted on a Type A school bus that weighed less than $4545 \mathrm{~kg}(10,000 \mathrm{lbs})$ and designed to carry more than ten passengers. A small post standard school bus was crashed and the damage to the exterior and the interior of the bus was documented and analyzed, in relationship to each passenger's seating position. For each occupant, the investigators attempted to determine whether a restraint system was used, whether it was used correctly, the probable cause of injury, and the nature and severity of the injury sustained. Because of the differences in the size, mass and exterior features, the findings 
from a small school bus cannot be extrapolated to a large school bus. It was concluded that the small school buses involved in the accidents investigated provided good crash protection.

\subsubsection{TYPE A SIDE IMPACT}

A highway accident investigation reports the collision of a small school bus (Type A) with a tractor-semi trailer on November $10,1993^{29}$. The investigation reported the activities of the school bus driver before the accident and the condition of the bus; before the event a 72 hour history of both the drivers was investigated along with the work experience and the driving record of the drivers. The school bus was damaged along the doorway side of the body from a side impact. The contact deformation began at 22 inches from the rear corner of the doorway and extended up to 96 inches, approximately equal to the width of the tractor trailer. The maximum intrusion into the impact side of the bus was 29 inches. An analysis of the sequence of events was made, and it was concluded that the right front vertical support structure obscured the bus driver's view for 5 to 7 seconds before the collision and this was the cause of the accident.

\subsubsection{TYPE A CRASH WITH A LOCOMOTIVE}

A highway accident investigation reports the collision of a locomotive with a school bus (Type A) at a railroad grade crossing on October $25,1995^{30}$. The investigation reported the activities of the school bus driver before the accident and the condition of the school bus before the accident. An investigation was also made of the train crew and the mechanical conditions of the train. Analysis was made of the school bus driver's performance, road design, rail road-highway sign interaction, and other factors that might have caused the accident. It was concluded that the bus driver did not heed to the 
warnings that a train was approaching the crossing and hence caused the accident. Also the training provided by the school district to the bus driver during the emergency was ineffective.

\subsection{Prior BUS/Truck Numerical Models}

Trucks and buses were not modeled completely to study the crashworthiness of the vehicle structure. In some cases, only the occupant area was modeled with simple beam elements that could help to study the structural integrity of the bus. The National Highway Traffic Safety Administration (NHTSA) reports that 1,478 people have died in school bus related crashes since 1985, out of which 11 percent were occupants of school buses $^{11}$. However NHTSA, has conducted tests and setup standards for the various components of the bus like the fuel tank, brake system, safety belt assemblies, and bus body joint strength.

Monasa $^{1}$ of the Civil Engineering department of Michigan Technological University and McGuire of the Structural Engineering department of the Cornell University in 1986 modeled the passenger compartment of a small bus. This model was created to study the collapse performance and the structural integrity of the model, which could determine the ultimate failure load and the deflection characteristics under specified loading conditions. The passenger compartment of the bus was mounted on and supported by a separate chassis. Interactive computer graphics were used to preprocess and post process the model. The data was input through a graphic user interface and not through digital means. A preprocessor called FRAME3D was used and solved in a software called ANALYSE. A roll over load was applied at a node and the deformed 
shape was determined. A program called SLD3D, which could perform a full range of analysis from linear elastic to non-linear analysis, was used to solve the model. The program was able to account for both types of non-linearity (i.e) geometric non-linearity and material non-linearity. The program incorporates the material non-linearity by attaching plastic hinges at the ends of the elements. Including a flexural member geometrical stiffness matrix into the direct stiffness method incorporates geometric nonlinearity. The results showed a parellelogramming mode of deformation under the roll over load and a passenger safety compartment in the structure was determined.

Mahesh $^{7}$, Subash and Yan of Concordia University worked on the crashworthiness enhancement in a car-truck collision by providing an energy dissipative under-ride guard. The energy dissipative under-ride guard helps to absorb energy when a lightweight vehicle such as a car is involved in a collision with a heavy truck. Since the kinetic energy of the heavy vehicle is very high compared to that of the car, the possibility of heavy damage to the occupants in the smaller vehicle is high. So, energy dissipative under-ride guards with hydraulic dampers attached to the rear side of the under-ride guards provide excellent energy absorbing capabilities. By using the basic equations of motion, and equating the moment of the momentum after the impact in the under-ride guard, the velocity of the car at the point of impact was determined. Also the magnitude of the acceleration of the car mass and the dissipated energy under direct impact is determined. Also two different kinds of conventional under-ride guards were modeled in LS-DYNA3D and the results of the conventional guard were compared to those of the proposed under-ride guard. From the results, the normalized energy absorbed by the 
proposed under-ride was found to be nearly twice as much as that absorbed by the conventional under-ride guard.

Saunders ${ }^{14}$ of West Virginia University studied the feasibility of an articulated seat as a means to restrain the children without seat belts in the event of collision or sudden stop. Upon collision the seat moves with the child in such a way that it assists in restraining the child and thereby reducing the possibility of a collision between the child and the back of the adjacent seat. A computer-aided analysis of an articulated school bus was performed. Also a standard seat was compared with the articulated seat and differences in acceleration, velocity, and displacement were determined. I-DEAS Master Series was the preprocessor used and the LS-DYNA3D was the solver used to simulate the model. This study showed that the articulated seat mechanism has an important effect on the child's dynamic behavior. A limitation of the analysis was the lack of information on the boundary conditions used for the analysis which was the genesis of this work.

\subsection{Modeling Approaches to Vehicle Crashworthiness Simulation}

As with all numerical models, the size and description of the finite element model is determined by the power of the computers. In the initial models of the 1960's and 1970's, a detailed geometric description was avoided to reduce the time taken for solving the model. Most of the components were modeled as lumps of masses and were connected using springs to reduce the number of elements and hence the computation times. As computing power increased, the finite element models became more refined with greater geometric details. With small element sizes and a number of elements, the deformation 
results have improved. Models with around 500,000 elements are now solved easily by super computers.

$\mathrm{Shah}^{2}$, Reid, Lesh and Cheva of General Motors Corp. studied the mesh sensitivity for numerical modeling of a vehicle for crashworthiness. The numerical model of a vehicle for crashworthiness can be done in two ways, lumped parameter (LP) modeling and finite element (FE) modeling. They conducted experimental tests on the vehicle for LP and FE techniques to evaluate the compatibility and the interrelationship of the two methods. The LP modeling uses a 1-D spring mass system to represent the vehicle to be crashed. The model could be built very easily, but most of the information is based on experimental tests of components and hence it needs good experience to work on this area. With the advent of high speed computers, there has been a significant amount of research conducted in the area of finite element technology and it has been used in the automotive industry since 1980's. With the use of sophisticated preprocessors, a complete detailed model of a vehicle can be made and studied for crashworthiness. But the time taken to model a vehicle and the cost of computation makes this method more cumbersome than the previous method.

There are three different factors to be considered while creating the lumped mass model of the vehicle. The first, called the topology, refers to the number of springs and masses in the model. The topology can be refined and simplified by eliminating insignificant masses and load paths. Also, the symmetry of the model can reduce the number of springs and masses in the system. The second factor is the mass distribution in the model; larger masses decrease the accuracy of the results with decreased output information. The third factor is the force deflection curve of the mode, which shows the 
energy absorbing capability of the model. The force deflection properties can be obtained by hand calculations, past model data, results from FE models, and from physical tests. Apart from these three factors the dynamic factors such as strain rate sensitivity, wave propagation, and inertial effects should also be considered. When all three factors are optimized, the model behaves to give a more accurate result.

Explicit non-linear finite element method modeling involves four major steps: identifying necessary components to be modeled, selecting proper material model that represent them, connecting the various components through coupled degrees of freedom, and solving the system to obtain the necessary output. The authors also conducted a mesh sensitivity study by modeling a rectangular tube using three different meshing arrangements. Model A had uniform 10x10 mm mesh, whereas models B and C had both 10x10 and 10x15 mm meshes arranged in different patterns. Mild steel properties were given to the tube. The velocity and the boundary conditions were the same for all the models; the only difference was the mesh size.

The results are shown in Figure $2.1^{2}$, which indicates that the mesh size affects the deformation pattern or the crush kinematics to a considerable extent. In order to obtain a realistic deformation pattern, the mesh size should be chosen carefully. 


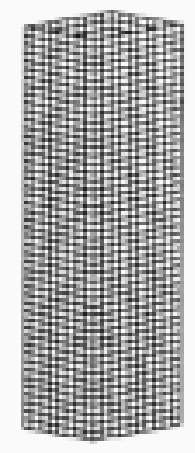

\section{Model A}
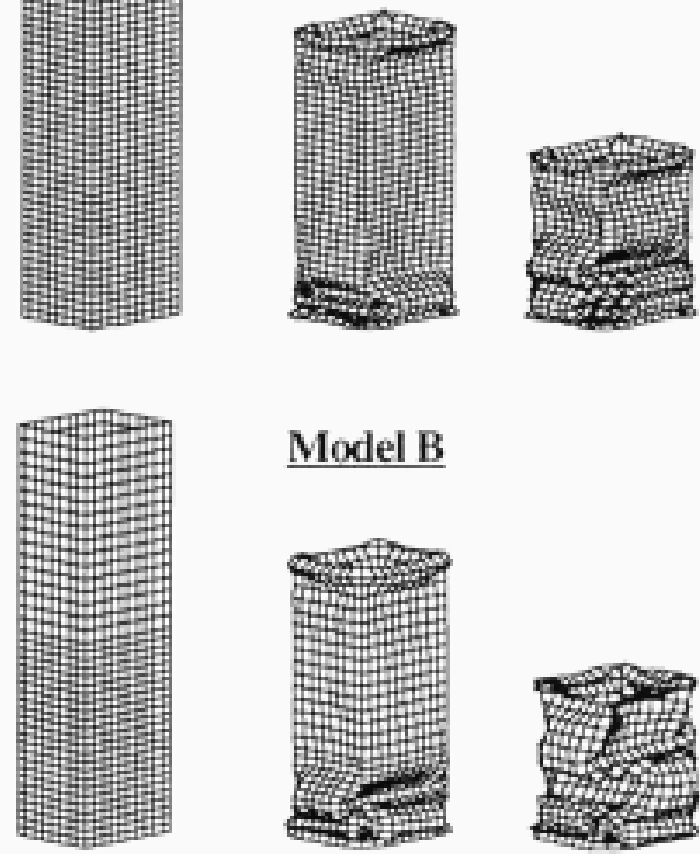

\section{Model B}
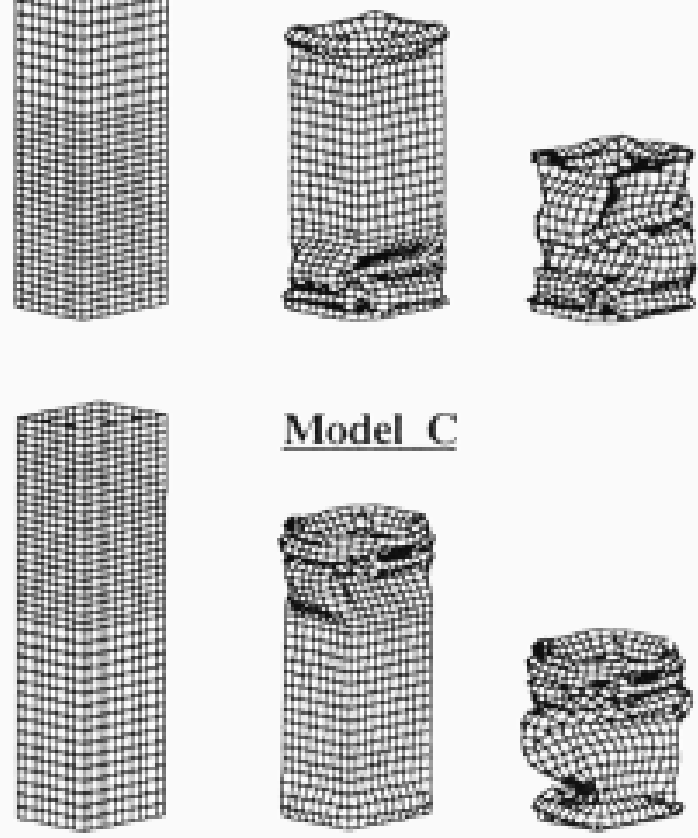

\section{Model C}
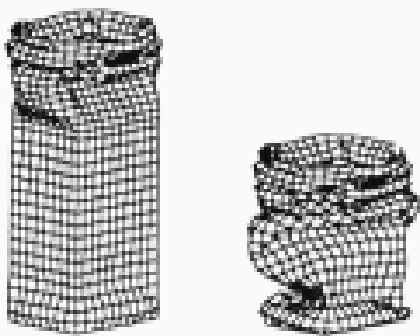

Fig 2.1 Influence of mesh density on displacement.

Onusic $^{3}$, Campos and dos Santos of Mercedes-Benz Brazil worked on the simplified hand calculations of the impact forces during vehicle collisions. There are many computer programs trying to simulate crash tests, but in order to get an idea about the magnitude of the impact forces that are to be obtained from the simulation, a hand calculation can be done using the physical concepts, mechanics, and simplified 
approximations. These collision events are similar to a projectile impact on a rigid wall. A constant deceleration force is considered and it is assumed that the deceleration force varies linearly with the time. Cases were considered assuming the vehicle to be extremely rigid and have a highly inelastic soft body. The effect of the elastic parts such as the power train was also considered.

The impact is assumed to be equivalent to the deceleration of the vehicle with the mass at the center of gravity of the vehicle. The basic equations of dynamics give

$$
\mathrm{V}^{2}=\mathrm{V}_{0}^{2}-2 \mathrm{ax}
$$

and

$$
\mathrm{V}=\mathrm{V}_{0}-\text { at }
$$

Giving the end conditions $V=0$, and assuming the vehicle travels for a distance $\frac{\mathrm{L}}{3}$,

where $\mathrm{L}$ is the length of the body, then

$$
\mathrm{a}=\frac{3 \mathrm{~V}_{0}^{2}}{2 \mathrm{~L}} .
$$

For, $\mathrm{F}=\mathrm{M}$ a, then

$$
\mathrm{F}=\frac{3}{2} \mathrm{M} \frac{\mathrm{V}_{0}^{2}}{\mathrm{~L}}
$$




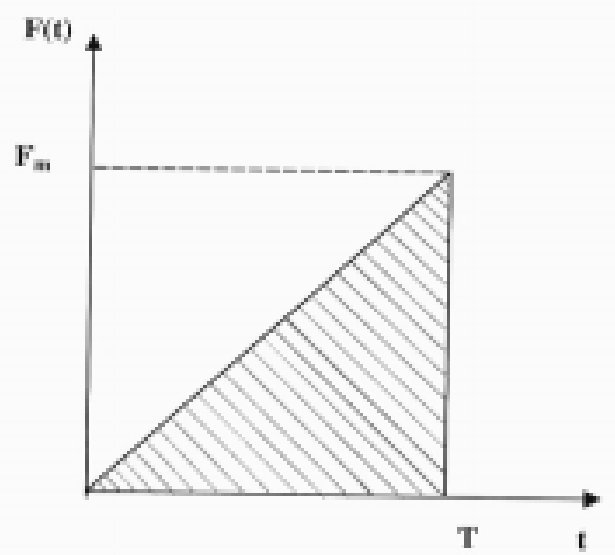

Fig 2.2 Graph showing the deceleration force varying linearly with time.

Cases were considered assuming that the deceleration force varies linearly with time: it is null at the beginning and reaches a maximum at the end of the impact as shown in Figure 2.2 $2^{3}$.

The acceleration is also linear since the force is linear and is defined as

$$
a=-\alpha t
$$

At the end of collision,

$$
\frac{\mathrm{F}_{\mathrm{m}}}{\mathrm{M}}=-\alpha \mathrm{t}=-\alpha \mathrm{T}
$$

or

$$
\alpha=\frac{\mathrm{F}_{\mathrm{m}}}{\mathrm{M} \mathrm{T}}
$$


Integrating the acceleration, the velocity is determined and the constant of integration is $\mathrm{V}_{\mathrm{o}}$ :

$$
\mathrm{V}=\mathrm{V}_{\mathrm{o}}-\alpha \frac{\mathrm{t}^{2}}{2}
$$

Integrating the velocity, the displacement is

$$
x=V_{o} t-\alpha \frac{t^{3}}{6}
$$

When the center of gravity reaches a zero velocity, $\mathrm{V}=0$; substituting this back into equation (2.9) yields

$$
x=-\alpha \frac{t^{3}}{6}
$$

The time taken for impact is given as

$$
\mathrm{T}^{2}=2 \frac{\mathrm{V}_{\mathrm{o}}}{\alpha}
$$

The impulse of the force can be calculated from the area under the curve in Figure 2.2

Calculations were also made considering the vehicle as an extremely rigid body and as an extremely soft body. When the rigid body hits a rigid wall, a shock wave will be transmitted through its body travelling the length of the body and reflecting back and forth. The velocity of the shock wave will be equal to the velocity of the sound in the material. The equation for the impulse force is given by 


$$
\mathrm{F}=\gamma \mathrm{M} \frac{\mathrm{V}_{\mathrm{o}}}{\mathrm{L}}
$$

where

$$
\gamma=\frac{\mathrm{C}}{\mathrm{V}_{\mathrm{o}}}
$$

When the vehicle is considered as an extremely soft body, it can be imagined as a bag of fluid. When the vehicle hits the wall, the rear portion of the body doesn't experience any load as per this assumption. The mass progressively touches the wall and spreads on its surface. The shock is perfectly inelastic. The impulse force is given by

$$
\mathrm{F}=\mathrm{M} \frac{\mathrm{V}_{\mathrm{o}}^{2}}{\mathrm{~L}}
$$

The results were applied to a passenger car and a bus, and the results were underestimated in relation to real data. Also it was observed from the elastic and inelastic models that the reduction of wave shock preserves the rear parts of the vehicle.

Ingarashi $^{4}$ of Suzuki Motor Co. performed a body structural analysis of a 1989 Suzuki Sidekick at various stages of development and design. This paper illustrates that the application of the structural analysis for the body structure along with the experiment is beneficial if applied at the earlier stages of design. There are three stages in the development of car body structure. The first stage is to develop a body structure that satisfies the basic requirements of a car body such as structural rigidity, weight, natural frequency, and crashworthiness. The second stage studies these factors in more detail and evaluates these factors; and this stage results in detailed design drawings of the body 
structure. In the third stage, the results obtained are compared with the experimental results from a prototype.

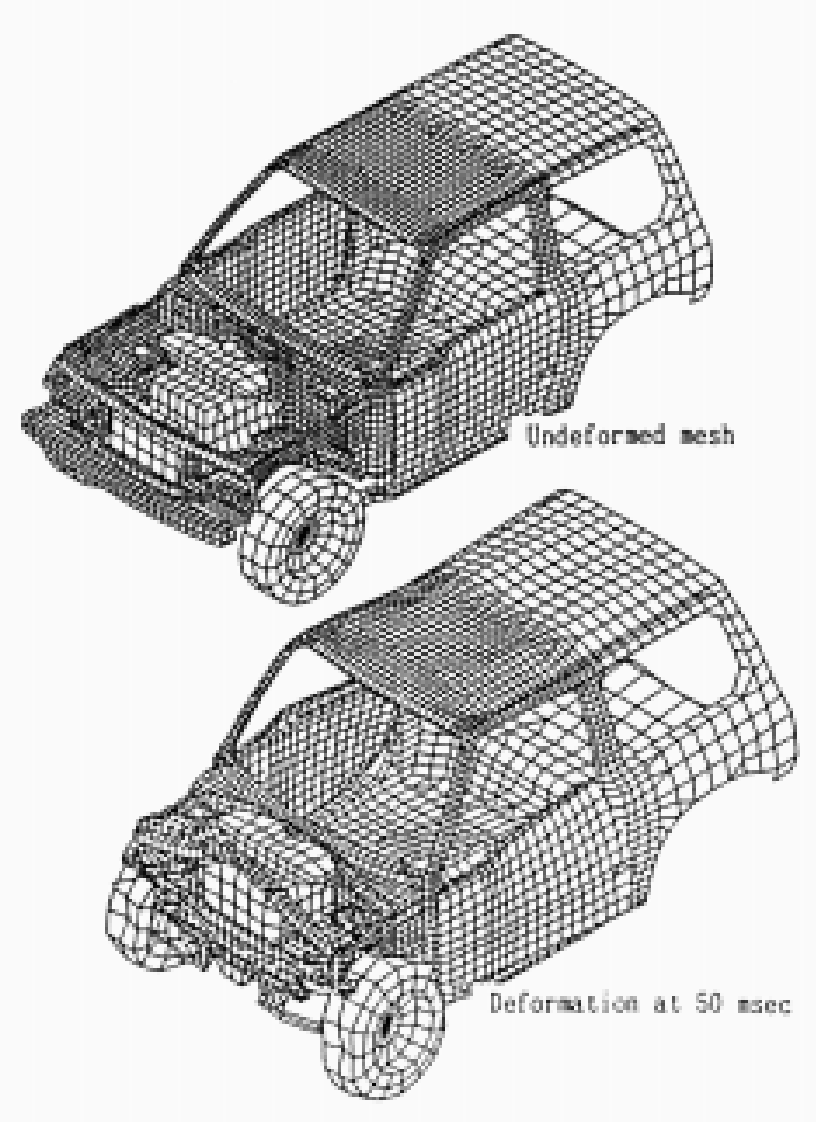

Fig 2.3 Deformation of the Suzuki Sidekick finite element model.

In the first stage of the design development, the model was simulated for crashworthiness. For a frontal barrier crash, it is desirable to minimize the damage in the occupant area and maximize the energy absorption by the components in front of the firewall. The frame should absorb the kinetic energy of the vehicle when the vehicle impacts the barrier. Also certain factors like lightweight and vibration control should also be considered when the frame is designed. A beading was placed in the central portion of the front frame to buckle itself and absorb the energy. The impact energy (E) absorbed 
can be expressed as a product of the buckling force $(\mathrm{F})$ and the amount of deformation (D). The buckling force or the amount of deformation is equal to a function $\mathrm{f}$ (location of the beading, gauge thickness, cross section of the frame, shape of the frame). Also a detailed analysis of front-end components like the bumper was also carried out. The frame designed for the vehicle was a 3-partitioned frame unlike a conventional one, and hence the frame had to be tested for its strength and durability. Experiments were carried out on a similar vehicle and the loads coming on the frame were calculated experimentally. From then, these loads were applied to the 3-partitioned frame under development and a finite element analysis was carried out.

A design change in the later stage becomes difficult if the cross sectional details are not studied in detail in the earlier stages of design. So, at an earlier stage of design, the basic body structure was modeled by using bar elements to optimize the body structure as much as possible. The advantages of this simple model are the ease of calculation, ease of incorporation of changes in design, and reduced time. A skeleton model of a similar vehicle was created and analyzed and compared with the experimental results to make sure the design process is moving in the right direction. Once the skeletal model was checked for the design durability and vibration, a detailed fine grid mesh of the entire vehicle body was created. Then using the fine grid mesh, the model was checked for rigidity and vibration to make sure that the structure will not exhibit a resonance with the disturbance and the exciting force exerted on the body. A crashworthiness simulation was carried out using the fine grid mesh of the vehicle body for frontal and rear barriers. Only half of the model was analyzed owing to the symmetry of the model. Rigid elements were used in places where there are no deformations. The model was given an initial 
velocity of $35 \mathrm{mph}$ and the calculation was terminated after $100 \mathrm{~ms}$. The experimentally determined body deceleration and body velocity were compared with the simulation results and a good correlation was found.

Lugt $^{5}$, Chen and Deshpande of General Motors Corp worked on the numerical simulation of a passenger car frontal crash at $48 \mathrm{~km} / \mathrm{hr}(30 \mathrm{mph})$. The model was solved in PAM-CRASH, which is an explicit finite element code. In crashworthiness, an effective design is the one which effectively absorbs the energy required to protect the occupants and meets all other requirements. The explicit method can take full advantage of the modern day super computers rather than the implicit method. PAM-CRASH is an explicit finite element code, which directly yields the nodal accelerations solving the nonlinear equations of motion. This produces a diagonal lumped mass matrix, which can be solved easily by a computer. The velocities and displacements are obtained from the nodal accelerations by the central difference method. The size of the integration time step is a limitation to the explicit method. For the solution to remain stable, the time step must be smaller than the time it takes for the sound to travel through the smallest element in the mesh. If this condition is not met, the solution may not converge to the exact solution and might diverge to a different one. Hence the explicit method is extremely useful for crash events.

The model of the vehicle was created with a closer mesh density at the front end where there is large deformation and sparser mesh density at the middle portion of the vehicle where the deformation would be much less. An increase in the mesh density at the front end increases the chances of capturing even the smallest buckling in he vehicle. However the integration time step is controlled by the smallest element in the mesh and 
hence the cost of computing would also be high. Only the front portion of the vehicle was modeled and the rear portion was given as a concentrated mass at the appropriate places to produce the correct center of gravity for the vehicle. The model was solved for 48 $\mathrm{km} / \mathrm{hr}(30 \mathrm{mph})$ and the deformation shapes were obtained. An experimental test was also conducted; the final deformation shape of the vehicle was compared and a good agreement between the results was determined.

Belytschko $^{6}$ of the Civil and Mechanical Engineering Department at Northwestern University presented the evolution of computers for crashworthiness and the computational methods available. The improvement in computational methods shows up as more effective time integration procedures and more efficient elements. In the mid 1970's the time required for solving a 500 DOF model of a Plymouth car took up to twenty hours of running time for the solver in the largest mainframe at that time, costing up to $\$ 1000$ an hour, though the results of the simulation were comparable with the test results. The next principal development in the area of crashworthiness was the 4-node quadrilateral thin shell element, which required only one quadrature per element. This helped to decrease the time for computation by tenfold. At the same time, a dynamic analysis code called LS-DYNA3D was developed by Lawrence Livermore National Laboratory, which used explicit time integration and was completely vectorized to take advantage of the Cray computer architecture. The only disadvantage with the explicit method is that a small time step has to be used in order for the solution to be stable. The time step should be less than the time required for the elastic wave to travel the size of the smallest element in the model. So if the model has even one small element, the time required for the entire solution is increased. This was overcome by the multi time step 
integration. In this method, the elements are divided into groups and a different time step is used for each group, so that if only one group has small elements, they alone are integrated with a smaller time step. One of the major improvements in the area of mesh creation is the adaptive meshing technique. By this method, the mesh is refined during the course of the computation in order to achieve the desired accuracy.

Piatak $^{8}$ of ASC Inc., Sheh of Cray Research Inc; and Young and Chen of Optimal CAE, Inc worked on a convertible crashworthiness design using non-linear finite element methods. The frontal and rear impacts of the convertible car were studied using the dynamic non linear finite element code LS-DYNA3D, and the results were verified with the experimental crash. Most of the vehicles were not designed with the convertible option in mind, and as a result of the conversion process the vehicle loses its structural stiffness to a large extent. Convertibles are modified two door coupes with the roof and the pillar being replaced by a retractable top. Since the pillars are removed, the load path through the roof structure is affected, and most of the impact is transferred through the door structure and the lower load path such as frame and rocker. Without proper reinforcement, during a crash event there might be excessive damage to the doors and floor and there are chances of impact entering the occupant area.

LS-DYNA3D was used for designing the front structure reinforcement package design and deck lid crashworthiness. When the actual vehicle was crashed at an impact velocity of $56 \mathrm{~km} / \mathrm{hr}(35 \mathrm{mph})$, the test results showed significant deformation of the front structure leading to inadequate occupant kinematics. The test vehicle was stripped down to study all the deformations in detail. The poor performance of the vehicle was attributed to the lack of roof structure. Various alternatives were suggested to overcome 
the problem, and it would be very costly and time consuming to evaluate all the suggestions by experiments. To compare the effectiveness of the various reinforcement scenarios, only the front half of the body was modeled. The model had approximately 28000 nodes, and 27000 shell elements, and 1200 spot welds. A symmetric boundary condition was defined and a rigid barrier was defined to move at a constant velocity of 56 $\mathrm{km} / \mathrm{hr}$ (35 mph) impacting the front end of the vehicle. One of the suggestions proposed a tubular brace between the firewall and front barrier to stabilize the shock tower deformation. This proposal, when modeled in LS-DYNA3D and analyzed, gave the least amount of header drop and pillar rotation. When this model was tested experimentally, at $56 \mathrm{~km} / \mathrm{hr}(35 \mathrm{mph})$ against frontal barrier, the pillar experienced much less deformation. In addition both the driver and the passenger doors opened easily after the crash, which helps the passengers to exit.

The deck lid is a new design that covers the trunk opening when the retractable top sits on the trunk. It is hinged at the rear and latched at the front. In the previous $35 \mathrm{mph}$ rear impact test, the deck lid was not sufficiently crushed, transmitting a large amount of forces through the front mounting brackets. The requirement is that the deck lid should sit on the trunk opening after the rear impact. Various proposals were made and a finite element model of the deck lid was built consisting of 5500 shell elements and constrained at three mounting bracket locations. One of the proposals which suggested with two crush initiators near two front mounting plates and one at the center with thinner and outer panels behaved better with lesser peak force in crush. Experiments were conducted and the results compared well with an experimental peak force of $20.5 \mathrm{kN}$, giving a simulated force of $19.8 \mathrm{kN}$. 
Garrot $^{19}$ and Flick of NHTSA and Mazzae of Transportation Research Center, Inc. worked on the comparison of electronics based object detection systems for heavy trucks. The object detection systems can be classified into two groups: rear object detection systems and side object detection systems. The rearward sensing systems are intended to help drivers when they are backing their vehicles at very low speeds in order to avoid crashes with parked vehicles or pedestrians or other fixed objects. Comparison was made among six commercially available systems. The side object detection system is used as a supplement to the outside rearview mirror systems and as a means for detecting adjacent vehicles when making a lane change or merging maneuvers. Changing lanes towards the right is a difficult task for drivers in dense traffic situations. Four such systems were evaluated and compared. Data from National Accident Sampling System shows that in 1991 an estimated 190,000 crashes involved combination unit trucks resulting in 4,849 fatalities, of which 3764 were occupants of other vehicles involved in the crashes and 659 were occupants of the heavy trucks.

From the study, conclusions were made about the rear object detection systems. Compared to vehicles that are not equipped with these systems, the rear object detection systems may have the potential to improve safety and reduce accidents while backing. A rear mounted video camera with microphone helps the driver to see and hear what is behind the vehicle. The fields of view of the cameras were evaluated and found to be adequate for most backing situations. The information obtained by the camera is transmitted to the driver in an easily-understand form. Conclusions were made about the side object detection systems and these systems may provide benefits to drivers by greatly increasing the visibility and awareness of the drivers as to what is happening on 
the lane to the right. Especially in areas of dense traffic, these detection systems may help the driver to make quick decisions about whether or not it is safe to change lanes. Also, improvements in these systems are necessary to receive significant benefits.

Rechnitzer and Murray ${ }^{20}$ of Monash University and Scott of VICROADS Road Safety Inc. worked on a detailed investigation of the injuries to car occupants in rear end impacts with heavy vehicles. Also, a demonstration of an effective lightweight inexpensive barrier on the rear of the vehicle was made that reduced injuries during an underrun.

The rear underrun is described as the front of a car or any other small vehicle that follows the truck, sliding under and colliding with the rear end of a truck or trailer. This underride occurs because the rear end of the truck is high off the ground and there is nothing to resist the vehicle that strikes the back of the truck in case of an emergency braking. This type of crash results in a severe injury to the occupants of the smaller vehicle that hits the truck and in most of the cases the rear end of the truck crashes through the wind screen and strikes the front passenger compartment. In the United States, 350 people were killed in underride crashes in 1978. The possible injuries to the occupants of the car were multiple lacerations on the forehead and face and fractures of the skull and frontal bones. Also, occupant protection systems like the airbag and the seat belt are quite ineffective and are more likely to result in direct occupant impact with the truck structure resulting in severe or fatal head and chest injuries.

To prevent injuries to occupants of cars colliding with the rears of truck; the first prerequisite is to prevent excessive underrun. The second consideration would be to 
reduce occupant compartment injuries and to reduce the deceleration of the passenger. The first prerequisite can be achieved by attaching a barrier of sufficient strength and appropriate height to the rear of the truck. This barrier should be able to absorb energy effectively and resist the impact load from the car. In this case, the occupant survivability becomes a factor of the occupant protection systems like the airbag and the seat belt and the crashworthiness characteristics of the car. If the barrier is deformable and capable of absorbing large amounts of energy, then the risk of injuries would be reduced further. Also the barrier should work effectively if the impact is not only central, but also for side impacts, in which case the load is not distributed over the full length, but resisted only by a part of the barrier structure.

It was concluded that the truck without a rear end rigid barrier would cause more injuries and fatalities to the car that crashes the rear end than a truck that is fitted with a light weight barrier that could absorb energy and prevent a car underrun. Also it was concluded that the loads imposed on barriers during the test exceeded the loads stated as design loads in the proposed United States rules.

Shiosaka $^{21}$ of Japan automobile research institute investigated the evacuation readiness of the bus crews and passengers and studied the effect of a new type of exit for the passengers. The new type of exit was developed based on the results of current bus exit performance tests. Also, tests were conducted to measure the time required to evacuate using the improved emergency exit. Group evacuation tests through windows were carried out to compare the time required to exit in the regular and the new emergency exits. The new emergency exit aimed at improving the safety for passengers with diminished physical functions and for aged persons while making an emergency 
exit. The exit was mounted on the rear mock up and one end of the door is supported and the other end is free to fall on the ground. In case of an emergency, the door swings open about its support and rests on the ground giving a support to the escape slide that inflates and allows the passengers to slide and come out. The new exit is shown in Figure $2.4^{21}$.

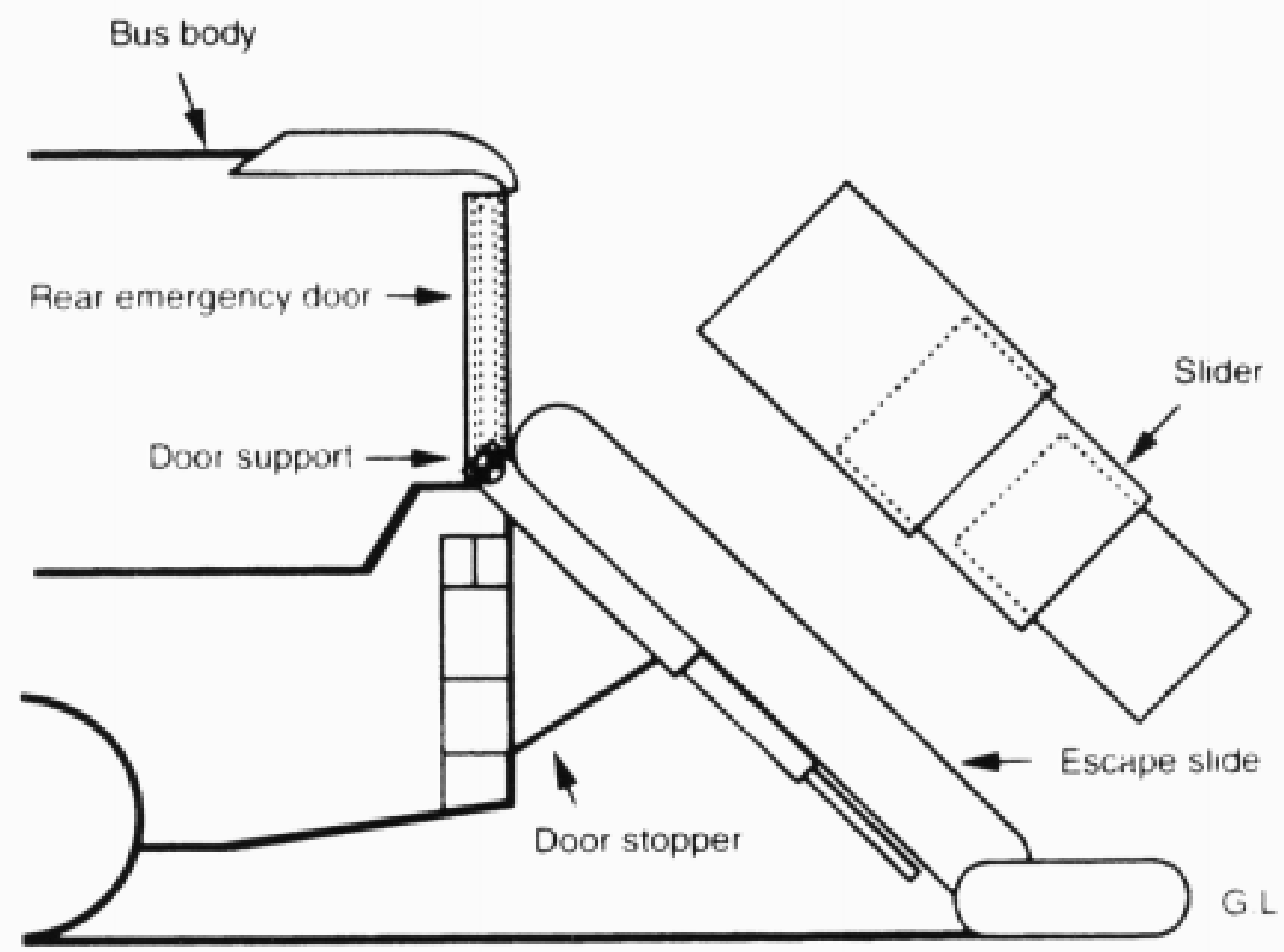

Fig 2.4 New emergency exit.

The new type of exit helps to evacuate aged people who think it is dangerous to jump outside the window for a emergency exit. When tested, the maximum time required only three minutes to evacuate the entire crew. 
Kaufman and Gaines ${ }^{22}$ of Ford motor company, Kundrick of Tower Automotive Inc., and Sheng-Dong Liu of National Steel Corporation worked on the integration of the frame side rail forming analysis into the finite element crash model. For vehicles that have the body on the frame, the chassis truck frame absorbs about $70 \%$ of the total kinetic energy created during the frontal impact. Traditional crash analysis utilizes the standardized material properties for the finite element model. When these properties are used, the steel does not reflect any strain hardening effects that occur during the forming process. The properties from the forming analysis like the material thin out, yield strength, and tensile strength were input into the crash finite element model of the vehicle. These properties modified the frame deceleration pulse and buckling mode characteristics in the crash output.

The frame structure is designed to meet a target stiffness that absorbs kinetic energy from frontal impact. Despite the efforts of modeling the frame carefully, the model might not be a complete representation of the vehicle because the raw steel properties assumed for the frame are not the same as the formed steel properties. When the raw steel properties are used for the frame in the finite element model, the non linear performance analysis does not take into account the change in material properties during the forming process. The flat stock undergoes strain hardening and changes in the yield strength during the stamping process. A new process was proposed that could use the final properties of steel in the finite element model.

In the new method of analysis, the starting point remains the same as the existing process; raw steel material properties are provided to construct a finite element model. A forming analysis is performed first before any crash analysis is performed. The forming 
analysis software gives the final shape of the frame, which can be completed in a half day. Using the same frame model for the forming analysis and the performance analysis allows for easier implementation of the material properties and it could also lead to the automation of the forming results into the starting point of the performance analysis. Upon completion of the forming analysis, the material properties are updated in the finite element model and the model is transferred to undergo a crash analysis. The strain hardening in the model, the greater the effect on the yield strength and tensile strength. The proposed method was implemented on a vehicle CAE program.

The crash finite element model was created and transferred to the steel supplier to complete the forming analysis. After the forming analysis, a crash analysis was performed and the results were determined. A standard crash analysis was performed using the raw steel data and the results were found to differ. The new method agreed closer to the base line.

Tanner ${ }^{23}$, Chen, Wiechel, Brown of S.E.A Inc., and Guenther of Ohio State University studied the effect of low speed rear impacts on the vehicle and the occupants. A more common type of rear impact is the one in which the bumper of the striking vehicle rides over or under the rear bumper of the struck vehicle. One example of this case is the car running under the rear end of a truck. These types of impact cause more injuries to occupants than the bumper to bumper impacts at identical speeds. Several kinds of such impacts were tested with Hybrid III dummies or human volunteers.

Three separate vehicle configurations were utilized to perform the crash study. In the first test series, the striking vehicle was a Kenworth conventional tractor and the 
struck vehicle was a 1981 Ford Mustang. The front bumper of the Kenworth is mounted directly on the frame of the vehicle and the rear bumper of the mustang consisted of a bar mounted to energy absorbers. In the second test series the striking vehicle was a 1982 Plymouth Horizon and the struck vehicle was a 1986 Chevrolet S-10 Blazer 4X2. The front bumper of the Horizon consisted of a plated aluminum extrusion that mounts on to energy absorbers that attach to the vehicle frame. The rear bumper of the Blazer is chrome plated steel attached to the vehicle frame through mounting brackets. In the third test series, the striking vehicle was a Mack conventional tractor and the struck vehicle was a 1986 Toyota Camry. The front bumper of the Mack was the same as the Kenworth in the first series and the rear bumper of the Camry was constructed from a rubber cover over an energy absorbing structure that mounts on the frame.

Results were obtained for the three series of vehicles. In the first series, the most important result was that there was no capacity for energy absorption on the front surface of the Kenworth tractor. Though the rear of the Mustang had an energy absorber, the speed of the impact was insufficient to cause any significant energy absorption. In the second test series, the energy absorbers of the Horizon worked as they were designed to and stroked completely. At a velocity of $12 \mathrm{~km} / \mathrm{hr}(7.5 \mathrm{mph})$, the energy absorption capability of the Horizon was overcome and the bumper structure collapsed and rode under the Blazer bumper which penetrated into the grill and front structure of the Horizon.

This study gave an opportunity to examine the effect of override and underride on vehicle impact response at low speeds. Since the impact is over the bumper or under the bumper, the minimum velocity to cause a damage to the vehicle would decrease. But 
from the results of the tests conducted, it did not seem to be true. Also, at higher speeds where the damage is expected to be more, the involvement of the softer vehicle structures in a crash would result in reduced forces and impact.

Smith and Ravipati ${ }^{13}$ of West Virginia University worked on the crashworthiness of a passenger car model subjected to full frontal, offset frontal and pole collision events. Only front end of the vehicle was modeled to efficiently simulate the front end model for the majority of collision events. The complete model consisted of 18208 nodes, 16463 shell elements, 1243 solid elements, and 8 beam elements. The full frontal collision event results were compared with the DOT supplied test data for validation. An hour glassing problem was encountered when the model was solved for frontal impact. When the kinetic energy, internal energy and the total energy was plotted against time, there was a sharp increase in the total energy at around $30 \mathrm{~ms}$. When the model was examined at this point, it was found that the engine came into contact with the core support at this time, and the average element length in the engine was much larger than the one in the core support. The element length was reduced to a finer mesh and the solution was run again. This result showed no abrupt change in the energies. One more way of avoiding this problem would be to do eight point integration, which may cost more than reducing the element length. So care should be taken that the ratio of the element lengths of the contacting faces is not large. Also, the experimental and the simulated energy absorption during the full frontal collision event matched well and the results were verified. 


\subsection{Software Packages for Vehicle Crashworthiness Simulation}

I-DEAS Master Series ${ }^{9}$ is one software package in the area of mechanical computer aided engineering (MCAE) that could integrate design and analysis. This software provides a series of applications starting from solid modeling to finite element analysis, design optimization and testing in the area of $\mathrm{CAD}$, and a series of applications from solid modeling to master assembly and manufacturing in the area of CAM. These applications are fully integrated so that one can transfer the model from one module to another. The I-DEAS Master Series 4 has some advanced features like sketch in path, sketch in place, and several surfacing options that help to create a solid model easily.

The I-DEAS Master Series has good team engineering capabilities; a model can be checked into the library of the software, and anyone working on the same group can use the model for reference and pick out a copy of the model from the library. Also, I-DEAS is compatible with most other finite element software and it can read a wide range of geometry translators like IGES, DXF, and other formats. Owing to these excellent capabilities, this software was chosen for creating the geometric model of the bus.

The I-DEAS Master Series was used as a preprocessor for the finite element model developed. This software can write an ASCII file that contains the nodal and elemental data for the model along with the material properties and physical properties. Some standard translators that could write out a LS-DYNA3D input file can read this universal

file. One such translator is IDEADYN written by Aida ${ }^{12}$ which could read the universal files and translate them into LS-DYNA3D input files. The contacts can also be defined inside the preprocessor (I-DEAS Master Series) by giving dummy faces pressures of 
specified values to the faces that could potentially come into contact. Rigid walls can be defined by giving nodal moments, and initial velocities can be given by nodal forces of magnitude equal to the velocity. These initial conditions given in I-DEAS Master Series can be translated by IDEADYN to write a LS-DYNA3D input file. Also, the termination time for the analysis, material properties and rigid wall position vectors can be given at the time of translation from the universal file to the LS-DYNA3D input file. IDEADYN can translate beams, thin shells, tetrahedral elements and brick elements from an I-DEAS universal file to an LS-DYNA3D input file.

LS-TAURUS was the post processor used to analyze the results from LS-DYNA3D output files after the solution. When the solution phase is finished, LS-DYNA3D writes down both ASCII and binary files for viewing the deformation, stress etc., and also plots various graphs for the analysis. LS-TAURUS can read these files and can produce a graphical output for the deformation and stress contours and also plot various graphs for solution. LS-TAURUS has three phases of post processing, the graphics and deformation output phase where the graphic results are post processed, the time history phase where the nodal and elemental component output is obtained as graphs, and the third phase called the ASCII phase where it can read and display the ASCII files and plot graphs for global energy, reaction forces, etc.

Other standard software used for crash analysis of vehicles include LL Dyna3D and PAMCRASH. These codes use the explicit finite element method for solving the system of equations. The standard preprocessors for these packages are Hypermesh, Patran, and ProEngineer, which create the desired geometry, mesh it with the elements and nodes, and write a input file in the standard format. 


\section{CHAPTER 3 - THEORY OF LS-DYNA3D}

\subsection{FEM PRELIMINARIES}

The governing equations of LS-DYNA3D are based on the principle of balance of linear momentum, which expresses the Newtonian concept that the time rate of change of the linear momentum of a particle as measured from a fixed coordinate system must equal the sum of the external forces acting on it. The momentum equation is given by

$$
\sigma_{i j, j}+\rho f_{i}=\rho \ddot{x}_{i} .
$$

Consider a body that moves from a time state $\mathrm{t}=0$ to another position at time $\mathrm{t}=\mathrm{t}$ defined by the coordinates as shown in Figure 3.1 .

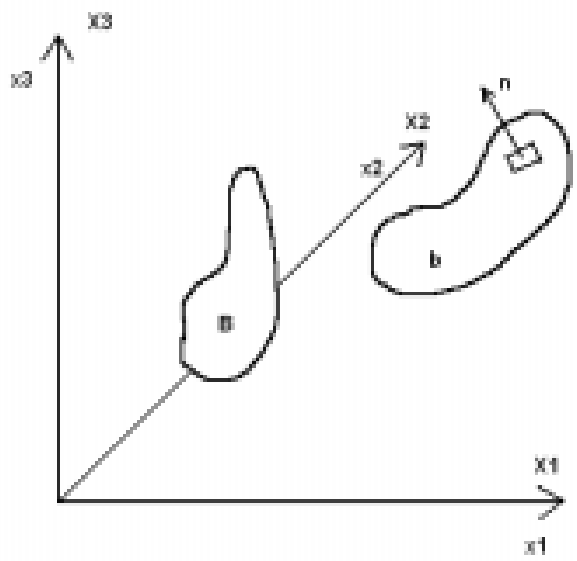

Fig 3.1 Displacement of a body from position $B$ at time $t=0$ to position $b$ at time $t=T$.

The body moves in a fixed reference coordinate system from

$$
\mathrm{X}_{\alpha}(\alpha=1,2,3) \text { to } \mathrm{x}_{\mathrm{i}}(\mathrm{i}=1,2,3) \text {. }
$$


The time dependent deformation can be expressed in terms of $\mathrm{X}_{\alpha}$ and $\mathrm{t}$ as

$$
x_{i}=x_{i}\left(X_{\alpha}, t\right)
$$

based on Lagrangian formulation. The initial conditions describing the body are

$$
\mathrm{x}_{\mathrm{i}}\left(\mathrm{X}_{\alpha}, 0\right)=\mathrm{X}_{\alpha}
$$

and

$$
\dot{\mathrm{x}}_{\mathrm{i}}\left(\mathrm{X}_{\alpha}, 0\right)=\mathrm{V}_{\mathrm{i}}\left(\mathrm{X}_{\alpha}\right)
$$

The equation of linear momentum is given as

$$
\sigma_{\mathrm{ij}, \mathrm{j}}+\rho \mathrm{f}_{\mathrm{i}}=\rho \ddot{x}_{\mathrm{i}} .
$$

The traction boundary conditions are given by

$$
t_{i}(t)=\sigma_{i j} n_{i}
$$

The displacement boundary condition over any boundary $\partial b_{1}$ is

$$
x_{i}\left(X_{\alpha}, t\right)=D_{i}(t)
$$

which denotes any final displacement state.

The contact discontinuity over any boundary $\partial \mathrm{b}_{2}$ is given by

$$
\left(\sigma_{\mathrm{ij}}^{+}-\sigma_{\mathrm{ij}}^{-}\right) \mathrm{n}_{\mathrm{i}}=0
$$


Forces are balanced over and below the boundary.

Using equations 3.1.6, 3.1.7 and 3.1.9 results in

$$
\int_{v}\left(\rho \ddot{x}_{i}-\sigma_{i j, j}-\rho f_{i}\right) \delta x_{i} d v+\int_{\partial b_{1}}\left(\sigma_{i j} n_{j}-t_{i}\right) \delta x_{i} d s+\int_{\partial b_{2}}\left(\sigma_{i j}^{+}-\sigma_{i j}^{-}\right) n_{j} \delta x_{i} d s=0
$$

where the integrations are over the current geometry. Applying the Green's theorem, which relates the surface and volume integrals,

$$
\int_{\partial b 1} \sigma_{i j, j} n_{j} \delta x_{i} d s+\int_{\partial b 2}\left(\sigma_{i j}^{+}-\sigma_{i j}^{-}\right) \delta x_{i} d s=\int_{v} \sigma_{i j} \delta x_{i} d v
$$

where

$$
\left(\sigma_{\mathrm{ij}} \delta \mathrm{x}_{\mathrm{i}}\right)_{\mathrm{j}}-\left(\sigma_{\mathrm{ijj}} \delta \mathrm{x}_{\mathrm{i}}\right)=\sigma_{\mathrm{ij}} \delta \mathrm{x}_{\mathrm{i}, \mathrm{j}}
$$

and a comma denotes a covariant differentiation. Substituting Equation 3.1.11 back into Equation 3.1.10, yields

$$
\delta \pi=\int_{v} \rho \ddot{x}_{i} \delta x_{i} d v+\int_{v}\left(\sigma_{i j} \delta x_{i}\right)_{, j} d v-\int_{v} \rho f_{i} \delta x_{i} d v \int_{\partial b_{1}} t_{i} \delta x_{i} d s=0 .
$$

The above equation gives the statement of principle of virtual work. This is superimposed over a mesh of finite elements to get the strain displacement matrix.

$$
x_{i}=x_{i}\left(X_{\alpha}(\beta, \eta, \Gamma), t\right)=\sum_{j=1}^{k} \phi_{j}(\beta, \eta, \Gamma) x_{i}^{j}(t)
$$

Summing the same over $\mathrm{n}$ elements yields 


$$
\delta \pi=\sum_{\mathrm{m}=1}^{\mathrm{n}} \delta \pi_{\mathrm{m}}=0
$$

From equation 3.1.13, the summation for $\mathrm{n}$ elements in the model as

$$
\sum_{m=1}^{n} \int_{v_{m}} \rho \ddot{x}_{i} \phi_{i}^{m} d v+\int_{v m} \sigma_{i j}^{m} \phi_{i, j}^{m} d v-\int_{v m} \rho f_{i} \phi_{i}^{m} d v-\int_{\partial b 1} t_{i} \phi_{i}^{m} d s=0,
$$

where

$$
\phi_{\mathrm{i}}^{\mathrm{m}}=\left(\phi_{1}, \phi_{2}, \phi_{3}, \ldots \phi_{\mathrm{k}}\right)_{\mathrm{i}}^{\mathrm{m}}
$$

\subsection{Solid Elements}

For a mesh of eight noded solid hexahedron elements, Equation 3.1.14 becomes

$$
x_{i}\left(X_{\alpha}, t\right)=x_{i}\left(X_{\alpha}(\beta, \eta, \Gamma), t\right)=\sum_{j=1}^{8} \phi_{j}(\beta, \eta, \Gamma) x_{i}^{j}(t)
$$

The shape function $\phi_{\mathrm{j}}$ is defined for the 8-node hexahedron as

$$
\varphi_{\mathrm{j}}=\frac{1}{8}\left(1+\xi \xi_{\mathrm{j}}\right)\left(1+\eta \eta_{\mathrm{j}}\right)\left(1+\zeta \zeta_{\mathrm{j}}\right)
$$

where $\xi_{j}, \eta_{j}$, and $\zeta_{j}$ take the nodal values $( \pm 1, \pm 1, \pm 1)$ and $x_{i}^{j}$ is the nodal coordinate of the $\mathrm{j}^{\text {th }}$ node in the $\mathrm{i}^{\text {th }}$ direction as per Figure $3.2^{24}$. 


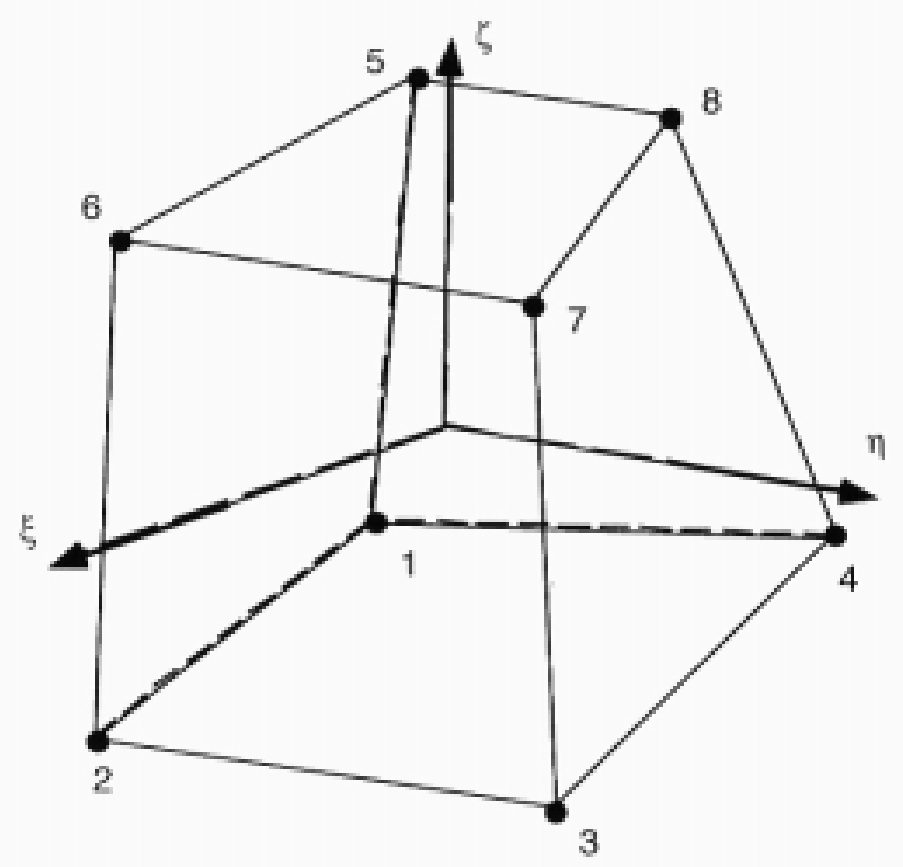

Fig 3.2 Eight noded solid element.

For a solid element, $\mathrm{N}$ is the $3 \times 24$ rectangular interpolation matrix, given by

$$
\mathrm{N}(\xi, \eta, \zeta)=\left[\begin{array}{ccccccccc}
\phi_{1} & 0 & 0 & \phi_{2} & 0 & 0 & \ldots . . & 0 & 0 \\
0 & \phi_{1} & 0 & 0 & \phi_{2} & 0 & \ldots . & \phi_{8} & 0 \\
0 & 0 & \phi_{1} & 0 & 0 & \phi_{2} & \ldots . & 0 & \phi_{8}
\end{array}\right]
$$

The greatest advantage of single point integration is the substantial savings in CPU time. The time spent on evaluating the constitutive relations is reduced by a factor of eight whereas the eight point integration takes eight times the time as single point integration. A disadvantage with eight point integration is that when it is used in the solution of plasticity problems and other problems where the Poisson's ratio approaches 0.5 , the elements tend to lock up in the constant volume bending modes. To avoid such a locking, an average pressure must be used over the elements, and hence the zero energy modes are resisted by deviatoric stresses. 


\subsection{Hour GLASS CONTROL}

A disadvantage of single point integration is the need to control the zero energy modes otherwise called the hourglassing modes. These hour glass modes arise when they have periods that are much shorter than the periods of structural response. The easiest way of stopping this mode is by giving a small viscous damping or a small resistive elastic stiffness capable of stopping the modes. The hourglass modes are made orthogonal to the real deformation and hence the work done by the hourglass modes is neglected. LS-DYNA3D has three kinds of hourglassing defined in it. Standard LSDYNA3D hourglassing takes the least amount of time followed by the FlanaganBelytschko hourglassing and Wilkins FDM hourglassing. Table $3.1^{31}$ shows the comparison among the methods for the operation count for constant the stress hexahedron. This includes the additions, subtractions, multiplications, and divisions in the major subroutine and it is independent of vectorization.

Table 3.1 Operation count for hourglassing types.

\begin{tabular}{|c|c|c|c|}
\hline Component & Standard & Flanagan-Belytschko & Wilkins FDM \\
\hline Strain displacement matrix & 94 & 357 & 843 \\
\hline Strain rates & 87 & 156 & - \\
\hline Force & 117 & 195 & 270 \\
\hline Subtotal & 298 & 708 & 1,113 \\
\hline Hourglass control & 130 & 620 & 680 \\
\hline Total & 428 & 1,328 & 1,793 \\
\hline
\end{tabular}

\subsection{TIME INTEGRATION}

Consider the single DOF damped system shown in the Figure $3.3^{15}$. The free body diagram for the system is shown in Figure $3.4^{15}$. Applying the D'Alembert's principle, the governing equations of the system are written as 


$$
\mathrm{F}_{\mathrm{s}}+\mathrm{F}_{\mathrm{D}}+\mathrm{F}_{\mathrm{I}}=\mathrm{p}(\mathrm{t}) .
$$

The inertia force can be written as

$$
\mathrm{F}_{\mathrm{I}}=\mathrm{M} \ddot{\mathrm{u}},
$$

where the acceleration is given as

$$
\ddot{u}=\frac{d^{2} u}{{d t^{2}}^{2}} .
$$

The damping force is given as

$$
\mathrm{F}_{\mathrm{D}}=\mathrm{c} \dot{\mathrm{u}}
$$

The velocity is

$$
\dot{\mathrm{u}}=\frac{\mathrm{du}}{\mathrm{dt}} .
$$

The force due to the elastic spring is

$$
\mathrm{F}_{\text {int }}=\mathrm{ku} \text {. }
$$

The equations of motion for linear behavior gives the linear O.D.E:

$$
M \ddot{u}+c \dot{u}+k u=p(t) .
$$

The equations for the non-linear behavior give the internal force as a nonlinear function of the displacement leading to non-linear O.D.E 


$$
M \ddot{u}+c \dot{u}+F_{I}(u)=p(t) .
$$

For linear O.D.E closed form solutions are possible but for non-linear O.D.E only numerical solutions are possible. LS-DYNA3D uses the explicit central time difference scheme to integrate the equations of motion.

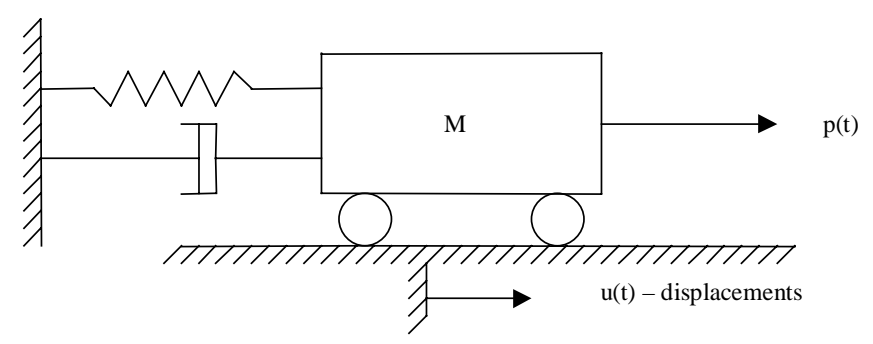

Fig 3.3 Simple spring mass system with a damper.

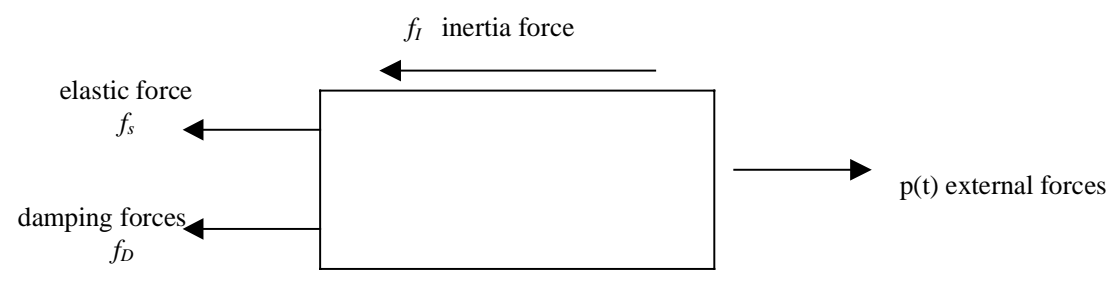

Fig 3.4 Free body diagram of the spring mass system.

\subsection{Central Difference Method}

The equations of the motion at any time $\mathrm{n}$ are given as

$$
M a^{n}=P^{n}-F^{n}+H^{n} .
$$

To advance to time $\mathrm{t}^{\mathrm{n}+1}$, LS-DYNA3D uses the central time integration:

$$
\mathrm{a}^{\mathrm{n}}=\frac{\left(\mathrm{P}^{\mathrm{n}}-\mathrm{F}^{\mathrm{n}}+\mathrm{H}^{\mathrm{n}}\right)}{\mathrm{M}}
$$


The velocity is calculated by

$$
v^{n+1 / 2}=v^{n-1 / 2}+a^{n} \Delta t^{n}
$$

and the displacement by

$$
u^{n+1}=u n+v^{n+1 / 2} \Delta t^{n+1 / 2}
$$

where

$$
\Delta \mathrm{t}^{\mathrm{n}+1 / 2}=\frac{\left(\Delta \mathrm{t}^{\mathrm{n}}+\Delta \mathrm{t}^{\mathrm{n}+1}\right)}{2}
$$

The variables $\mathrm{v}$ and $\mathrm{u}$ are the global nodal and velocity and displacement vectors, respectively. The displacement increments are added to the initial geometry giving

$$
x^{n+1}=x^{0}+u^{n+1}
$$

where $\mathrm{x}^{0}$ is the initial position and $\mathrm{x}^{\mathrm{n}+1}$ is the current position.

\subsection{NUMERICAL ANALYSIS}

Numerical analysis in general attempts to solve the mathematical problems like differential equations by numerical procedures, which could be programmed using a standard programming language. The differential equation can be split into numerical components in the time axis using the forward, central or the backward differentiation methods and could be programmed to determine the higher time step values from the initial and boundary values. The numerical methods can be broadly classified as the explicit and the implicit methods. The explicit method calculates the next time step value using the previous time step values, whereas the implicit method calculates the next time 
step values by solving a matrix of the present and the previous time step values. The explicit method requires shorter time step for an accurate solution, whereas the implicit methods can give reliable results with larger time steps. Also, most of the implicit methods are unconditionally stable, whereas the explicit methods are mostly conditionally stable.

\subsubsection{LINEAR AND NON-LINEAR ANALYSIS}

A linear analysis assumes that the stiffness matrix in the finite element formulation is linearly elastic and always obeys Hook's law. The material properties that are assumed in this kind of analysis would be infinitely elastic.

A non-linear analysis basically can be classified into two types called the geometric non-linearity and the material non-linearity. Geometric non-linearity is assumed in the places where there is a large displacement in the finite element model such as the deflection in a string or the deflection in a long circular beam where the $1 / \mathrm{d}$ ratio is very high. Some finite element packages also take care of the change in the geometry of the cross section and the shear deflections in this case. Material non-linearity occurs in places where the material has a finite stress-strain proportionality limit, after which Hook's law is no longer valid. In this kind of finite element analysis, the elastic plastic stress-strain curve is given as an input to the material property of the elements. Once the stress-strain curve is given as a input to the material property the stiffness matrix is updated accordingly. 


\subsection{CONTACT IMPACT ALGORITHM IN LS-DYNA3D}

LS-DYNA3D has three algorithms for contact defined as the kinematic constraint method, the penalty method, and the distributed parameter method. The kinematic constraint method is used only for tying interfaces. Interfaces are defined in three dimensions; one side of the interface is called the master surface and the other is called the slave surface. By the kinematic constraint method, constraints are imposed on global equations by a transformation of the nodal displacement components of the slave nodes along the contact interface. This transformation has the effect of eliminating the normal degrees of freedom of the nodes. Impact and release conditions are imposed to insure momentum conservation. This method has some problems when the master surface is finer than the slave surface. In this case, the master nodes can penetrate into the slave surface without resistance. One way to avoid this problem is to have a similar mesh for the slave surface and the master surface.

The penalty method is used in explicit programs like LS-DYNA2D and LSDYNA3D as well as implicit programs like NIKE2D and NIKE3D ${ }^{17}$. This method consists of placing normal interface springs between the master and the slave surface. This method is found to excite if hourglassing occurs and the interface stiffness is chosen to be approximately the same order of magnitude as the stiffness of the interface element normal to the interface. If the interface stiffness is too large or too small, penetration may occur and scaling up and down the penalty stiffness value can solve this problem. This method increases the number of time steps and the CPU time compared to the previous method. 
The distributed parameter formulation involves sharing one half of the slave element mass of each element in contact to the master surface area. After completing the distribution of the mass, constraints are imposed on the slave node accelerations and velocities to ensure their movement along the master surface.

Slave search is common to all the contact algorithms discussed above in LSDYNA3D. This search finds the nearest point on the master surface for each slave node. Lines are drawn from a slave node to the nearest point that will be perpendicular to the master surface. In contact type 3 in LS-DYNA3D, which is called the sliding with closure and separation, each slave node is checked for penetration through master surface. If the slave nodes do not penetrate, nothing is done. If the slave nodes penetrate, an interface force is applied proportional to the amount of penetration in addition to the interface stiffness.

Shell thickness can also be taken into account when the contact is defined between shell elements. This is particularly useful in the case of sheet metal forming operations. Unless thickness is considered, in the contact, the effect of thinning on fractional interface stresses due to the membrane stretching will be difficult to treat. For considering the thickness, the slave and the master surface projections are based from the mid surface of the shell element. The surfaces, therefore, should be offset by a distance equal to half the total thickness.

Tied interfaces are useful for joining two meshes of incompatible mesh sizes. This feature can decrease the effort required to reduce the mesh sizes to match two different surfaces of different element lengths. In this method the constraints are imposed only on 
the slave nodes, and it is therefore essential to define the more coarsely meshed side of the interface as the master surface. 


\section{CHAPTER 4 - FINITE ELEMENT MODEL OF THE BUS}

\subsection{InTRODUCTION TO FEM Procedure}

The development of a finite element model for crashworthiness can be divided into three stages. Each stage has to be followed one after the other in any standard finite element software package or in a user written code for finite element method. They are shown in the Figure 4.1.

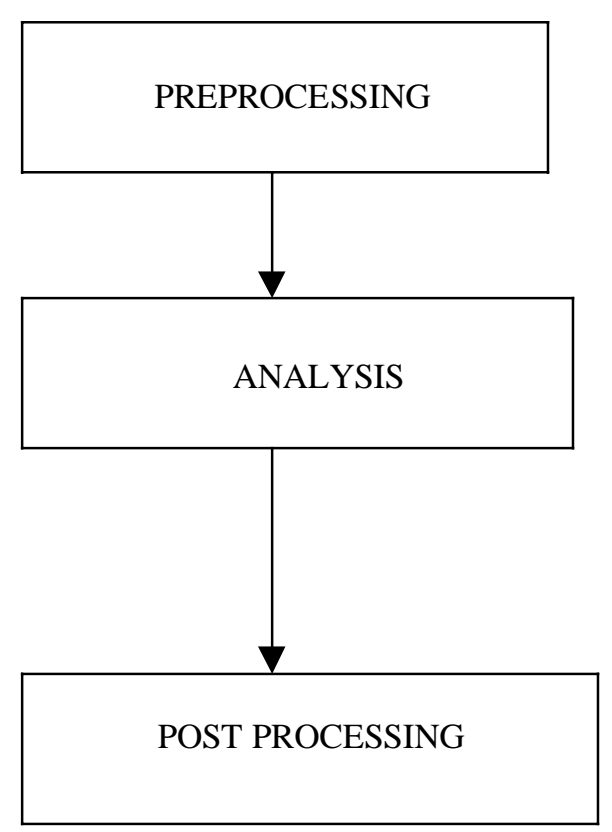

Fig 4.1 Flow chart of the finite element method.

The first stage in the development of the model is the pre-processing. In this stage, the model is built using desired elements to the exact or the approximate geometry of the actual component. All the properties of the elements such as physical and material properties are given in this stage. Various pre-processors like I-DEAS, LS-INGRID, PATRAN, are available for LS-DYNA3D; I-DEAS was chosen to build the model in this 
case. I-DEAS has excellent capabilities for pre-processing and the model can be transferred to the LS-DYNA3D input format using standard translators. These translators read the universal file, translate the data, and write an input file for LS-DYNA3D, which can be directly submitted for analysis. The physical properties and the material properties can also be translated from the universal file to the LS-DYNA3D input file.

Once the pre-processing is done, the model is submitted to LS-DYNA3D for analyzing the model to create the output. The analysis results will be stored as a series of files and can be given a user-specified name. Most of the files will be in binary format, and these files contain the results of the analysis and cannot be viewed or understood without having the software that translates them and gives a graphical output. This stage is called the post-processing where the standard results like stress, displacement, velocity, and acceleration are obtained. As in any design or analysis problem, the modeling strategy may change. From the results in the post processor, the model may have to be adjusted to meet the experimental or the numerical requirements.

\subsection{Pre-Processing}

The model of the school bus was built in I-DEAS Master Series 4.0 using various modules of the software such as Master Modeler, Master Surfacing, Meshing and Boundary Conditions. The advantage with this software is that the model can be transferred from one module to the other easily and there are many tools available for free and mapped meshing. 


\subsubsection{Data Collection}

To start a model for pre-processing, the first step is to measure the geometry of each component whose finite element model has to be built. The simplest way to get the dimensions of the component is to get all the component drawings from the company that manufactures the bus or to get a service manual gives the assembly of the components of the bus. Drawings were obtained from a manufacturer of a school bus that is used for student transportation at West Virginia University and thousands of school districts throughout the United States. Along with the drawings, the measurements of the school bus were taken by manually measuring each component of the bus using a measuring tape, Vernier caliper, and a ruler. Also, pictures of various components of the bus and the assembly of the components were taken with a digital camera and used for assembling the model.

\subsubsection{PRE-PRoceSSING IN I-DEAS}

Obviously the finite element model of all the components of the bus cannot be developed due to time constraints and unnecessary usage of resources like memory and computing time for a non-significant increase in the solution accuracy. The components that are to be modeled by a finite element mesh must be prioritized. Prioritization was based on aspects like energy absorbing capability, involvement in the collision event, and material type.

Table 4.1 shows the prioritization of the components for frontal impact. All the components listed in Table 4.1 were modeled using I-DEAS. Priority 1 has the highest priority and Priority 3 the lowest. The rear axle and the body sheet do not play an important role in the frontal impact and hence they were not considered for prioritization. 
During meshing, the distortion and stretch of the elements were controlled and brought down below 0.7. The warp angle of all the shell elements was reduced below the allowable limit of 20 degrees in LS-DYNA3D.

Table 4.1 Prioritization of the components for modeling.

\begin{tabular}{|c|c|c|c|c|}
\hline COMPONENET & PRIORITY 1 & PRIORITY 2 & PRIORITY 3 & MATERIAL \\
\hline Engine & $\mathrm{X}$ & & & Cast Iron \\
\hline Radiator & $\mathrm{X}$ & & & Steel \\
\hline Bumper & $\mathrm{X}$ & & & Steel \\
\hline Hood & $\mathrm{X}$ & & & Steel \\
\hline Fender & & $\mathrm{X}$ & & Steel \\
\hline Fire Wall & & $\mathrm{X}$ & & Steel \\
\hline Front Axle & & $\mathrm{X}$ & & Cast Iron \\
\hline Gear Box & & & $\mathrm{X}$ & Cast Iron \\
\hline Radiator Mount & & & $\mathrm{X}$ & Cast Iron \\
\hline Frame & & & $\mathrm{X}$ & Steel \\
\hline
\end{tabular}

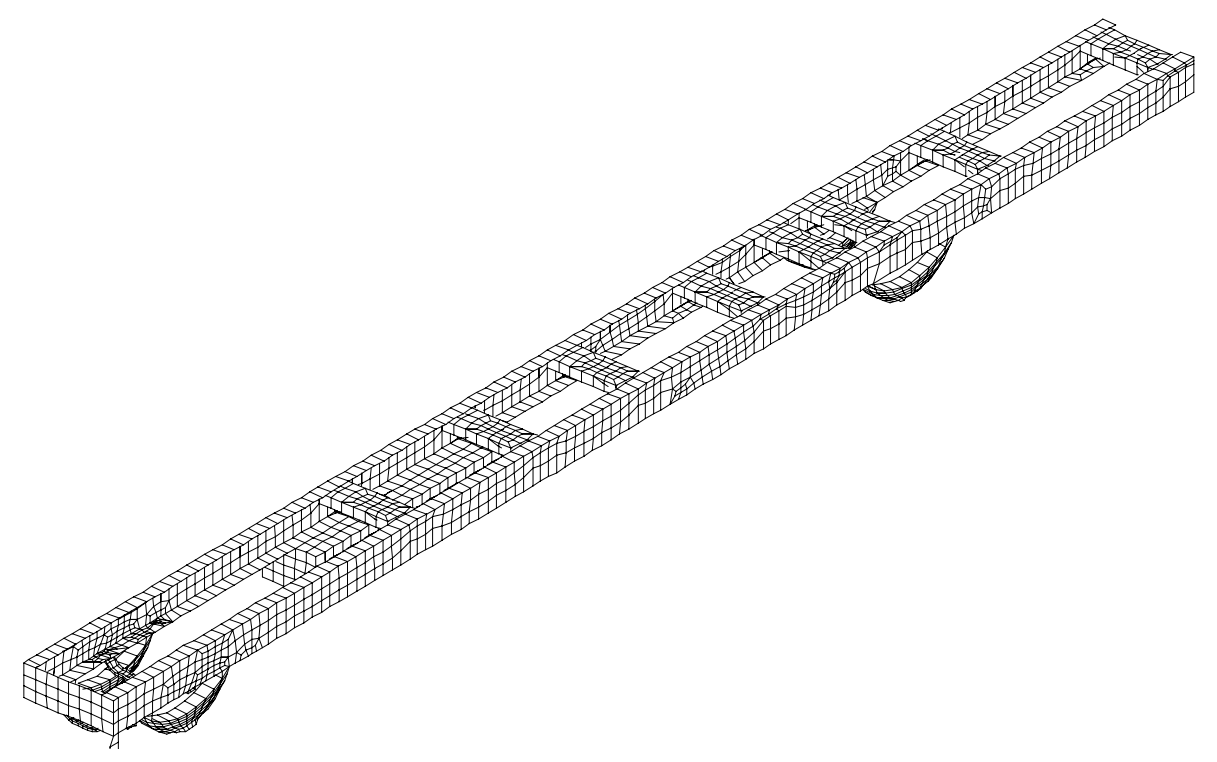

Fig 4.2 Frame structure of the bus with springs and fuel tank. 
The frame is the main structural member in the bus chassis on which the entire bus load is transferred from the body of the bus. The picture of the frame along with the leaf springs and the fuel tank is shown in Figure 4.2. The frame consists of two main members that have a standard channel up to six mm thick made of mild steel. The main members are connected by a series of cross members from the front end of the frame to the rear end. The cross members are fixed to the frame by rivets, which were modeled as rigid connections in the finite element model. The cross members provide a torsion resistance to the frame along with bending strength. The frame was modeled using thin shell elements and the thickness of the elements were specified in the physical property of the elements in the LS-DYNA3D input file. The leaf springs were modeled using brick elements, and they were connected to the frame by merging the nodes of the spring and the frame. The sliding effect of the shackle was not modeled since it is assumed that it does not play a significant role in the crash scenario. The fuel tank was also modeled using thin shell elements that are attached to the frame. The weight of the fuel was given as point loads on the tank.

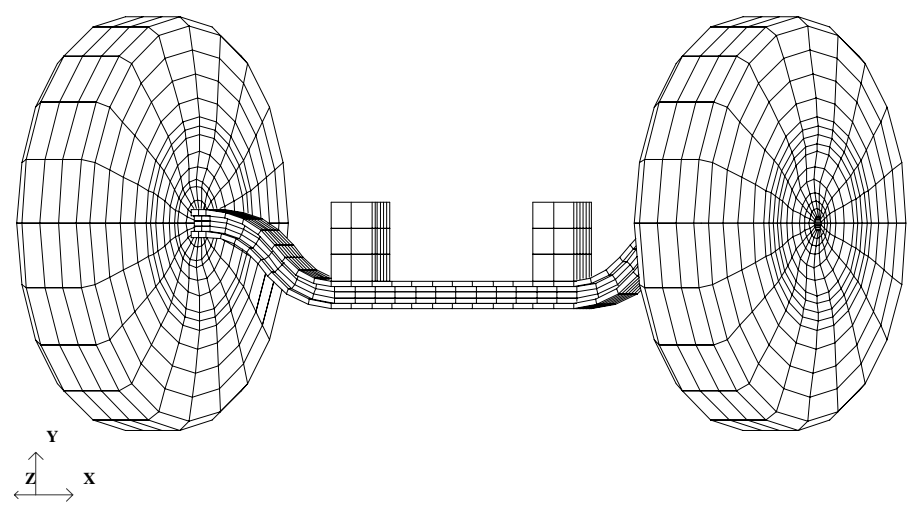

Fig 4.3 Front axle with tires. 
The front axle was modeled using brick elements with the cross section measured. The front axle was rigidly connected to the tires. The front axle beam is made of cast iron and the properties of the cast iron were specified in the LS-DYNA3D input file. The element length for the front axle was fixed as $20 \mathrm{~mm}$. The picture of the front axle with the tires is shown in Figure 4.3. The tires were modeled using brick elements. The elastic properties of the tire were assumed since it is assumed that it does not play an important role in the crash event or the energy absorption except that it provides stability for the roll behavior of the complete bus in a side impact; side impact was not modeled for this work.

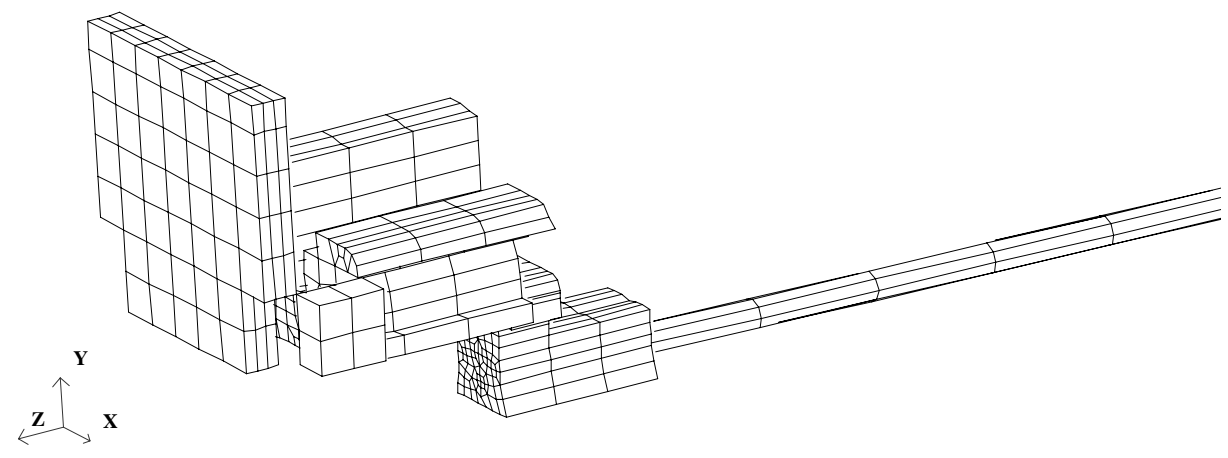

Fig 4.4 Radiator, engine and gearbox with propeller shaft.

The engine and the gearbox are seated in the front end of the frame and they play an important role in the frontal crash event. The finite element model of the engine along with the gearbox and radiator is shown in Figure 4.4. The radiator is fixed in front of the engine and the gearbox is fixed to the frame. The radiator was modeled using thin shell elements to approximate the honey comb structure of the radiator. The engine and the gearbox were modeled as a solid block of mass with the outer dimensions measured from 
the vehicle. The engine and the gearbox were modeled using brick elements and the gearbox was connected to the engine directly at the place where the clutch housing connects the gearbox and the engine. The propeller shaft was also modeled using thin shell elements and connected to the gearbox at one end and to the differential casing of the rear axle at the other end. In the actual vehicle, the propeller shaft is connected to the frame by bearings to prevent the bending of the shaft by its own weight. But the bearings were not taken into account while modeling the propeller shaft since its mass is negligible. The finite element mass of the engine and the gearbox matched well with the actual mass. The center of gravity of the engine and the gearbox was assumed. The actual mass of the engine is $370 \mathrm{~kg}$ and the mass of the finite element model of the engine is $430 \mathrm{~kg}$. The excess mass of $60 \mathrm{~kg}$ was given for the weights of other engine components mounted on the engine like the oil and air filter, and brake oil containers etc. The mass of the actual gearbox is $94 \mathrm{~kg}$ and the mass of the finite element model of the gearbox is 100 $\mathrm{kg}$.

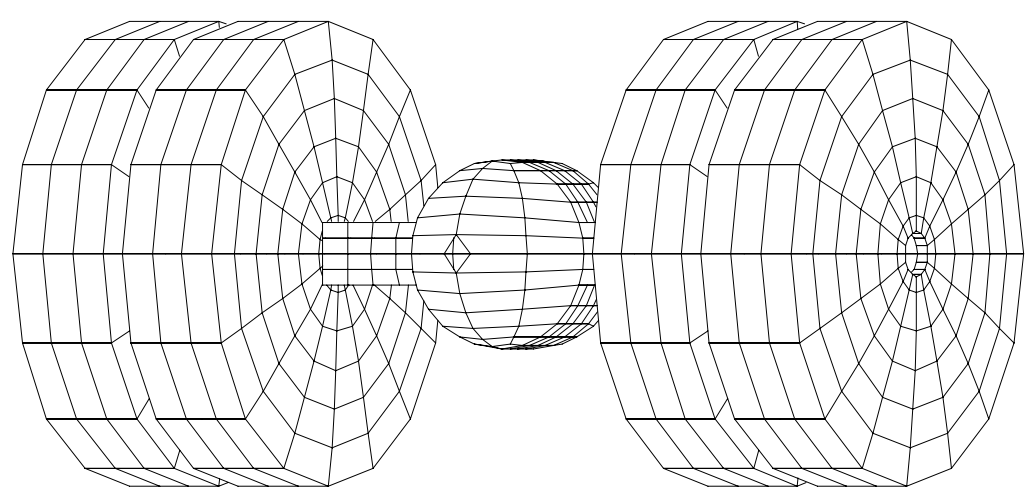

Fig 4.5 Rear axle with tires. 
The rear axle assembly consists of the axle casing, differential casing with the differential gears, axle shafts, and the wheel and tires. The picture of the finite element model of the rear axle is shown in Figure 4.5. The differential casing and the axle casing were modeled as thin shell elements and the differential casing is connected to the propeller shaft at the front end. The differential gears and the axle shafts were modeled as blocks of small masses to account for the weight, inertia, and the center of gravity during the collision event. The tires were modeled as brick elements and were rigidly connected to the axle shafts. The spring seats in the bottom leaf of the rear leaf springs sit on the space between the tires, and the differential casing and the upper ends of the leaf spring are connected to the frame. The sliding between the leaves in the leaf spring and the shackle in the rear end of the leaf springs was not modeled.

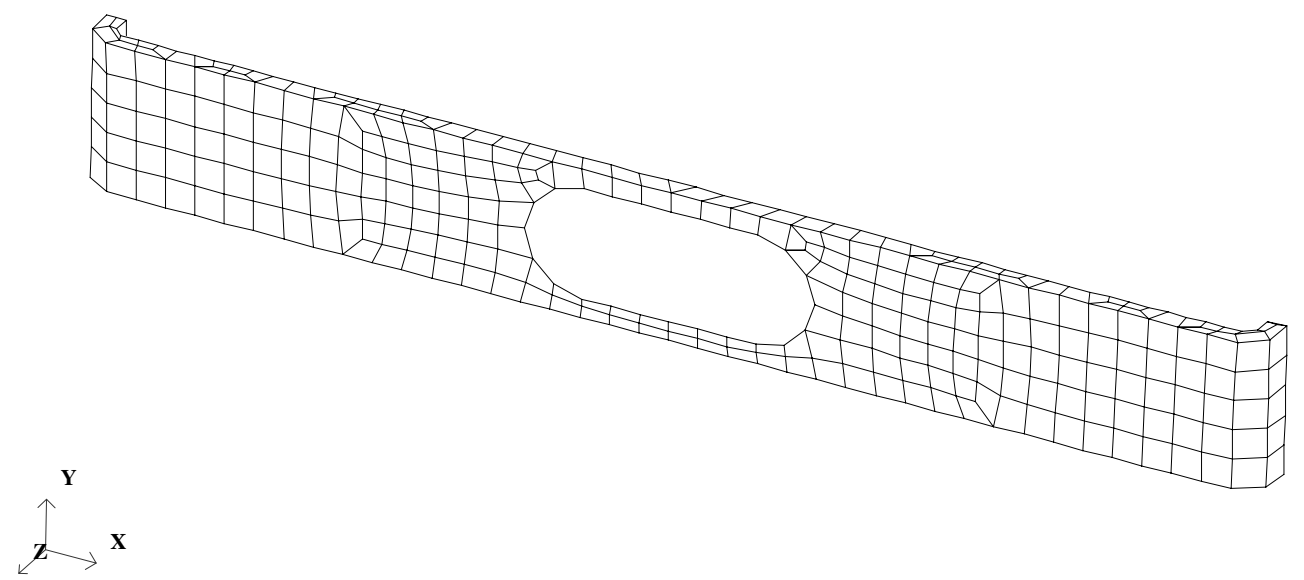

Fig 4.6 Bumper finite element model.

The bumper is a steel structure that is fixed in the front of the frame, and it is an important energy-absorbing component in frontal impact. The picture of the bumper is shown in Figure 4.6. The bumper structure was meshed using thin shell elements, and the 
thickness of the structure was specified in the physical property of the elements in LSDYNA3D input file.

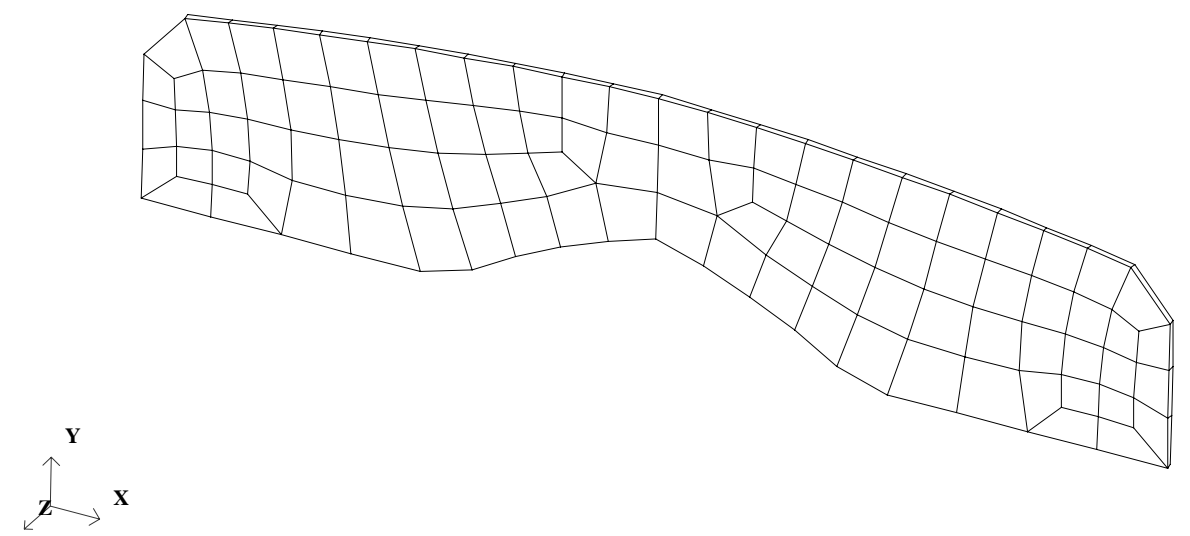

Fig 4.7 Fire wall finite element model.

The firewall helps to prevent the engine and other front end components from entering the driver occupant area in the event of a frontal crash. The picture of the firewall is shown in Figure 4.7. The firewall was meshed using solid brick elements with the thickness of the sheet metal. Solid elements were chosen to mesh the firewall because the potential contacting component to this firewall in the event of a frontal crash is the engine and the engine was made of brick elements. Solid elements were chosen to model the firewall instead of shell elements since LS-DYNA3D can establish very good contact between solid faces than the contact between a solid and a shell element face. 


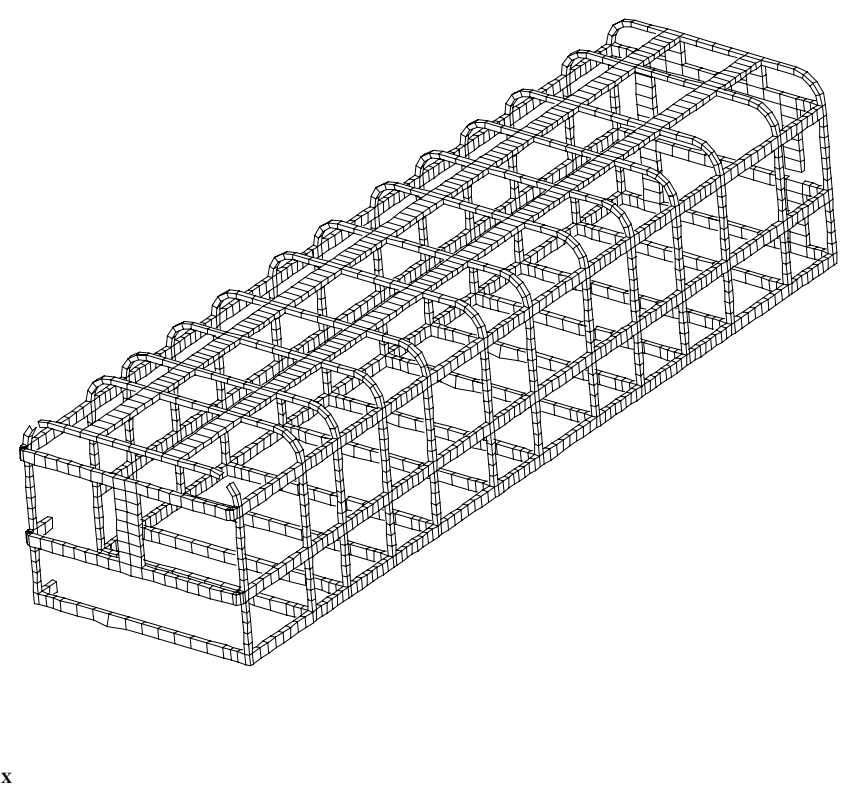

Fig 4.8 Bus body structure.

The bus body structure is built over a skeleton of ribs that gives the structural integrity to the bus body. The sheet metal is then welded or riveted to the ribs to give the entire shape to the body. The picture of the finite element model of the bus skeletal structure is shown in Figure 4.8. The rib structure was meshed using solid elements, and the thin shell sheets were then attached to the solid elements. The floor structure is also attached to the ribs that run over the frame. The cross section of the rib structure is assumed to be square and the dimensions were measured from the field. These rib structures play an important role in giving structural integrity to the bus body during impact. 


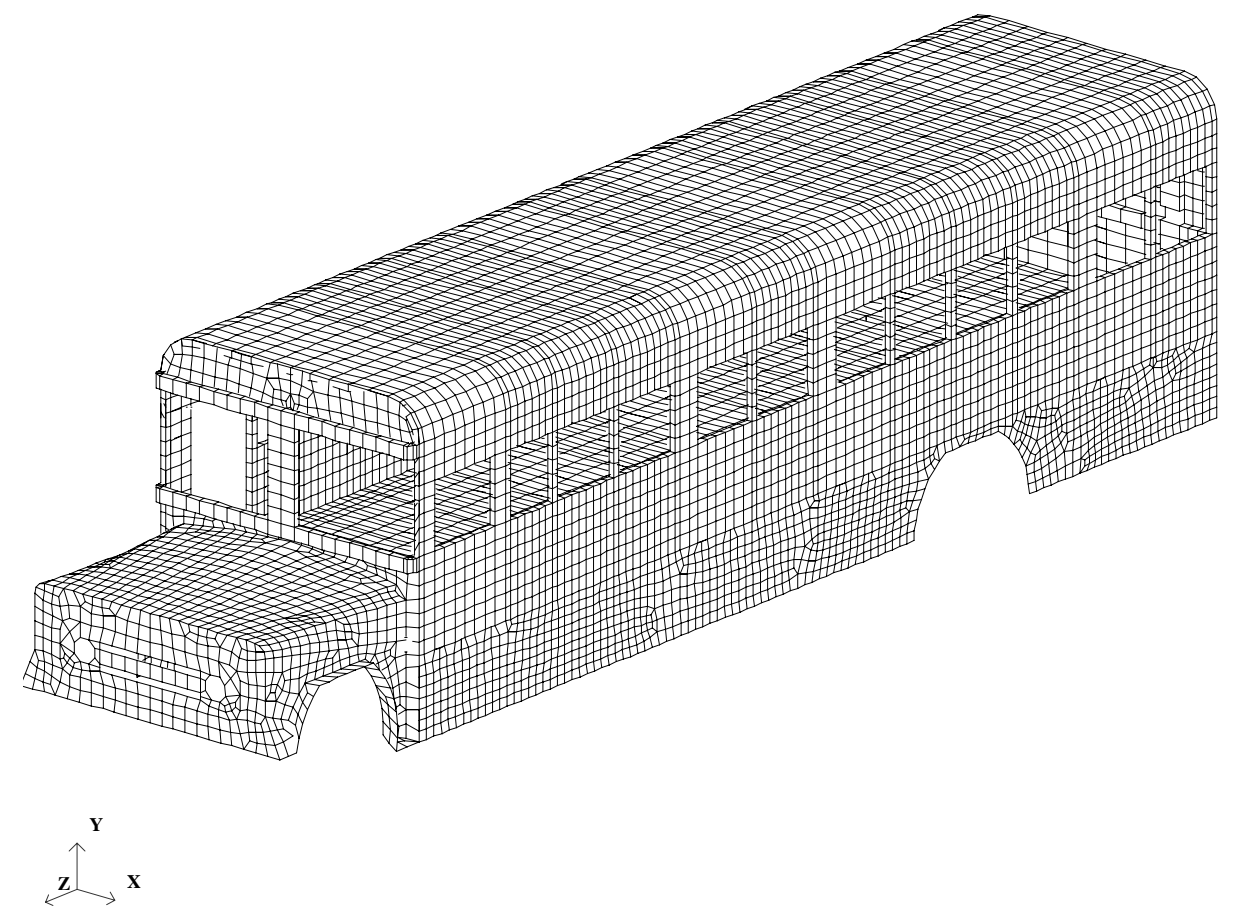

Fig 4.9 Bus body with sheet metal over the ribs.

The body of the bus is made up of steel sheets that form two layers inside and outside and are fixed to the ribbed structure. The finite element model of the bus body with the hood structure is shown in Figure 4.9. The hood and the fender were modeled using thin shell elements. The floor structure is also modeled using thin shell elements and is fixed to the floor ribs, which is again connected to the frame using connecting struts. The connecting struts were modeled as thin shell elements and assumed to be mild steel. The thickness of the sheet structure is specified in the physical property of the elements in the LS-DYNA3D input file. The windshield glasses and the side window glasses were not modeled.

The independent finite element models discussed above were then assembled into a complete finite element model of the bus. Nodes were merged where rigid connections 
are to be made and connecting struts were used to connect the bus body and the chassis. The picture of the complete finite element model of the bus is shown in Figure 4.10.

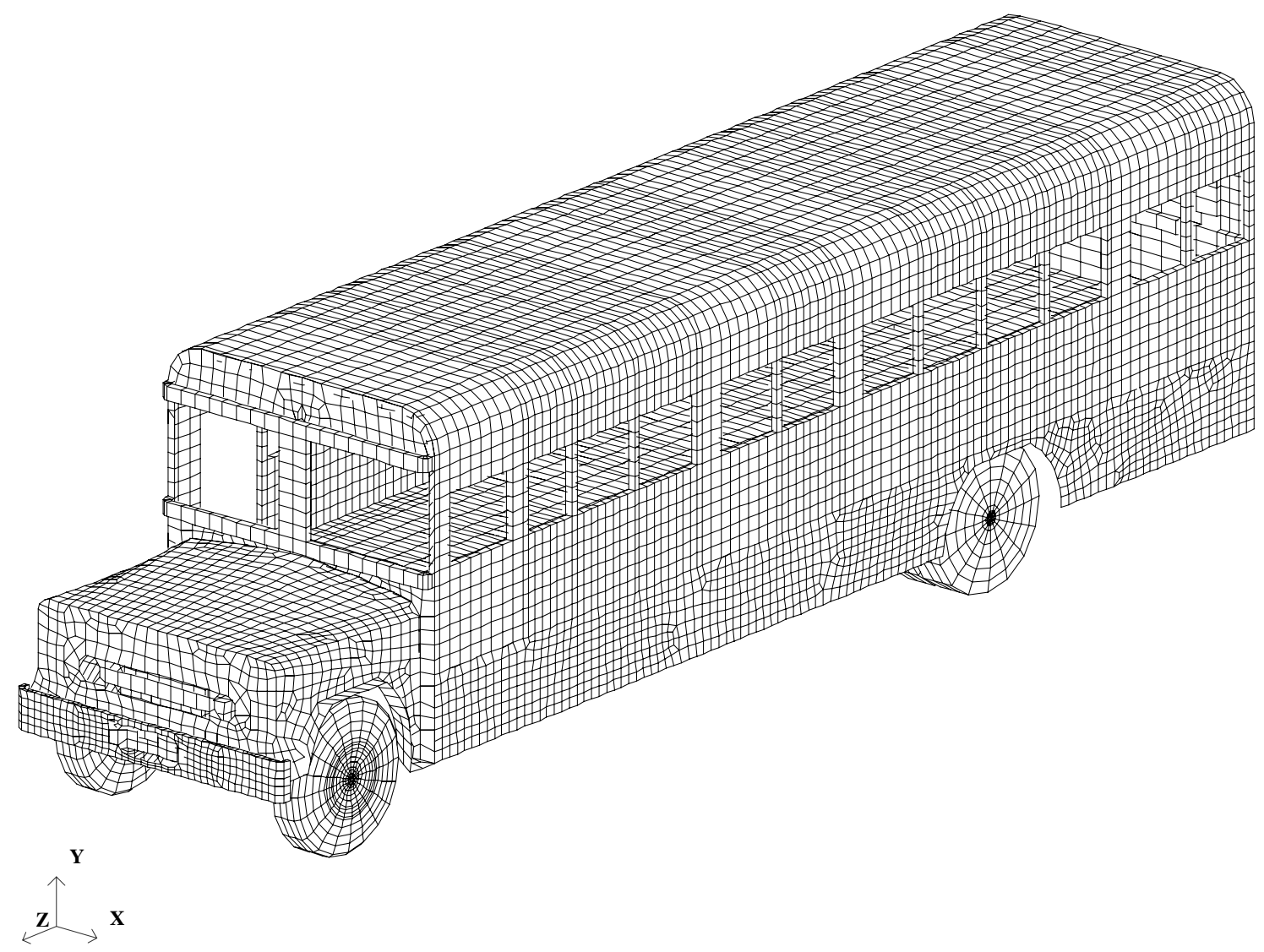

Fig 4.10 Complete finite element model of the bus with different parts assembled.

Component wise mesh density for the complete finite element model is discussed in Table 4.2. This table shows that the a major percentage of the elements lie in the body of the bus, since it has two layers of thin sheets of thickness 22 GA. The tire model has next highest number of elements in the entire model. The engine and gearbox have less number of elements since the element length in that geometry is higher. The hood has a closer mesh, to examine the deformation shape during frontal impact. 
Table 4.2 Component wise mesh density.

\begin{tabular}{|c|c|}
\hline COMPONENET & NUMBER OF ELEMENTS \\
\hline Frame & 2450 \\
\hline Front Axle & 840 \\
\hline Tires & 2200 \\
\hline Rear Axle & 504 \\
\hline Gear Box & 231 \\
\hline Springs & 224 \\
\hline Radiator \& Propeller Shaft & 415 \\
\hline Fuel Tank & 436 \\
\hline Engine & 304 \\
\hline Body Sheet Metal & 10014 \\
\hline Ribs & 1704 \\
\hline Radiator Mount & 30 \\
\hline Bumper & 386 \\
\hline Hood & 888 \\
\hline Fire Wall & 92 \\
\hline
\end{tabular}

The elements of different parts were assigned different material identities even though they had the same material properties. This was done because LS-TAURUS, the post processor, can differentiate between element groups based only on the material identity. This is particularly useful to plot the energies for different parts of the finite element model.

\subsubsection{BOUNDARY CONDITIONS}

Once the finite element model is built and the different parts are assembled, the boundary conditions can be applied to the model in I-DEAS. The following boundary conditions were applied to the bus finite element model.

1. Initial nodal velocities.

2. Sliding interface contact definition.

3. Rigid wall definition. 
I-DEAS does not have a provision for writing the boundary conditions for LSDYNA3D. The translator IDEADYN was used to translate the universal file written from I-DEAS to LS-DYNA3D input deck. IDEADYN is a FORTRAN program that reads the universal file and writes the nodal data, elemental data, boundary conditions, and the initial conditions in the formatted input type of LS-DYNA3D. The nodal velocity, which was the initial condition, was given as nodal forces of magnitude equal to the initial velocity to all the nodes in I-DEAS. When the universal file is written, IDEADYN reads the nodal forces and converts them as initial conditions for the nodes in the LS-DYNA3D input program. The rigid walls are defined for nodes and are given as nodal moments in I-DEAS. IDEADYN reads the nodal moments from the universal file and writes them as the nodes that come into contact with a rigid wall in the LS-DYNA3D input file. A rigid wall definition means the nodes that are defined for the rigid wall are the slave nodes and the rigid wall is the master surface. That means that the nodes that are defined for the rigid wall contact cannot penetrate into the rigid wall. By varying the magnitude of the dummy nodal moments, up to 20 rigid walls can be defined using IDEADYN. The head and the tail of the normal vector for the rigid wall can be defined during the translation process.

Contact type 3 in LS-DYNA3D, called the sliding and general interface contact was defined in the front end of the vehicle. It is defined as face pressures in I-DEAS and the translator writes them into the LS-DYNA3D input deck. The master surfaces are defined with a positive force and the slave surfaces are defined with negative forces. Care should be taken that the shell element normals are oriented in the correct direction for all the elements. By varying the magnitude of the dummy face pressures, up to 11 contact types 
can be defined in I-DEAS and translated into the LS-DYNA3D input file. Another contact type defined in this finite element model is the self-contact between the same material. This happens when the material folds and comes into contact with some other section of the same material. This type of contact was defined for the elements of hood in the frontal impact and the elements on the bus body sheet metal at the rear portion for the rear impact. This type of contact requires only the slave surface and requires more CPU time since all nodes are checked to find the nearest node for contact. The translation process is shown as a flow chart in Figure $4.11^{13}$.

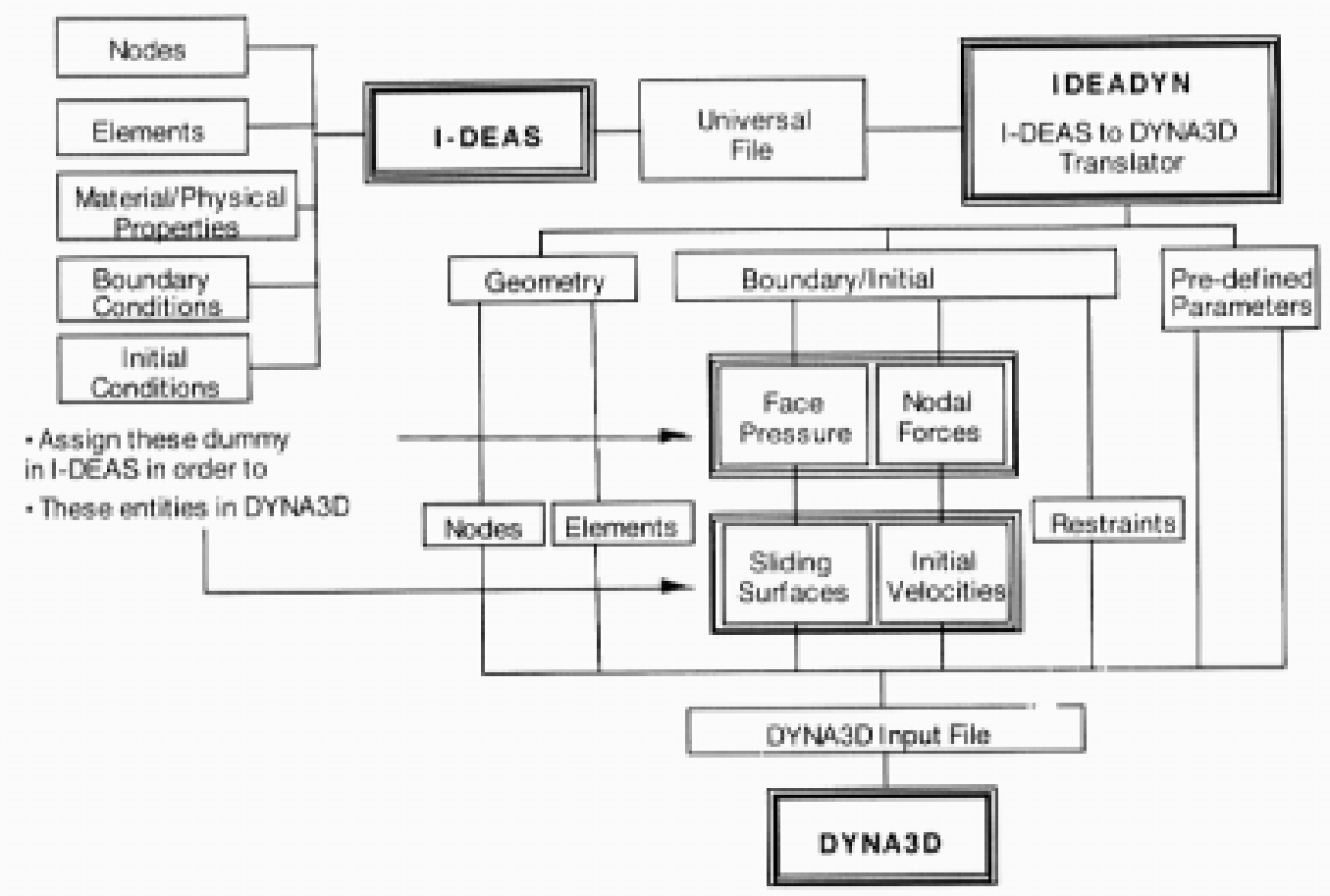

Fig 4.11 Flow chart for file translation from I-DEAS to LS-DYNA3D.

Before writing the universal file from I-DEAS, the nodes and elements have to be renumbered to start from one and end at the number of nodes because the nodes and the elements are written down in the same order as the universal file. LS-DYNA3D cannot 
understand if the nodes and the elements are written down in an arbitrary order. This process is done in the meshing module of I-DEAS, where the elements and the nodes are renumbered starting from one to the maximum number of nodes or elements.

\subsection{ANALYSIS}

The analysis of the bus model was performed in LS-DYNA3D. LS-DYNA3D is a completely vectorized code, which could perform transient nonlinear dynamic analysis of

finite element models. The memory allocation is dynamic and it has several material types that can be defined for modeling. The limiting factor for the allocation of memory for the number elements, nodes, boundary conditions, and initial conditions is the memory of the computer. The interactive execution for this program in the command prompt is as follows:

\section{LS-DYNA3D I=input file $\mathrm{O}=$ output file $\mathrm{F}=$ thf.}

where $\mathrm{I}$ is the user specified input file, $\mathrm{O}$ is the output file and $\mathrm{F}$ is the time history file.

Similarly, a number of output files can be specified for output. Unless specified, the default file for the error messages is taken as "messag". Any kind of errors encountered during the solution run is written down in this ASCII file and can be viewed. The status of the solution can be known by using the control switches in the software. Table 4.3 lists the control switches available and their functions. Two UNIX DEC Alphas, one with 064 chip, $289 \mathrm{MHz}, 256 \mathrm{MB}$ RAM and the other with 164 chip, $500 \mathrm{MHz}, 1000 \mathrm{MB}$ Ram were used to solve the finite element models in LS-DYNA3D. LS-DYNA3D version 940.1 was used on both the computers. 
Table 4.3 Control switches for LS-DYNA3D.

\begin{tabular}{|c|c|}
\hline Type & Response \\
\hline sw1 & A restart file is written and LS-DYNA3D terminates. \\
\hline sw2 & LS-DYNA3D responds with time and cycle numbers. \\
\hline sw3 & A restart file is written and LS-DYNA3D continues. \\
\hline sw4 & A plot state is written and LS-DYNA3D continues. \\
\hline sw5 & Interactive graphic phase. \\
\hline stop & Terminate execution immediately. \\
\hline
\end{tabular}

\subsubsection{MATERIAL MODELS IN LS-DYNA3D}

There are currently 126 material models and 11 equation of state forms in the 940.1 version of LS-DYNA3D. The discussion below is curtailed to material model that has been used in this finite element model.

\subsubsection{MATERIAL 1}

This material model is for elastic materials. This material model was used for tires since the deformation of the tires is assumed to have a minimum effect on impact. The elastic material assumes that the material is infinitely elastic and stays in the proportional limit and obeys Hooks law always. The variables in this material model are Young's modulus, Poisson's ratio, the axial damping factor and the bending damping factor for beams. By setting the appropriate flag, this model can behave like a fluid where the bulk modulus, $\mathrm{K}$, and the pressure rate, $\dot{\mathrm{p}}$, are given by

$$
K=\frac{E}{3(1-2 v)}
$$

and

$$
\dot{\mathrm{p}}=\mathrm{K} \varepsilon_{\mathrm{ii}} .
$$




\subsection{Post-Processing}

\subsubsection{LS-TAURUS}

LS-TAURUS is the post processor for DYNA3D, NIKE3D, and TOPAZ3D. It reads the binary files written by these packages and plots contours, time histories, deformed shapes etc., Various nodal data like acceleration, velocity, displacement and elemental data like the stress and strain can be plotted. Also the strain energies, internal energies, and kinetic energies for each material can be plotted.

The software is divided into three phases. The first phase can give graphic information like the stress contours, deformation, and displacement in various modes like the shaded image or the line image etc. The second phase is for time history plotting. It can plot various nodal and elemental data. The displacement, velocity, and acceleration can be plotted for nodes against time and the elemental stress and strains can be plotted against time. Also material energies can be plotted individually for different materials. The difference between the values for each nodal component for two nodes can also plotted. The third phase called the ASCII plotting phase can read the ASCII data files written by the DYNA3D code. It can read the ASCII file and plot global data, nodal data, elemental data, rigid wall forces, cross section forces, and the interface forces. Post processing can be done in parallel to the simulation run. While the solution is going on in LS-DYNA3D, the results obtained before the completed time state can be post processed. The file organization for LS-TAURUS is as shown in Figure 4.12. 


\section{File Organization}

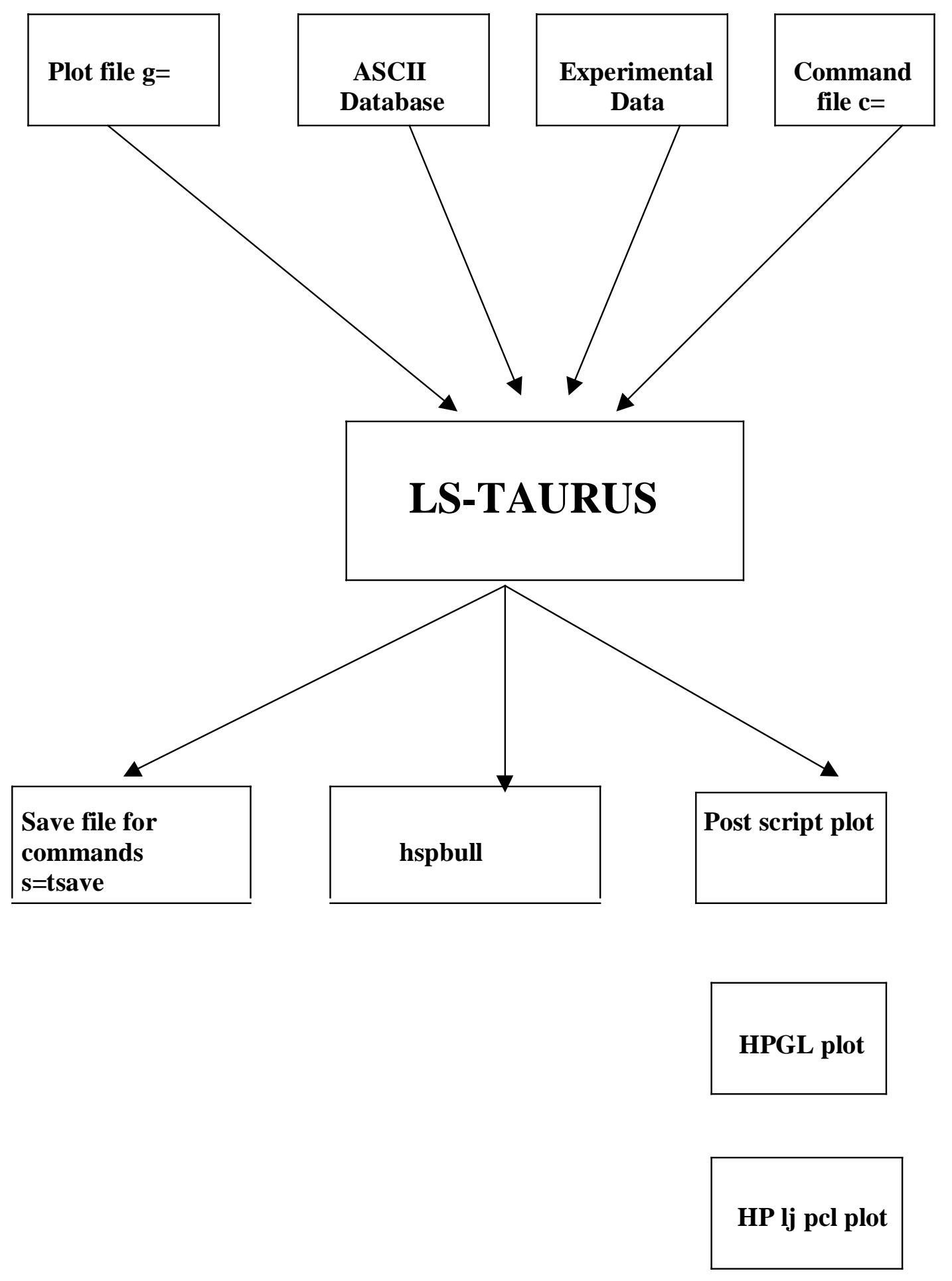

Fig 4.12 File Organization for LS-TAURUS. 


\section{CHAPTER 5 - RESULTS AND DISCUSSION}

\subsection{INTRODUCTION}

The results of the crashworthiness analysis of the school bus are illustrated in this chapter. The results include deformation and time history plots for displacement, velocity, acceleration, and energy. The post processing was done in LS-TAURUS, which can read the binary and the ASCII files written as output from LS-DYNA3D. The results can be classified into two types: the macroscopic results like the global values of energy, velocity, and acceleration and the microscopic information like the displacement, velocity, and acceleration. The entire time of the crash event is divided into 100 time states for the ease of post processing, and each state has all the output information at a specific time of the event. The numerical time step is calculated as illustrated in Section 3.4. The numerical time step is the time taken by the software to move the object from a

position $\mathrm{x}^{\mathrm{n}}$ to a position $\mathrm{x}^{\mathrm{n}+1}$ while the solution is going on. The plot state time step is the time at which the microscopic and the macroscopic output are written by the software. Shading and wire frame options, animation options, fringe and contour plots, and vector displays were used to study and compare the deformation between different components of the bus model at different time states. Two types of crash analyses were performed on the school bus model.

- A full frontal rigid wall impact to simulate impact against a wall.

- An offset frontal rigid wall impact to simulate impact against a corner wall. 
For each impact, a single point integration and a higher order integration was performed to study the effects of hourglassing on the results.

The finite element model in Figure 5.1 shows the full bus model with the coordinate systems used for the analysis. The coordinate system will aid in understanding the following plots. The $\mathrm{X}$ axis is perpendicular to the side of the bus, the $\mathrm{Y}$ axis is perpendicular to the roof, and the $\mathrm{Z}$ axis is along the length of the bus. Table 5.1 lists the part identification number for various components that make up the bus.

Table 5.1 Parts and part numbers.

\begin{tabular}{|c|c|c|}
\hline Part ID & Material & Element Type \\
\hline 1 & Frame & Thin shell \\
\hline 2 & Front axle & Solid \\
\hline 3 & Tires & Solid \\
\hline 4 & Rear axle & Thin shell \\
\hline 5 & Gear box & Solid \\
\hline 6 & Springs & Solid \\
\hline 7 & Radiator & Thin shell \\
\hline 8 & Stub axle & Solid \\
\hline 9 & Fuel tank & Thin shell \\
\hline 10 & Engine & Solid \\
\hline 11 & Body skin & Thin shell \\
\hline 12 & Rib structure & Solid \\
\hline 13 & Radiator Mount & Solid \\
\hline 14 & Bumper & Thin shell \\
\hline 15 & Rear axle shaft & Solid \\
\hline 16 & Connecting struts & Thin shell \\
\hline 17 & Hood & Thin shell \\
\hline 18 & Fire wall & Solid \\
\hline
\end{tabular}




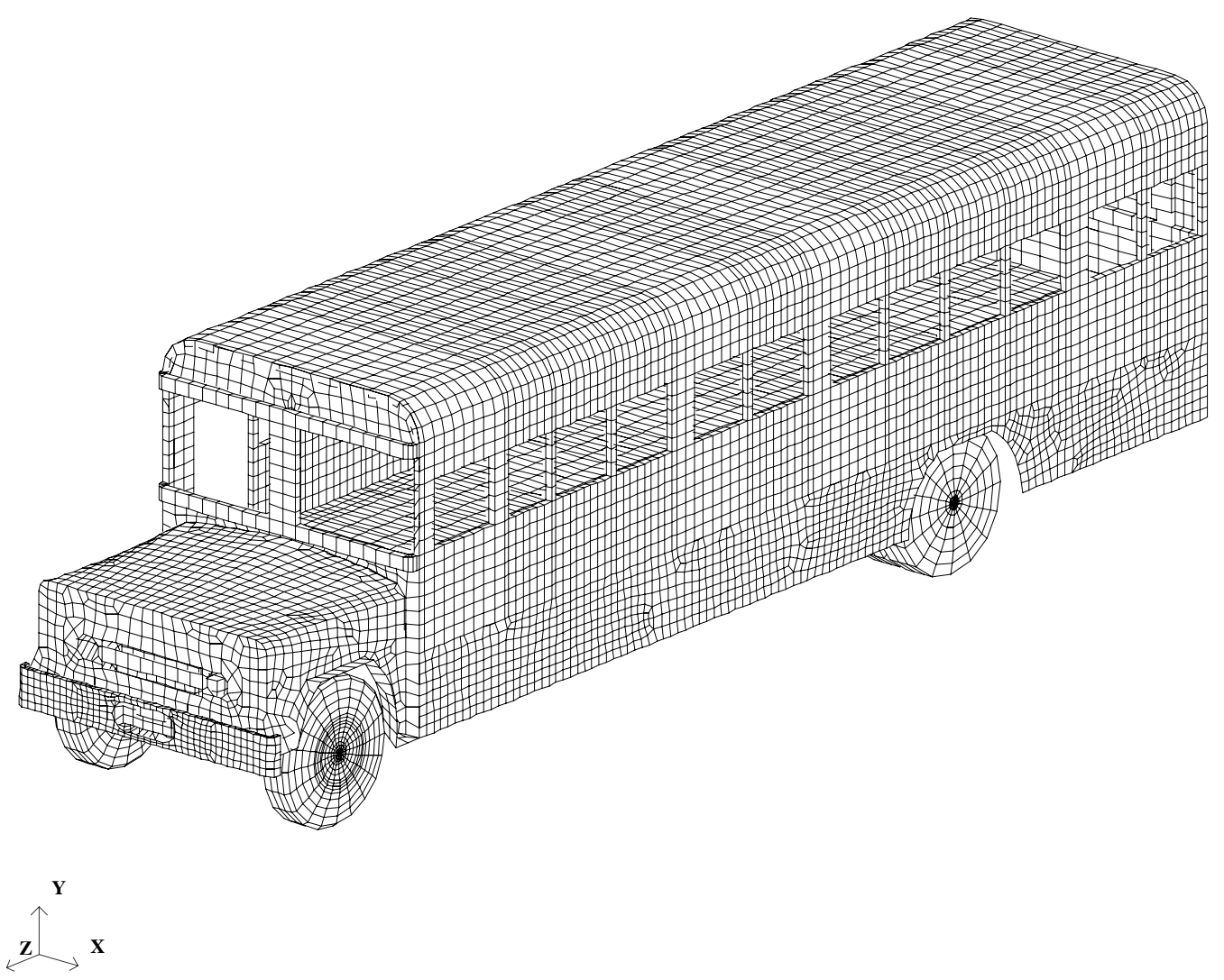

Fig 5.1 Full finite element model of the school bus with coordinate system.

The initial velocity of $56 \mathrm{~km} / \mathrm{hr}$ was given in the positive $\mathrm{Z}$ direction to all the nodes of the finite element model for the frontal impacts. The rigid wall was defined with its vector in the negative $\mathrm{Z}$ direction for the frontal impacts. According to the vehicle coordinate system, the pitch of the vehicle is the rotation about the $\mathrm{X}$ axis, the roll of the vehicle is the rotation about the $\mathrm{Z}$ axis, and the yaw is the rotation about the $\mathrm{Y}$ axis. These terms are used to define the behavior of the vehicle in different crash scenarios. 


\subsection{FULL FRONTAL IMPACT WITH BELYTSCHKO -TSAY THIN SHELL ELEMENTS}

The front view of the bus subjected to a full frontal impact is shown in Figure 5.2. The rigid wall is defined in front of the bus with its vector pointing in the opposite direction of the velocity vector. The head of the normal vector of the rigid wall is 0.1 meters away from the bumper, which means that the bus will hit the rigid wall after travelling a distance of 0.1 meters at a initial velocity of $56 \mathrm{~km} / \mathrm{hr}$; this corresponds to $15.55 \mathrm{~m} / \mathrm{sec}$. Figures 5.3 to 5.30 illustrate the results for the full frontal impact.

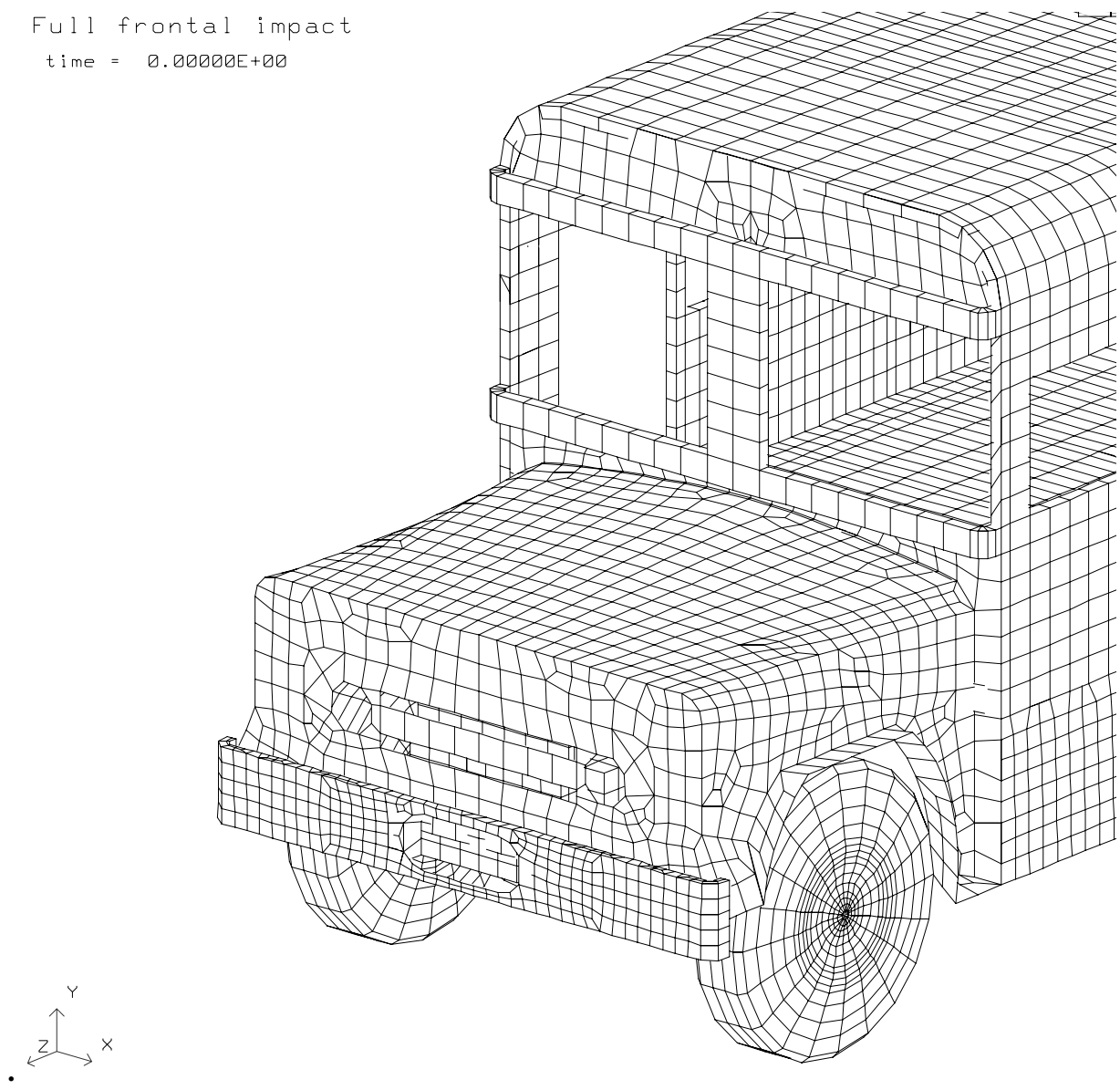

Fig 5.2 Front view at time $=0 \mathrm{msec}$ of the bus subjected to a full frontal impact at a velocity of $56 \mathrm{~km} / \mathrm{hr}$.

Figure 5.3 shows the front view of the vehicle at time $=9 \mathrm{msec}$. The bus has hit the rigid wall and the deformation can be seen on the bumper. The hood has just reached the 
rigid wall and the deformation is yet to occur on the hood. The kinetic energy of the vehicle lessens and is absorbed as the internal energy of the parts that hit the rigid wall, which can be seen from Figure 5.29. The rigid wall force reaches $8 \mathrm{MN}$ before $5 \mathrm{msec}$ which shows that the bumper has hit the rigid wall. The majority of the internal energy of the vehicle at this time is absorbed by the frame as seen in Figure 5.28.

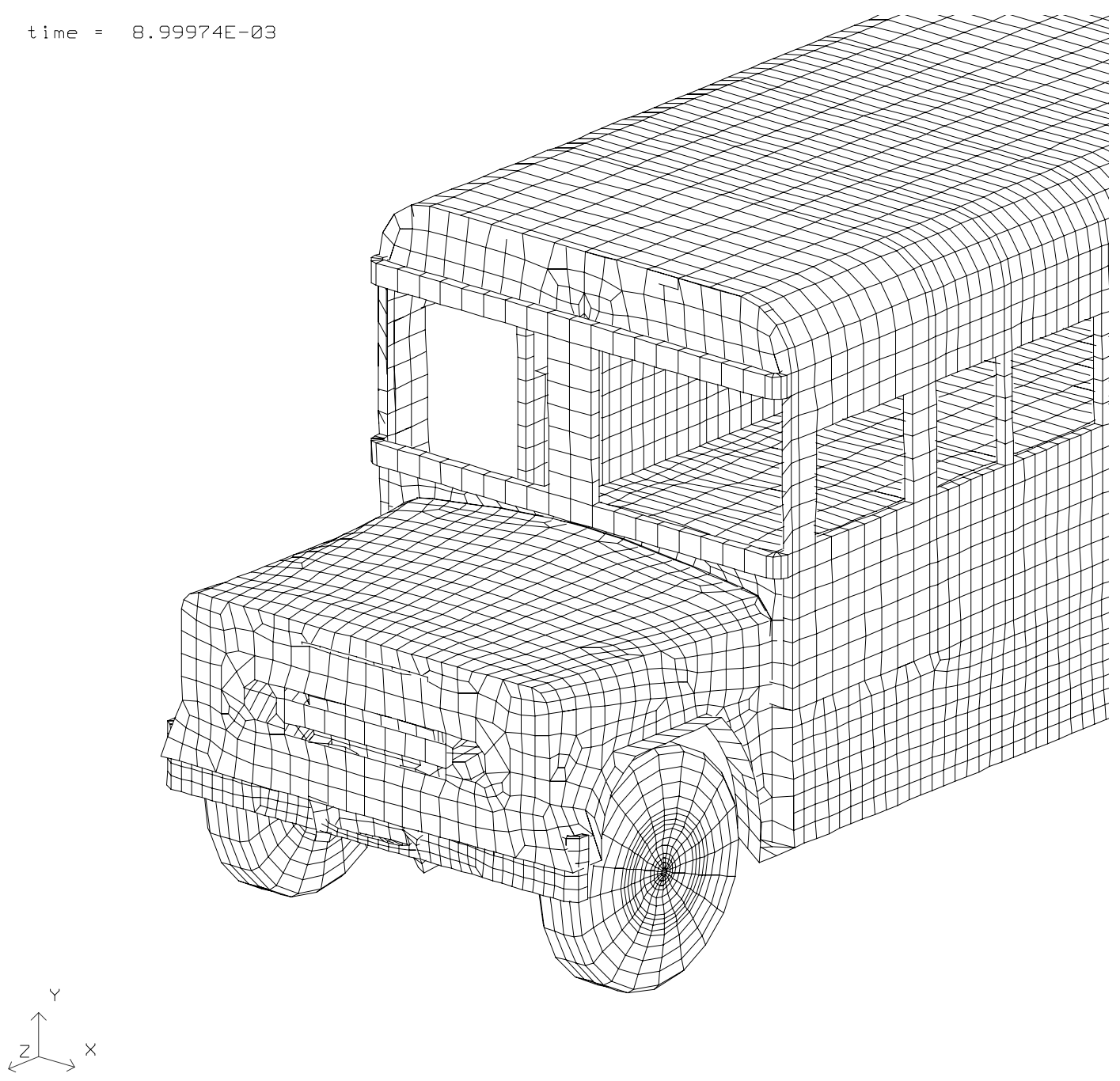

Fig 5.3 Front view at time $=9 \mathrm{msec}$ of the bus subjected to a full frontal impact at a velocity of $56 \mathrm{~km} / \mathrm{hr}$.

The front view of the bus at time $=18 \mathrm{msec}$ is shown in Figure 5.4. The hood has impacted the rigid wall by this time. The deformation to the hood can be seen in Figure 
5.4. The kinetic energy of the vehicle continues to decrease rapidly and the internal energy increases, as can be seen from Figure 5.29. The relative displacement between the front tires and the frame is compared in Figure 5.15, which shows that the front tires move in the positive $\mathrm{Z}$ direction at a higher rate than the frame, showing that the frame is compressed as it hits the rigid wall and the spring undergoes a rigid body displacement. The relative displacement increases in the negative $\mathrm{Z}$ direction as seen from the graph in Figure 5.15.

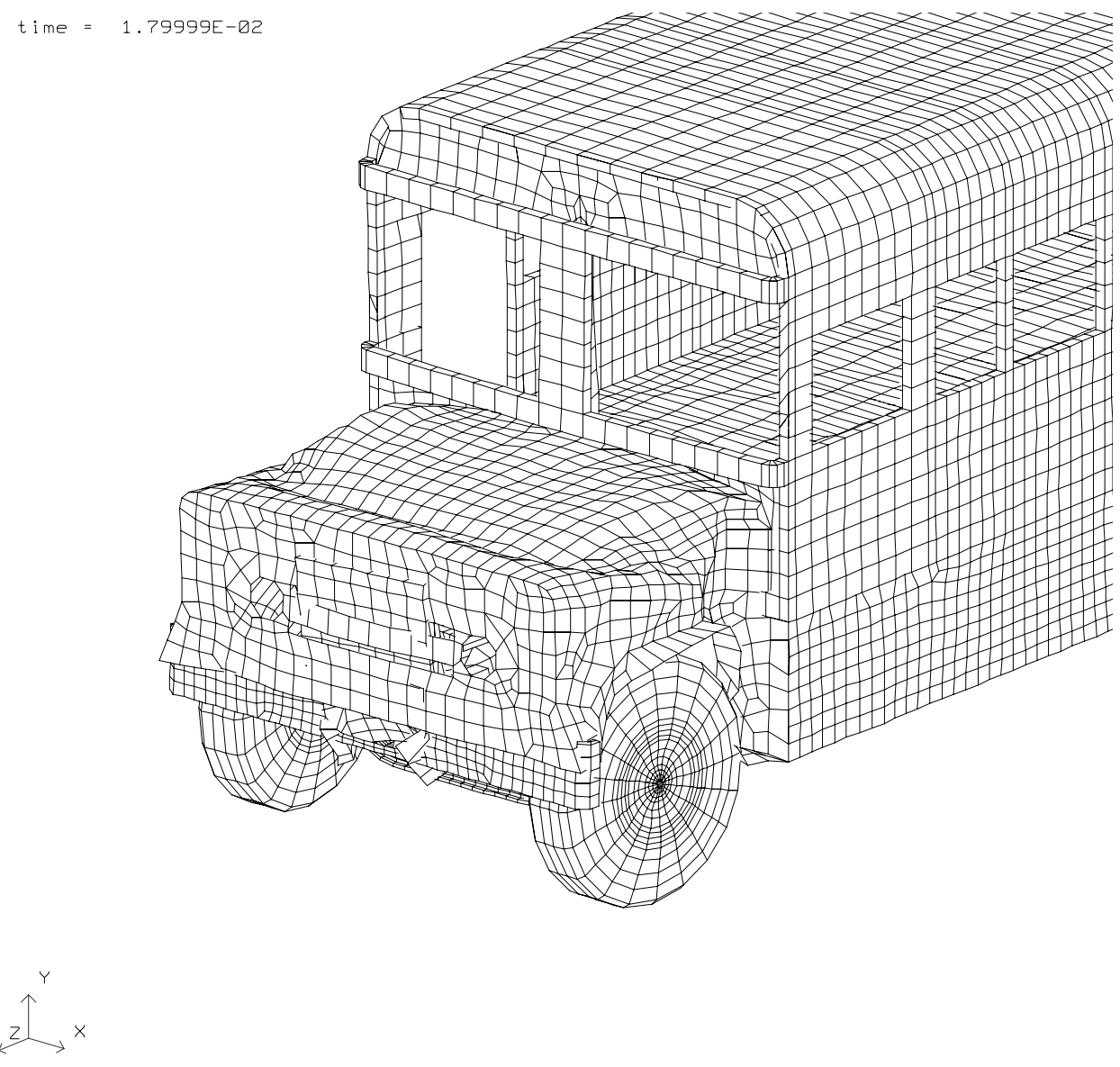

Fig 5.4 Front view at time $=18 \mathrm{msec}$ of the bus subjected to a full frontal impact at a velocity of $56 \mathrm{~km} / \mathrm{hr}$.

The front end of the vehicle at time $=27 \mathrm{msec}$ is shown in Figure 5.5. The front cross member of the frame and the side members also hit the rigid wall and energy is 
absorbed by the frame. The internal energy absorbed by the frame and the engine reaches a maximum at around $30 \mathrm{msec}$ and then drops down, which can be seen in Figure 5.28. The VonMises yield stress in the firewall is less than the yield stress of steel and hence no plastic deformation is on the fire wall. The total displacement on the firewall is $0.3 \mathrm{~m}$, which does not protrude into the occupant area.

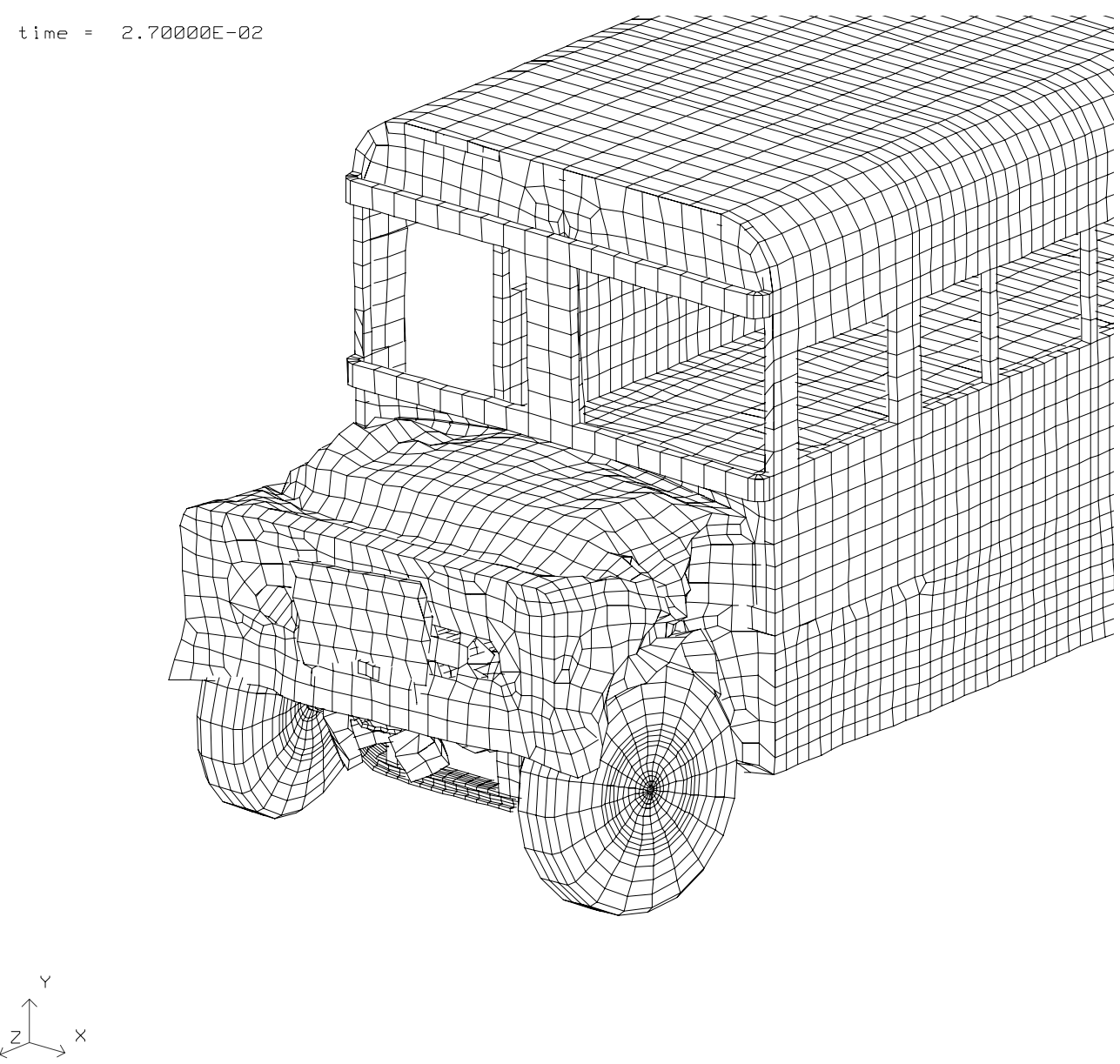

Fig 5.5 Front view at time $=27 \mathrm{msec}$ of the bus subjected to a full frontal impact at a velocity of $56 \mathrm{~km} / \mathrm{hr}$.

The front view of the vehicle at time $=36 \mathrm{msec}$ is shown in the Figure 5.6. The front wheels start to toe in at this time. The $\mathrm{Z}$ relative displacement between the nodes in the spring and the nodes in the frame increases as seen in Figure 5.15, which shows that the 
spring allows a longitudinal displacement in the $\mathrm{Z}$ direction for the tires. The $\mathrm{Z}$ velocity of the nodes in the center of the firewall is around $11 \mathrm{~m} / \mathrm{sec}$. The total displacement in the firewall is 0.308 meters, which would not cause any intrusion of the front end parts to the occupant area.

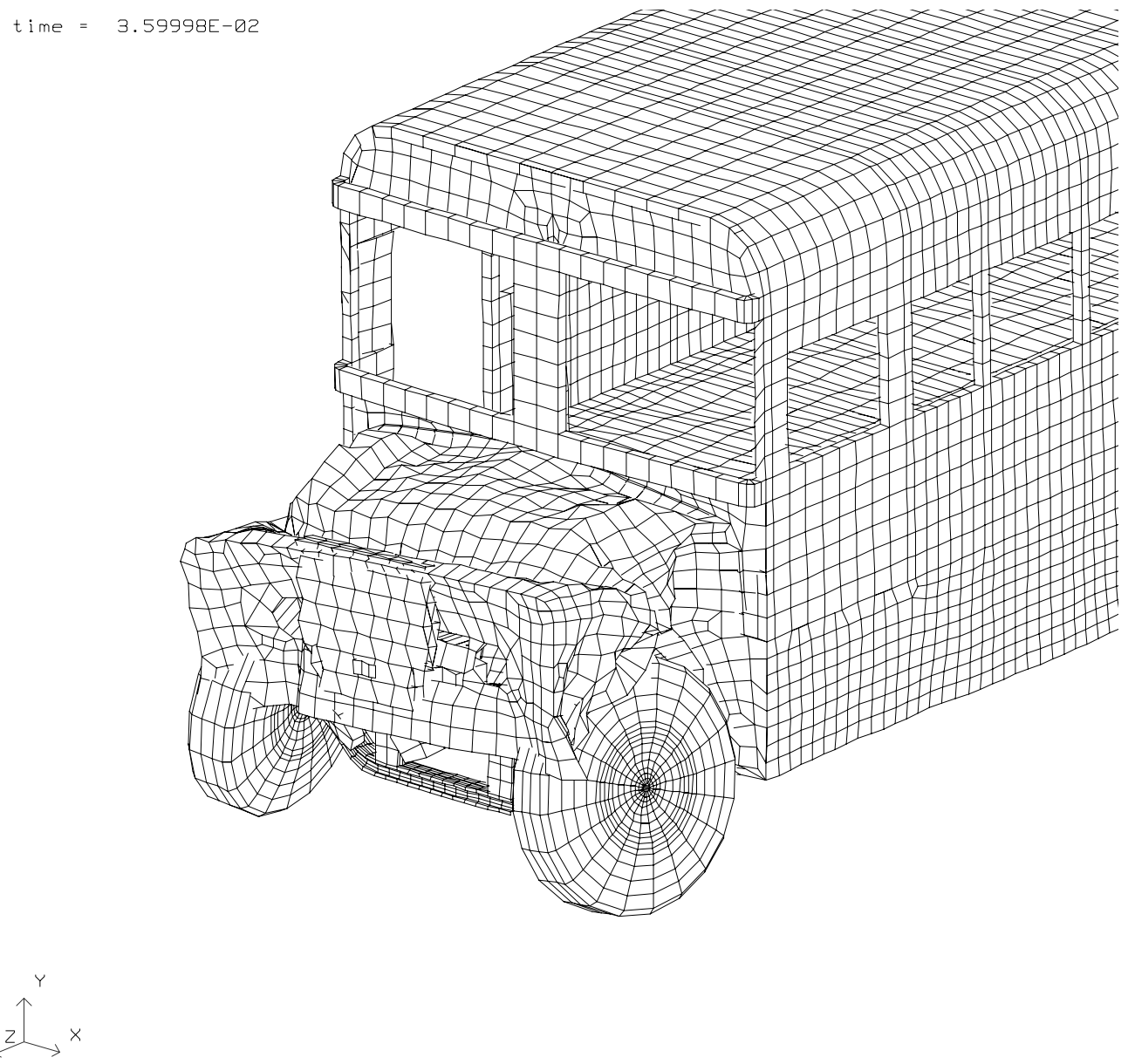

Fig 5.6 Front view at time $=36 \mathrm{msec}$ of the bus subjected to a full frontal impact at a velocity of $56 \mathrm{~km} / \mathrm{hr}$.

The front view of the bus at time $=45 \mathrm{msec}$ is shown in Figure 5.7. The hood buckles at the center line along the $\mathrm{X}$ axis. The front end of the frame also buckles along with the hood. When the Y displacement between the nodes in the front tire and the rear tire are compared as seen in Figure 5.16, the relative displacement is less up to $45 \mathrm{msec}$ 
and it increases rapidly up to $80 \mathrm{msec}$. This shows that the vehicle is rotating or pitching about the $\mathrm{X}$ axis. There is minimal rotation about the $\mathrm{Y}$ and the $\mathrm{Z}$ axis. The kinetic energy continues to decrease and it is converted as material internal energy as seen in Figure 5.29.

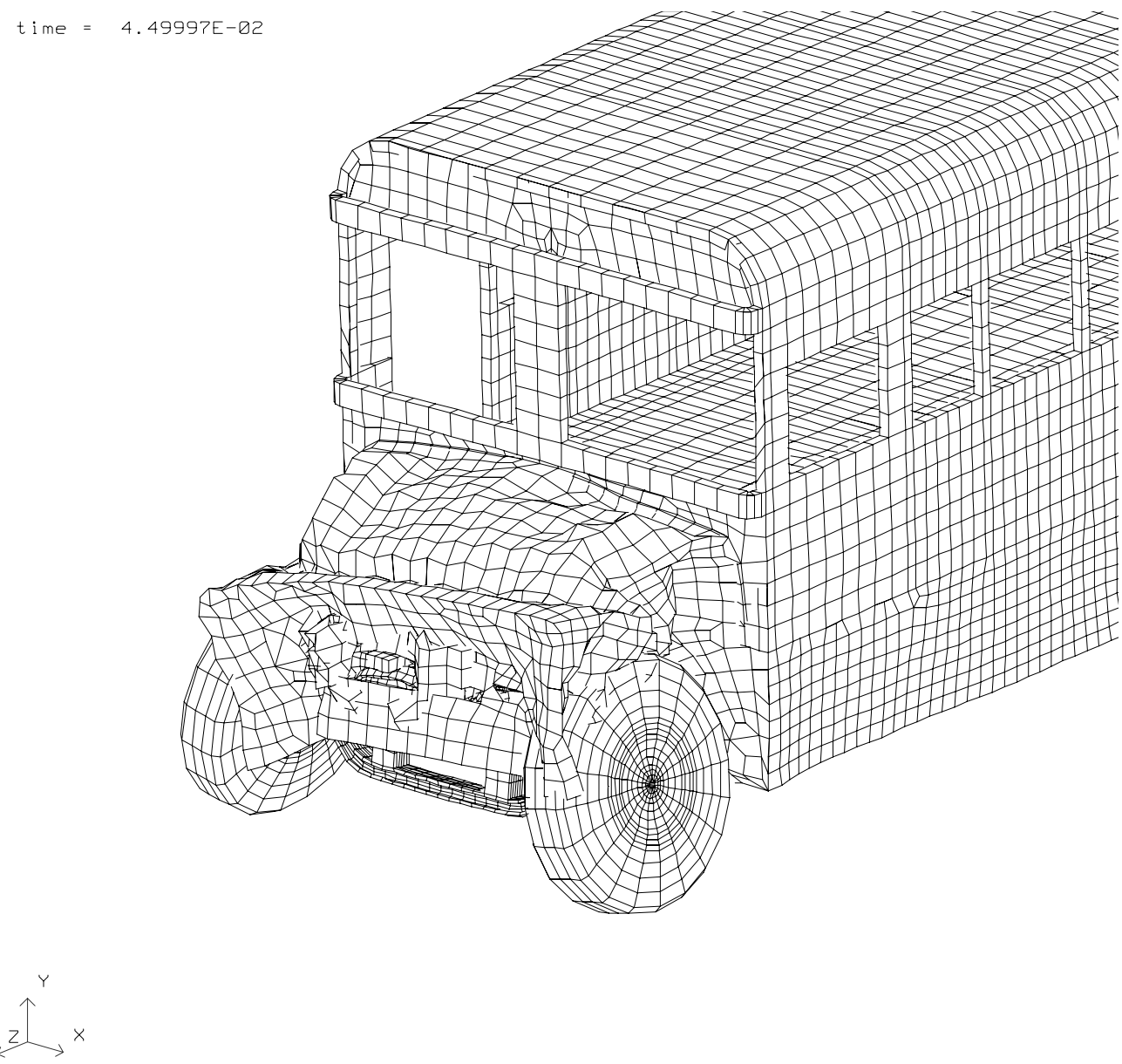

Fig 5.7 Front view at time $=45 \mathrm{msec}$ of the bus subjected to a full frontal impact at a velocity of $56 \mathrm{~km} / \mathrm{hr}$.

The front view of the chassis alone is shown in Figure 5.8 at time $=54$ msec. The deformation in the springs can be seen, which causes the longitudinal movement of the chassis alone from the body. The radiator and the engine also absorb energy and the deformation in the radiator can be seen in the figure. The propeller shaft bends about the 
lateral axis (X axis) and this deformation is exaggerated because the bolts connecting the propeller shaft to the frame were not modeled. The deformation in the front end of the frame is also illustrated. The deflection of the front wheels may also be high because the components like the steering lever and the stub axle that add a resistance to wheel deflection were not modeled.

time $=5.39999 \mathrm{E}-02$
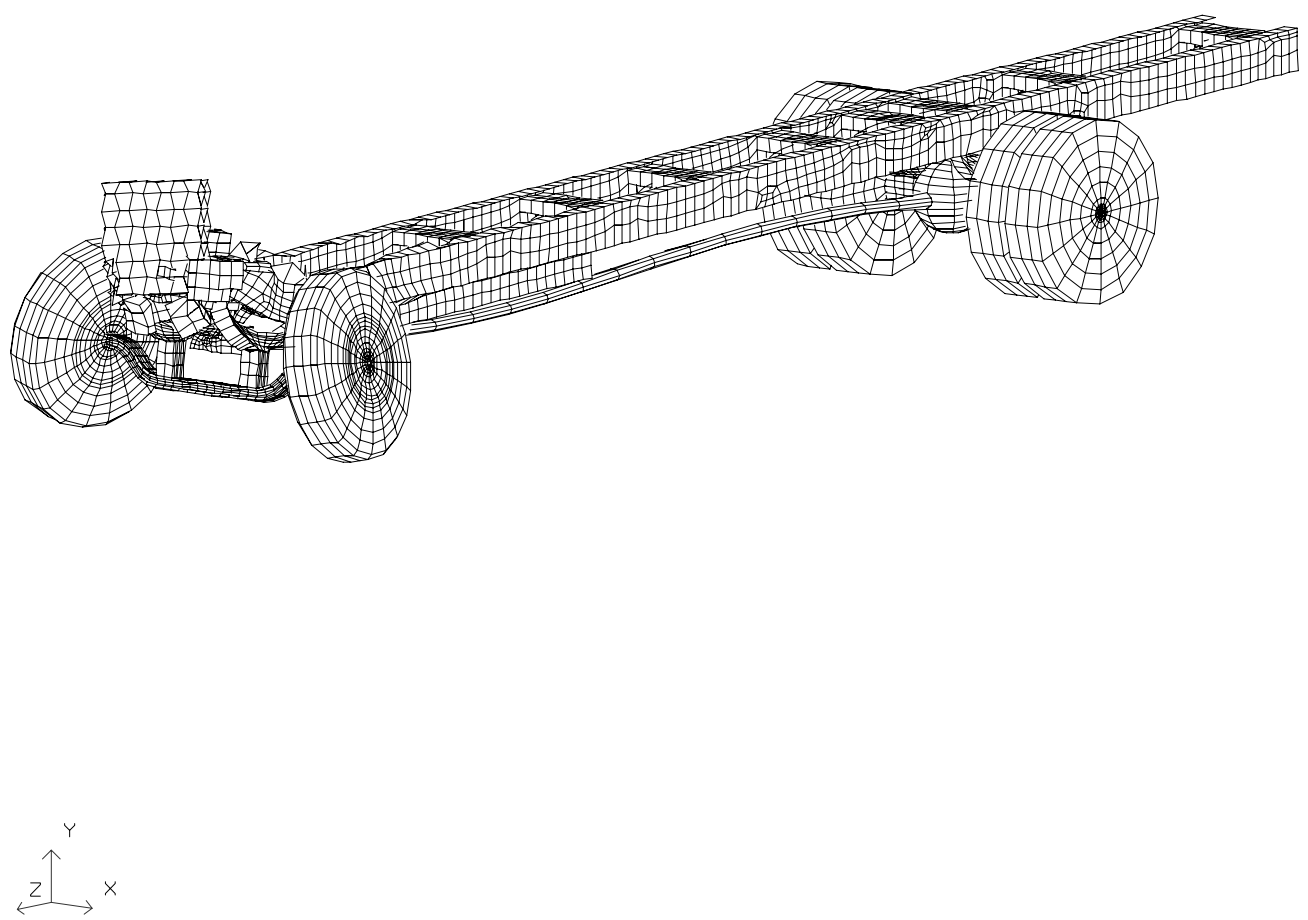

Fig 5.8 Front view at time $=\mathbf{5 4} \mathrm{msec}$ of the bus chassis subjected to a full frontal impact at a velocity of 56 $\mathbf{k m} / \mathbf{h r}$.

Figure 5.9 shows the deformation in the front end of the chassis at time $=72 \mathrm{msec}$. The front end of the frame raises up though the front wheel and the tires move down. The spring seats also deform and yield to the longitudinal movement of the springs as 
illustrated. The maximum stress on the fire wall is equal to the yield stress of steel at this time state and the absolute maximum displacement is 0.51 meters, which would not cause any intrusion into the occupant area. The maximum stress in the deformed area at front end of the frame is higher than the yield stress of the material and it has entered the plastic region.

time $=7.19999 E-02$
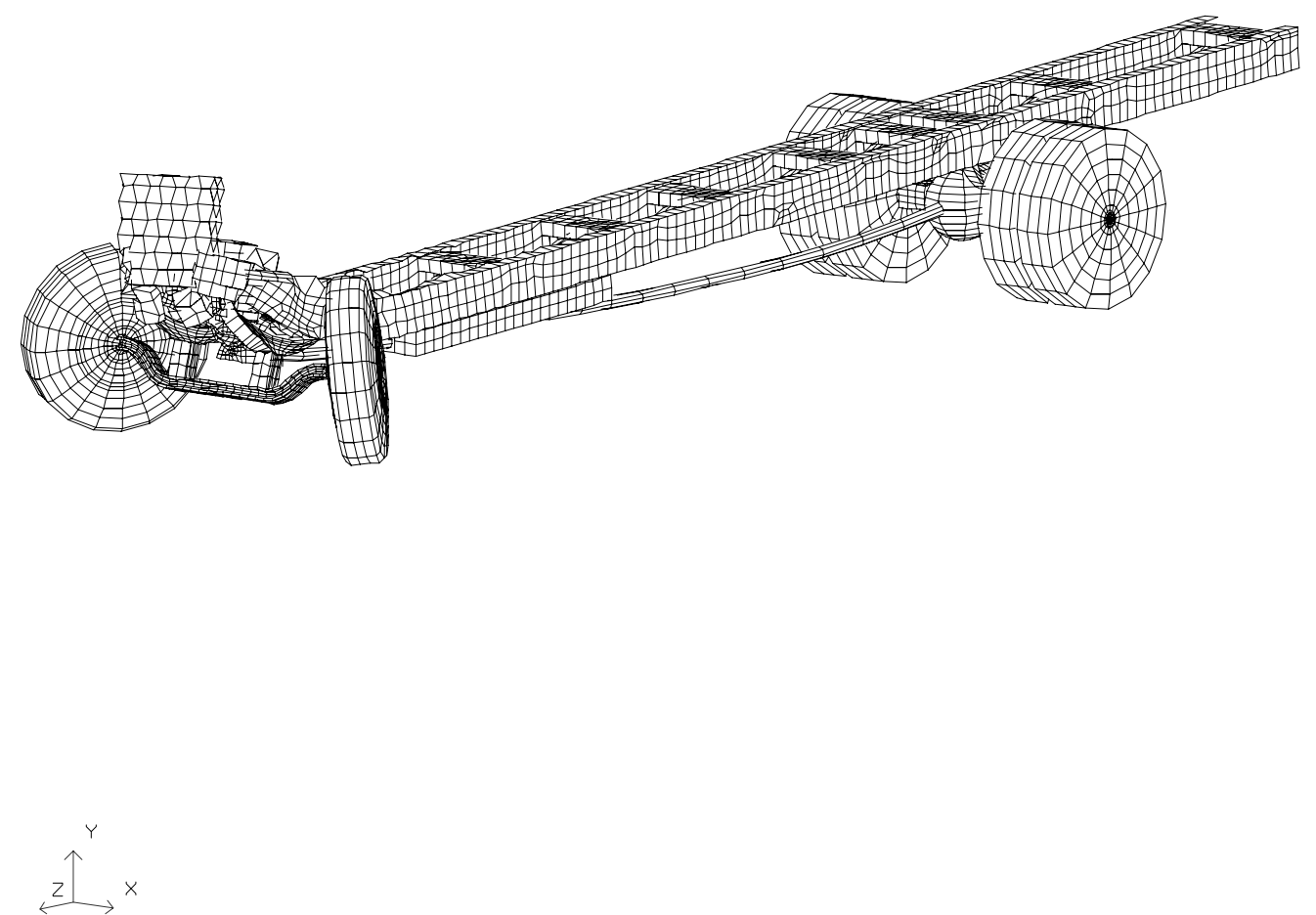

Fig 5.9 Front view at time $=72 \mathrm{msec}$ of the bus chassis subjected to a full frontal impact at a velocity of 56 km/hr.

Figure 5.10 shows the final state of the bus subjected to a full frontal impact at a velocity of $56 \mathrm{~km} / \mathrm{hr}$. The tires and axle are not shown in the picture. The final absolute deformation on the fire wall is $0.55 \mathrm{~m}$, which does not intrude into the occupant area in 
the front end of the vehicle. The body skin on the driver end of the vehicle undergoes a deformation and bulges inside and the maximum absolute deformation is 0.03 meters. The body skin has two layers with a outer and a inner skin and the outer skin does not touch the inner skin.
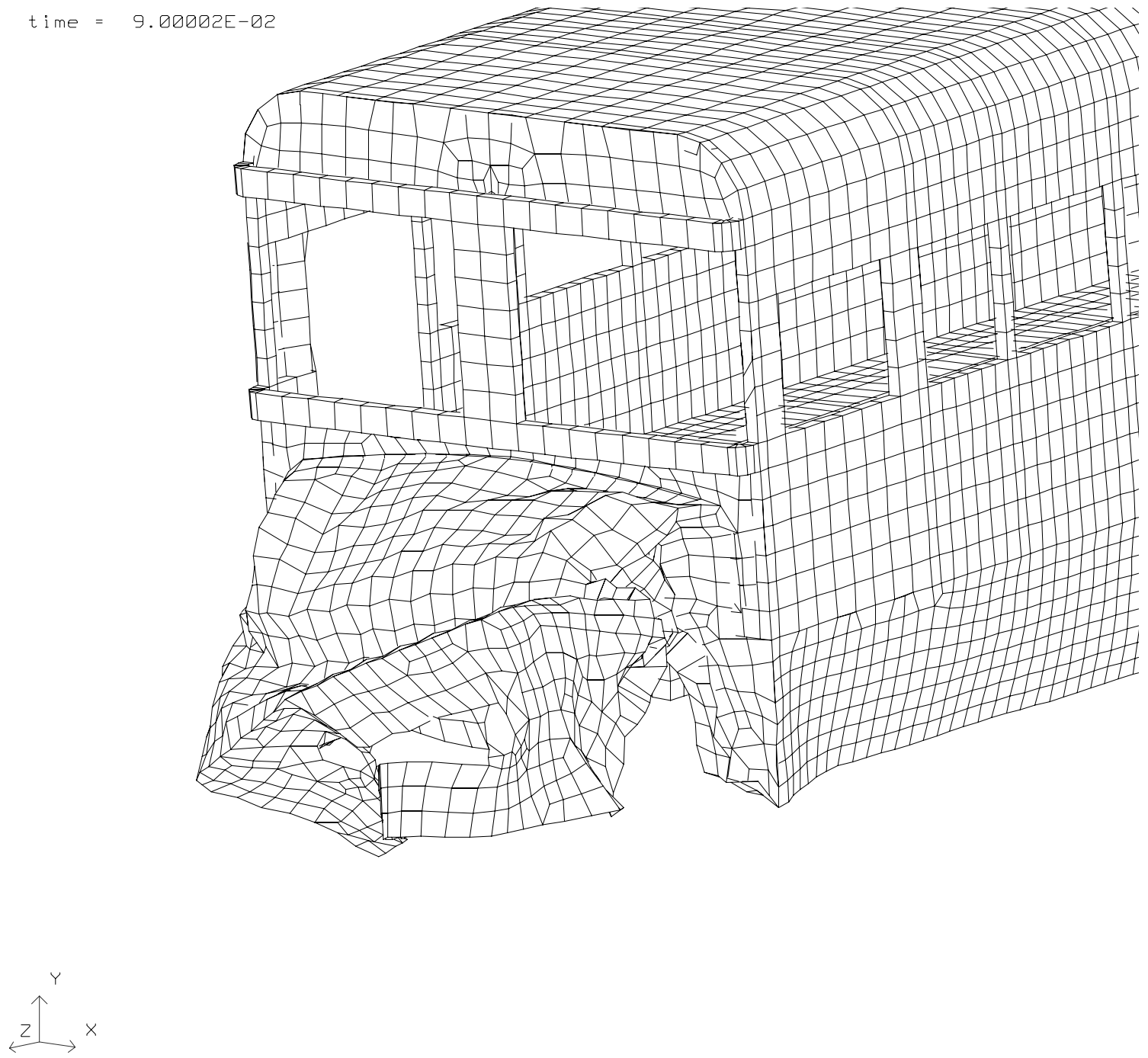

Fig 5.10 Front view at time $=90 \mathrm{msec}$ of the bus subjected to a full frontal impact at a velocity of $56 \mathrm{~km} / \mathrm{hr}$.

Figure 5.11 shows the side view of the bus subjected to a full frontal impact at a velocity of $56 \mathrm{~km} / \mathrm{hr}$ at time $=90 \mathrm{msec}$. The front end of the vehicle moves down in the $\mathrm{Y}$ 
axis with its rear end pivoted and the vehicle rotates about the $\mathrm{X}$ axis. This is called the pitching of the vehicle and this phenomenon might cause the occupants of the bus to be thrown out of their seats. The top view of the vehicle subjected to a full frontal impact is shown in Figure 5.12 at time $=90 \mathrm{msec}$. The bulging of the body skin at the rear end of the vehicle can be seen from this figure. The maximum displacement of the skin in the $\mathrm{X}$ direction at the rear end is 0.158 meters. Figure 5.13 shows the side view of the frame which shows the pitching of the vehicle. The front end of the frame slides and rises in the positive $\mathrm{Y}$ direction after it hits the rigid wall as illustrated.

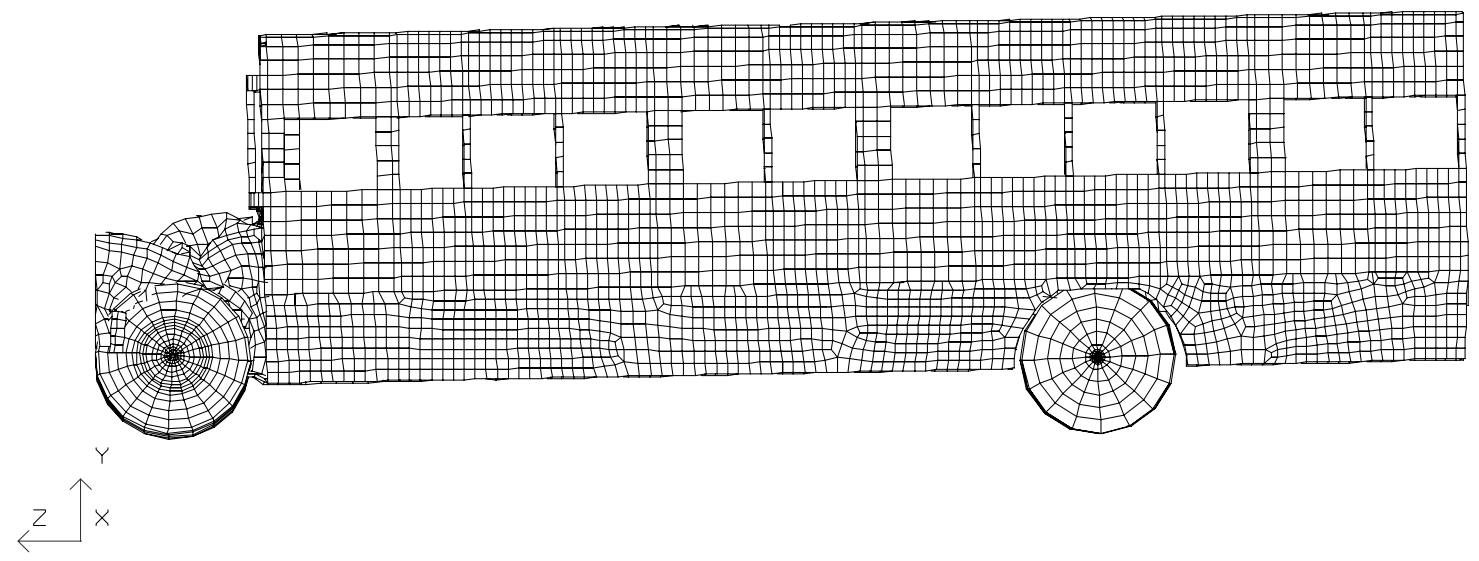

Fig 5.11 Figure showing the side view of the bus at time $=\mathbf{4 5} \mathbf{m s e c}$ for full frontal impa7ct. 

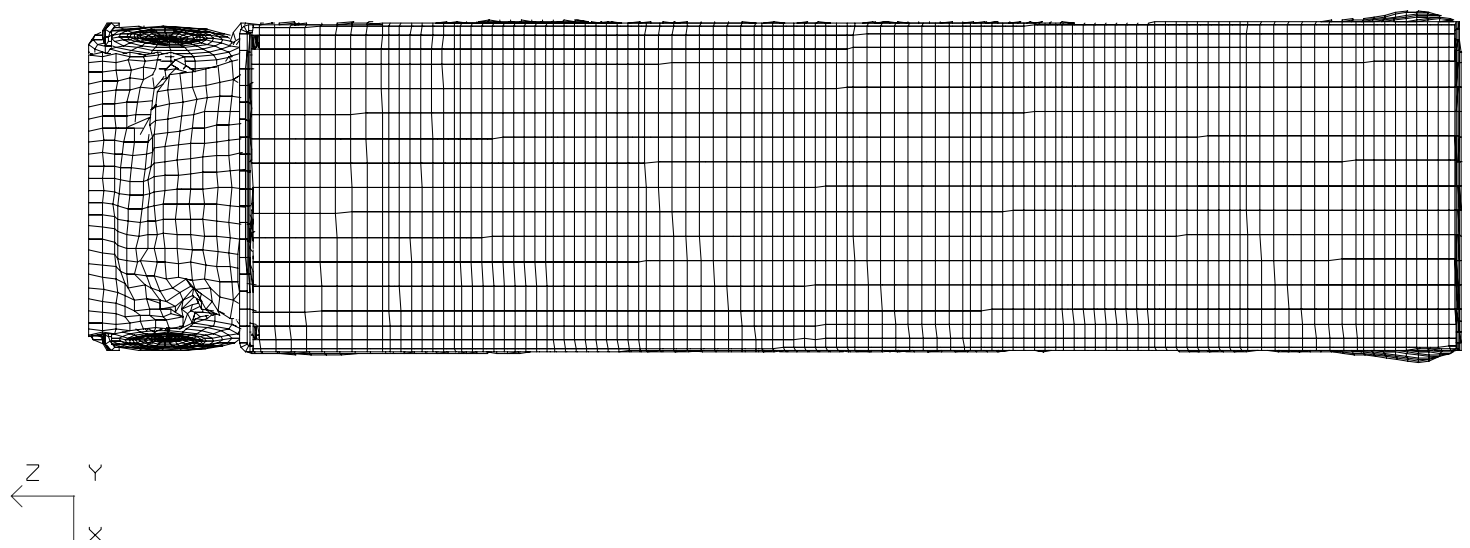

Fig 5.12 Figure showing the top view of the bus at time $=\mathbf{4 5} \mathbf{m s e c}$ for full frontal impact.
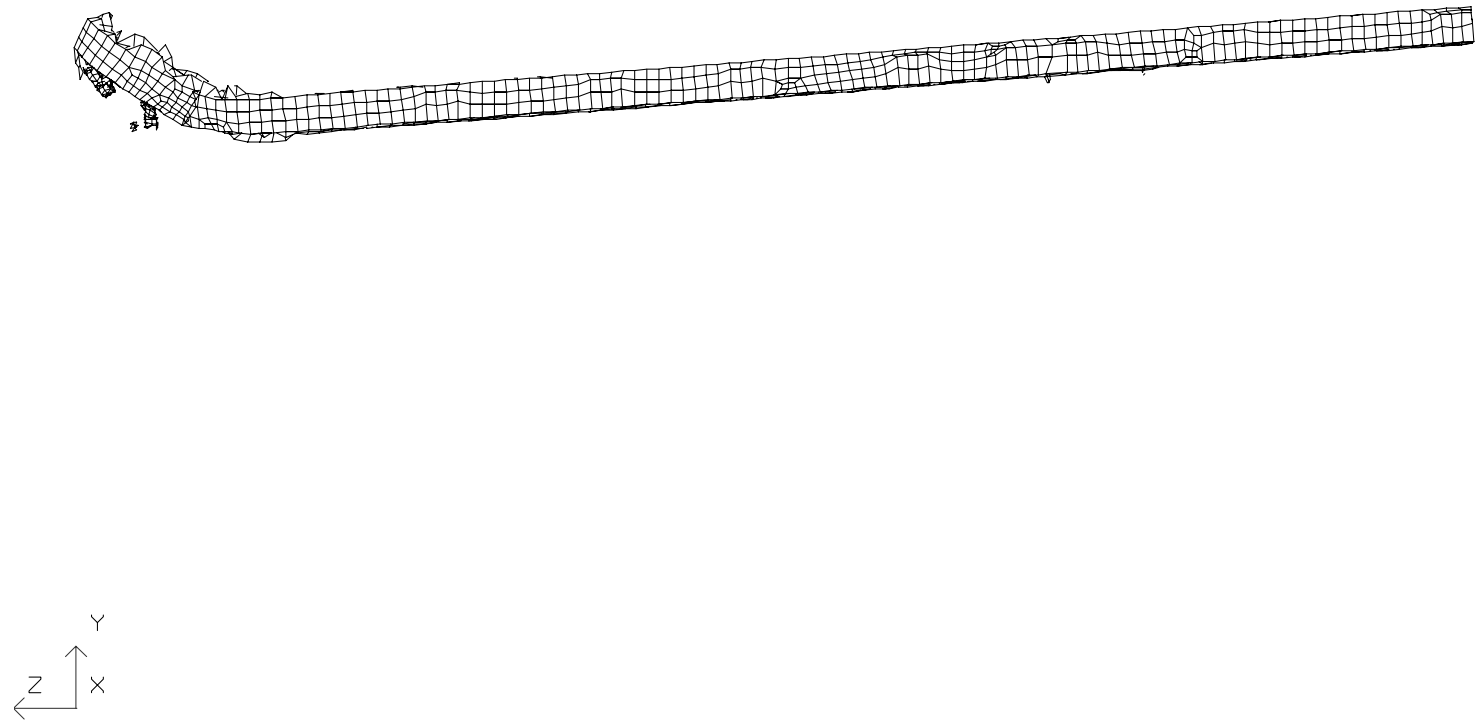

Fig 5.13 Figure showing the deformation of the frame at time $=90 \mathrm{msec}$ for a full frontal impact.

Figure 5.14 shows the VonMises stress plot in the front end of the frame. The maximum stress in the frame is higher than the yield stress of the material of the frame, which shows that the deformation is plastic and the material has yielded. The yield 
strength of the material of the frame can be seen in Appendix C. The front cross member and the two engine mounting cross members have also failed at this time state. The front end of the frame moves in the $\mathrm{Y}$ direction as seen in Figure 5.13, and the stress contours were plotted for the middle surface of the shell elements that makeup the frame.

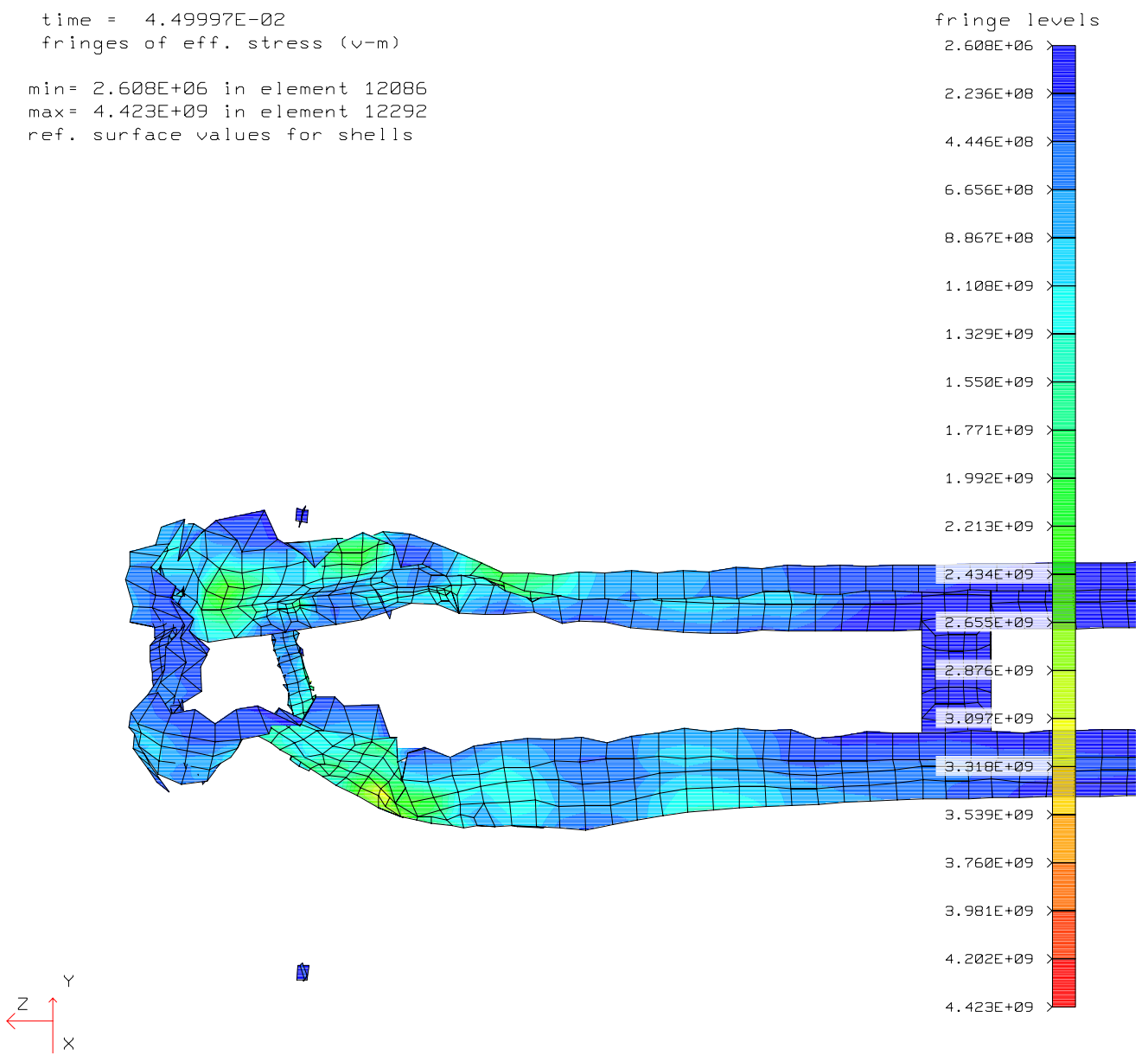

Fig 5.14 Figure showing the VonMises stress plot in the front end of the frame at time $=45 \mathrm{msec}$.

Figure 5.15 shows the relative displacement between nodes in the front end of the frame and the spring. When the frame hits the rigid wall, the leaf spring yields in the longitudinal direction and prevents the deformation of the frame in the initial stages of impact. The graph shows the relative displacement continuously increasing after $15 \mathrm{msec}$ 
which shows that the spring continues to move forward in the $\mathrm{Z}$ direction even if the frame hits the rigid wall. This leads to the longitudinal movement of the front axle and the spring seats in the $\mathrm{Z}$ direction. Figure 5.16 shows the relative displacement between the nodes in the front tire and the rear tire. The relative displacement first increases in the positive direction, showing the front axle first rises from its position up to $25 \mathrm{msec}$. Then the relative displacement continuously decreases to become negative, which shows that the front axle moves in the negative $\mathrm{Z}$ direction thereafter. This shows that the pitching of the vehicle happens only after $25 \mathrm{msec}$.

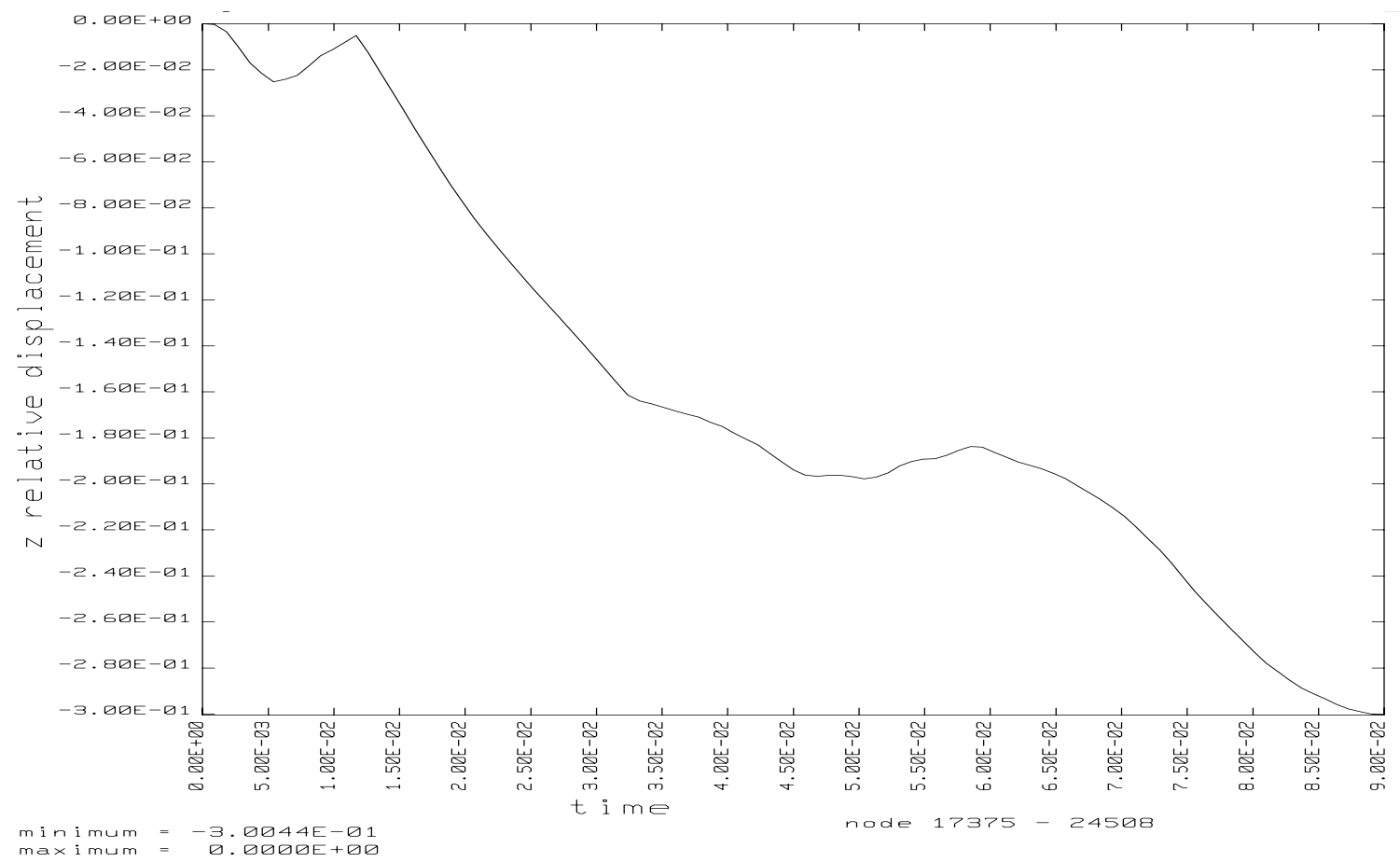

Fig 5.15 Relative displacement between the frame and the front tire. 


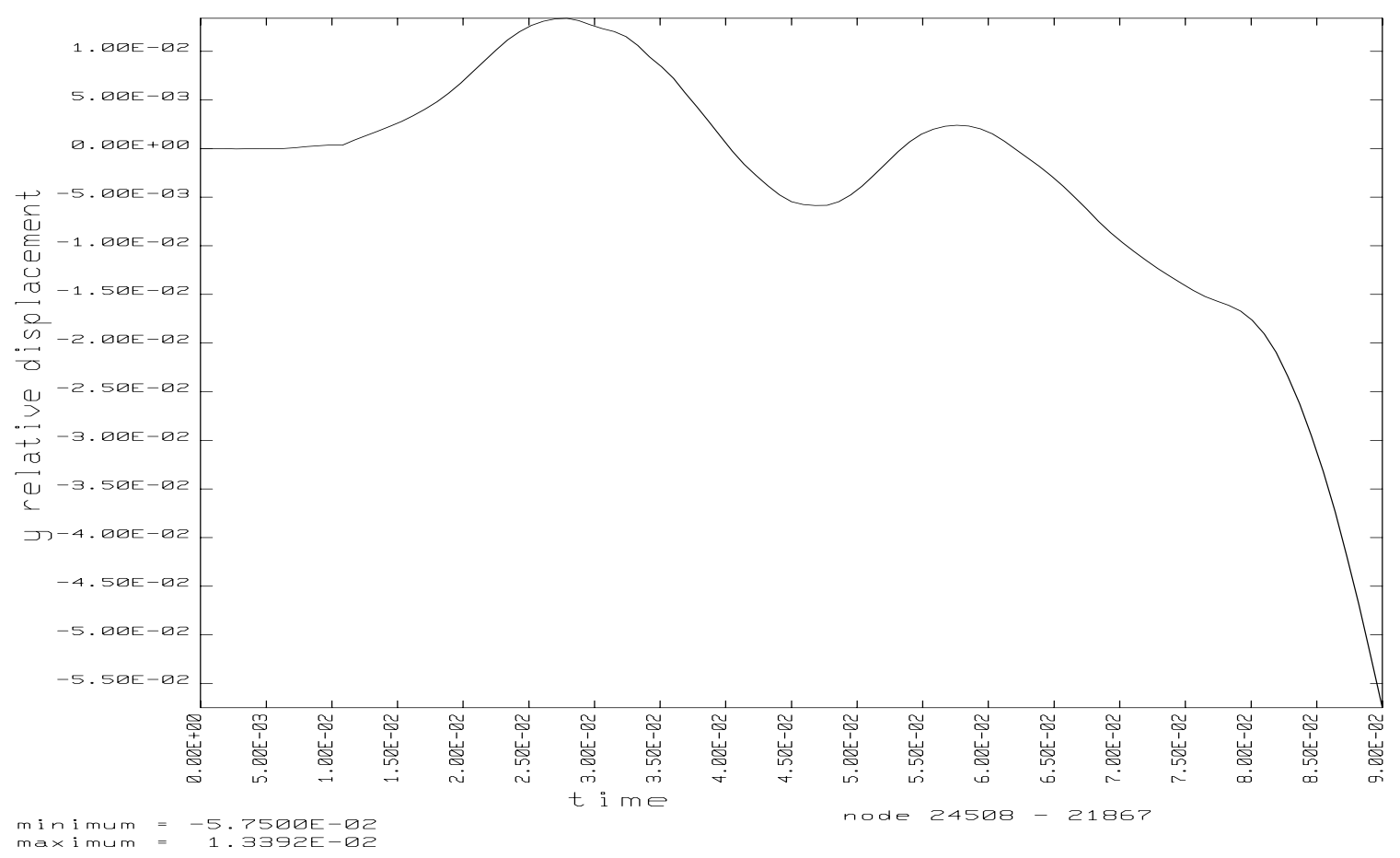

Fig 5.16 Relative displacement between the front tire and the rear tire (pitching).

Figures 5.17, 5.18, and 5.19 show the displacement of the nodes in the front, middle, and the rear of the vehicle. The front of the vehicle is the position along the $\mathrm{X}$ axis from the driver seat to the doorway in the front, the middle of the vehicle is the center position between the rear end and the driver seat end of the vehicle (along the $\mathrm{X}$ axis), and the rear of the vehicle is the position at the rear end of the vehicle (along the $\mathrm{X}$ axis), where the indicators are positioned. The nodes were chosen to represent the left skin, center floor and the right skin for all three portions of the bus. These points were selected to determine if there was any difference in the dynamics between the front, center, and rear of the passenger seating areas for the frontal collision. From Figures 5.17, 5.18, and 5.19, it can be inferred that the maximum displacement in the $\mathrm{Z}$ direction for the nodes in the front, middle, and the rear of the vehicle remains almost the same. This 
shows that there is no protrusion of any part of the vehicle in to the occupant area, and there is only a small deformation to the rib structure and the body skin.

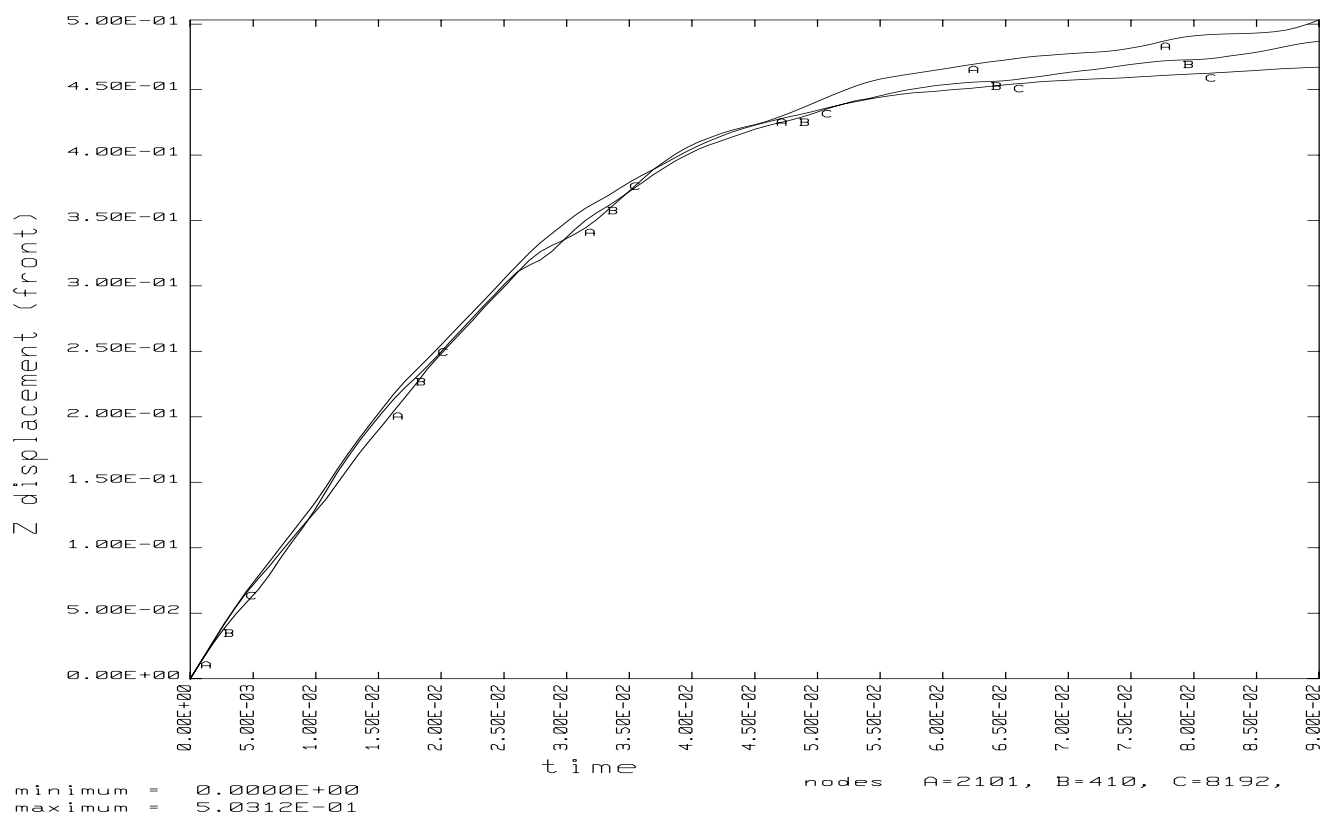

Fig 5.17 $\mathrm{Z}$ displacement for nodes in the front of vehicle.

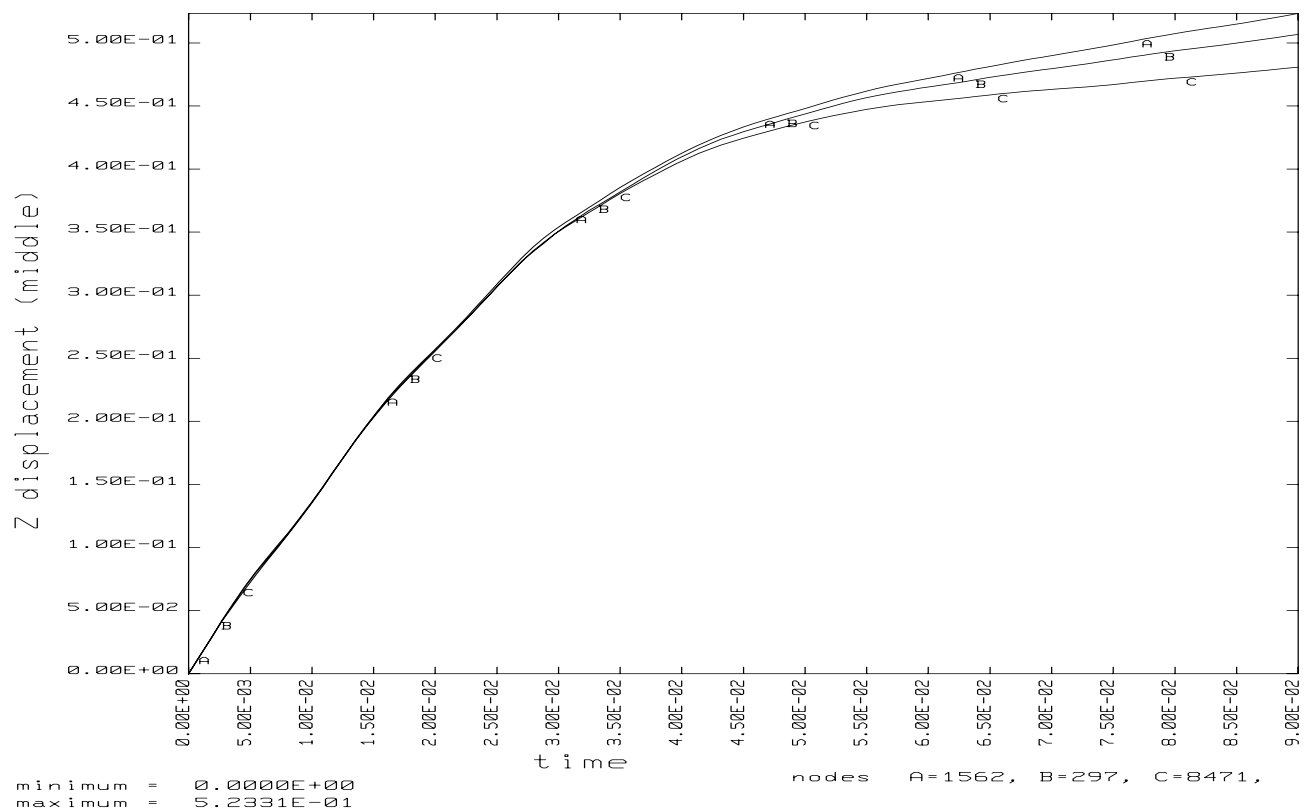

Fig 5.18 $\mathrm{Z}$ displacement for nodes in the middle of vehicle. 


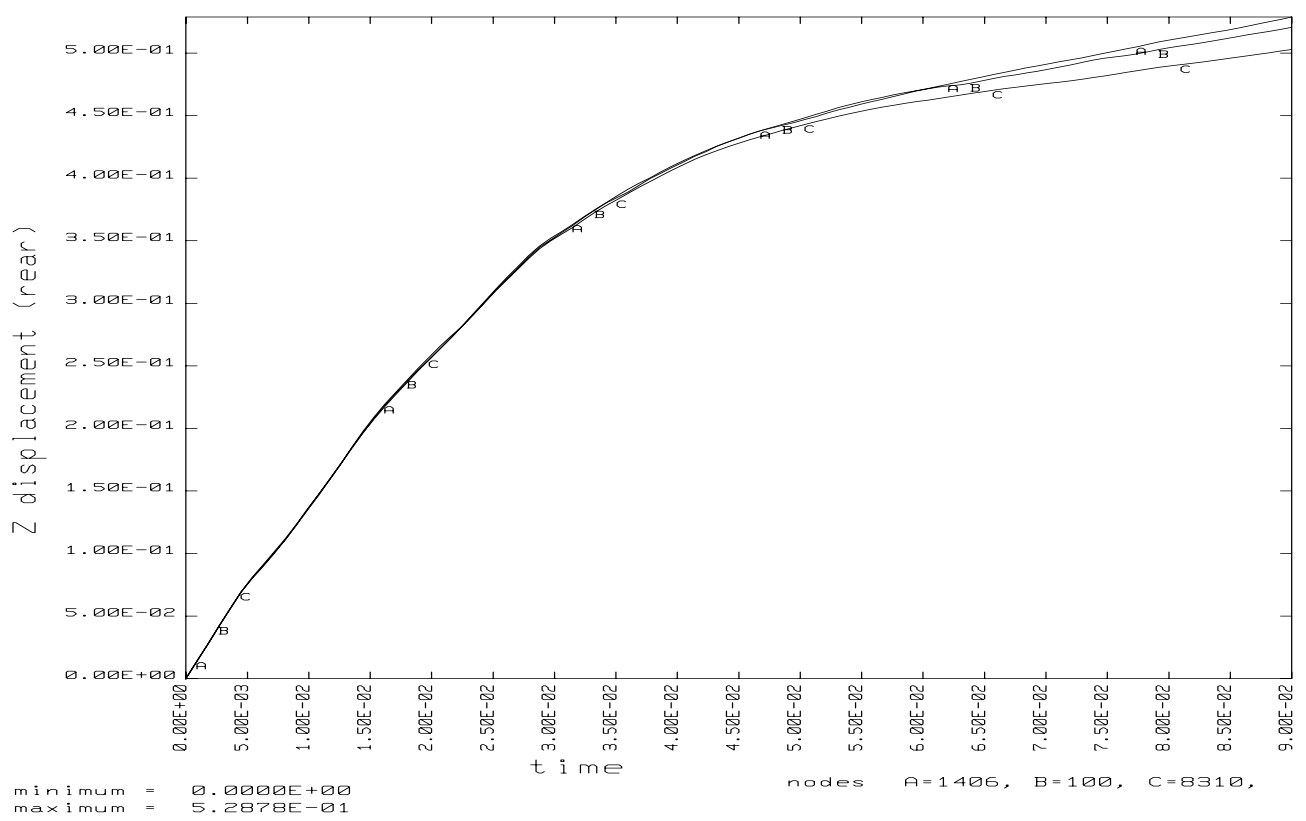

Fig 5.19 $\mathrm{Z}$ displacement for nodes in the rear of vehicle.

Figures 5.20, 5.21, and 5.22 show the velocity of the nodes in the front, middle, and the rear of the vehicle. From the graphs, it can be inferred that the velocity of the nodes in the front, middle, and the rear of the vehicle follow the same pattern. This shows that the kinetic energy of the vehicle continues to decrease as time proceeds and it is continuously converted into material internal energy. There is a slight difference between the velocity of the nodes in the front, middle and the rear of the vehicle, which shows that there is no deformation in the occupant area. As can be seen in Figures 5.21 and 5.22, the nodal velocity for the center and the rear is nearly constant after $65 \mathrm{msec}$; however, the velocity for the front shows some oscillation up to the final solution time. 


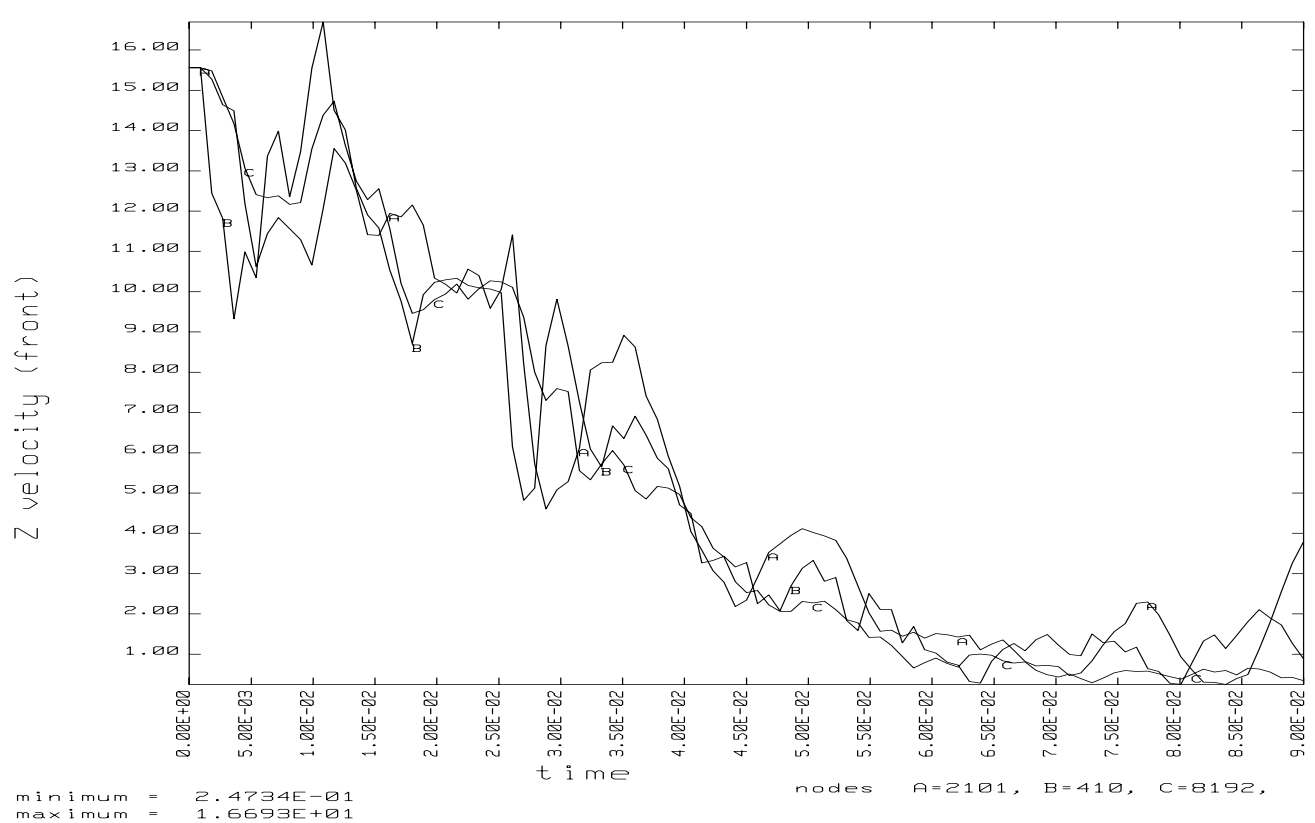

Fig 5.20 $\mathrm{Z}$ velocity for nodes in the front of vehicle.

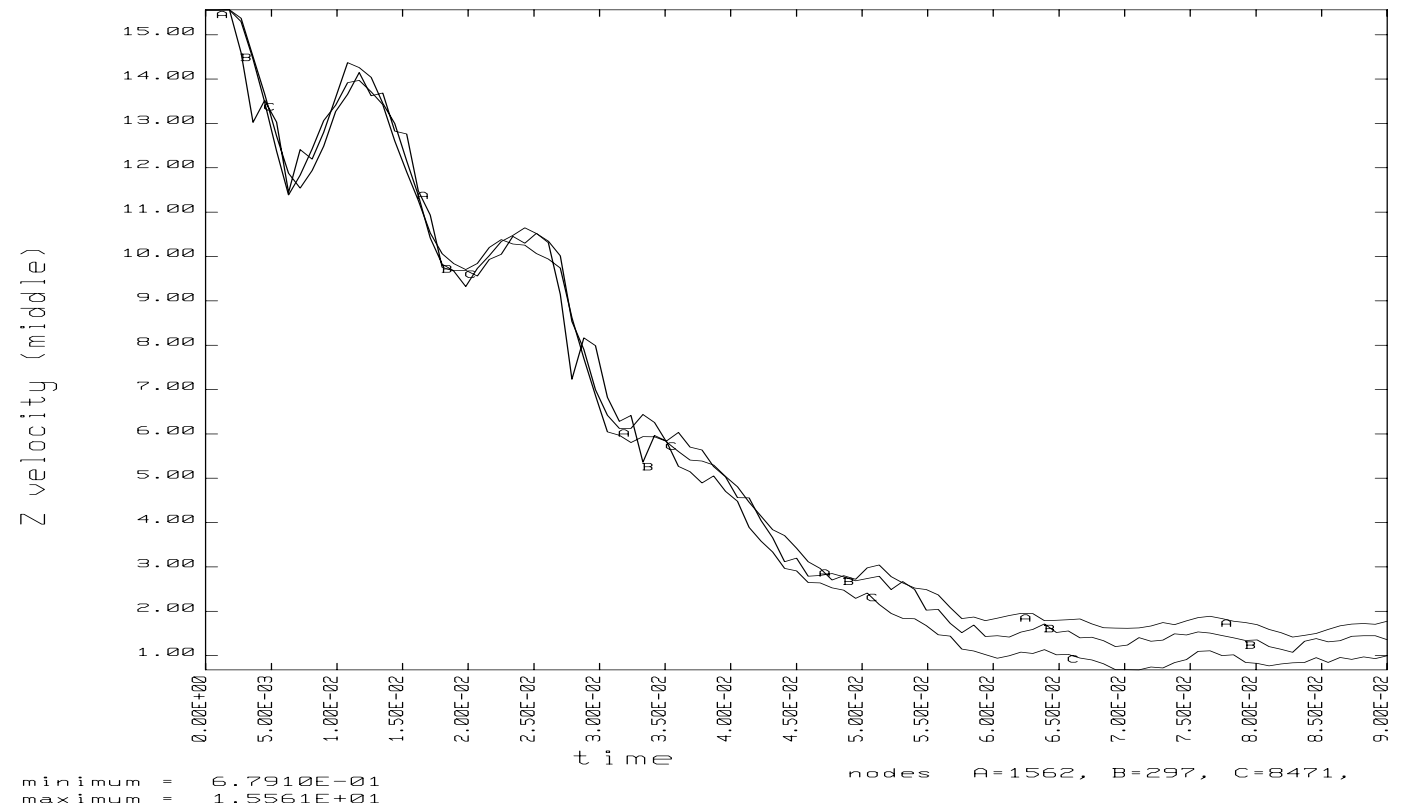

Fig 5.21 $\mathrm{Z}$ velocity for nodes in the middle of vehicle. 


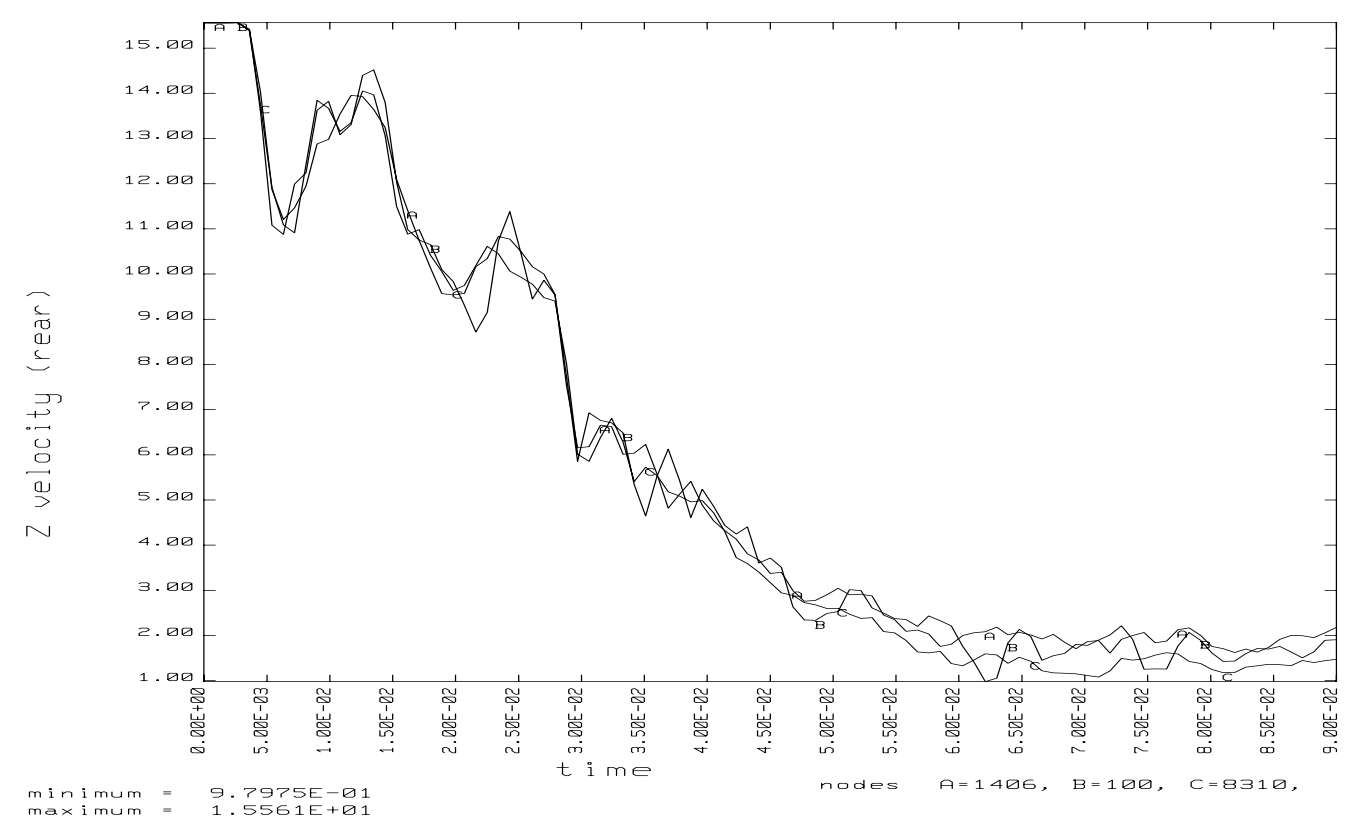

Fig 5.22 $\mathrm{Z}$ velocity for nodes in the rear of vehicle.

Figures 5.23, 5.24, and 5.25 show the $\mathrm{Z}$ acceleration of the nodes in the front, middle, and the rear of the vehicle. The maximum deceleration of the representative node in the front of the vehicle is twice as much as those in the middle and the rear of the vehicle. For the representative nodes, the deceleration in the rear of the vehicle is higher than those in the middle of the vehicle. This shows the bulging of the body skin on the rear side of the vehicle compared to the middle, which is supported by the deformation picture in Figure 5.12. 


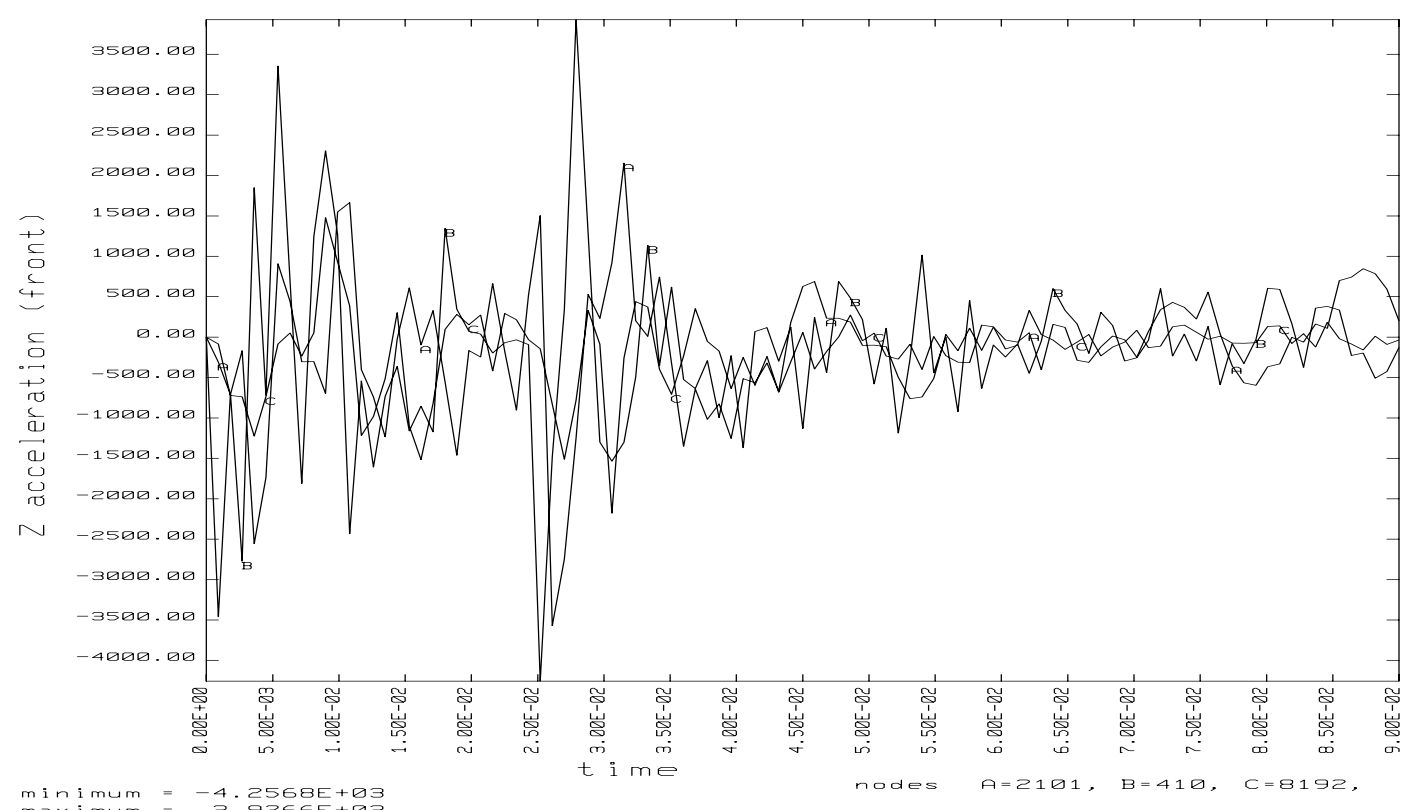

Fig 5.23 $\mathrm{Z}$ acceleration for nodes in the front of vehicle.

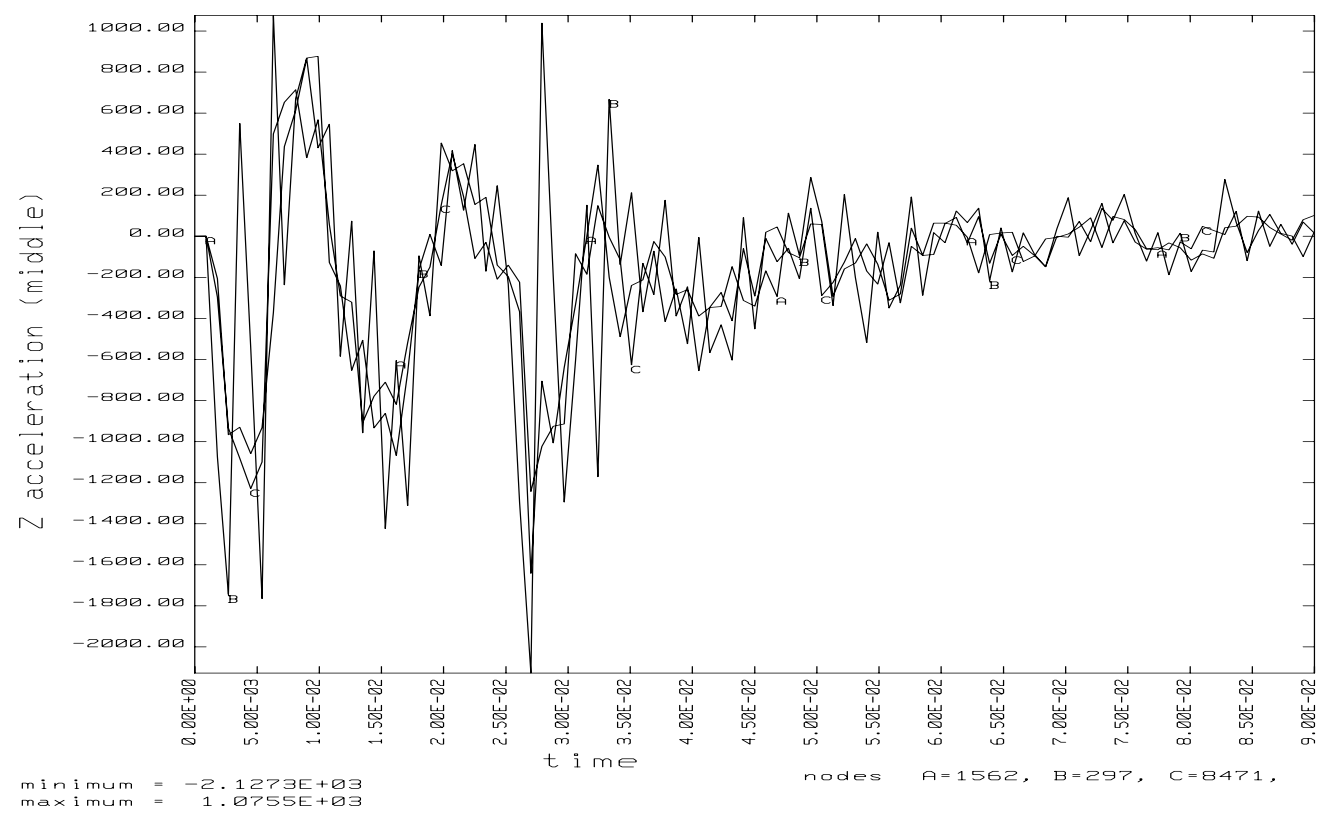

Fig 5.24 $\mathrm{Z}$ acceleration for nodes in the middle of vehicle. 


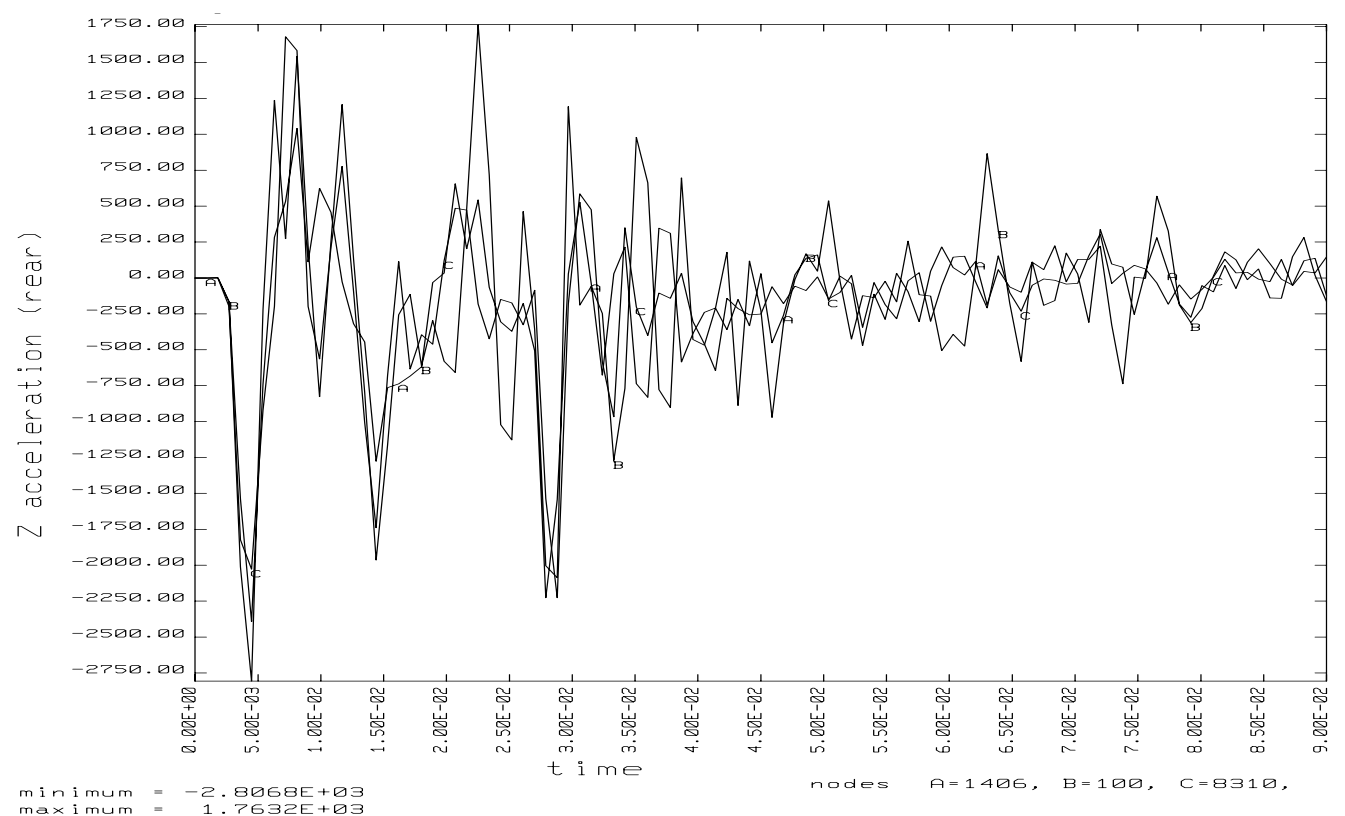

Fig 5.25 $\mathrm{Z}$ acceleration for nodes in the rear of vehicle.

Figure 5.26 shows the $\mathrm{Z}$ rigid body velocity for different parts of the bus that absorb the kinetic energy. The $\mathrm{Z}$ rigid body velocity of the hood reduces at time $=7 \mathrm{msec}$, which shows that the hood has started deforming and there is much less rigid body displacement thereafter. The $\mathrm{Z}$ rigid body velocity of the gear box reduces at time $=25 \mathrm{msec}$, which shows that the gear box has hit the rigid wall and has started absorbing energy. The $\mathrm{Z}$ rigid body velocity of the frame reduces gradually which shows that only a part of the frame undergoes a deformation and the other areas of the frame remain undeformed. The $\mathrm{Z}$ rigid body velocity of the rib structure of the body reduces gradually from $15.55 \mathrm{~m} / \mathrm{sec}$ to $3 \mathrm{~m} / \mathrm{sec}$ at the end of the analysis. The total $\mathrm{Z}$ rigid body velocity of the bus which includes all the components in shown in Figure 5.27. This graph shows that the total rigid body velocity reduces to a constant $1.59 \mathrm{~m} / \mathrm{sec}$ at the end of the analysis. The rigid body velocity in the $\mathrm{X}$ and $\mathrm{Y}$ directions is approximately zero for all the parts. 


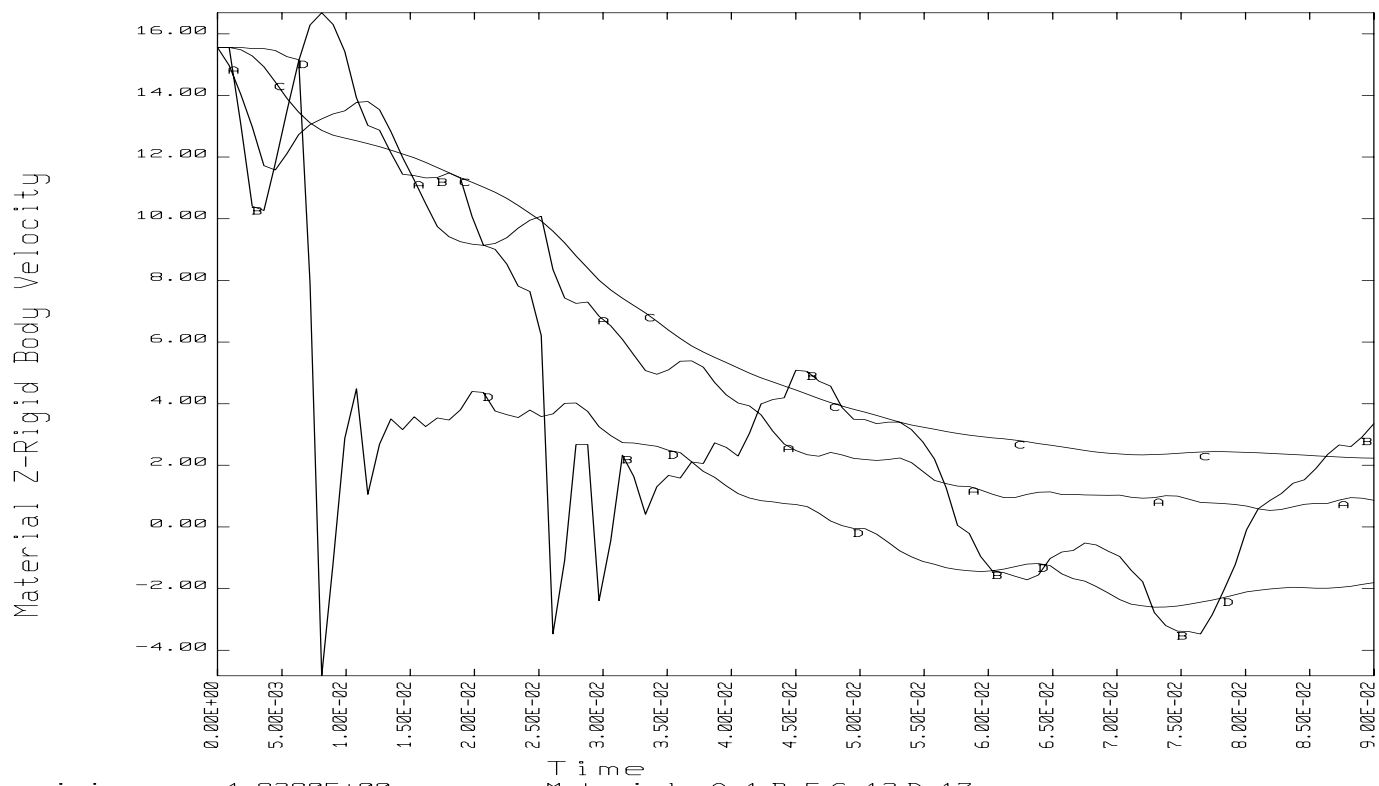

minimum $=-4.828 \nabla E+\square \square$
maximum $=1.6681 E+\square 1$

Fig 5.26 $\mathrm{Z}$ rigid body velocity for materials(Refer to Table 5.1 for table of materials)

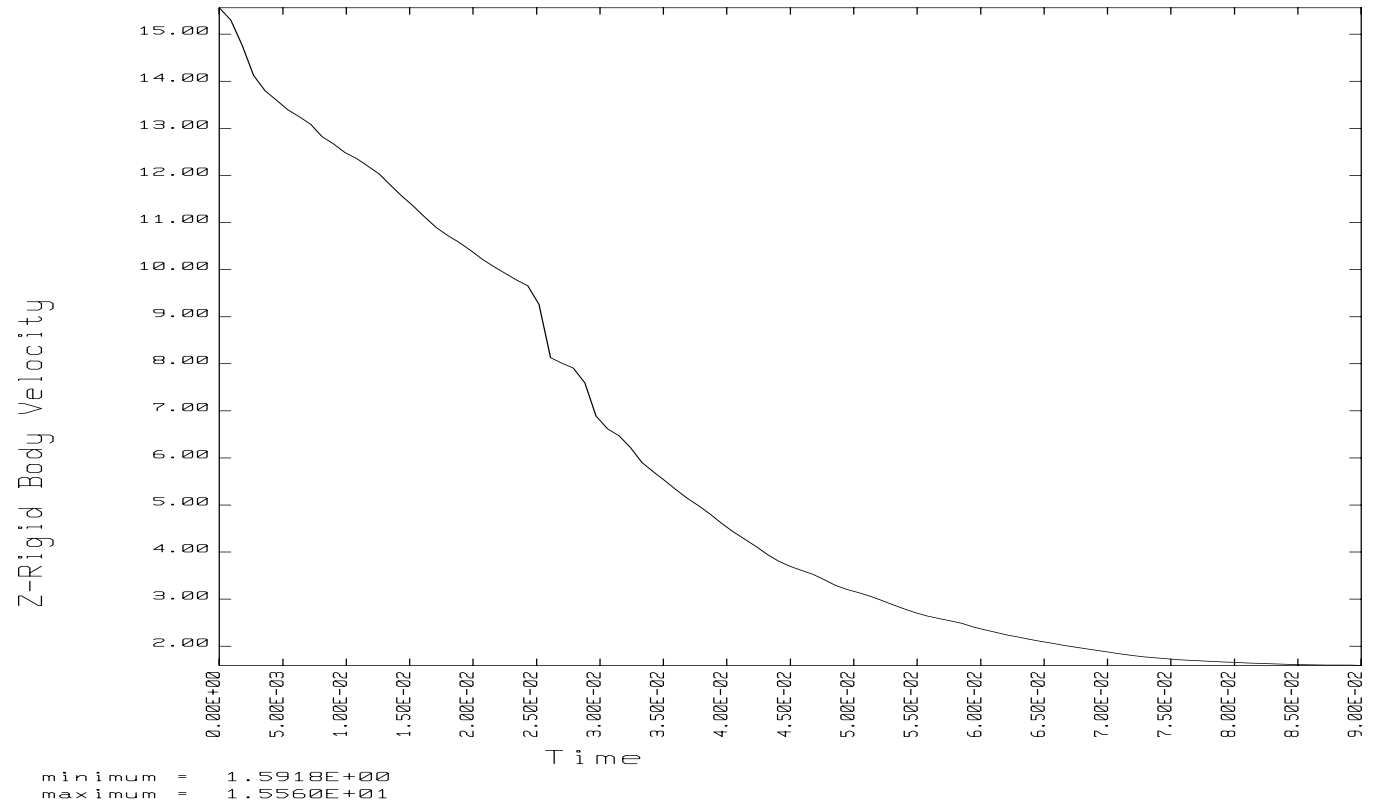

Fig $5.27 \mathrm{Z}$ rigid body velocity for a bus subjected to a full frontal impact at $56 \mathrm{~km} / \mathrm{hr}$.

Figure 5.28 shows the internal energy absorbed by various components of the bus.

The frame (Material 1) absorbs the maximum amount of energy compared to other components like the hood (Material 17), radiator (Material 7), and the body rib structure 
(Material 12). The radiator absorbs more energy next to the frame. Figure 5.30 shows the pie chart of the energy absorbed by different components in the bus for a full frontal impact at the end of the solution. The energy absorbed by the frame is 85 percent of the total internal energy absorbed by all the components of the bus. If the hood, frame, transmission, and the axles were not modeled, then the energy would have been distributed by the other front end components of the bus like the firewall, radiator, and engine. The modeling of the hood and the frame is very important for the frontal impact.

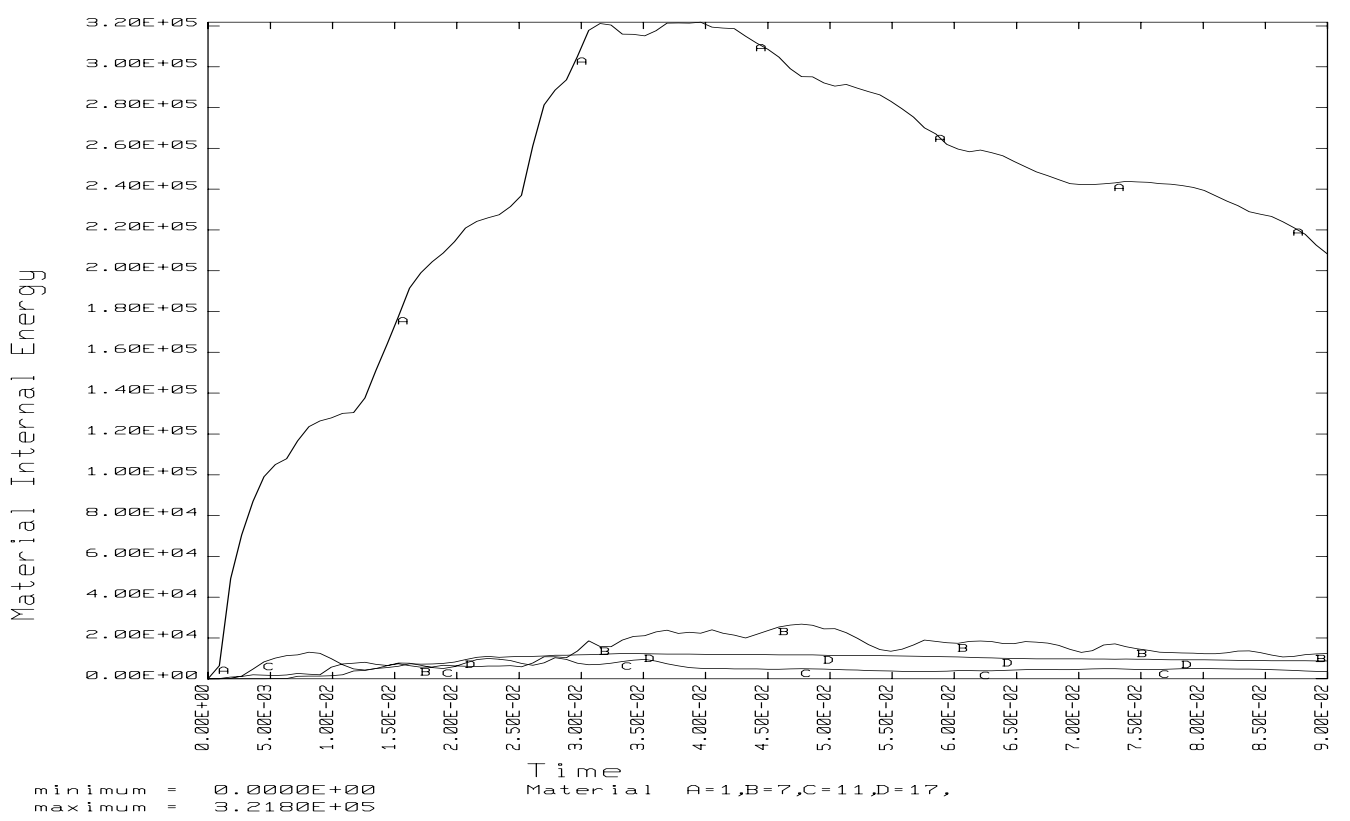

Fig 5.28 Internal energy for materials (refer to Table 5.1 for table of materials).

Figure 5.29 shows the global internal, kinetic, and the total energies of the system. It can be seen that the total energy remains as a constant, which shows that the energy is conserved in the system. At the start of the solution, the internal energy is zero and the kinetic energy is equal to the total energy. When the bus hits the rigid wall, the kinetic energy of the system decreases and it is converted into internal energy of the materials. The kinetic and the internal energies meet at $30 \mathrm{msec}$, and the kinetic energy continues to 
decrease while the internal energy continues to increase. To reduce the hourglass energy in the system, eight point integration was done for the brick elements. To reduce the hour glass energy for thin shell elements, a fully integrated Hughes Liu thin shell element has to be used, which is CPU costly and increases the solution time by six times. So, the hour glass energy present in the model is only due to the thin shell elements. The internal and the kinetic energy curves should meet at a point exactly equal to half the total energy and continue to stay constant at that point without crossing, for the rest of the analysis. In the case of the full frontal impact, the curves meet at time $=30 \mathrm{msec}$, and they cross each other and continue to remain constant. The remaining energy, other than the sum of the internal and the kinetic energies, is the hourglassing energy due to the thin shell elements.

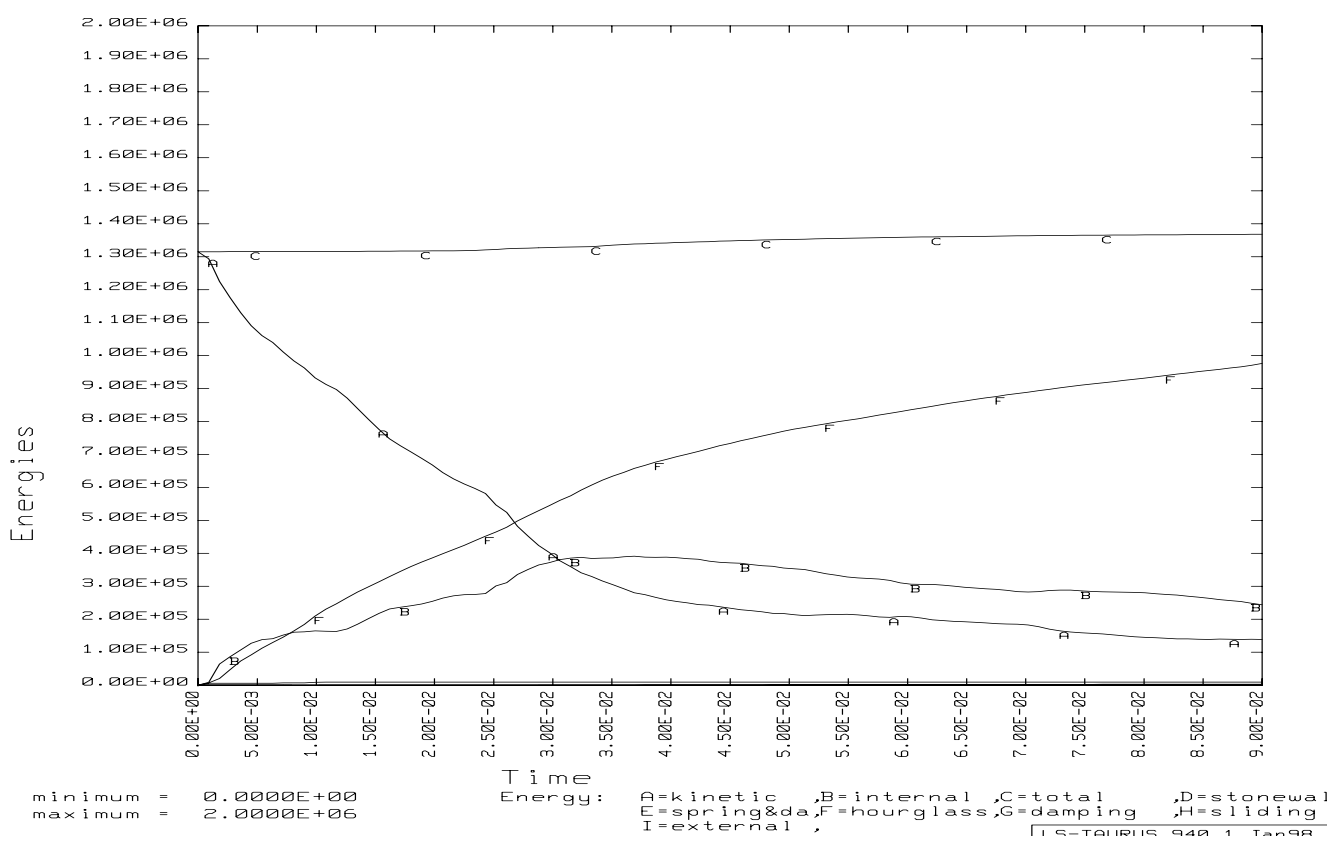

Fig 5.29 Internal, kinetic, and the total energies for a full frontal impact. 


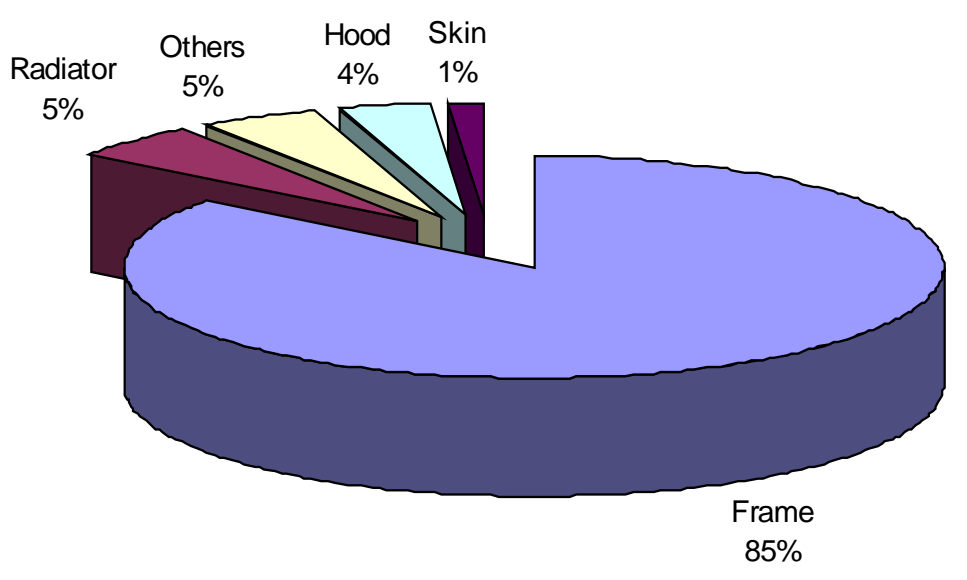

Fig 5.30 Total internal energy distribution at time $=90 \mathrm{msec}$ of the bus subjected to a full frontal impact.

\subsection{OfFSET FRONTAL IMPACT WITH BELYTSCHKO -TSAY THIN SHELL ELEMENTS}

The offset frontal impact was simulated for the bus with an initial velocity of 56 $\mathrm{km} / \mathrm{hr}$. The rigid wall was defined for only the right side (door side) of the hood and the rigid wall extends to infinity in that direction. The left side (driver side) of the bus does not have a rigid wall and is free to undergo any deformation. This scenario represents the impact of the school bus against a corner of a building or a concrete barrier.

The front view of the bus at time $=9$ msec subjected to an offset frontal impact is shown in Figure 5.31. The bumper has hit the rigid wall defined on only one side of the vehicle (door way) as illustrated in the picture. The kinetic energy of the vehicle reduces and it is absorbed as internal energy by the bumper. To reduce the hourglassing energy for the solid elements, an eight point integration was done and the hourglassing energy in the system is only due to the thin shell elements. 


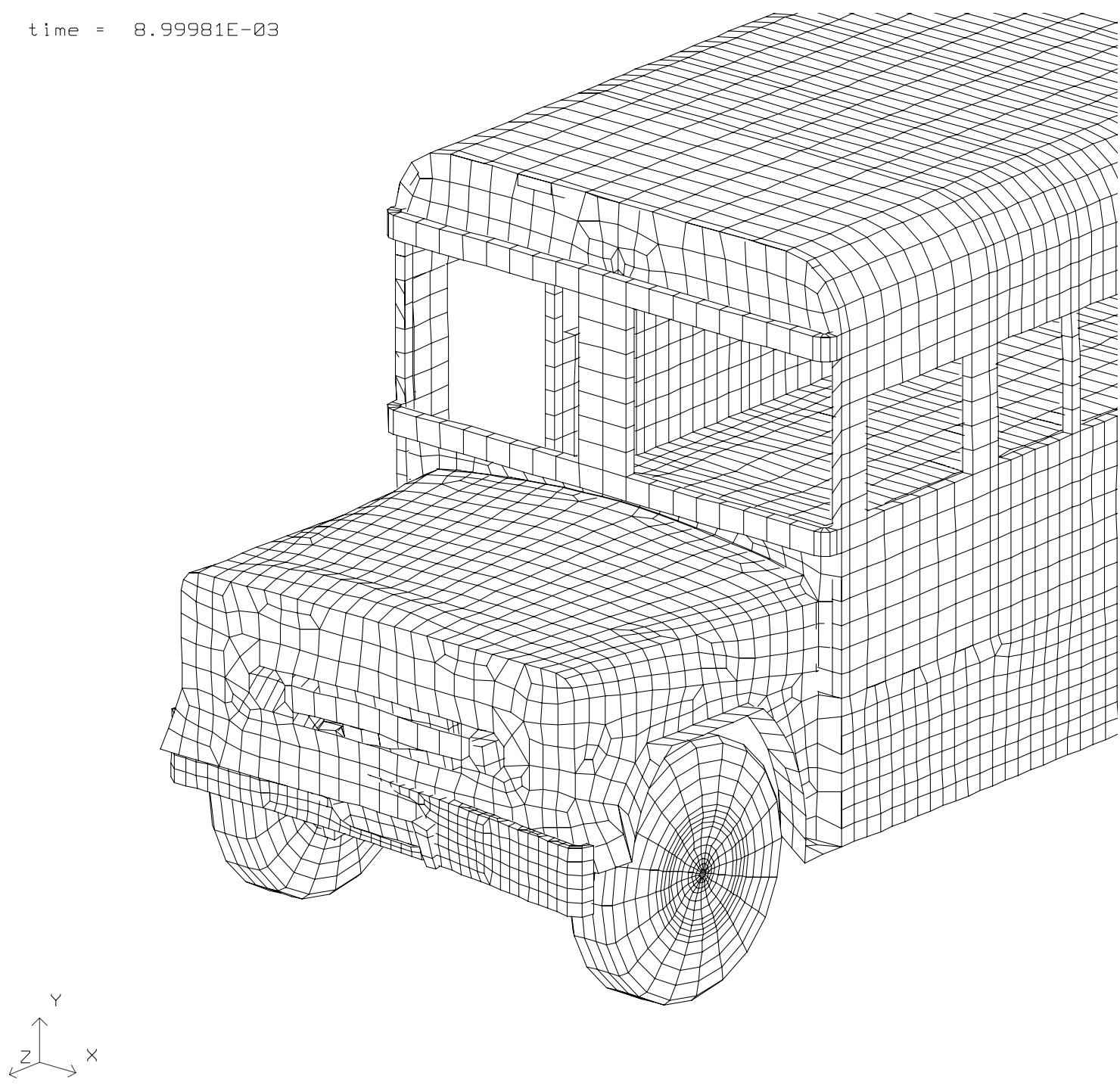

Fig 5.31 Front view at time $=9 \mathrm{msec}$ of the bus subjected to an offset frontal impact at a velocity of $56 \mathrm{~km} / \mathrm{hr}$.

The deformation of the hood on only one side can be seen in Figure 5.32. The left side of the bus does not undergo any deformation since the rigid wall is only on one side and there is a rigid body displacement of the front axle on the left side. The rotation of the front axle can be seen from the graph showing the $\mathrm{Z}$ relative displacement between the nodes in the right and the left tire in Figure 5.41. 


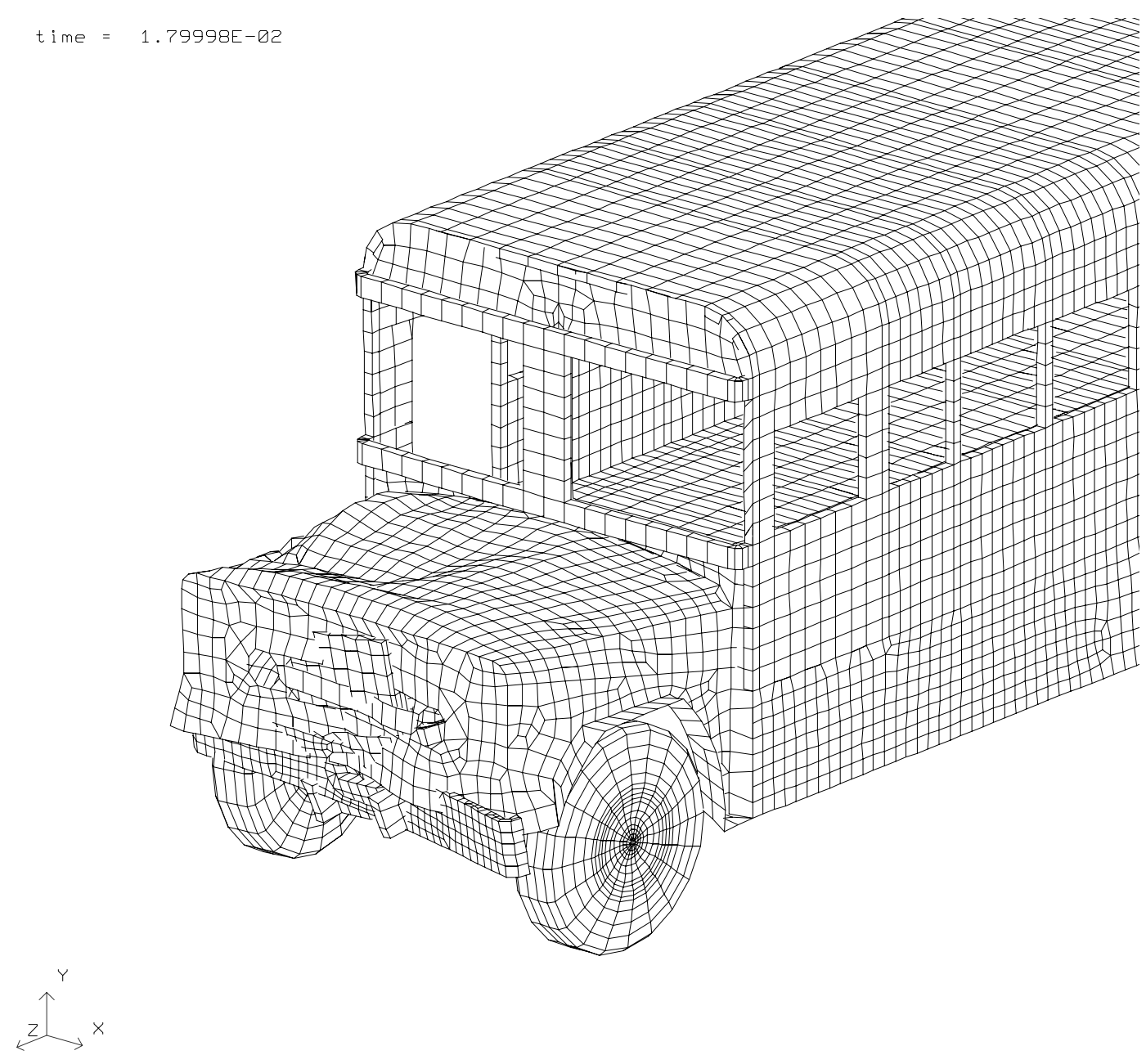

Fig 5.32 Front view at time $=18 \mathrm{msec}$ of the bus subjected to an offset frontal impact at a velocity of $56 \mathrm{~km} / \mathrm{hr}$.

Figure 5.33 shows the front view at time $=27 \mathrm{msec}$ of the bus subjected to an offset frontal impact against a rigid wall. The difference in $\mathrm{Z}$ displacement between the nodes in the right tire (door side) and the left tire (driver side) increases, which shows the rotation of the front axle due to an offset in the principal direction of force and as seen in Figure 5.41. The sum of the kinetic and the internal energies is not equal to the total energy because of the presence of hourglassing energy in the system. This can be seen from the graph in Figure 5.55. 


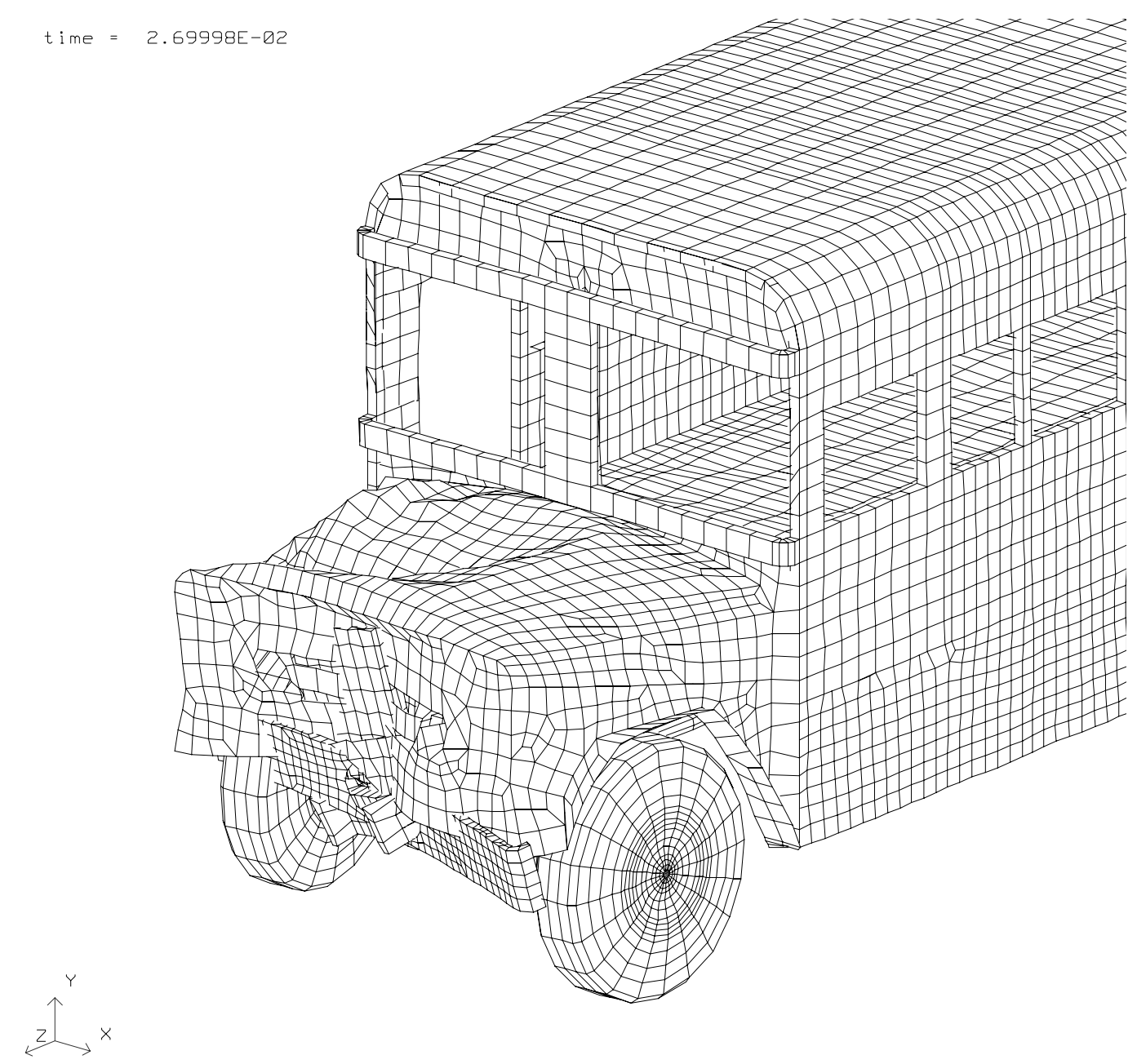

Fig 5.33 Front view at time $=27 \mathrm{msec}$ of the bus subjected to an offset frontal impact at a velocity of $56 \mathrm{~km} / \mathrm{hr}$.

The front view of the bus subjected to an offset frontal impact at time $=36 \mathrm{msec}$ is shown in Figure 5.34. The difference in $\mathrm{Z}$ displacement between the nodes in the right tire (door side) and the left tire (driver side) continuously increases, which shows that the door side of the front end only experiences the impact. This can be seen from the graph in the Figure 5.41. The front tire moves down in the $\mathrm{Y}$ axis compared to the rear tire and the vehicle pitches about the $\mathrm{X}$ axis, which can be seen from the graph showing the relative displacement between the front and the rear tires in Figure 5.42. The maximum stress in the firewall is $164 \mathrm{MN} / \mathrm{m}^{2}$, which is within the yield stress of the material of the firewall; 
and the absolute displacement of the fire wall is $0.48 \mathrm{~m}$ in the positive $\mathrm{Z}$ direction, which shows that there is no intrusion in the occupant area in the door side.

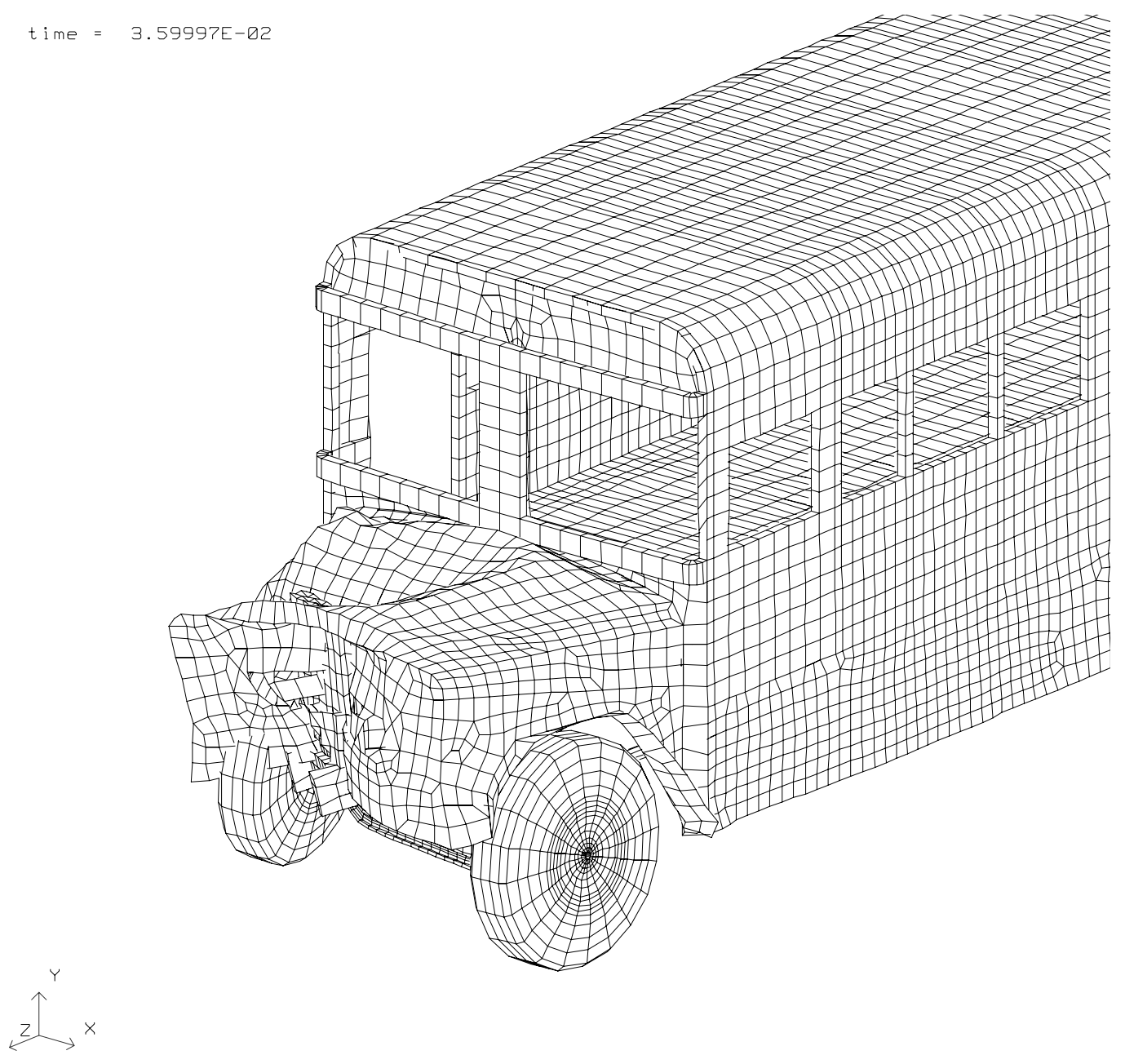

Fig 5.34 Front view at time $=36 \mathrm{msec}$ of the bus subjected to an offset frontal impact at a velocity of $56 \mathrm{~km} / \mathrm{hr}$.

Figure 5.35 shows the picture of the bus at time $=45 \mathrm{msec}$ subjected to an offset frontal impact. The frame absorbs the maximum internal energy, which can be seen in Figure 5.54. The acceleration of the nodes in the front, middle, and the rear of the vehicle oscillates about $0 \mathrm{~m} / \mathrm{sec}^{2}$ after time $=45 \mathrm{msec}$, which shows that the solution is approaching a steady state. This can be seen from Figures 5.50, 5.51, and 5.52. 


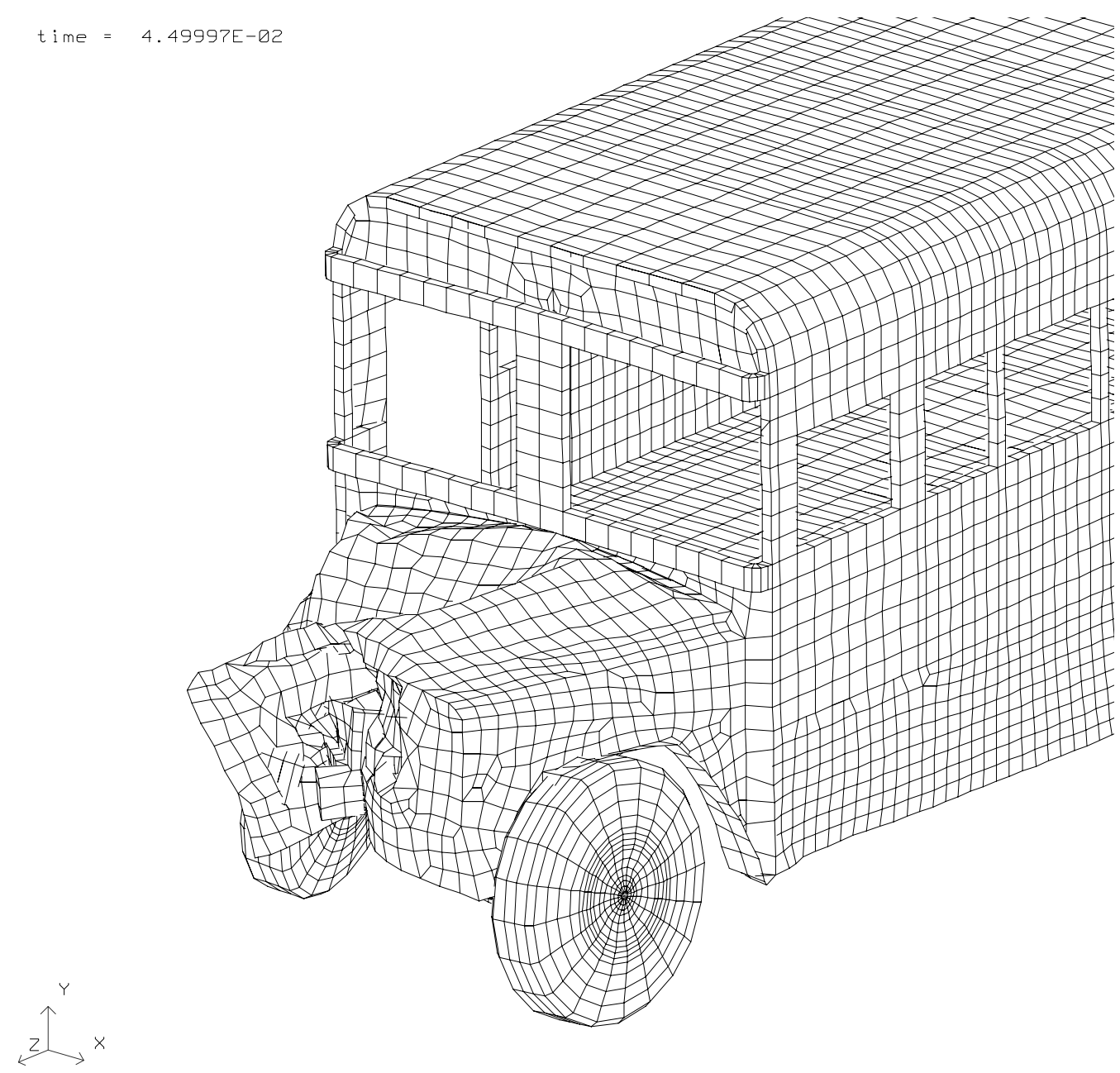

Fig 5.35 Front view at time $=\mathbf{4 5} \mathrm{msec}$ of the bus subjected to an offset frontal impact at a velocity of $56 \mathrm{~km} / \mathrm{hr}$.

Figure 5.36 shows the picture of the bus subjected to a offset frontal impact at time $=54$ msec. The deformation increases only on the door side of the body and the hood rotates about the $\mathrm{Y}$ axis with the driver side end undergoing a rigid body displacement. The kinetic energy of the bus lessens due to the deceleration and the internal energy increases as seen in Figure 5.55. The frame absorbs the maximum amount of internal energy followed by the radiator as seen in Figure 5.54. The maximum stress of 167 $\mathrm{MN} / \mathrm{m}^{2}$ in the fire wall is at the door side of the bus and the maximum absolute displacement of the fire wall is $0.6 \mathrm{~m}$. 


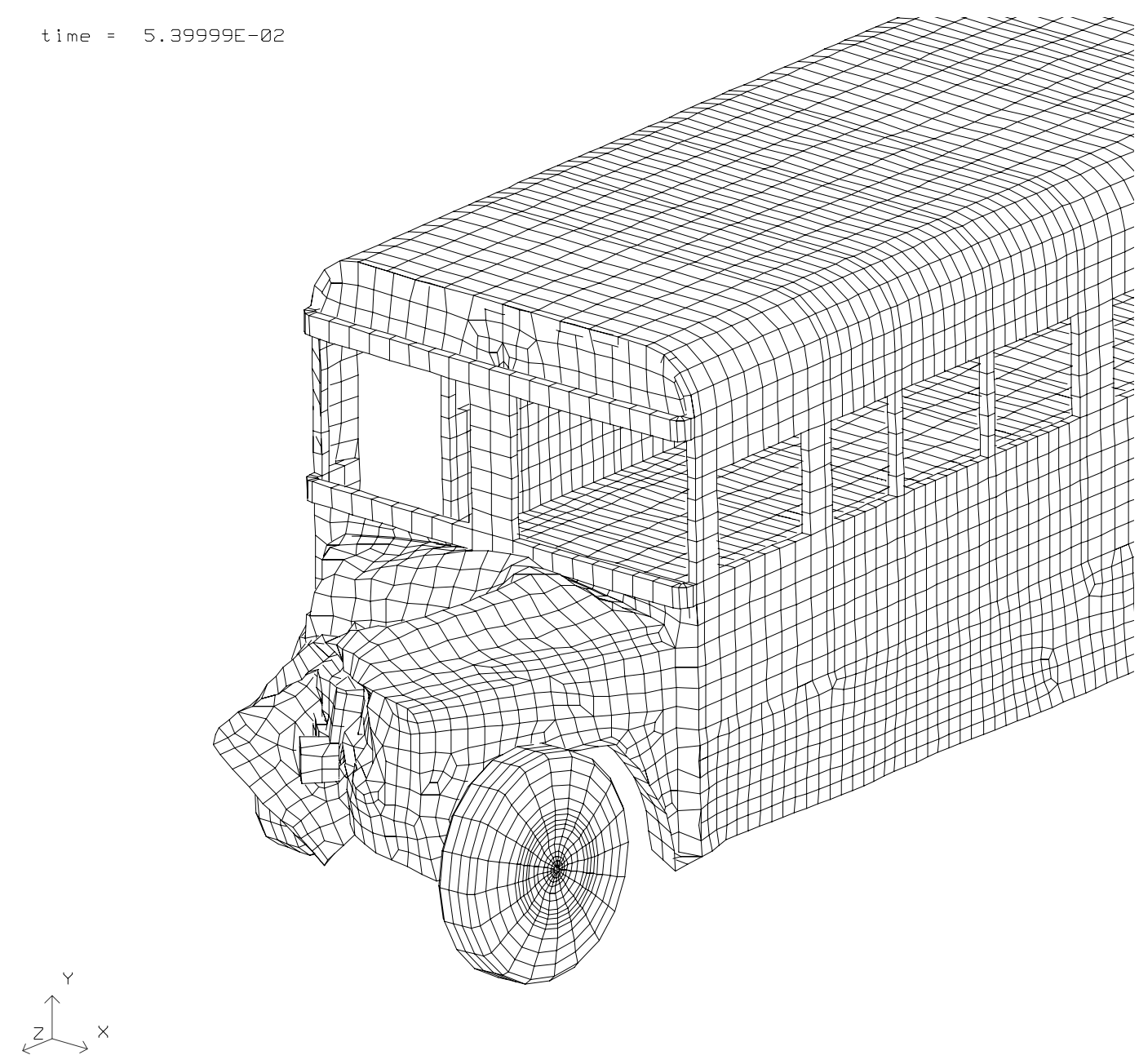

Fig 5.36 Front view at time $=54 \mathrm{msec}$ of the bus subjected to an offset frontal impact at a velocity of $56 \mathrm{~km} / \mathrm{hr}$.

Figure 5.37 shows the front view of the chassis of the bus subjected to an offset frontal impact at time $=72 \mathrm{msec}$. The front end of the frame rises up with the radiator and the engine and bends to the right side where the rigid wall was defined. The propeller shaft also bends and sways in the center because the bolts that attach the propeller shaft to the frame were not modeled. The relative displacement between the node on the right end of the firewall and the right side tire increases, which shows that the right tire hits the fire wall and the body skin near the door way as seen in Figure 5.43. This also shows a protrusion of the tire into the doorway area. 


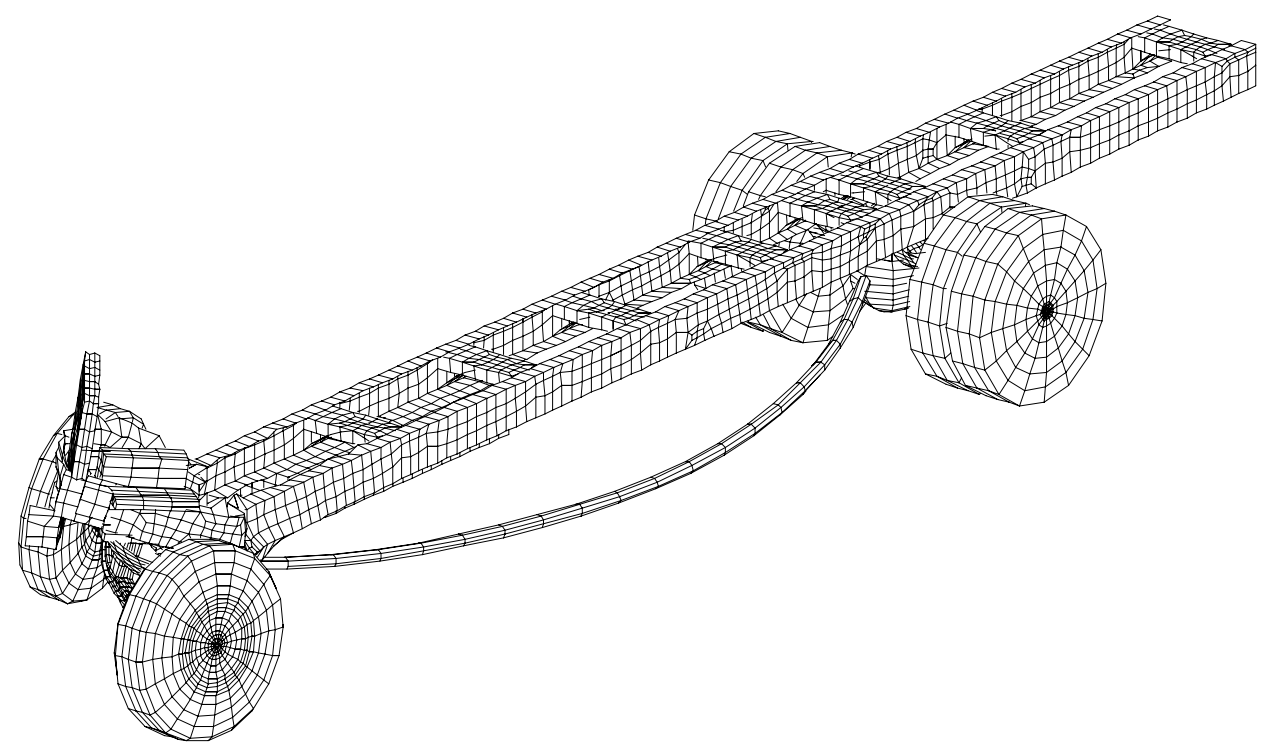

Fig 5.37 Front view at time $=72 \mathrm{msec}$ of the bus subjected to an offset frontal impact at a velocity of $56 \mathrm{~km} / \mathrm{hr}$.

Figure 5.38 shows the front view of the bus subjected to an offset frontal impact at time $=90$ msec. The only intrusion in the occupant area is the deformation of the fire wall next in the area near the doorway. The maximum stress in the hood is $1570 \mathrm{MN} / \mathrm{mm}^{2}$, which is higher than the yield stress of the hood and it shows that the deformation is plastic. The maximum $\mathrm{Z}$ displacement on the fire wall is 0.8 meters at the end of the analysis. The energy absorbed by the frame is maximum for all the time states of the solution. The side view and the top view of the bus at time $=90 \mathrm{msec}$ is shown in Figure 5.39 and Figure 5.40, respectively. Figure 5.39 shows the pitching of the vehicle. 


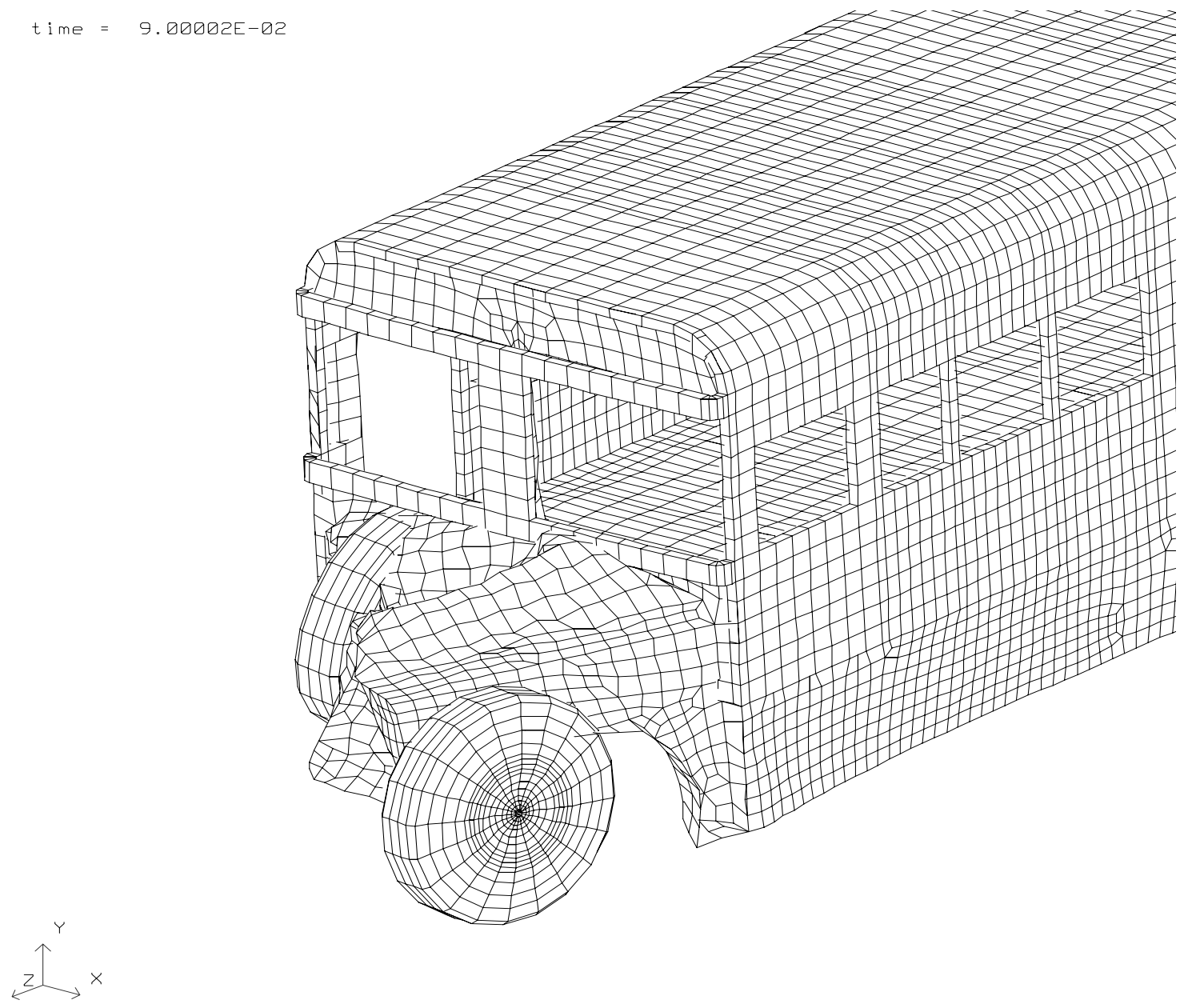

Fig 5.38 Front view at time $=90 \mathrm{msec}$ of the bus subjected to an offset frontal impact at a velocity of $56 \mathrm{~km} / \mathrm{hr}$. 


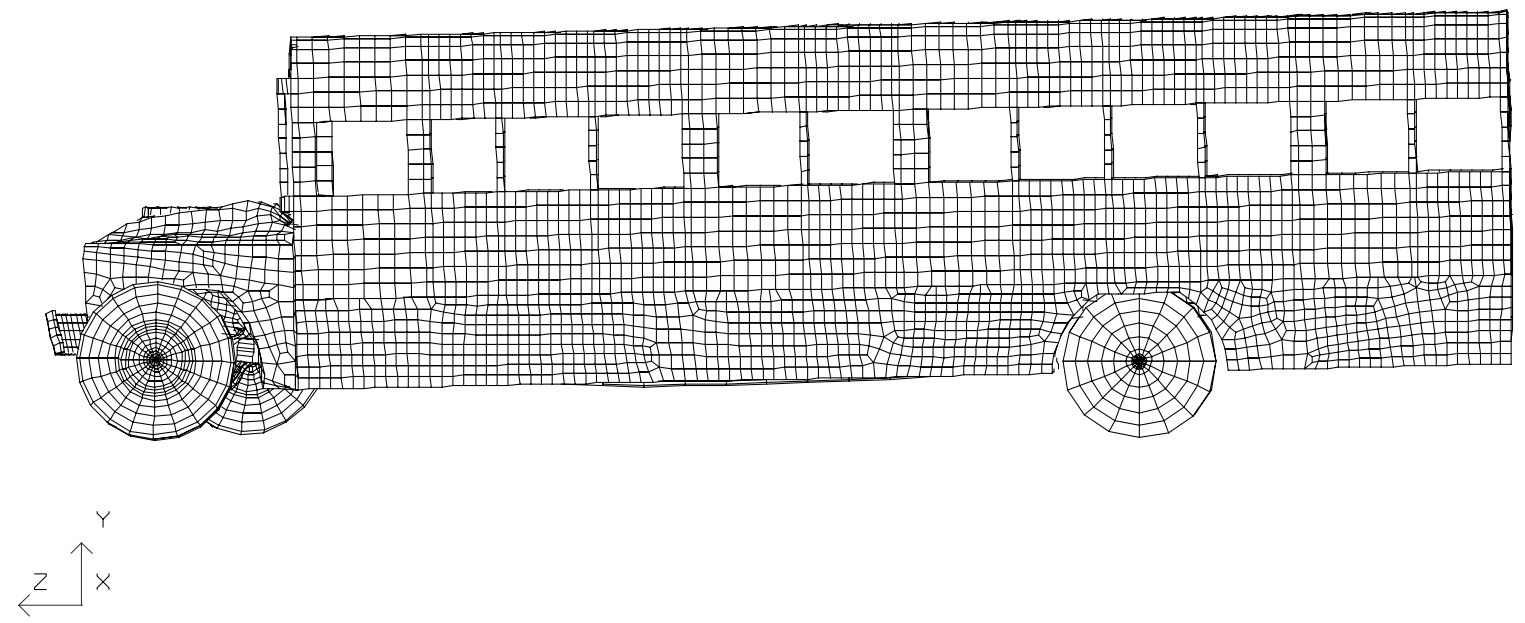

Fig 5.39 Side view at time $=45$ msec of the bus subjected to an offset frontal impact.

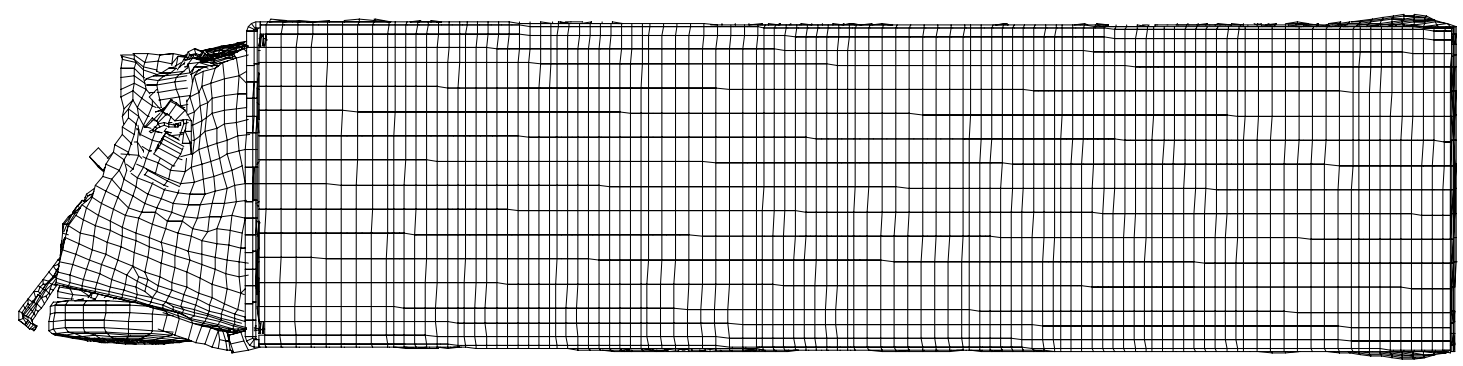

$\stackrel{Z}{\longleftarrow}{ }_{x}^{Y}$

Fig 5.40 Top view at time $=45$ msec of the bus subjected to an offset frontal impact. 
Figure 5.41 shows the $\mathrm{Z}$ relative displacement between the nodes in the left tire (driver side) and the right tire (door side). The relative displacement continuously increases which shows that the right tire due to the offset impact undergoes a rigid body displacement and the front axle rotates about the $\mathrm{Y}$ axis. This can also be seen from Figure 5.40. Figure 5.42 shows the $\mathrm{Y}$ relative displacement between the nodes in the front tire and the rear tire on the left side. The relative displacement remains small till 65 msec and then it increases rapidly. This shows that the vehicle starts to pitch only after 65 msec. Figure 5.43 shows the relative displacement between the nodes in the right side of the fire wall and the right tire. The relative displacement increases in the negative $\mathrm{Z}$ direction, which shows that the right wheel is fast approaching the fire wall. This is due to the axle rotation about the y axis due to an offset impact force at one side of the bus.

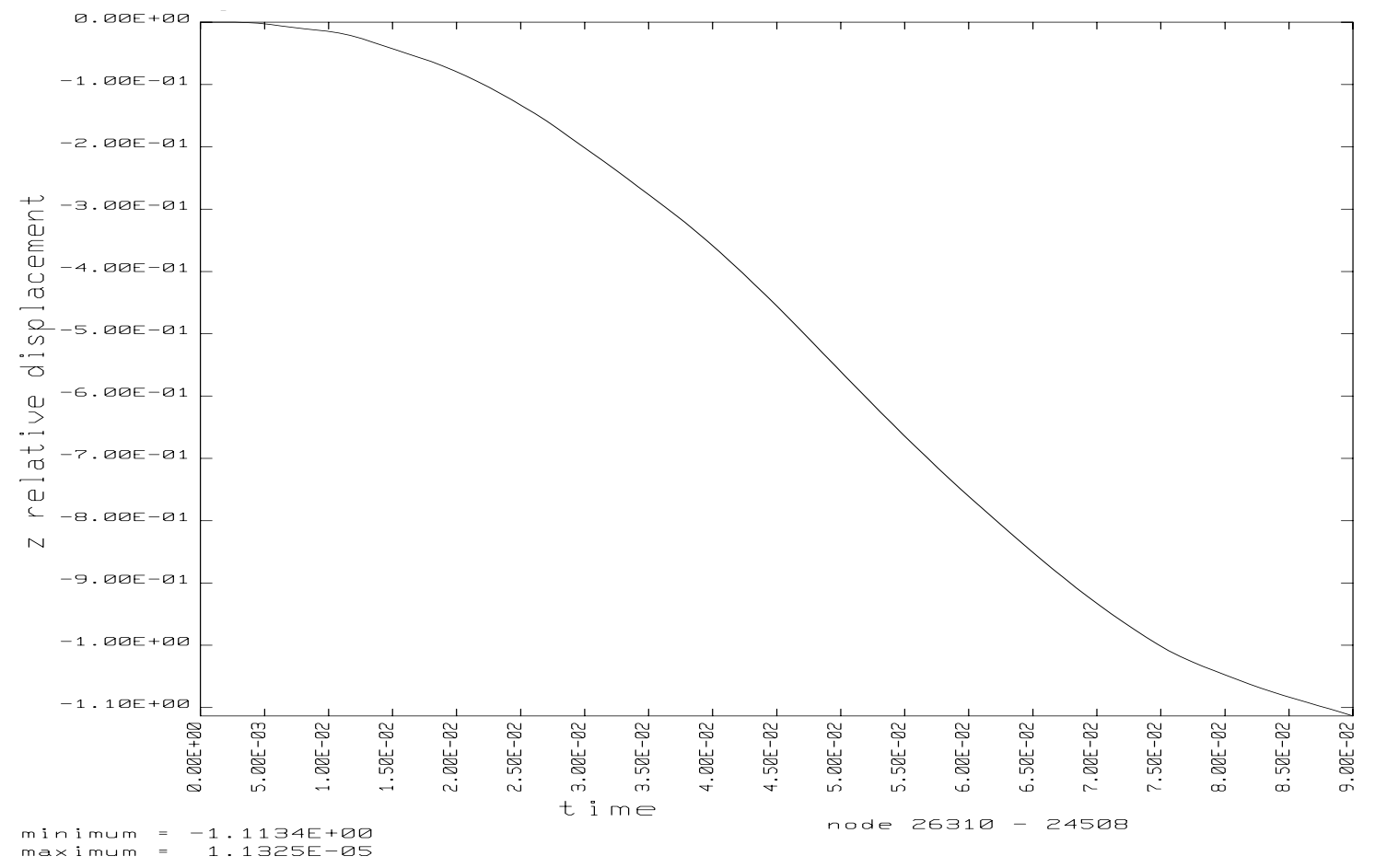

Fig 5.41 Relative displacement between the nodes in the right and the left tires in front axle. 


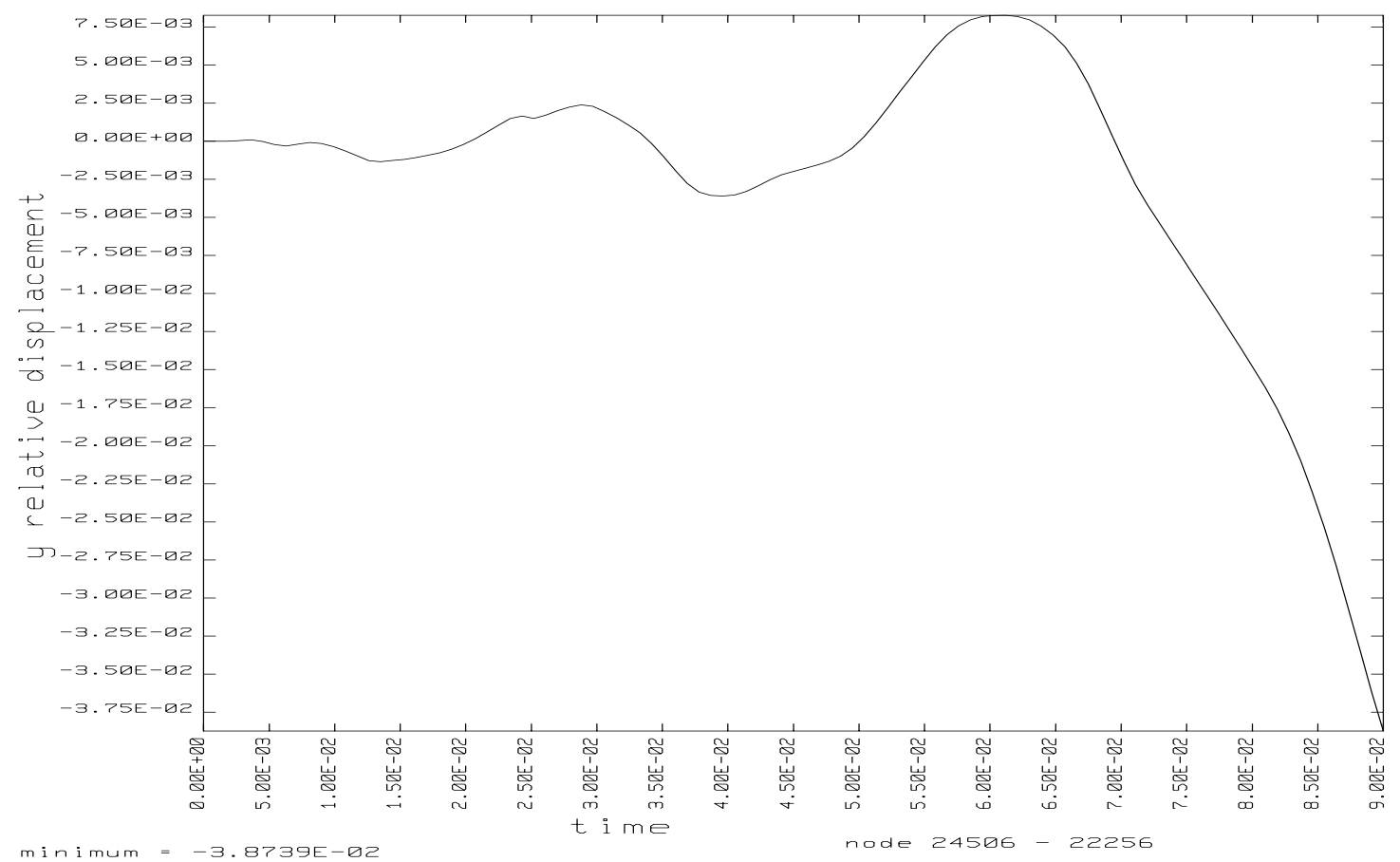

minimum $=-3.8739 E-\square 2$

Fig 5.42 Relative displacement between the front tire and the rear tire.

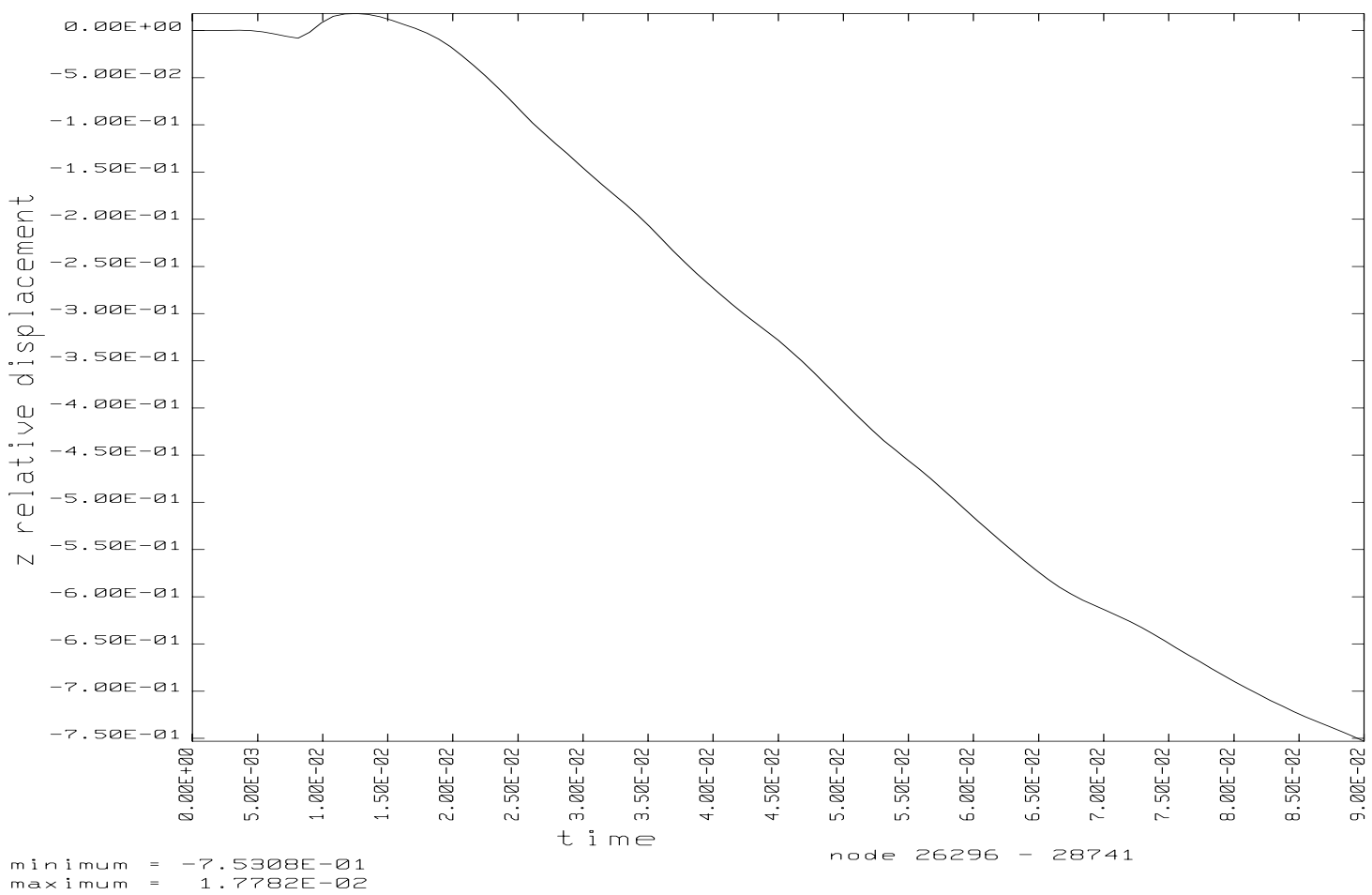

Fig 5.43 Relative displacement between the nodes in the fire wall and the right tire. 
Figures 5.44, 5.45, and 5.46 show the $\mathrm{Z}$ displacement for the selected nodes in the front, middle, and the rear of the vehicle. The front of the vehicle is the position along the $\mathrm{X}$ axis from the driver seat to the doorway in the front, the middle of the vehicle is the center position between the rear end and the driver seat end of the vehicle ( $\mathrm{X}$ axis), and the rear of the vehicle is the position at the rear end of the vehicle ( $\mathrm{X}$ axis) where the indicators are positioned. The nodes were chosen to represent the left skin, center floor, and the left skin for all the three portions in the bus. The graphs show that the maximum $\mathrm{Z}$ displacement of the nodes in the middle of the vehicle and the rear of the vehicle is greater than the maximum $\mathrm{Z}$ displacement of the nodes in the front of the vehicle. This is due to the front end of the vehicle experiencing the impact, and the deformation in the $\mathrm{Z}$ axis for the body structure is the difference between the maximum displacements in the front and the middle of the vehicle.

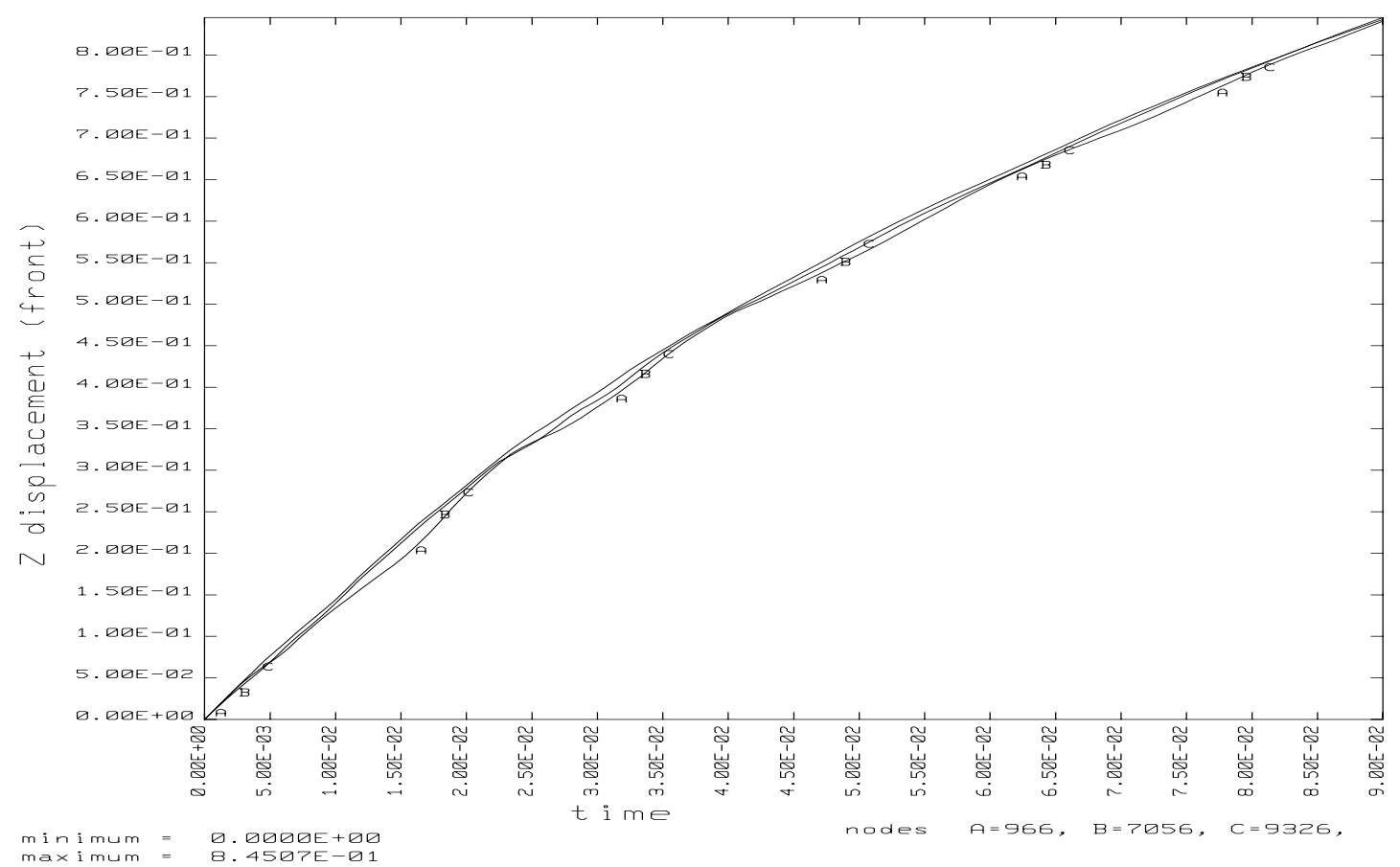

Fig 5.44 $\mathrm{Z}$ displacement for the nodes in the front of the vehicle. 


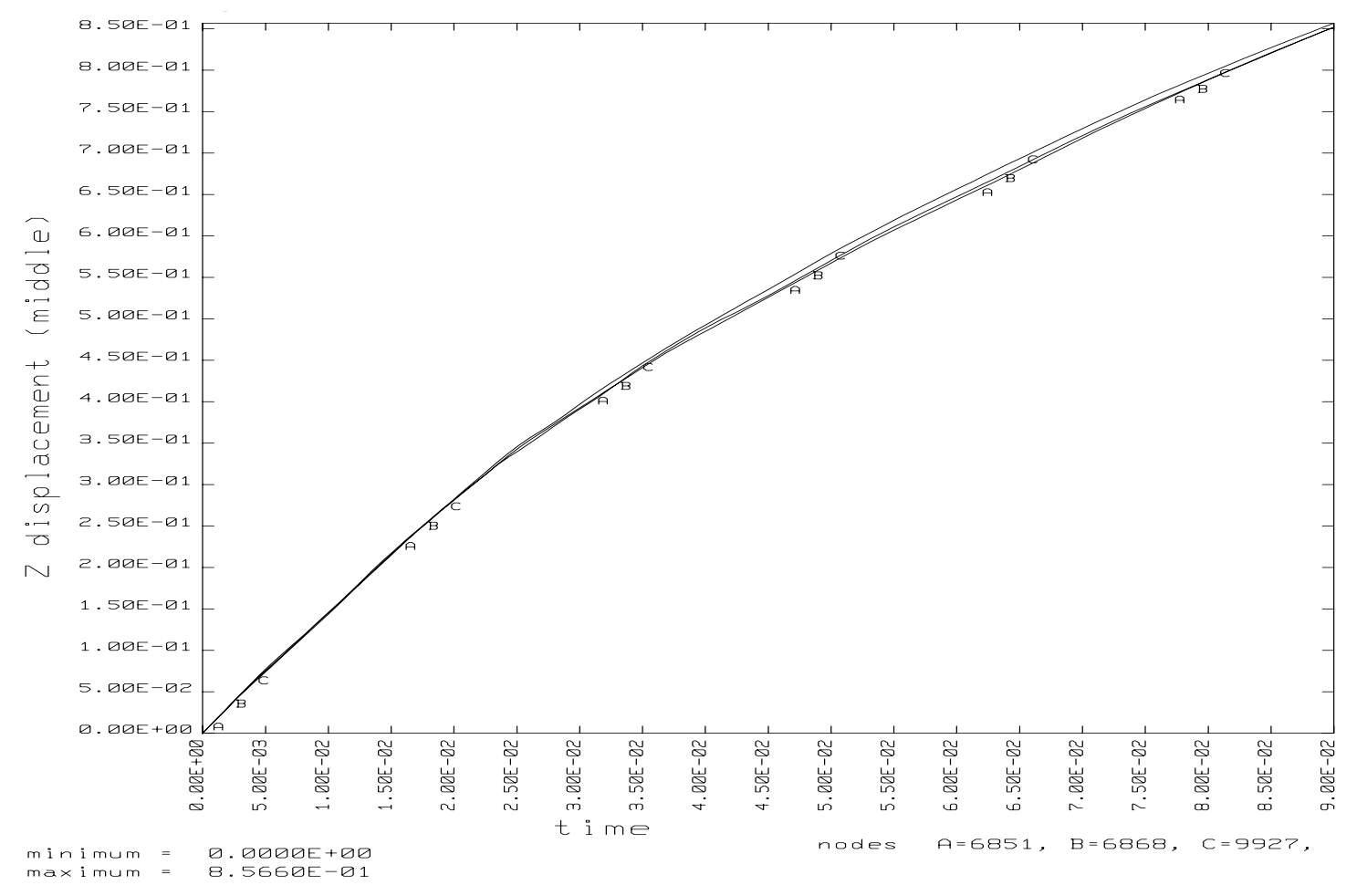

Fig 5.45 $\mathrm{Z}$ displacement for the nodes in the middle of the vehicle.

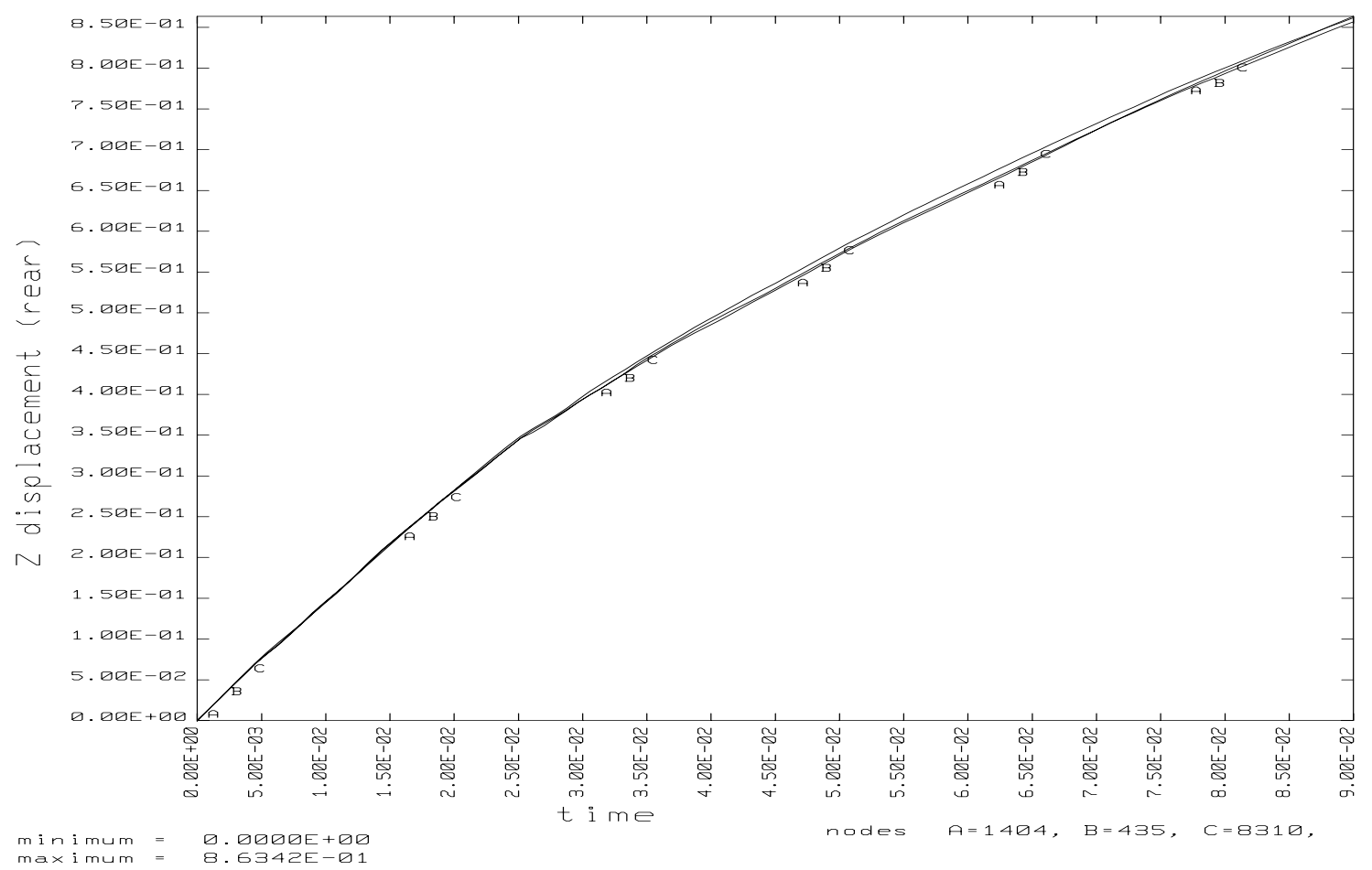

Fig 5.46 $\mathrm{Z}$ displacement for the nodes in the rear of the vehicle. 
Figure 5.47, 5.48, and 5.49 show the $\mathrm{Z}$ velocity for the nodes in the front, middle, and rear of the vehicle. From Figure 5.47, which shows the velocity of the nodes in the front of the vehicle, it can be seen that the velocity for node 966 decreases at a higher rate compared to the other nodes because it lies on the right side of the front end that undergoes the offset impact. The velocity of the nodes in the middle and the rear of the vehicle is the same, which shows that the deformation in the rear end of the vehicle is much less. The velocity of these points is to $6 \mathrm{~m} / \mathrm{sec}$ at $90 \mathrm{msec}$; it appears that a greater solution time is needed to reach a constant velocity from these three figures.

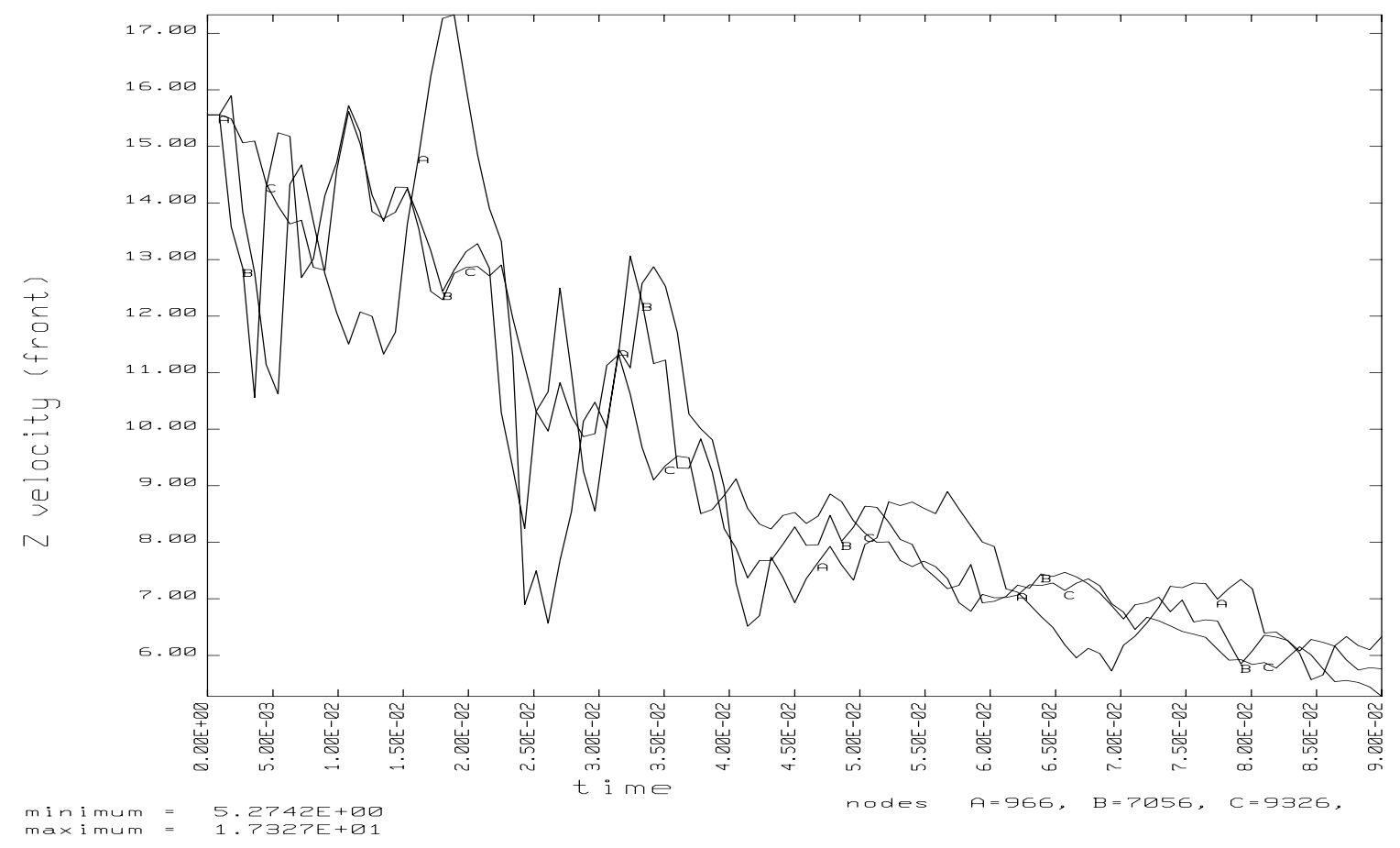

Fig 5.47 $\mathrm{Z}$ velocity for the nodes in the front of the vehicle. 


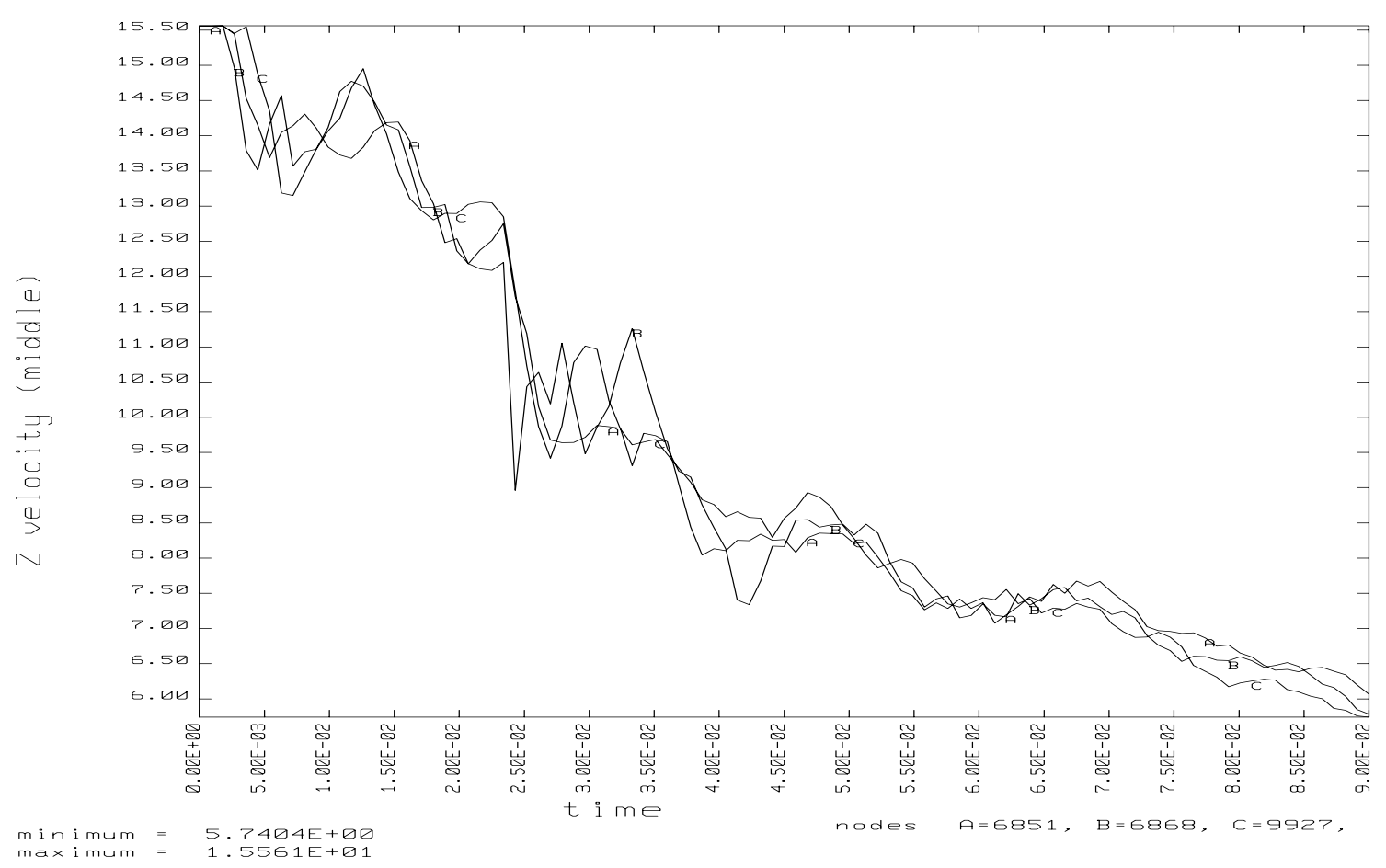

Fig 5.48 $\mathrm{Z}$ velocity for the nodes in the middle of the vehicle.

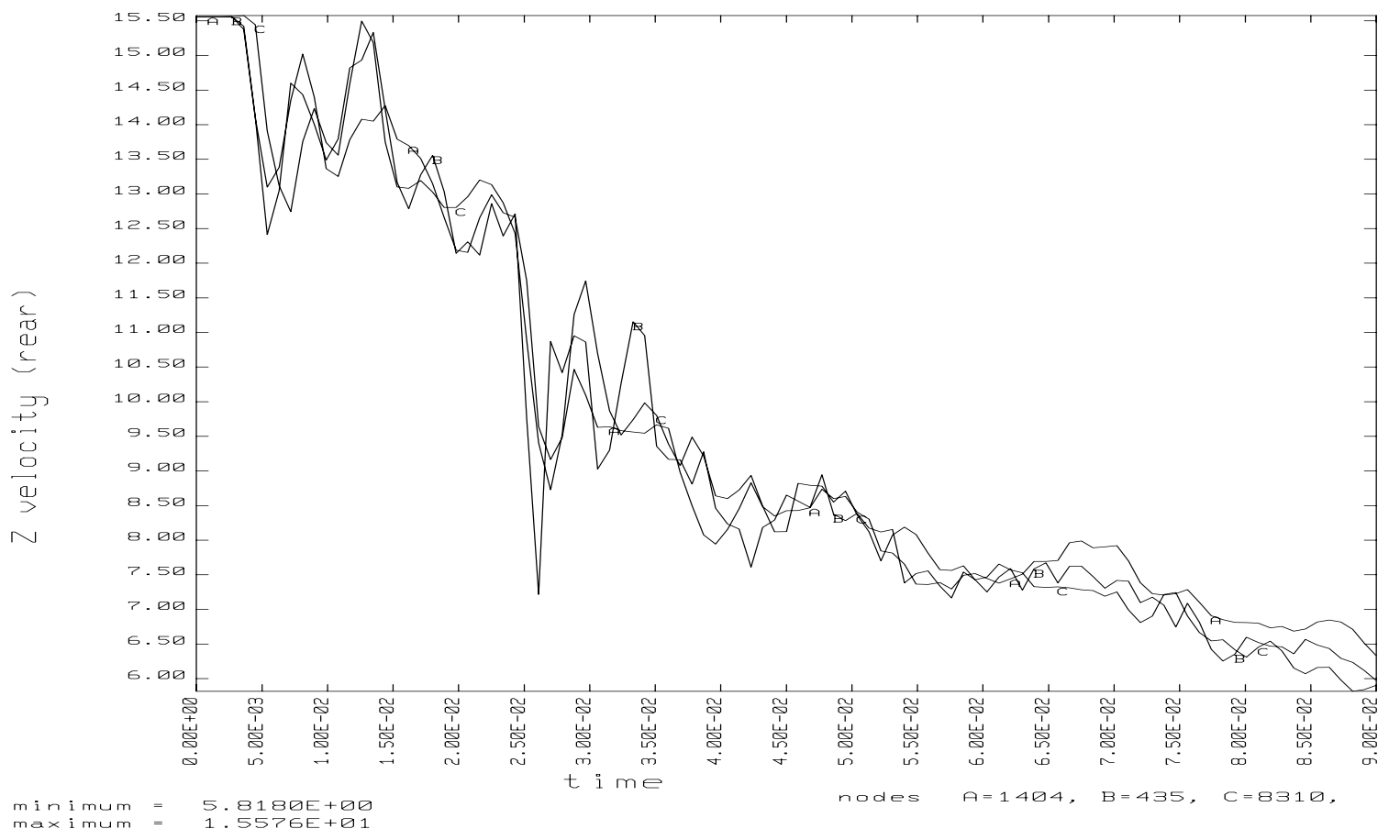

Fig 5.49 $\mathrm{Z}$ velocity for the nodes in the rear of the vehicle. 
Figure 5.50, 5.51, and 5.52 show the $\mathrm{Z}$ acceleration for the nodes in the front, middle, and rear of the vehicle. From Figure 5.50, it can be seen that the maximum deceleration for node 966 is higher than the other nodes in the front of the vehicle. This is because node 966 lies on the right end of the front of the vehicle near the doorway that experiences the offset impact. From Figure 5.51, it can be seen that the maximum deceleration of node 6851 is higher than the other nodes. This is because this node lies on the right end of the vehicle at the middle, where the front of the vehicle on the same side undergoes an impact. This rapid deceleration causes a bulging of the body structure, and the skin on the right side and this phenomenon is supported by the top view of the vehicle in Figure 5.40. The $\mathrm{Z}$ deceleration of the nodes in the rear of the vehicle is small compared to the front and the middle portion of the vehicle. However, due to this deceleration, the rear body skin undergoes a bulging that can be seen from the top view of the vehicle in Figure 5.40. The acceleration of the nodes in the three figures appear to be oscillating about $0 \mathrm{~m} / \mathrm{sec}^{2}$; thus the bus is approaching steady state. 


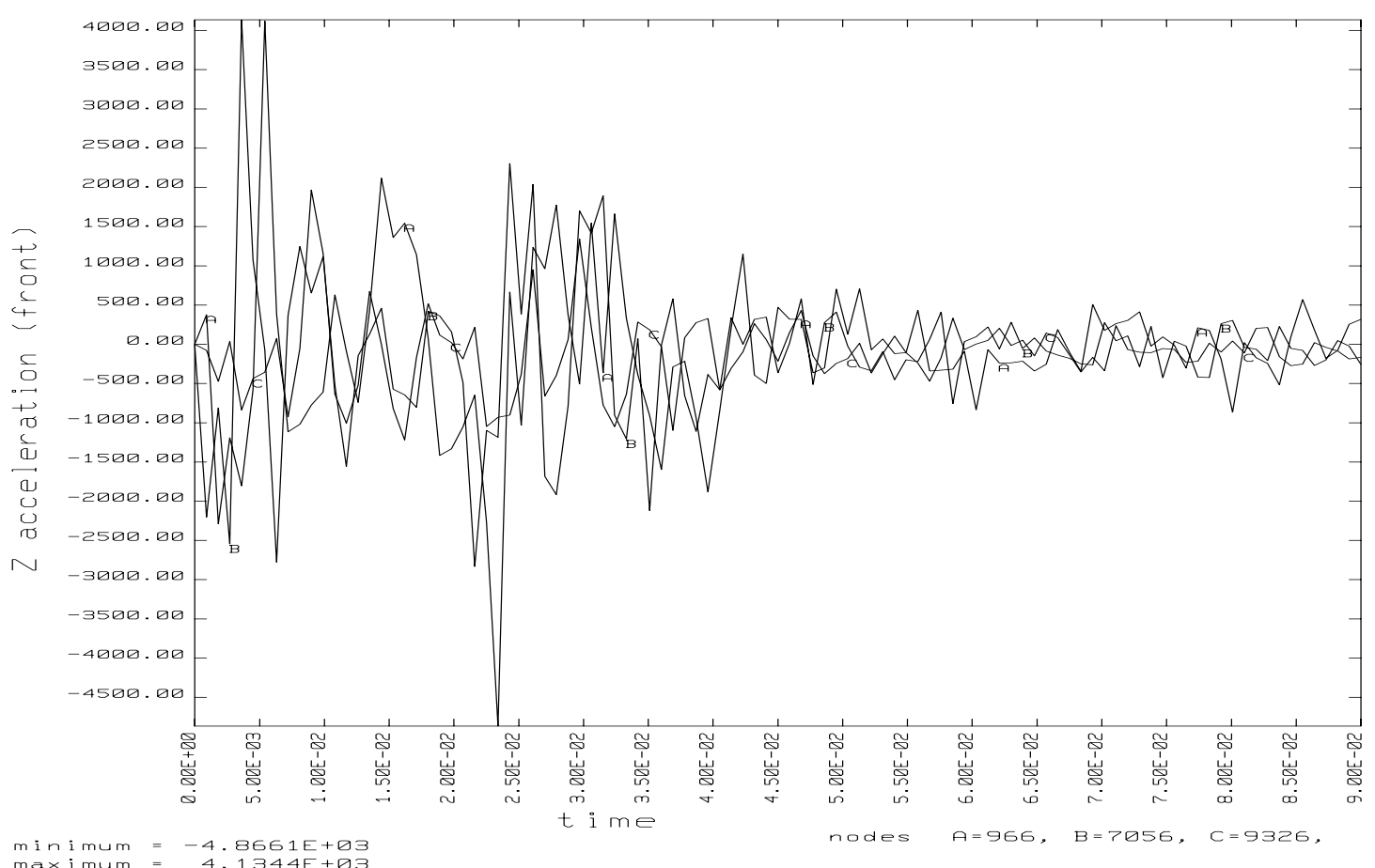

Fig 5.50 $\mathrm{Z}$ acceleration for nodes in the front of the vehicle.

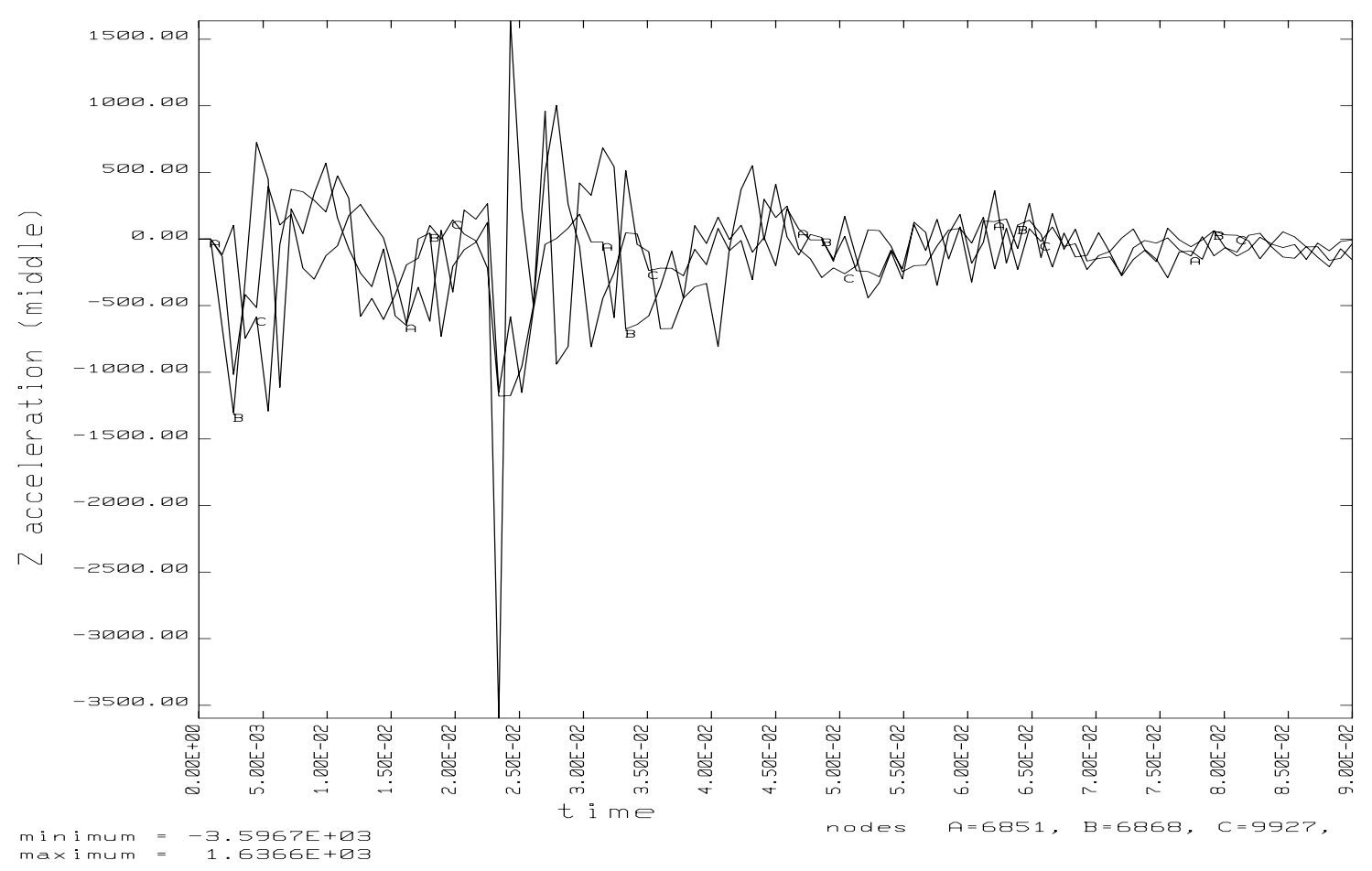

Fig 5.51 $\mathrm{Z}$ acceleration for the nodes in the middle of the vehicle. 


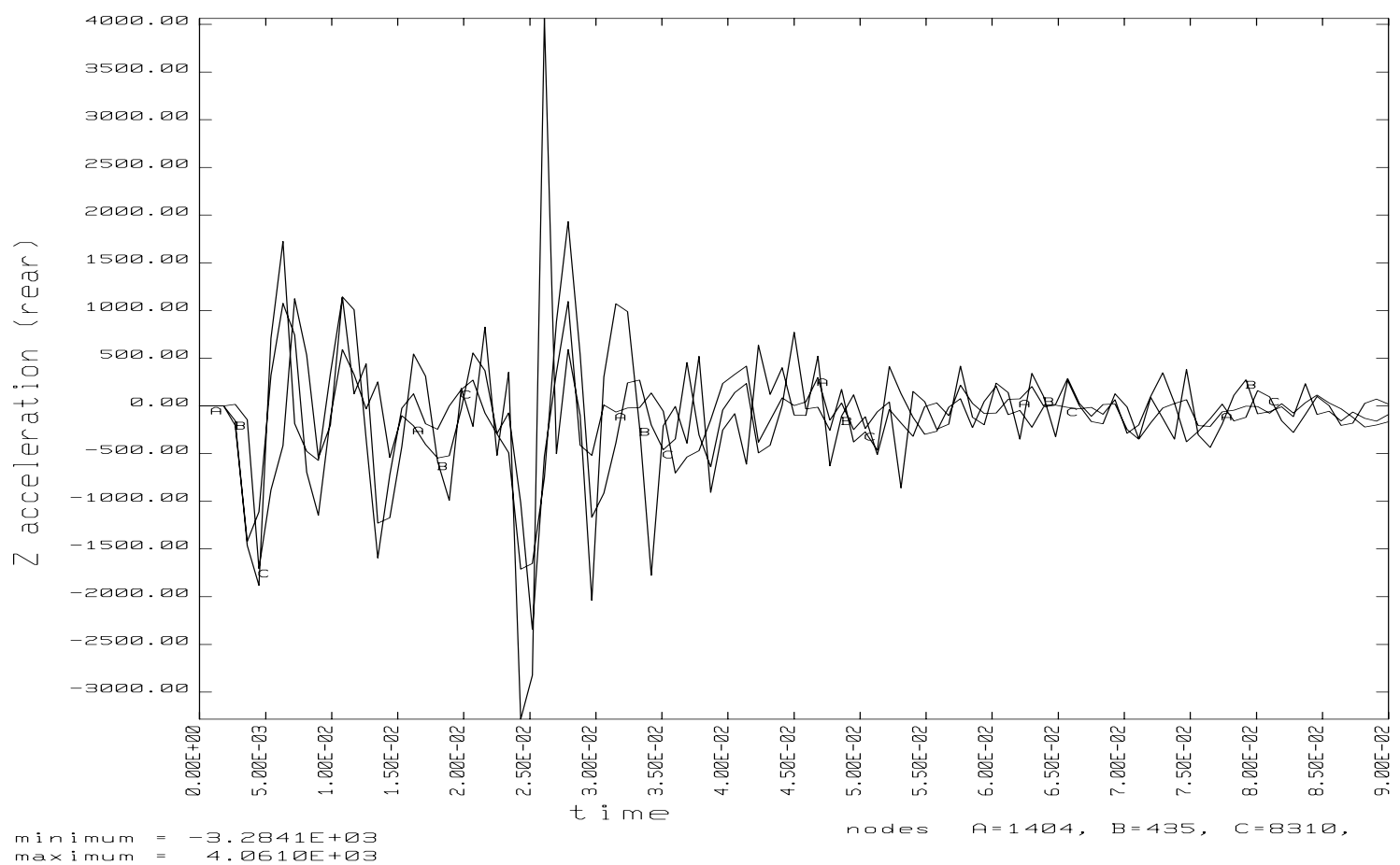

Fig 5.52 $\mathrm{Z}$ acceleration of the nodes in the rear of the vehicle.

Figure 5.50 shows the $\mathrm{Z}$ rigid body velocity for different components of the bus. The rigid body velocity of the radiator decreases rapidly at time $=5 \mathrm{msec}$ which shows that the radiator has started deforming and the rigid body velocity reduces to zero at this time. The rigid body velocity for the hood decreases gradually and becomes zero only at the end of the analysis. The rigid body velocity for the frame lessens gradually, which shows that the final shape of the frame is a combination of the deformation and a rigid body velocity in which the frame slides and rises up against the rigid wall. This can be seen from Figure 5.34. 


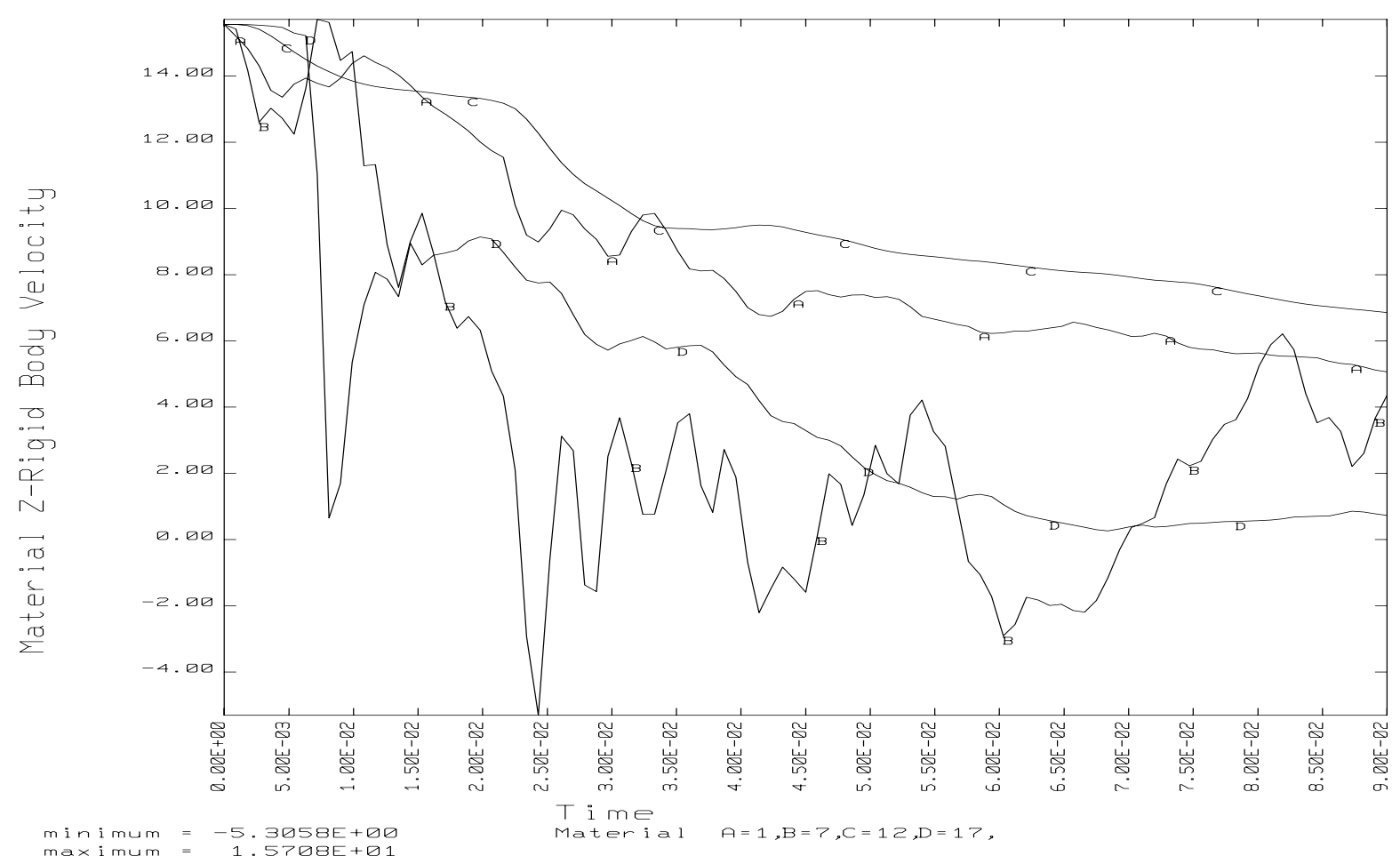

Fig 5.53 $\mathrm{Z}$ rigid body velocity for materials (Refer to Table 5.1 for table of materials).

The Figure 5.54 shows the internal energy absorbed by various components. The internal energy absorbed by the frame (Material 1) is the maximum. The radiator (Material 2) absorbs maximum energy of the system next to the frame. This is supported by the pie chart shown in Figure 5.56, which shows that the hood absorbs less energy compared to the frame, even though it deforms in shape. The body structure (Material 12) absorbs one percent of the total internal energy, which shows that fewer impact forces are transmitted to the occupant area. 


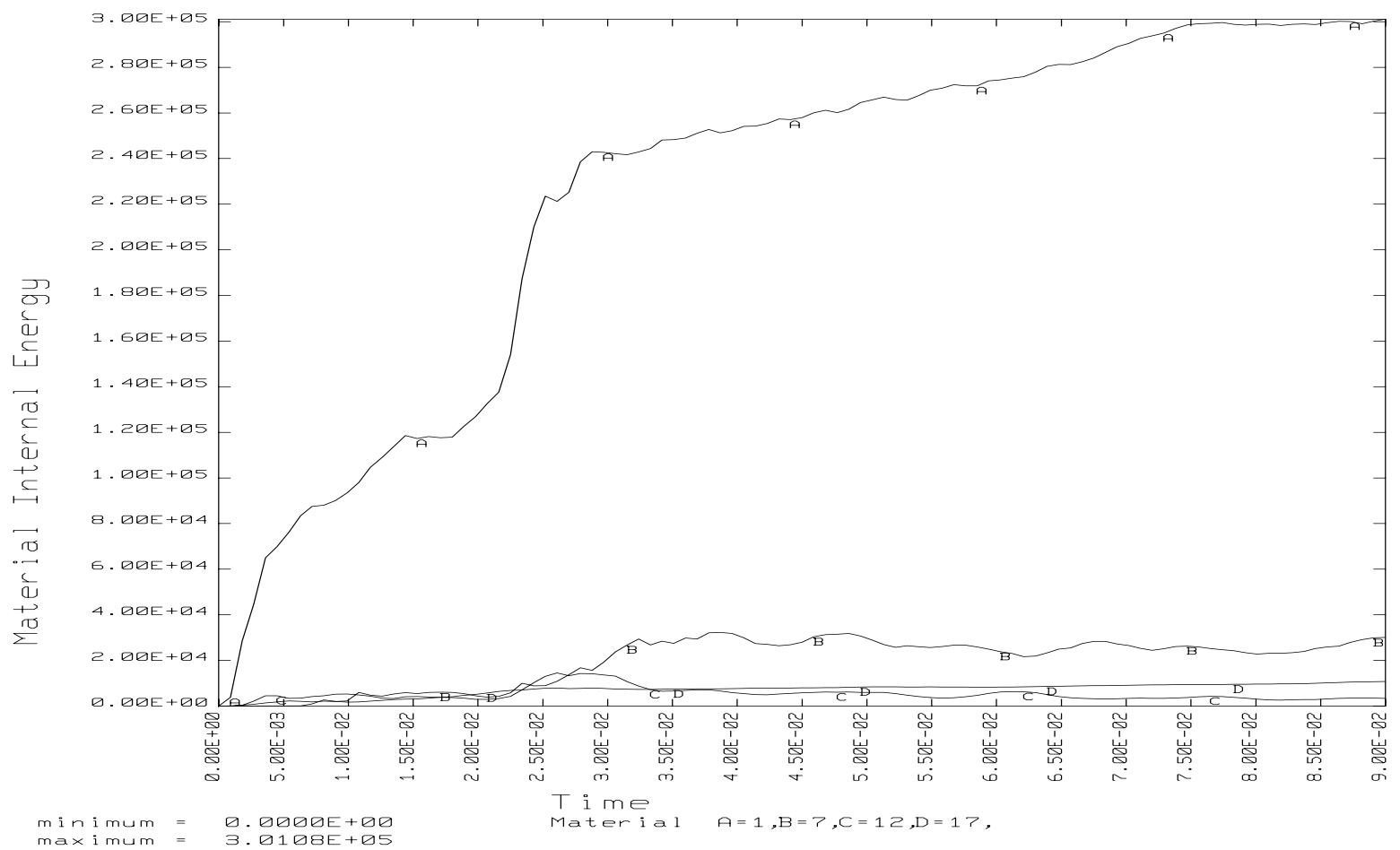

Fig 5.54 Internal energy absorbed by materials (Refer to Table 5.1 for table of materials).

The graph in Figure 5.55 shows the internal, kinetic, and the total energies of the system. The total energy of the system remains as a constant, which shows that the energy is conserved during the analysis. The kinetic energy lessens as the vehicle decelerates and is converted to internal energy of the system. Compared to Figure 5.29 for a full frontal impact, in the offset frontal impact, the kinetic energy is converted into internal energy at a slower rate than the full frontal impact. The sum of the internal and the kinetic energy is not equal to the total energy because of the presence of hourglass energy in the thin shell elements. Since full integration was done for the solid elements, there is no hourglass energy present in the solid elements. 


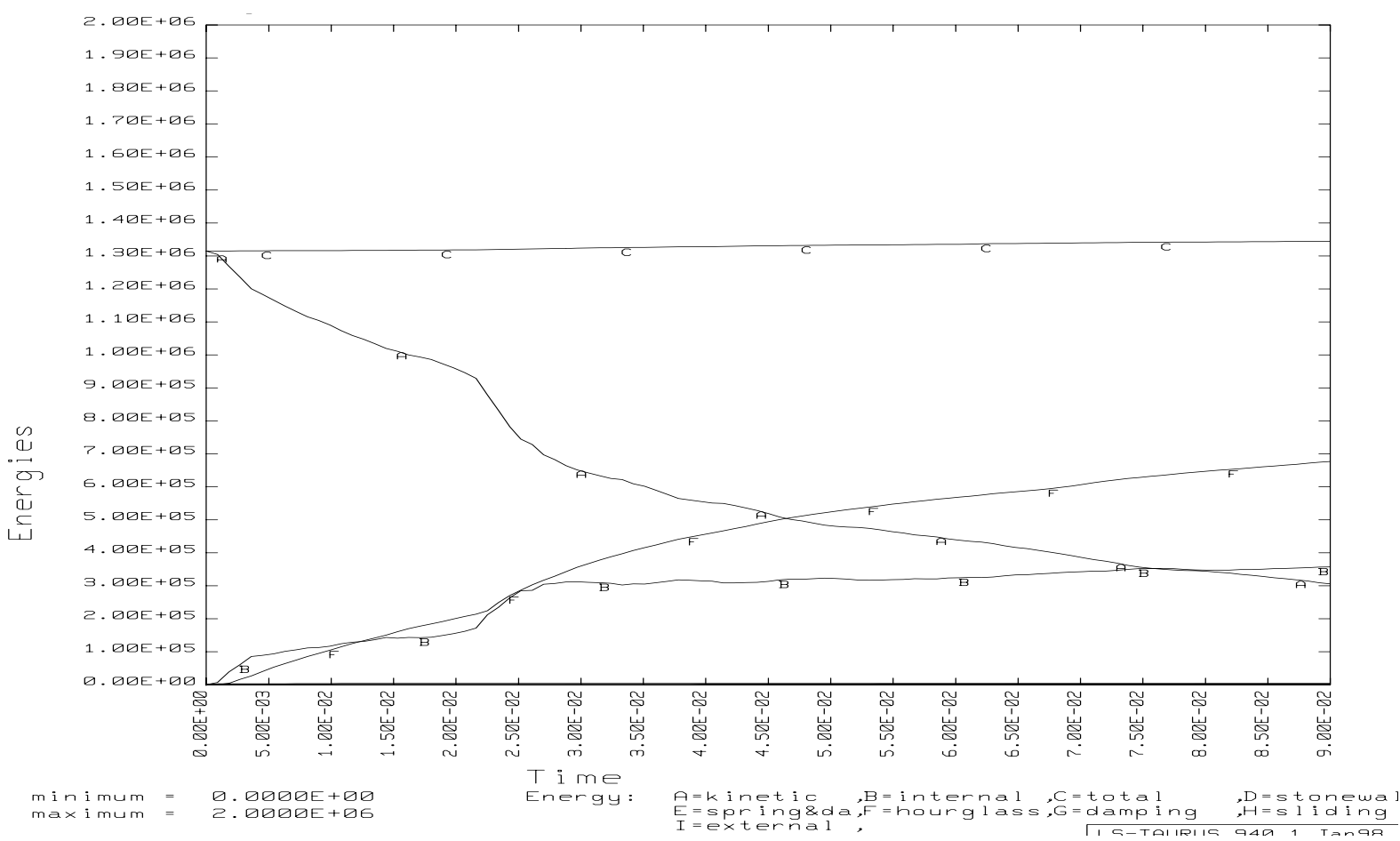

Fig 5.55 Kinetic, internal and the total energies for an offset frontal impact.

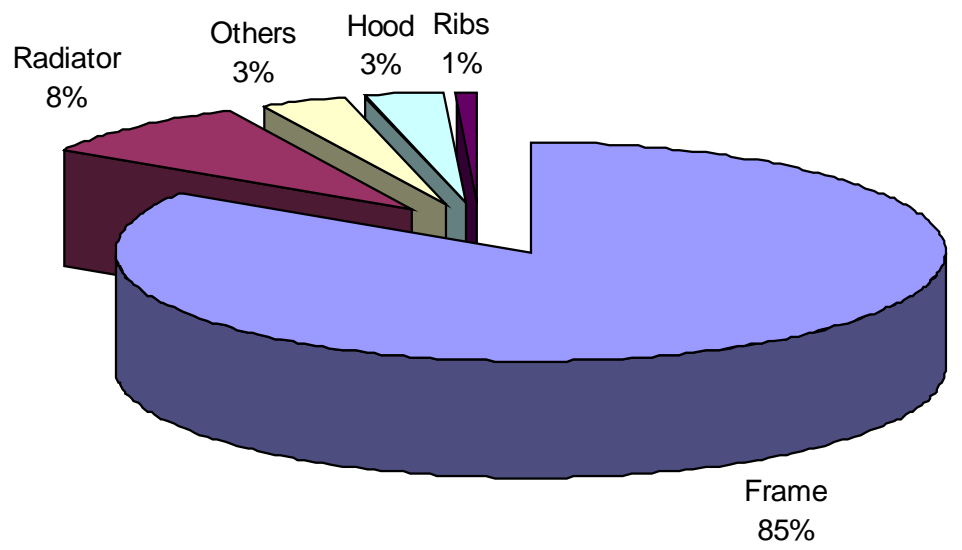

Fig 5.56 Total internal energy distribution at time $=90$ msec of the bus subjected to an offset frontal impact.

\subsection{Higher ORdER INTEGRATION FOR THIN SHELL ELEMENTS}

The hourglassing energy for the thin shell elements was high for the analysis run before and it was equal to the magnitude of the kinetic energy. To avoid the hourglass energy for the thin shell elements, Hughes-Liu elements with Selective-Reduced 
integration have to be used. Since these elements are integrated using 2 X 2 Gaussian quadrature in the plane, these elements are recommended in areas where zero energy modes (hour glassing) is a problem. Using these elements, the run time for the analysis is increased by five times the previous analyse's. So the analysis was terminated as soon as the solution reached a steady state, (i.e.) when the velocity became constant or the acceleration became zero. A comparison is made between the analyses with and without hour glassing for a full frontal impact to find the differences in the energy management, deformation, displacement, velocity, and acceleration.

The Hughes-Liu elements with selective reduced (SR) integration were used for all the thin shell elements in the model. No changes were made in the mesh density and the total number of elements in each part remained the same. The initial and the boundary conditions remained the same for the analyses. The hourglassing energy remained zero through out the analysis because eight-point integration was done for the solid elements and Hughes-Liu element formulation was used for the thin shell elements. The total energy remained as a constant, which was equal to the sum of the kinetic and the internal energies at all time states.

\subsection{Full Frontal Impact With Hughes-Liu SR Thin SHELl ElementS}

Figure 5.57 shows the front view of the vehicle at time $=0 \mathrm{msec}$. The initial conditions and the boundary conditions were the same for both analyses run with the single point integration and the Hughes-Liu SR thin shell elements. The only difference between the two analyses is the element formulation for the thin shell elements. 


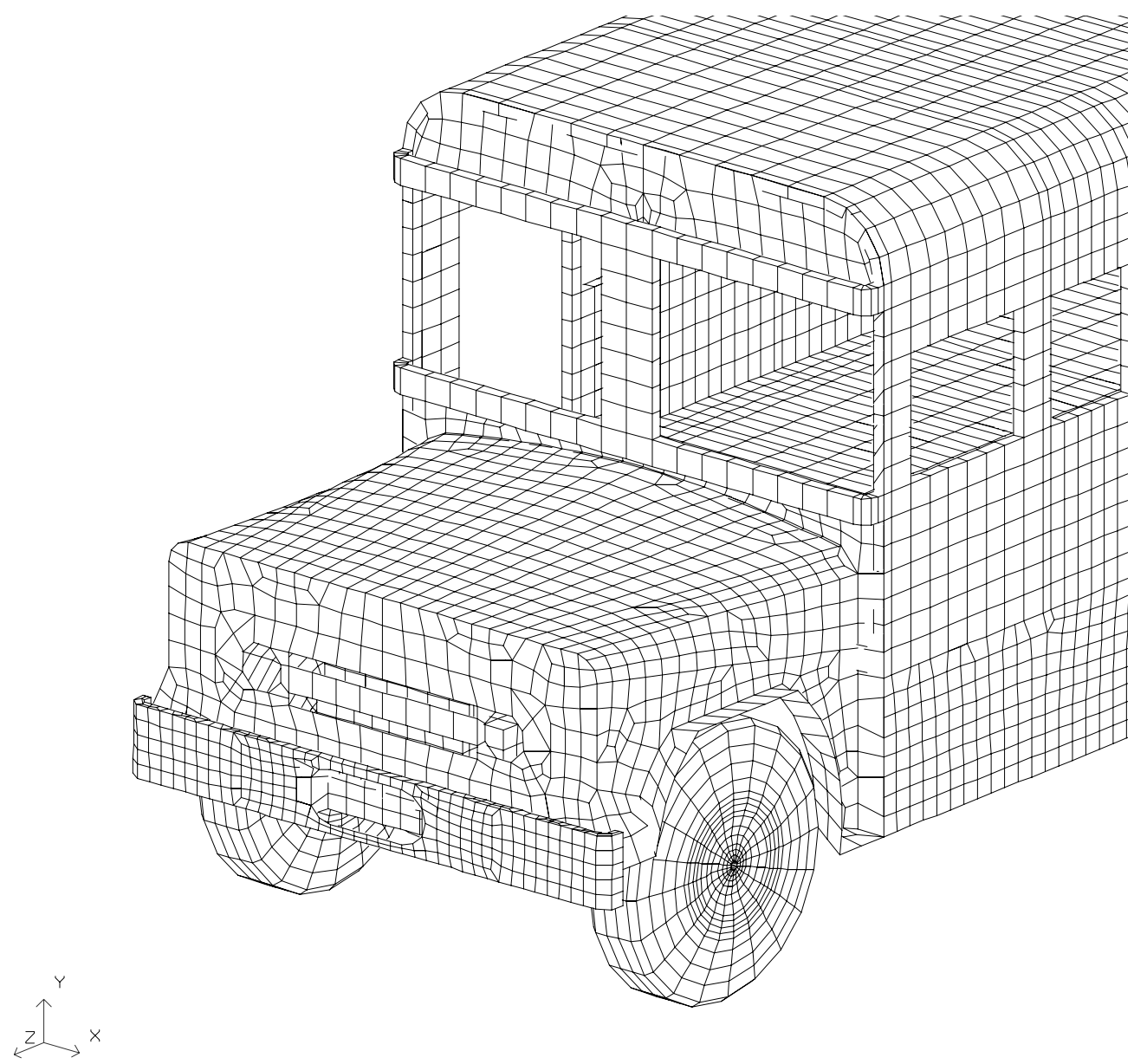

Fig 5.57 Front view at time $=0$ msec of the bus subjected to a full frontal impact with Hughes-Liu SR thin shells.

Figure 5.58 shows the front view of the bus at time $=9 \mathrm{msec}$. The bumper hits the rigid wall at this time state and the deformation on the bumper can be seen in the figure. The kinetic energy of the vehicle is converted to internal energy and is absorbed by the components in the front end of the vehicle. The kinetic energy decreases due to the deceleration of the vehicle and the internal energy increases. This can be seen from the graph for the global energy plotted in Figure 5.86. The rigid wall force reaches a maximum of $9.8 \mathrm{MN}$ at time $=4 \mathrm{msec}$ as compared to the rigid wall force of $8 \mathrm{MN}$ in a single point integration run. The internal energy of the vehicle is absorbed only by the frame, which can be seen in Figure 5.85. 


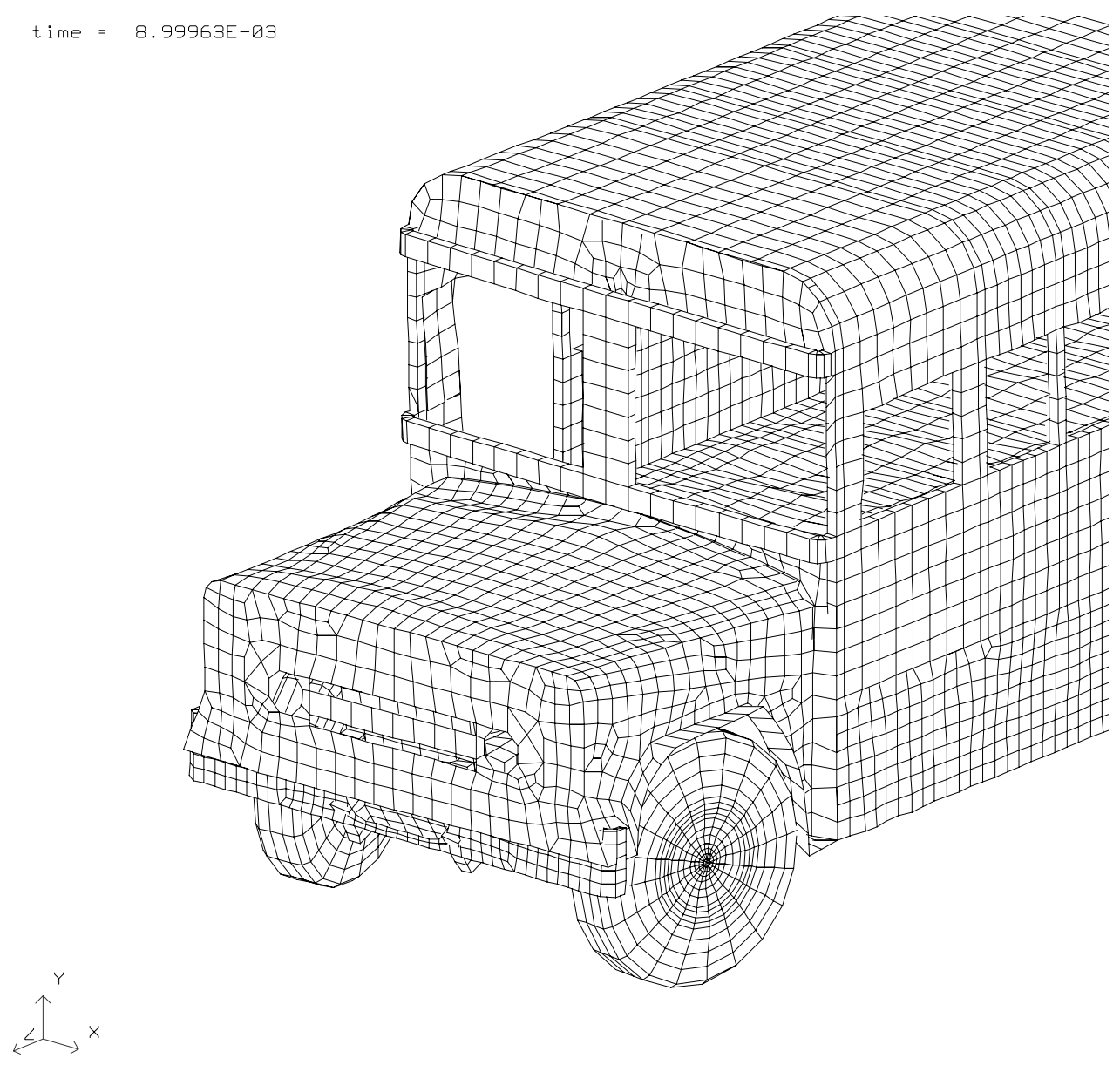

Fig 5.58 Front view at time $=9$ msec of the bus subjected to a full frontal impact with Hughes-Liu SR thin shells.

Figure 5.59 shows the front view of the bus at time $=18 \mathrm{msec}$. The hood has impacted the rigid wall by this time and the deformation of the hood can be seen in the figure. The kinetic energy of the vehicle decreases rapidly due to the deceleration of the vehicle and the internal energy increases as seen from Figure 5.86. When the $\mathrm{Z}$ relative displacement between the frame and the front tires is compared as seen in Figure 5.67, it can be seen that the relative displacement increases in the positive direction, which shows that both the parts are moving in the opposite direction, unlike the relative displacement in the case of a single point thin shell element formulation as seen in Figure 5.15. The stiffness of the frame is high and the frame bends without any compression in the higher 
order element formulation. But in the case of a single point formulation for thin shells, the frame compresses without bending.

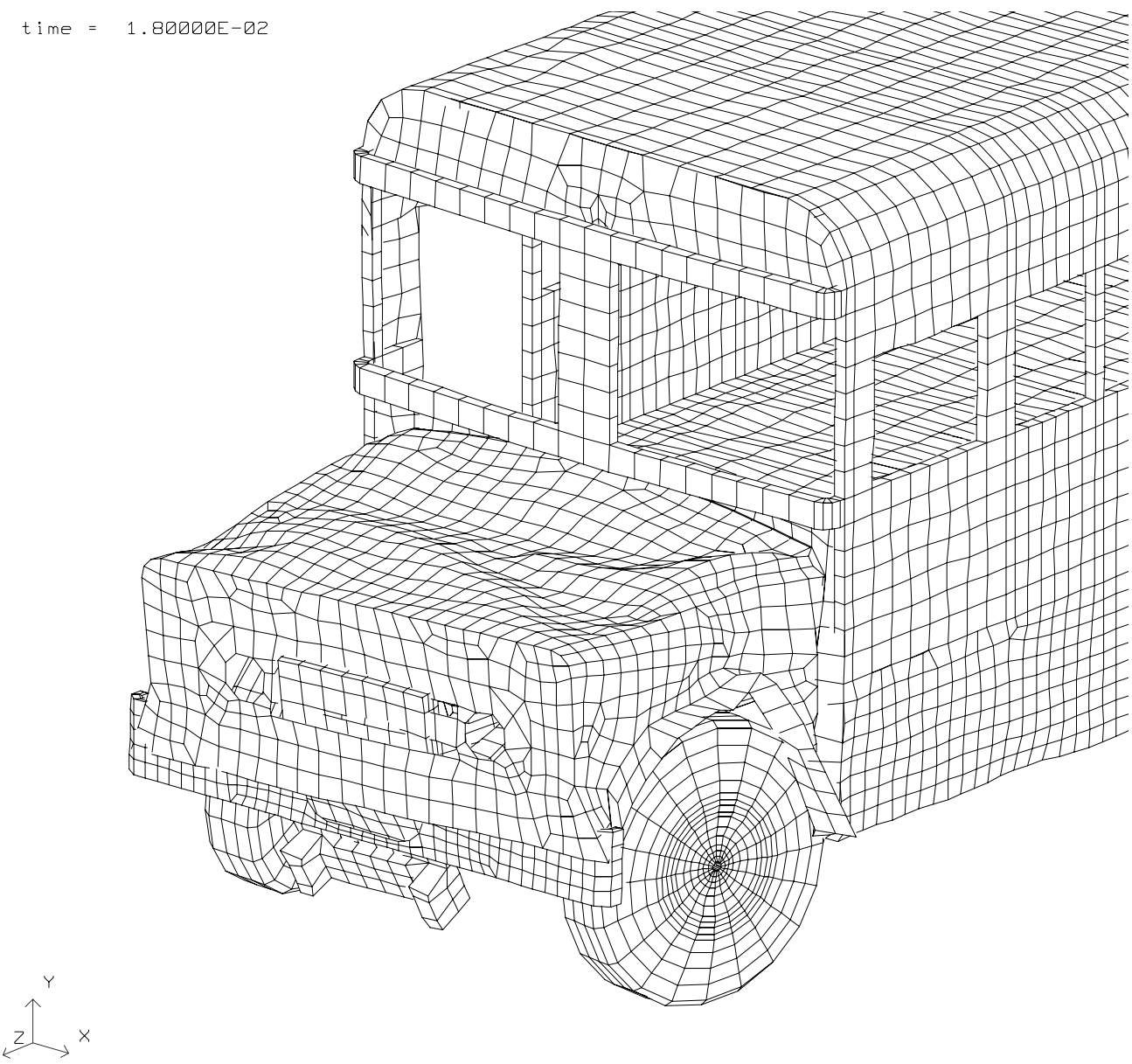

Fig 5.59 Front view at time $=18$ msec of the bus subjected to a full frontal impact with Hughes-Liu SR thin shells.

Figure 5.60 shows the front view of the bus at time $=27 \mathrm{msec}$. The radiator and the front cross member hit the rigid wall and the maximum energy is absorbed by the frame. The internal energy absorbed by the frame reaches a maximum at time $=19 \mathrm{msec}$, and the maximum energy absorbed by the frame is 5,24,000 joules as compared to $3,20,000$ joules for the single point formulation. The maximum stress in the fire wall is higher than the yield stress of the material of the fire wall and hence the deformation is plastic on the 
fire wall. The maximum absolute displacement of the fire wall at this time state is $0.23 \mathrm{~m}$ as compared to $0.3 \mathrm{~m}$ for the single point element formulation.

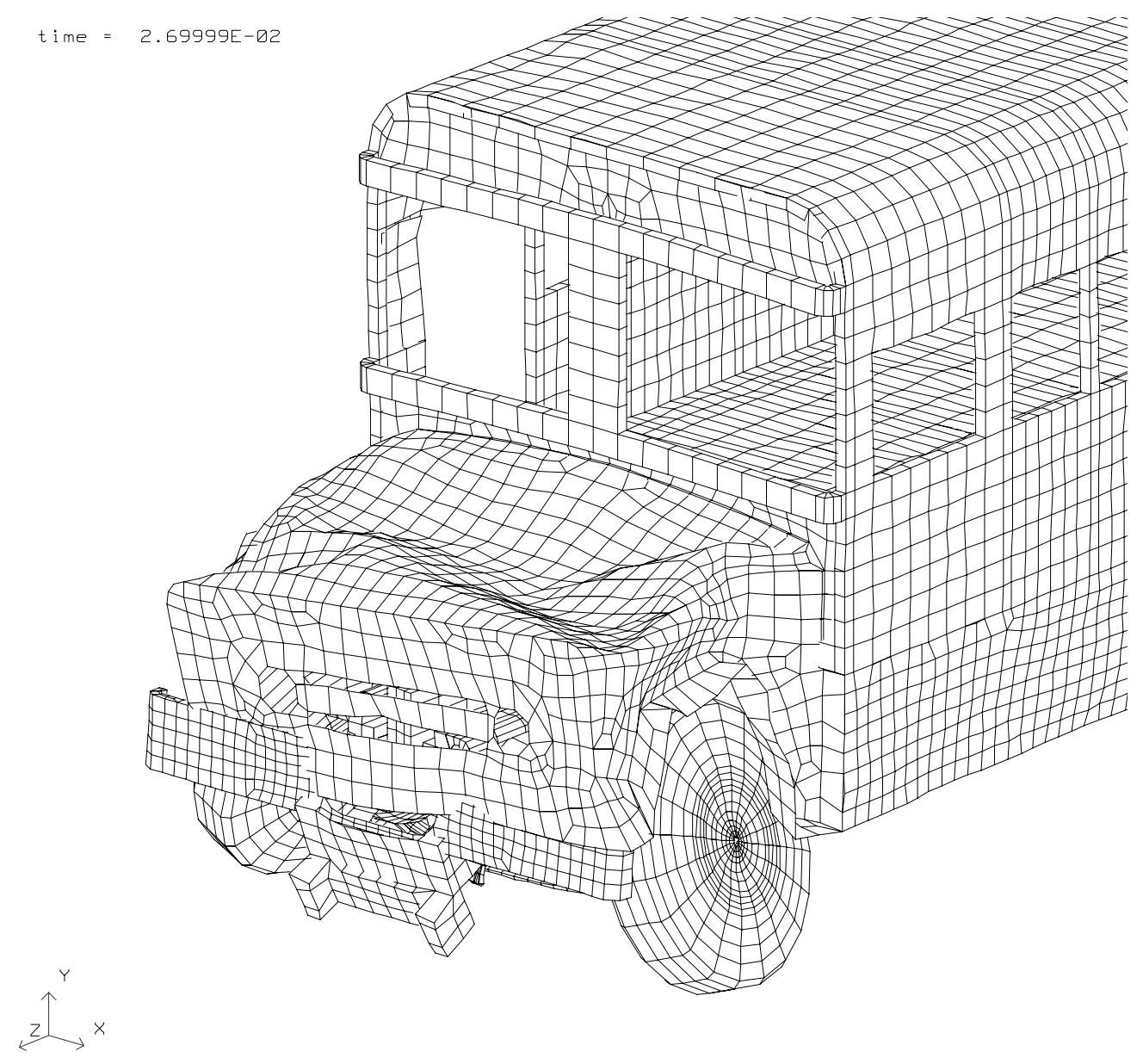

Fig 5.60 Front view at time $=27$ msec of the bus subjected to a full frontal impact with Hughes-Liu SR thin shells.

Figure 5.61 shows the front view at time $=36 \mathrm{msec}$ of the bus subjected to a full frontal impact with Hughes-Liu SR thin shell elements. The relative displacement between the front end of the frame and the tires increases as seen in Figure 5.67, showing that the tip of the frame and the tires move apart. The relative displacement between a node on the hood and a node on the front end of the frame increases as seen in Figure 5.66, which shows that the frame moves in the negative $\mathrm{Y}$ direction and the hood moves 
in the positive $\mathrm{Y}$ direction. The maximum absolute displacement in the fire wall is 0.206 m compared to $0.308 \mathrm{~m}$ in the full frontal analysis without Hughes-Liu SR thin shells. But in any case there is no intrusion into the occupant area.

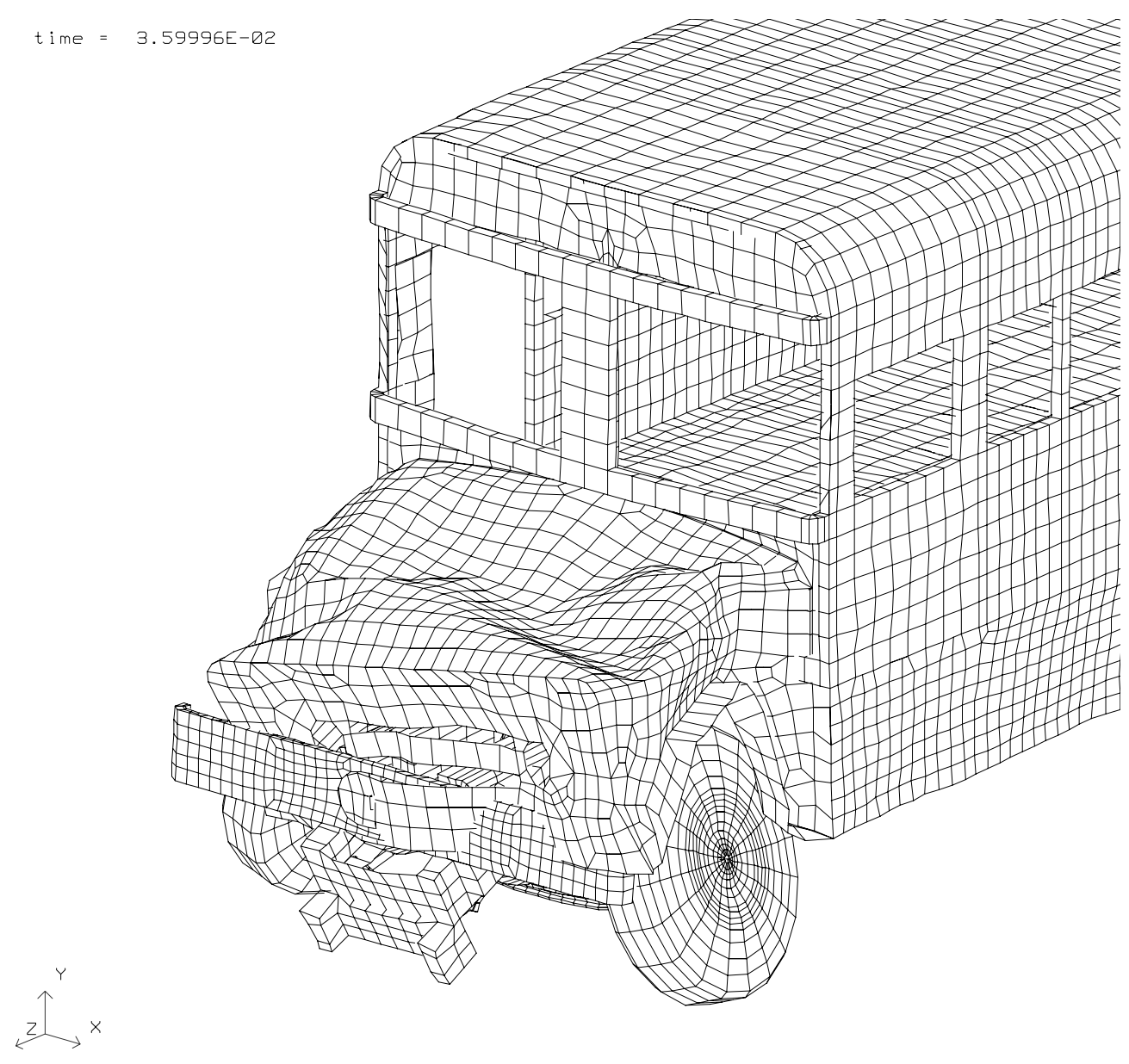

Fig 5.61 Front view at time $=\mathbf{3 6}$ msec of the bus subjected to a full frontal impact with Hughes-Liu SR thin shells.

Figure 5.62 shows the picture of the chassis of the bus at time $=45 \mathrm{msec}$ subjected to a full frontal impact with Hughes-Liu SR thin shells. The buckling of the frame after the second engine mounting cross member can bee seen in the figure. The frame absorbs the maximum internal energy, and this case is the same as the full frontal impact without higher order integration for thins shells. However the behavior of the frame is different in 
both the cases and the frame is stiffer and does not undergo any compression as in the case of full frontal impact without higher order integration for thin shells. The front end of the frame moves up in the case of a full frontal impact without Hughes-Liu thin shells, but the front end of the frame moves down in the case of a full frontal impact with Hughes-Liu thin shells. The bending of the propeller shaft is illustrated and the bending may be higher because of the absence of the bolts that clamp the propeller shaft to the frame. The global velocity of the system reaches a constant value at this time, which shows that the acceleration of the bus oscillates about $0 \mathrm{~m} / \mathrm{s}^{2}$ and the velocity of the system can be seen in Figure 5.84.

time $=4.50000 \mathrm{E}-02$
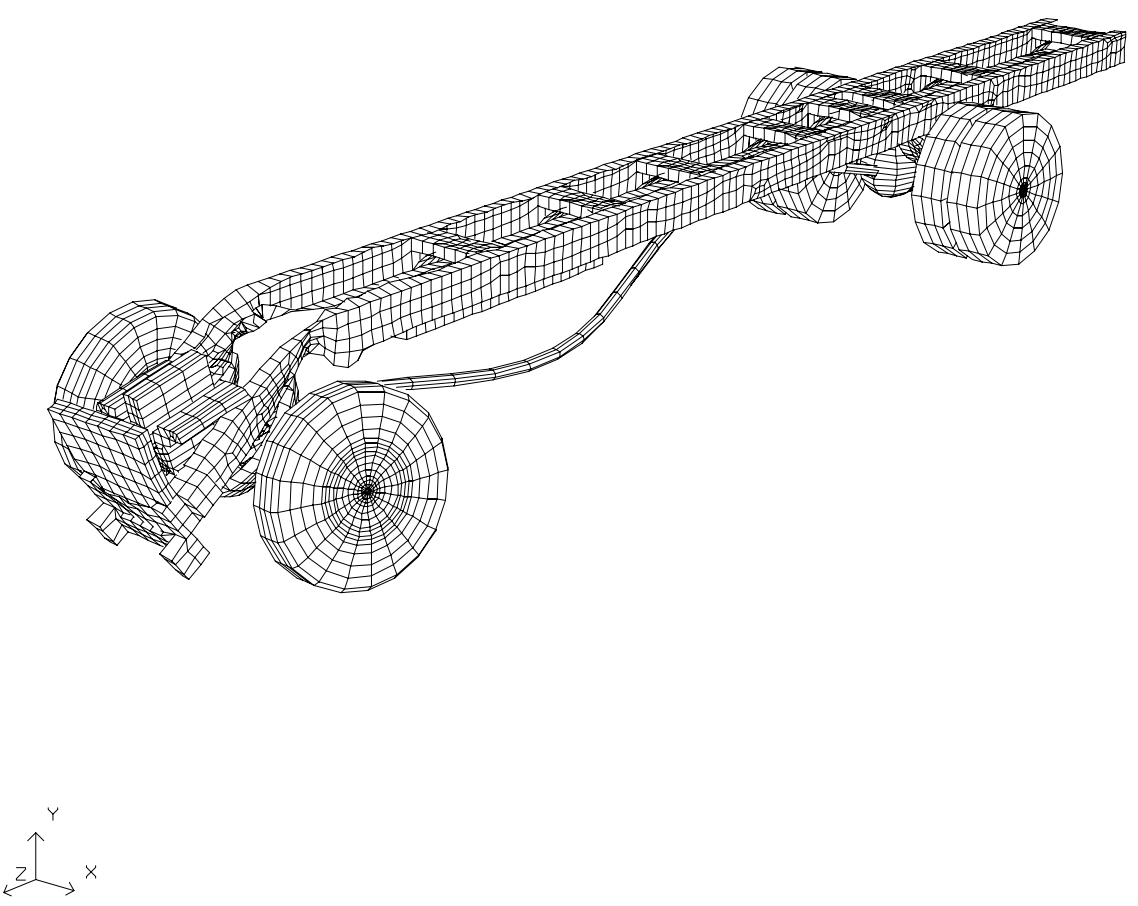

Fig 5.62 Front view at time $=45$ msec of the chassis of the bus subjected to a full frontal impact with HughesLiu SR thin shells. 
Figure 5.63 shows the VonMises stress plot for the frame of the vehicle subjected to a full frontal impact with Hughes-Liu SR thin shells. The maximum stress in the frame is $18900 \mathrm{MN} / \mathrm{m}^{2}$, which is higher than the yield stress of the material of the frame, and this can be seen in Appendix C. The maximum stress for a full frontal impact with HughesLiu SR thin shells is higher than the maximum stress that occurs in the frame without Hughes-Liu SR thin shells as seen in Figures 5.14 and 5.63. However, in both the cases the maximum stress is higher than the yield stress of the material of the frame. In the case of a full frontal impact without Hughes-Liu SR thin shells, the front end of the frame rises up as it hits the rigid wall, which can be seen in Figure 5.14, but in the case of a full frontal impact with Hughes-Liu SR thin shells the front end of the frame dips down as seen in Figure 5.63. This behavior of the frame in the first case is due to the presence of hourglassing in the numerical solution. This is more evident by seeing the deformation of the front end of the frame in Figure 5.14. The front cross member and the two engine mounting cross members have also failed at this time. The stresses were plotted for the shell middle surface for both the cases. 


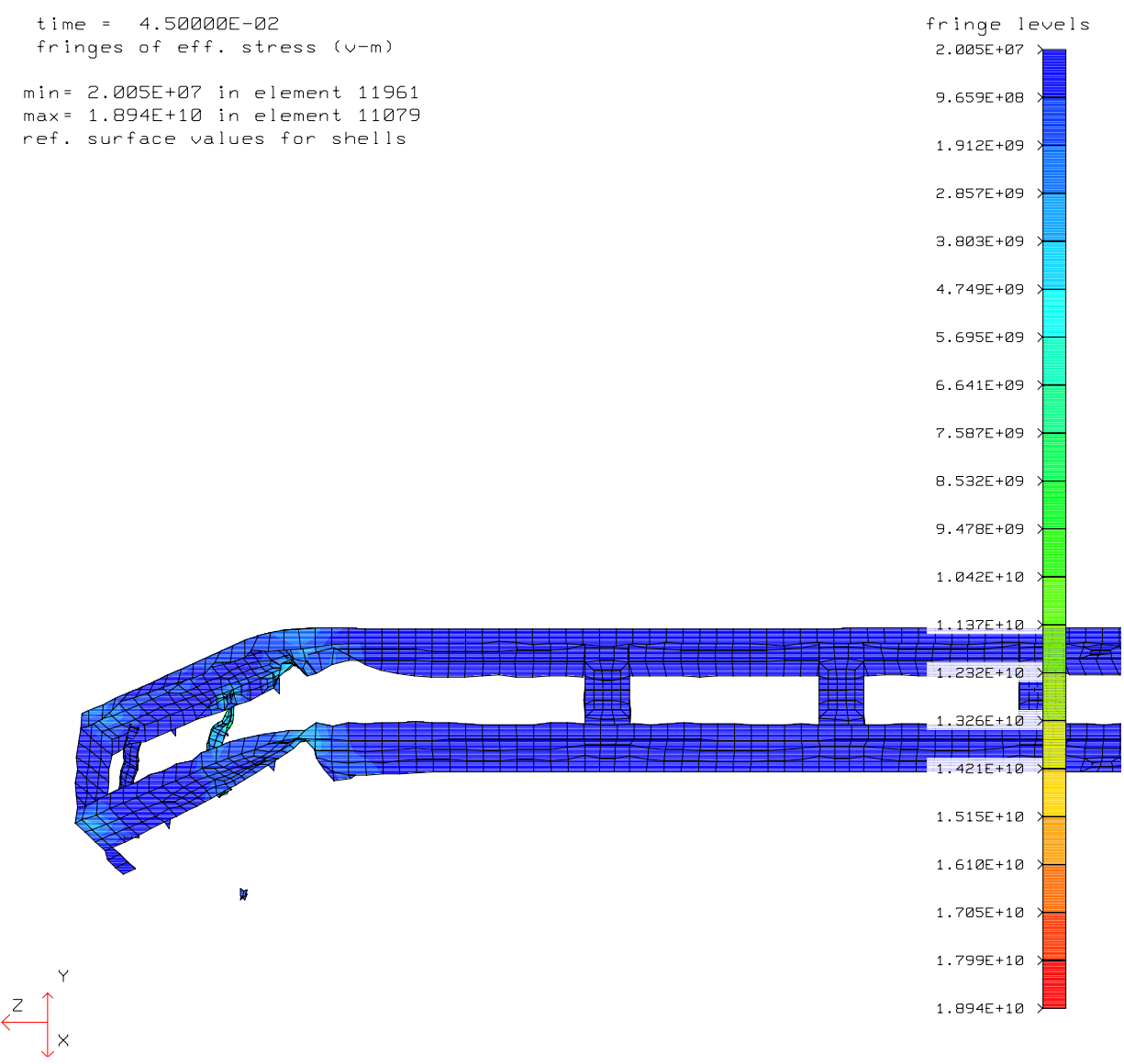

Fig 5.63 VonMises stress plot in the front end of the frame at time $=45 \mathrm{msec}$ for a full frontal impact with Hughes-Liu SR thin shells.

Figures 5.64 and 5.65 show the side view and the top view at time $=45 \mathrm{msec}$ of the bus subjected to a full frontal impact with Hughes-Liu SR thin shells. The side view of the bus shows the bending of the frame, and the front end of the frame moves down as it hits the rigid wall. The body structure of the bus remains horizontal level and only the frame moves down in the case of a full frontal impact with Hughes-Liu SR thin shells as illustrated. But in the case of a full frontal impact without Hughes-Liu SR thin shells, the frame moves up and the nose of the bus dips down as seen in Figure 5.11. The side view of the bus shows that the bulging of the body skin at the rear end is the same in both analyses for a full frontal impact, and this can be seen in Figures 5.12 and 5.65. However, 
the deformation in the hood is higher in the case of a full frontal impact without HughesLiu SR thin shells because of the presence of the hourglassing energy in the system, which causes an exaggerated deformation in the system.

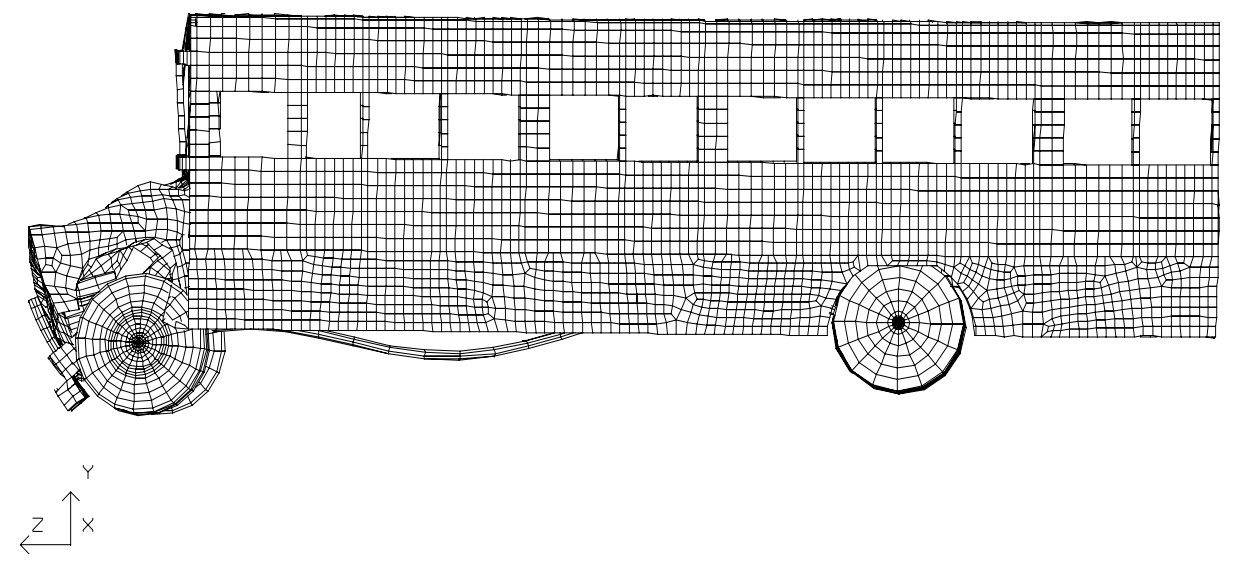

Fig 5.64 Side view at time $=\mathbf{4 5}$ msec of the bus subjected to a full frontal impact with Hughes-Liu SR thin shells.
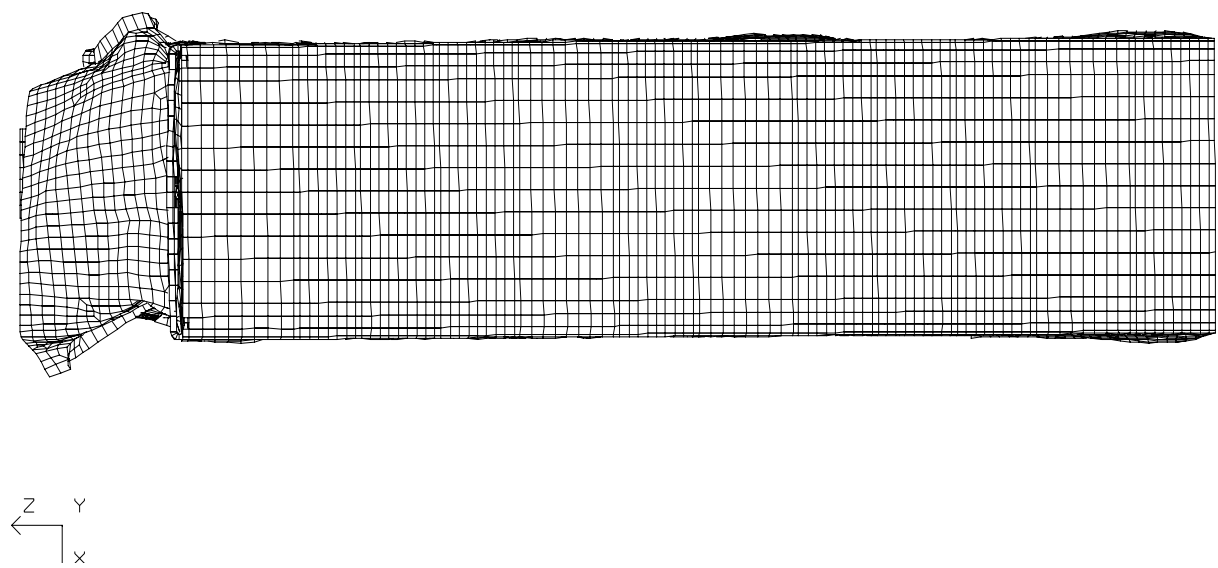

Fig 5.65 Top view at time $=\mathbf{4 5}$ msec of the bus subjected to a full frontal impact with Hughes-Liu SR thin shells. 
Figure 5.66 shows the Y relative displacement between the nodes in the hood and the frame. The relative displacement increases in the positive Y direction, which shows that the node 26 on the hood moves at a slower rate in the Y direction and node 16017 on the front end of the frame moves at a higher rate in the negative $\mathrm{Y}$ direction. This behavior of the vehicle is supported by the picture of the vehicle showing its side view in Figure 5.64. The $\mathrm{Y}$ relative displacement increases up to $32.5 \mathrm{msec}$ after which it decreases.

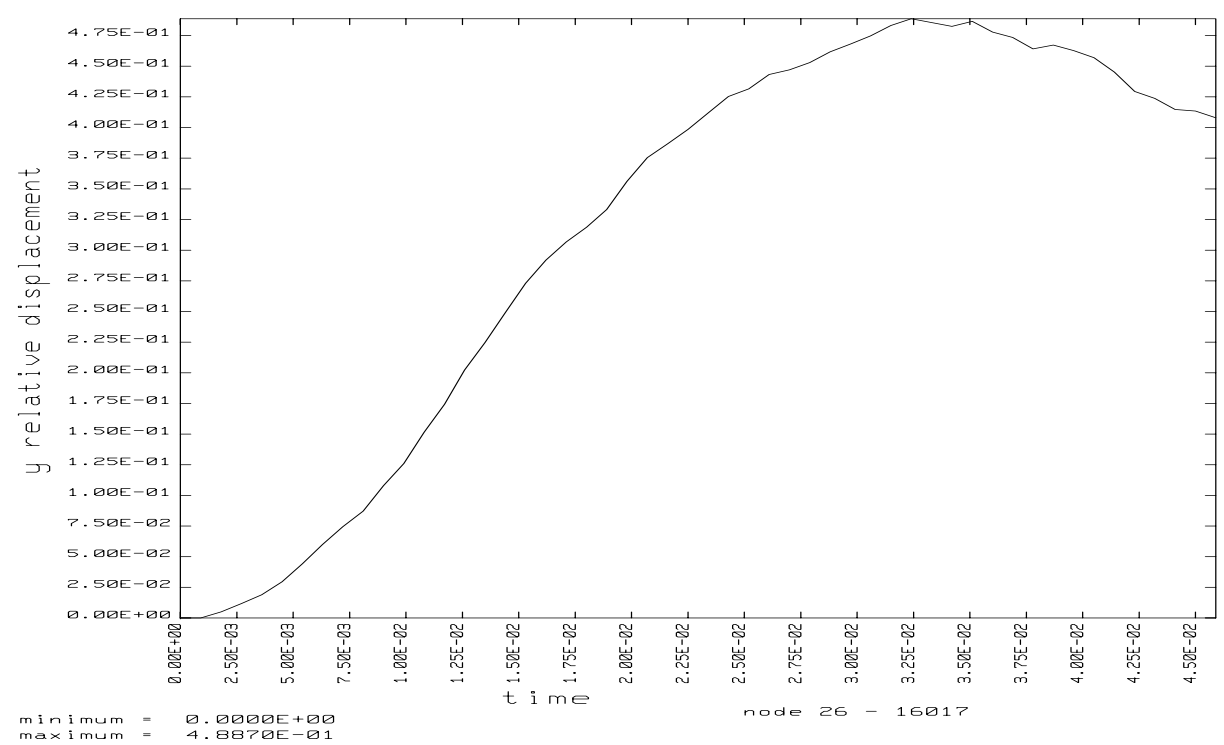

Fig 5.66 Relative displacement between the hood and the frame for a full frontal impact with Hughes-Liu SR thin shells.

Figure 5.67 shows the $\mathrm{Z}$ relative displacement between the nodes in the frame and the front tire for a full frontal impact with Hughes-Liu SR thin shells. The Z relative displacement is positive and increases, whereas in the case of a full frontal impact without Hughes-Liu SR thin shells the relative displacement is negative. This shows that in this case the front end of the frame and the front tire move apart in the $\mathrm{Z}$ direction where as in the case of the full frontal impact without Hughes-Liu SR thin shells, they 
move closer in the positive $\mathrm{Z}$ direction. This is due to the difference in the behavior of the frame.

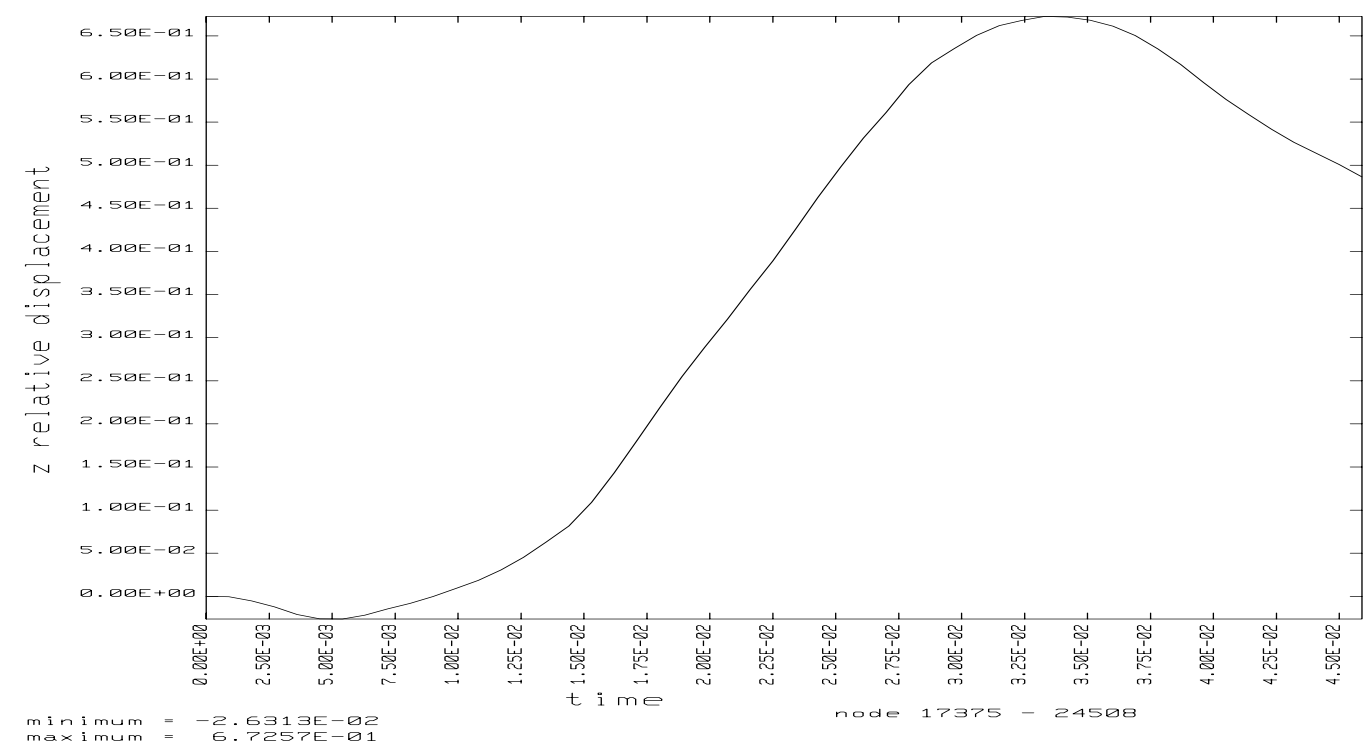

Fig 5.67 $\mathrm{Z}$ relative displacement between the frame and the front tire for a full frontal impact with Hughes-Liu SR thin shells.

Figures 5.68, 5.69, and 5.70 show the $\mathrm{Z}$ displacement of the nodes in the front, middle, and rear of the vehicle for a full frontal impact. The $\mathrm{Z}$ displacement in the front and the middle of the vehicle is the same, which shows that there is no deformation in the body structure up to the middle of the bus. The displacement of the nodes in the rear of the vehicle is higher than the front and the middle of the vehicle, which shows that there is a bulge in the body structure between the middle and the rear of the vehicle. There is no protrusion in the occupant area since the difference in the deformation levels are less. This phenomenon is supported by the picture of the top view of the bus in Figure 5.65. This behavior of the vehicle is the same as in the full frontal impact without Hughes-Liu SR thin shells, but the maximum displacement of the nodes is higher in the case without Hughes-Liu SR thin shells. The nodes in the left, center, and left side of the vehicle have 
the same displacement in the middle and the rear of the vehicle, but there is a small difference for the nodes in the front of the vehicle and this can be seen in Figure 5.68.

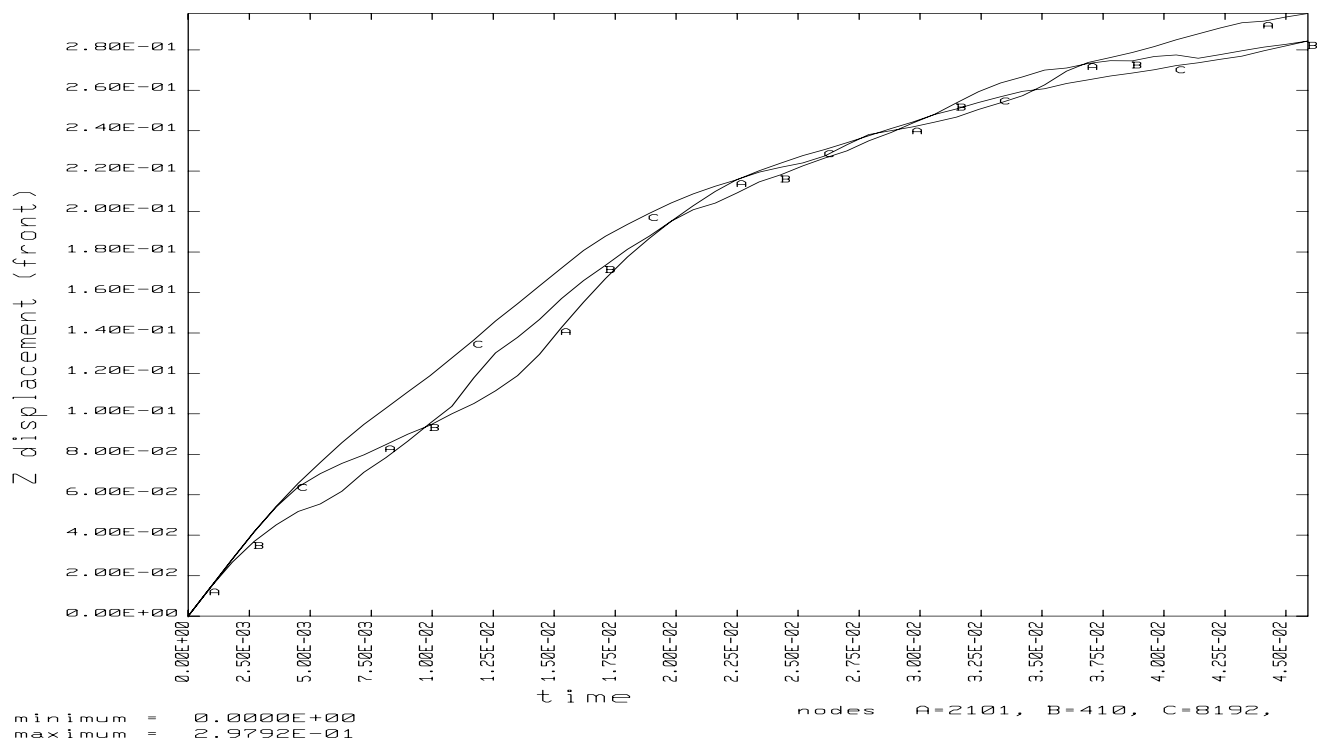

Fig 5.68 $\mathrm{Z}$ displacement for the nodes in the front of the vehicle for full frontal impact with Hughes-Liu SR thin shells.

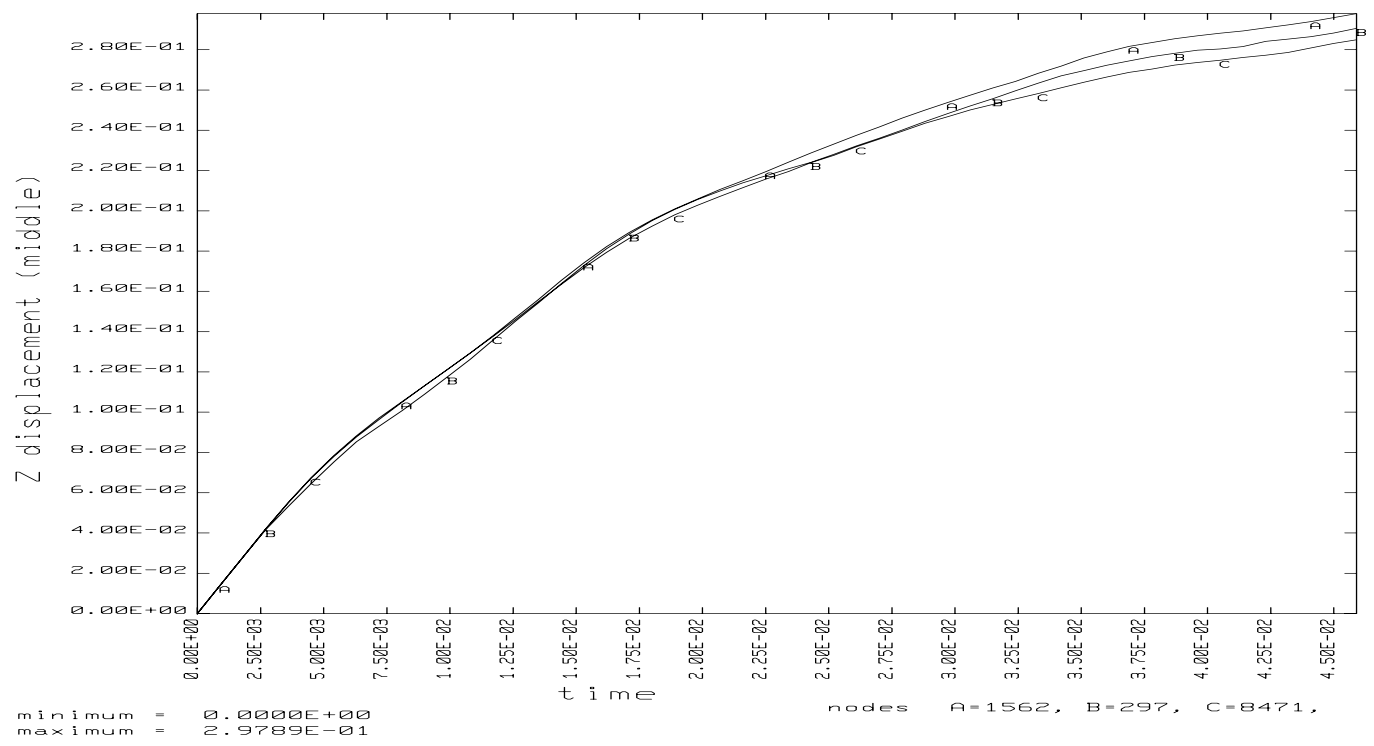

Fig 5.69 $\mathrm{Z}$ displacement for the nodes in the middle of the vehicle for full frontal impact with Hughes-Liu SR thin shells. 


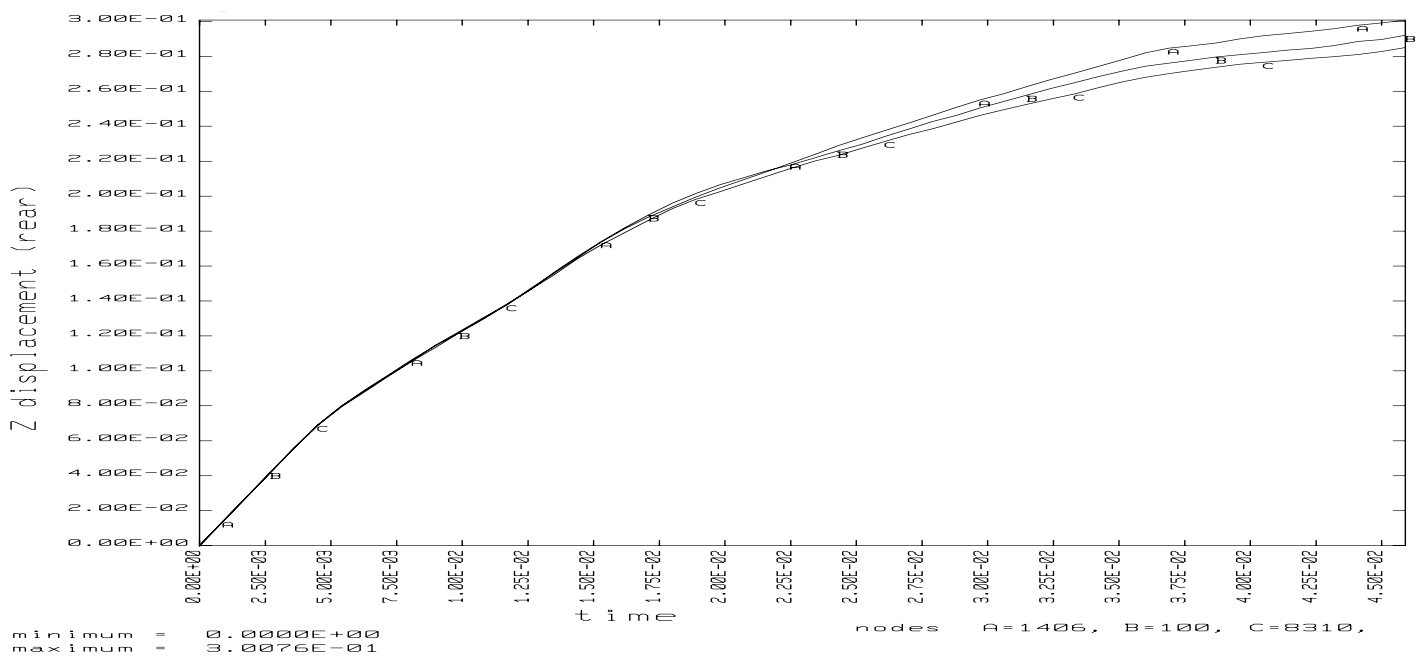

Fig 5.70 $\mathrm{Z}$ displacement for the nodes in the rear of the vehicle for full frontal impact with Hughes-Liu SR thin shells.

Figures 5.71, 5.72, and 5.73 show the $\mathrm{Z}$ velocity for the nodes in the front, middle, and rear of the vehicle. From the figures it can be seen that the velocities for the nodes in the front, middle, and rear of the vehicle are nearly the same. This shows that there is no deformation in the occupant area. The nodes in the middle and the rear of the vehicle behave in the same pattern, whereas the nodes in the front of the vehicle show a difference in values between the left, center, and right of the vehicle. This phenomena is also supported by the difference in the displacement of the nodes in the front of the vehicle as seen in Figure 5.68. The velocity of the nodes in the rear of the vehicle starts to decrease only after $3 \mathrm{msec}$, where as the velocity of the nodes in the front and the middle of the vehicle starts to decrease before $3 \mathrm{msec}$. This is causes the difference in the deceleration levels for the nodes in the front, middle, and rear of the vehicle. The minimum velocity is negative in this case for the nodes in all the three positions of the vehicle as compared to positive values for a full frontal impact without Hughes-Liu SR thin shell elements. 


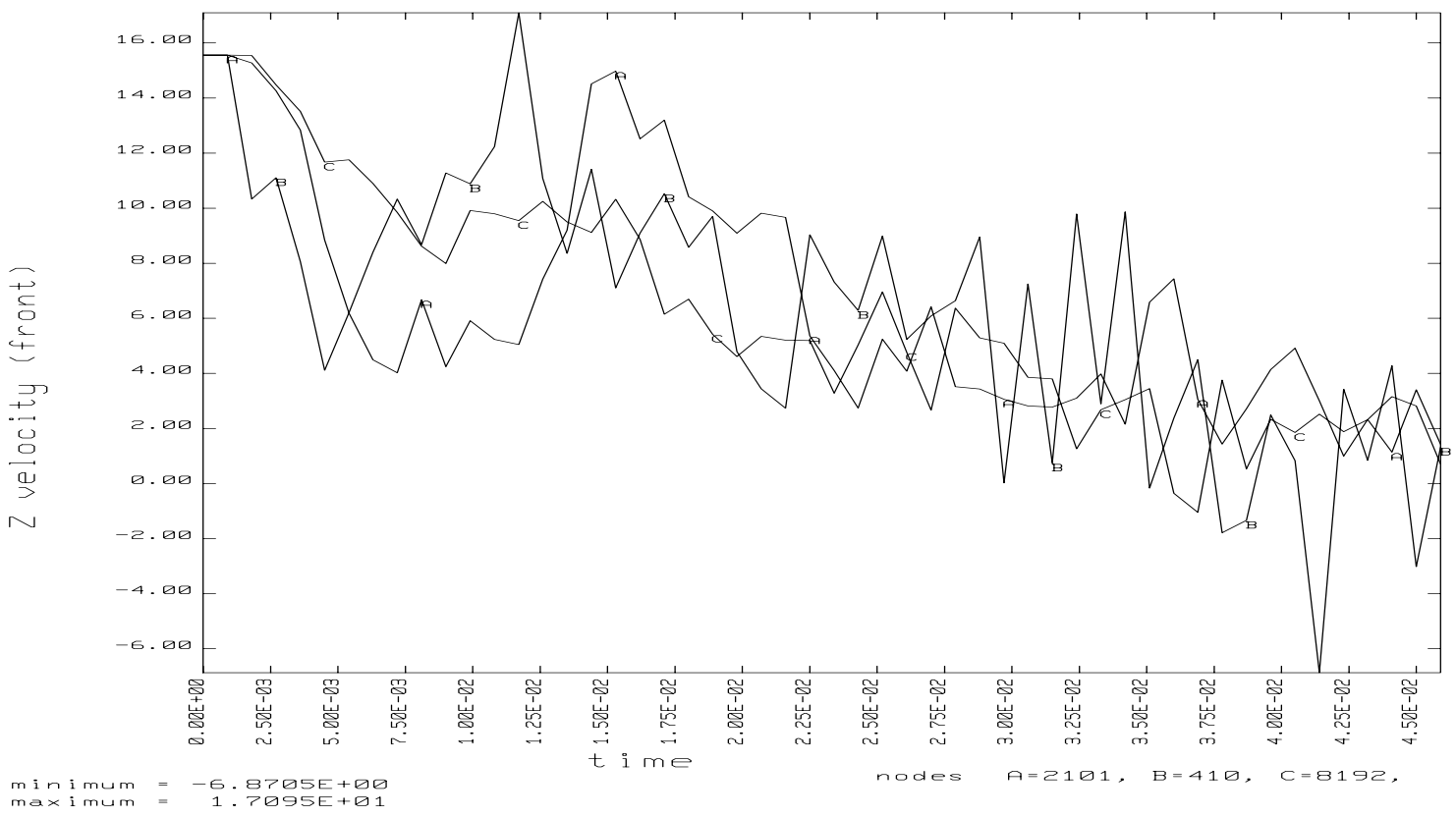

Fig 5.71 $\mathrm{Z}$ velocity for the nodes in the front of the vehicle for full frontal impact with Hughes-Liu SR thin shells.

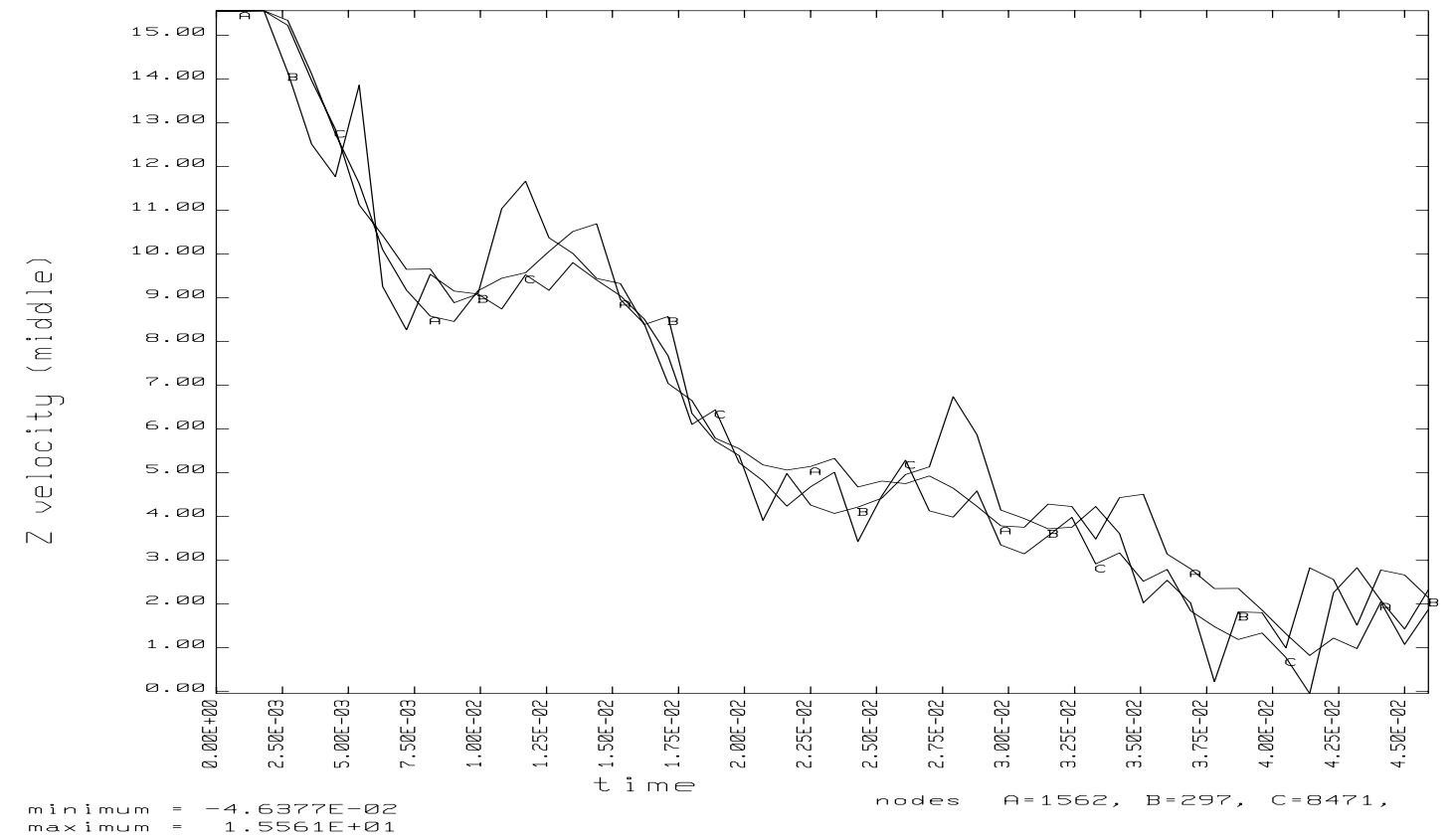

Fig 5.72 $\mathrm{Z}$ velocity for the nodes in the middle of the vehicle for full frontal impact with Hughes-Liu SR thin shells. 


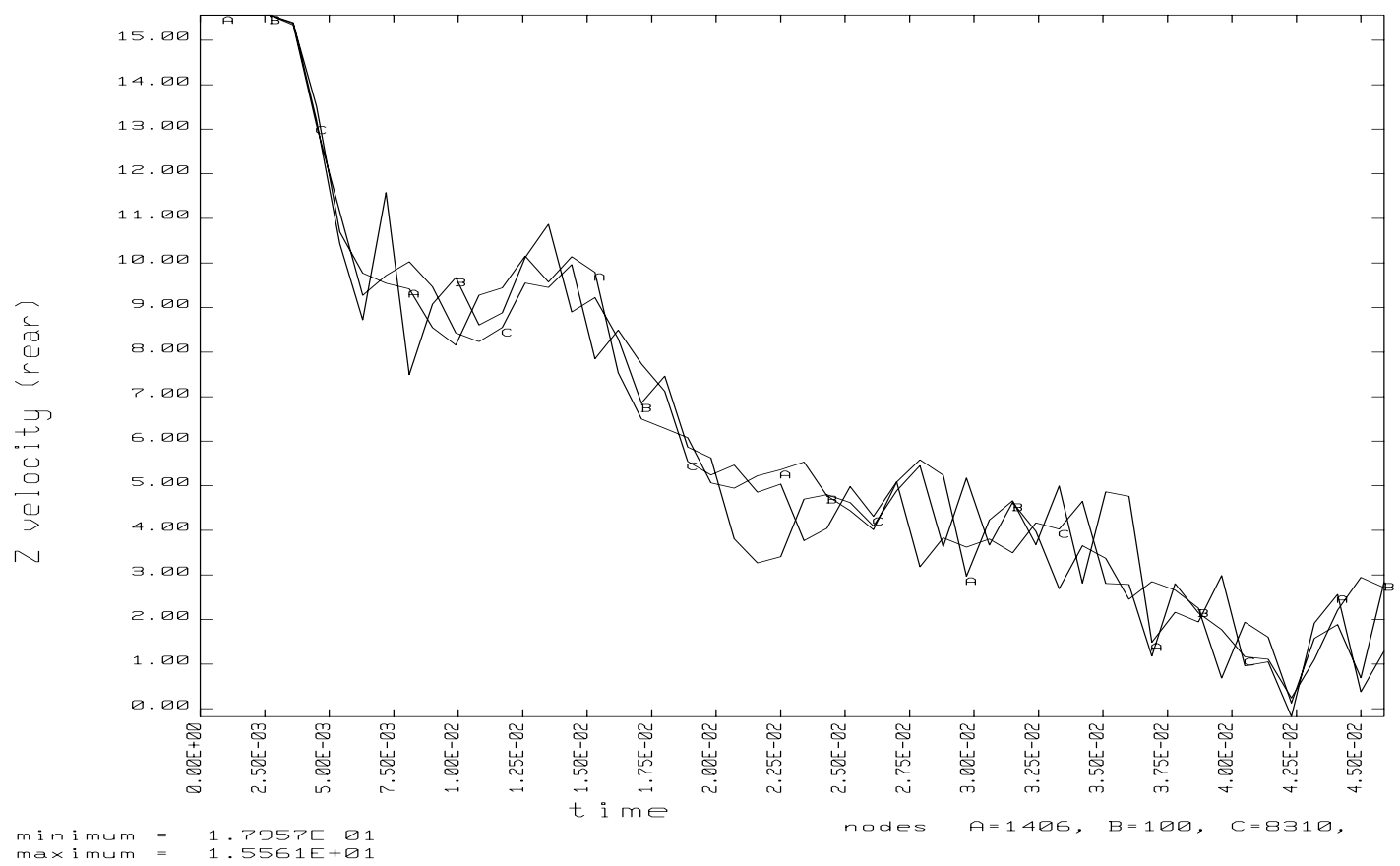

Fig 5.73 $\mathrm{Z}$ velocity for the nodes in the rear of the vehicle for full frontal impact with Hughes-Liu SR thin shells.

Figures 5.74, 5.75, and 5.76 show the $\mathrm{Z}$ acceleration of the nodes in the front, middle, and rear of the vehicle. The deceleration of the nodes in the front of the vehicle is higher than the deceleration of the nodes in the middle and the rear of the vehicle. The deceleration of the node in rear of the vehicle is higher than the deceleration of the node in the middle of the vehicle, which causes the bulging of the outer skin as seen in Figure 5.65. The deceleration of the nodes in the front of the vehicle in this case is higher than the deceleration of the nodes in the front of the vehicle without Hughes-Liu SR thin shells. The $\mathrm{Z}$ acceleration of the nodes in the front shows high oscillations about zero, whereas the oscillations of the nodes in the middle and the rear of the vehicle are less. 


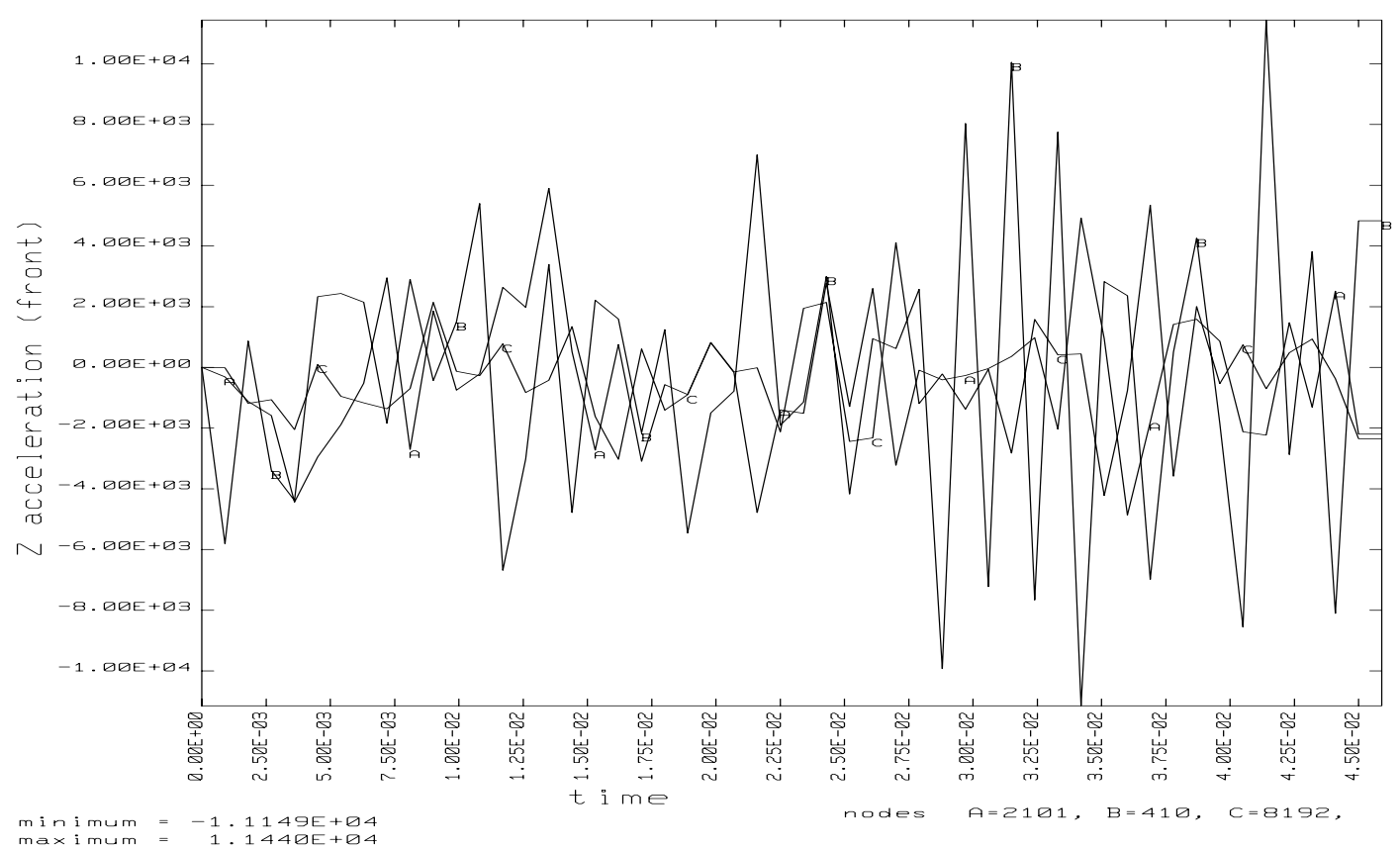

Fig 5.74 $\mathrm{Z}$ acceleration for the nodes in the front of the vehicle for full frontal impact with Hughes-Liu SR thin shells.

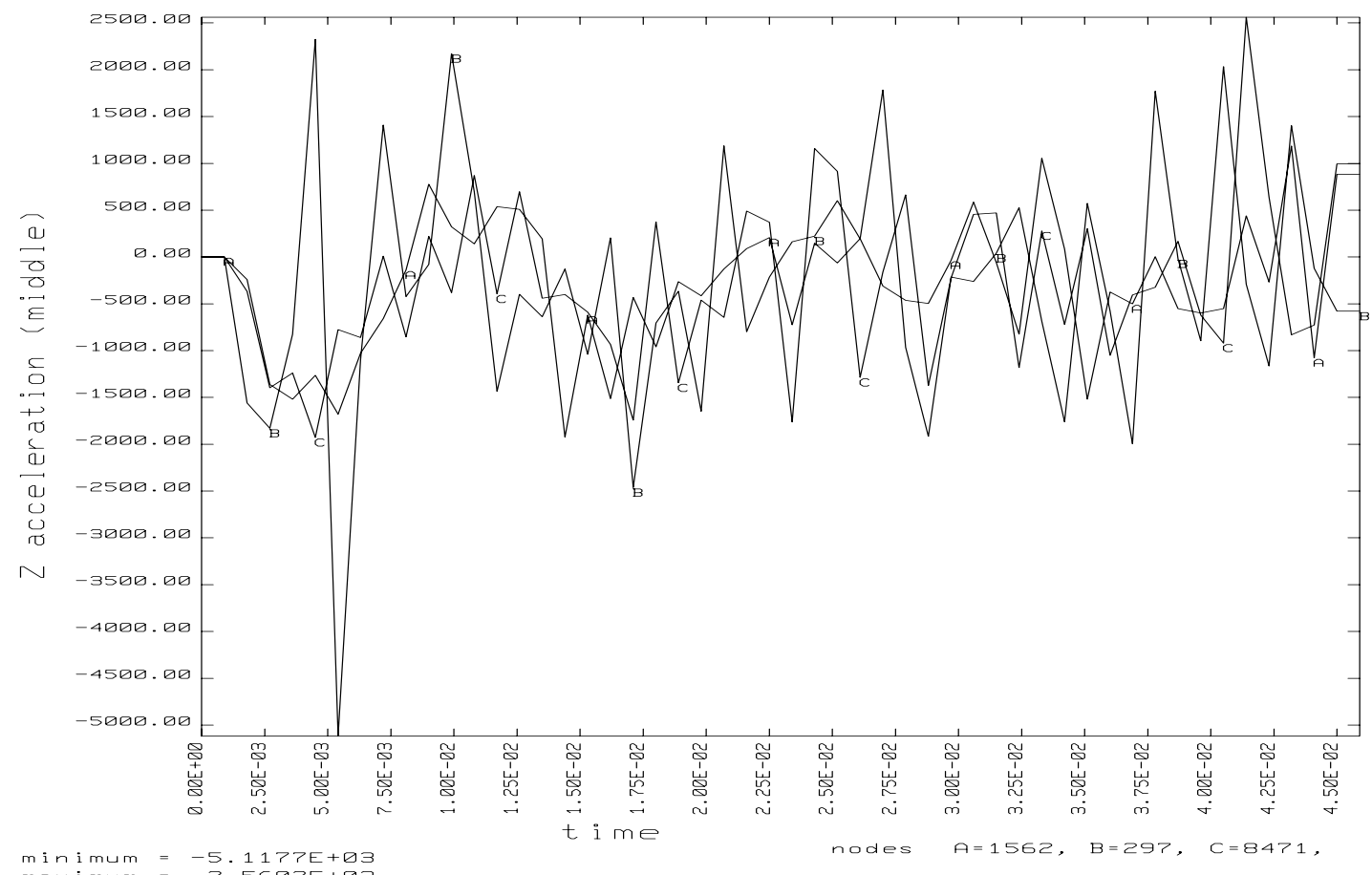

Fig 5.75 $\mathrm{Z}$ acceleration for the nodes in the middle of the vehicle for full frontal impact with Hughes-Liu SR thin shells. 


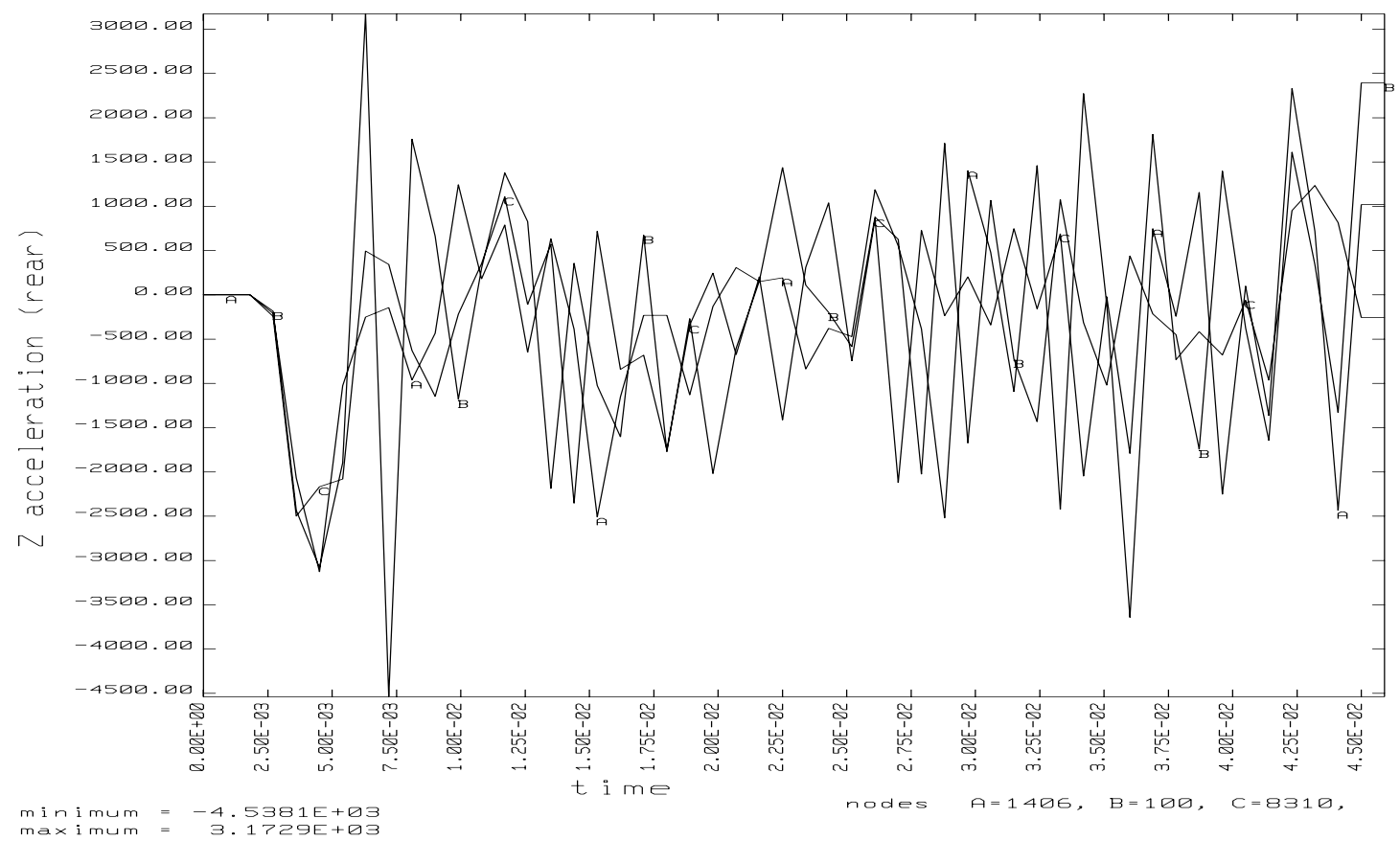

Fig 5.76 $\mathrm{Z}$ acceleration for the nodes in the rear of the vehicle for full frontal impact with Hughes-Liu SR thin shells.

Figures 5.77, 5.78, and 5.79 show the $\mathrm{X}, \mathrm{Y}$ and $\mathrm{Z}$ rigid body velocities respectively for the bus subjected to a full frontal impact with Hughes-Liu SR thin shells. From Figure 5.77 it can be seen that the $\mathrm{X}$ rigid body velocity of the materials in the $\mathrm{X}$ direction is less compared to the rigid body velocity of the materials in the $\mathrm{Y}$ and the $\mathrm{Z}$ direction, as seen in Figures 5.78 and 5.79. The hood has the highest $\mathrm{X}$ rigid body velocity and this is supported by the deformation of the hood in the $\mathrm{X}$ direction as seen in Figure 5.65. The $\mathrm{Y}$ rigid body velocity is high for the radiator and the engine since the frame pitches down as it hits the rigid wall. The $\mathrm{Z}$ rigid body velocity of the materials approaches a constant value as the time nears $50 \mathrm{msec}$, which shows that the decelerations are nearing zero and the problem is approaching a steady state. This phenomena is supported by the Figure 5.80, which shows the total material rigid body velocities. 


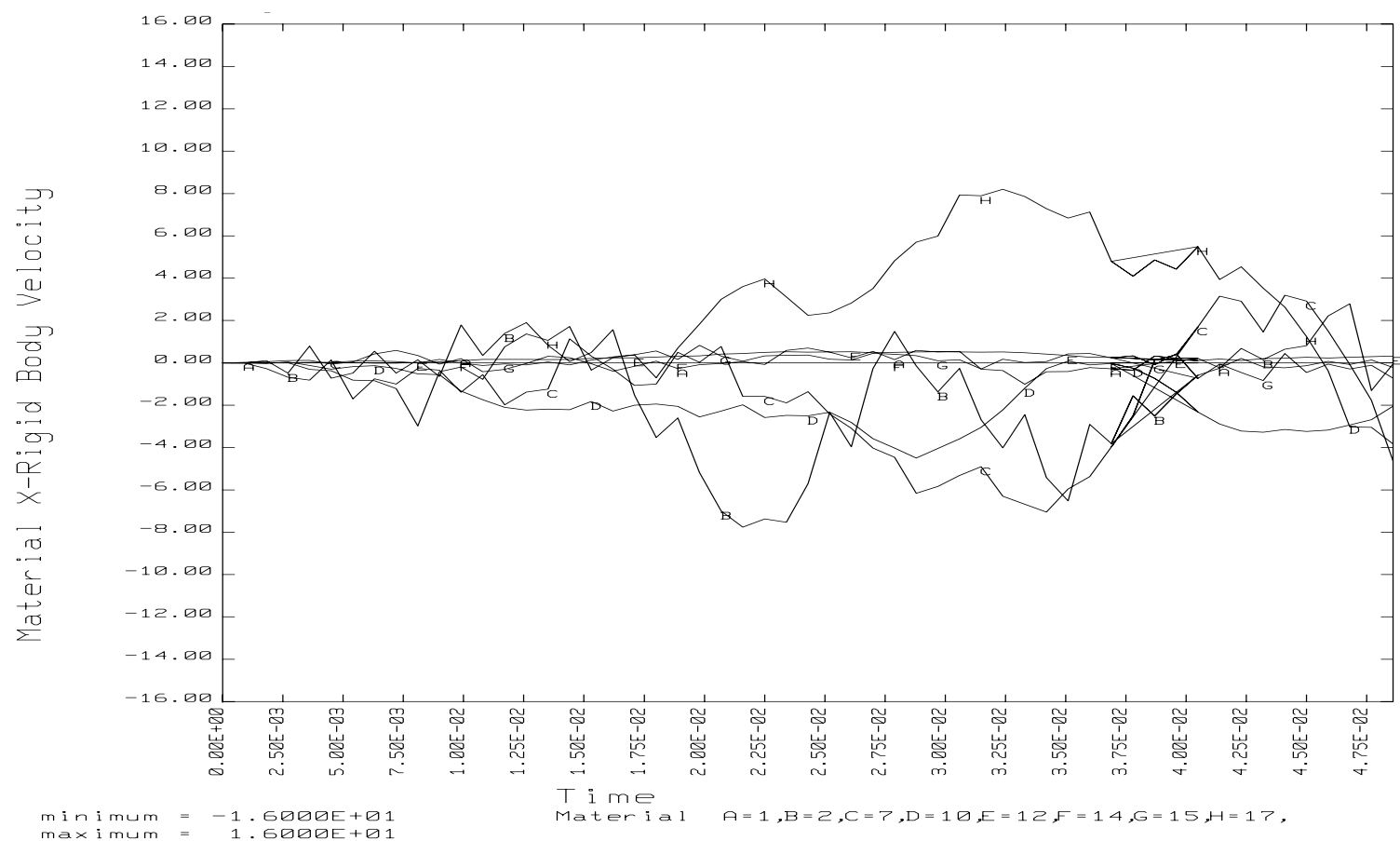

Fig 5.77 X rigid body velocities of materials for a full frontal impact with Hughes-Liu SR thin shells.

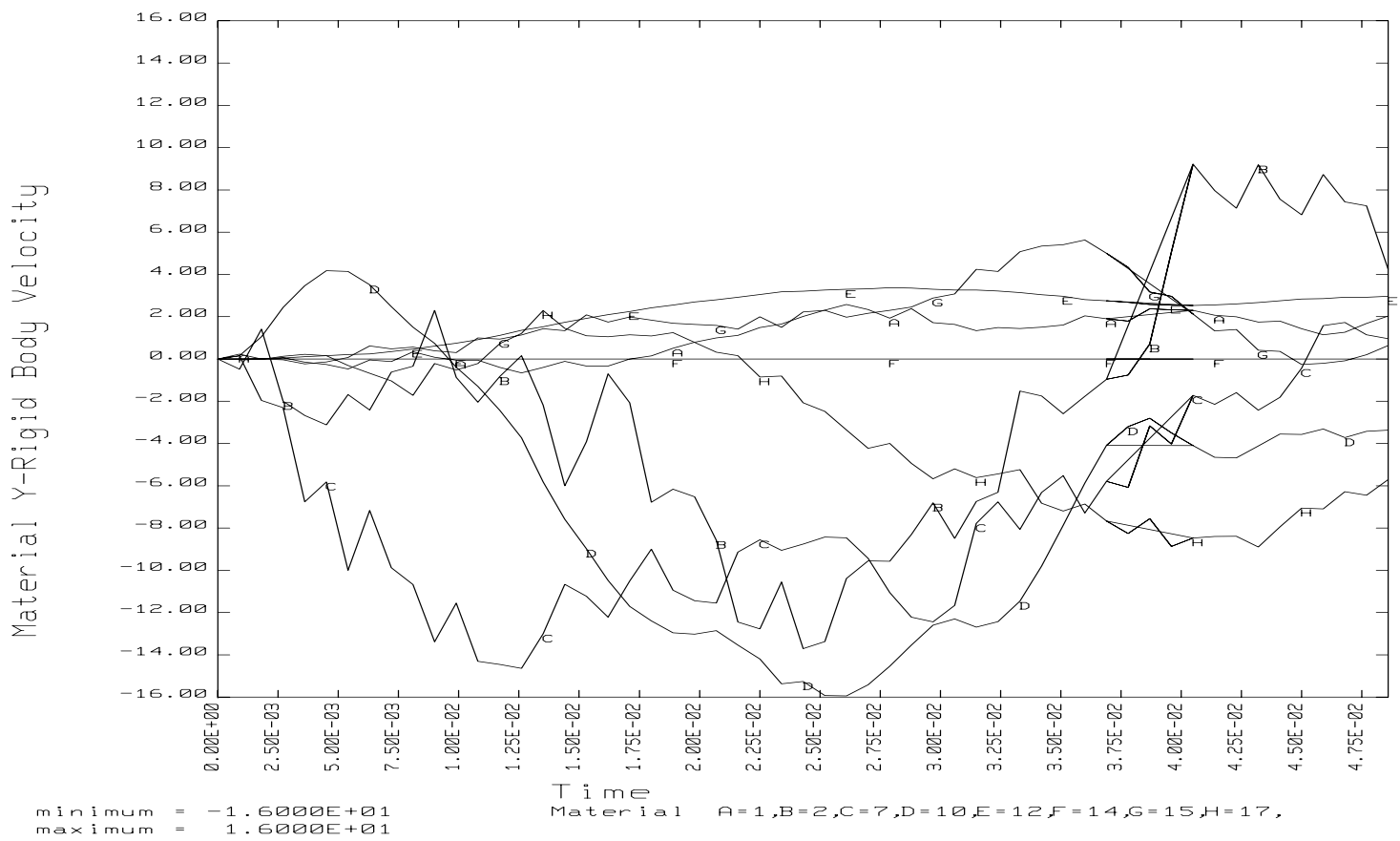

Fig 5.78 Y rigid body velocities of materials for a full frontal impact with Hughes-Liu SR thin shells. 


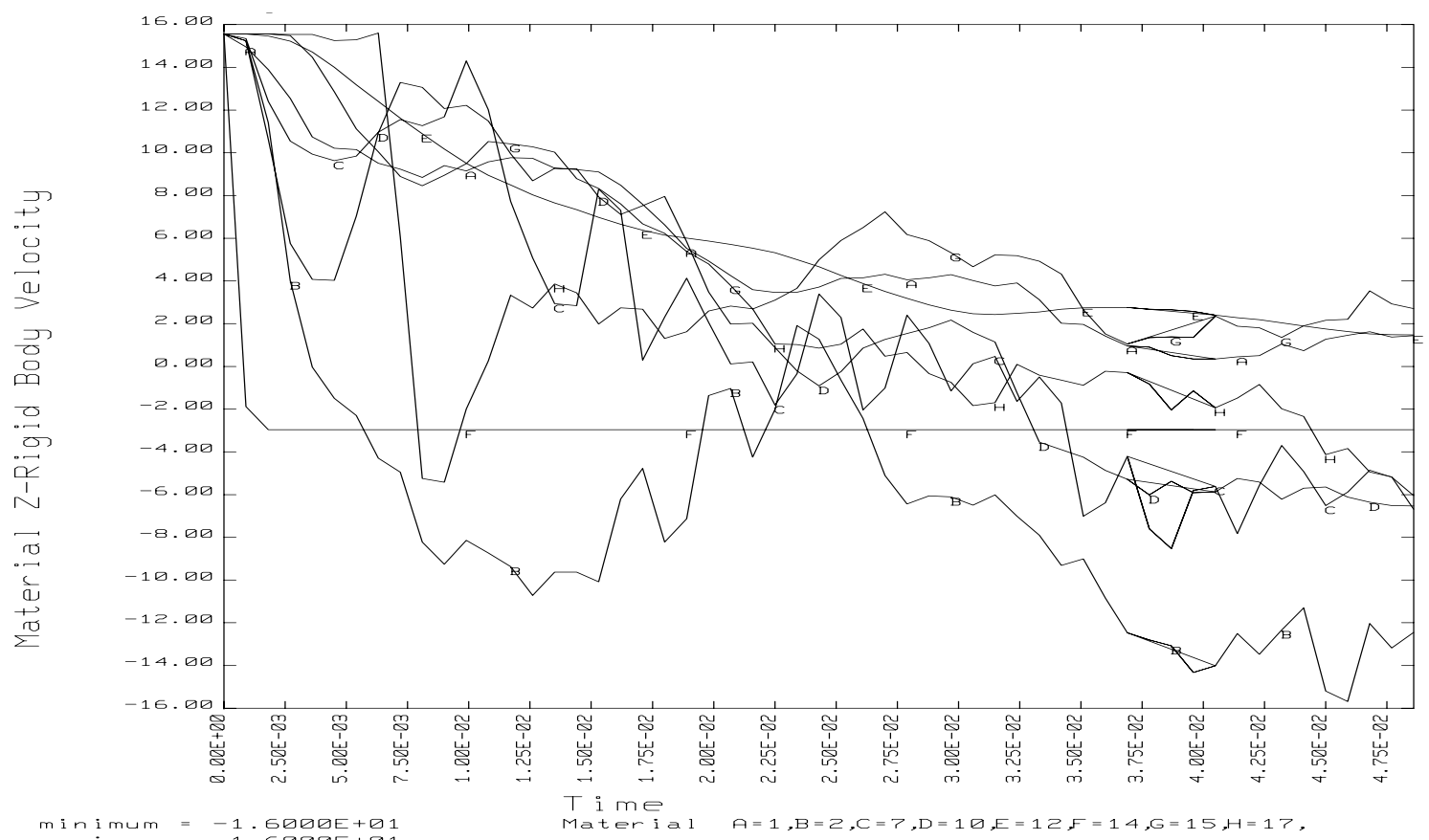

Fig 5.79 $\mathrm{Z}$ rigid body velocities of materials for a full frontal impact with Hughes-Liu SR thin shells.

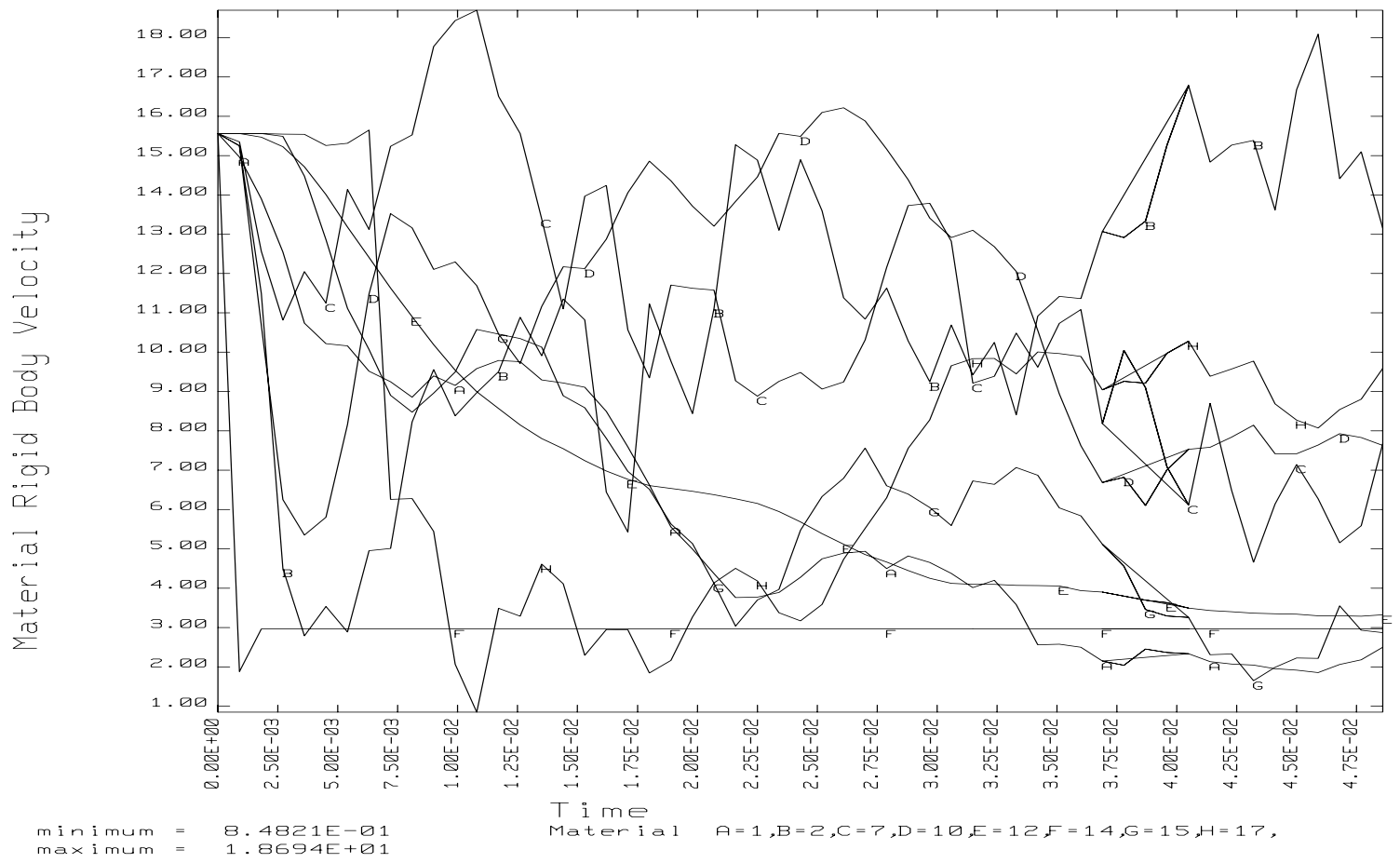

Fig 5.80 Total rigid body velocity for materials for a full frontal impact with Hughes-Liu SR thin shells. 
Figures 5.81, 5.82, and 5.83 show the global $\mathrm{X}, \mathrm{Y}$ and $\mathrm{Z}$ rigid body velocities for a full frontal impact with Hughes-Liu SR thin shells. Figure 5.81 shows that the global X rigid body velocity is almost zero for the analysis. Figure 5.82 shows the global Y rigid body velocity of the bus and its magnitude is to the order of one fifth of the $\mathrm{Z}$ rigid body velocity as seen in Figure 5.83. The total $\mathrm{Z}$ rigid body velocity almost reaches zero at the end of $47 \mathrm{msec}$ as seen from the graph in Figure 5.83. This shows that the vehicle has reached a steady state where there are no decelerations in the system anymore. Figure 5.84 shows the global total rigid body velocity for the bus and the velocity remains constant after $35 \mathrm{msec}$, which shows that the solution is approaching a steady state. The total rigid body velocity is the resultant of the $\mathrm{X}, \mathrm{Y}$ and $\mathrm{Z}$ rigid body velocities of the system.

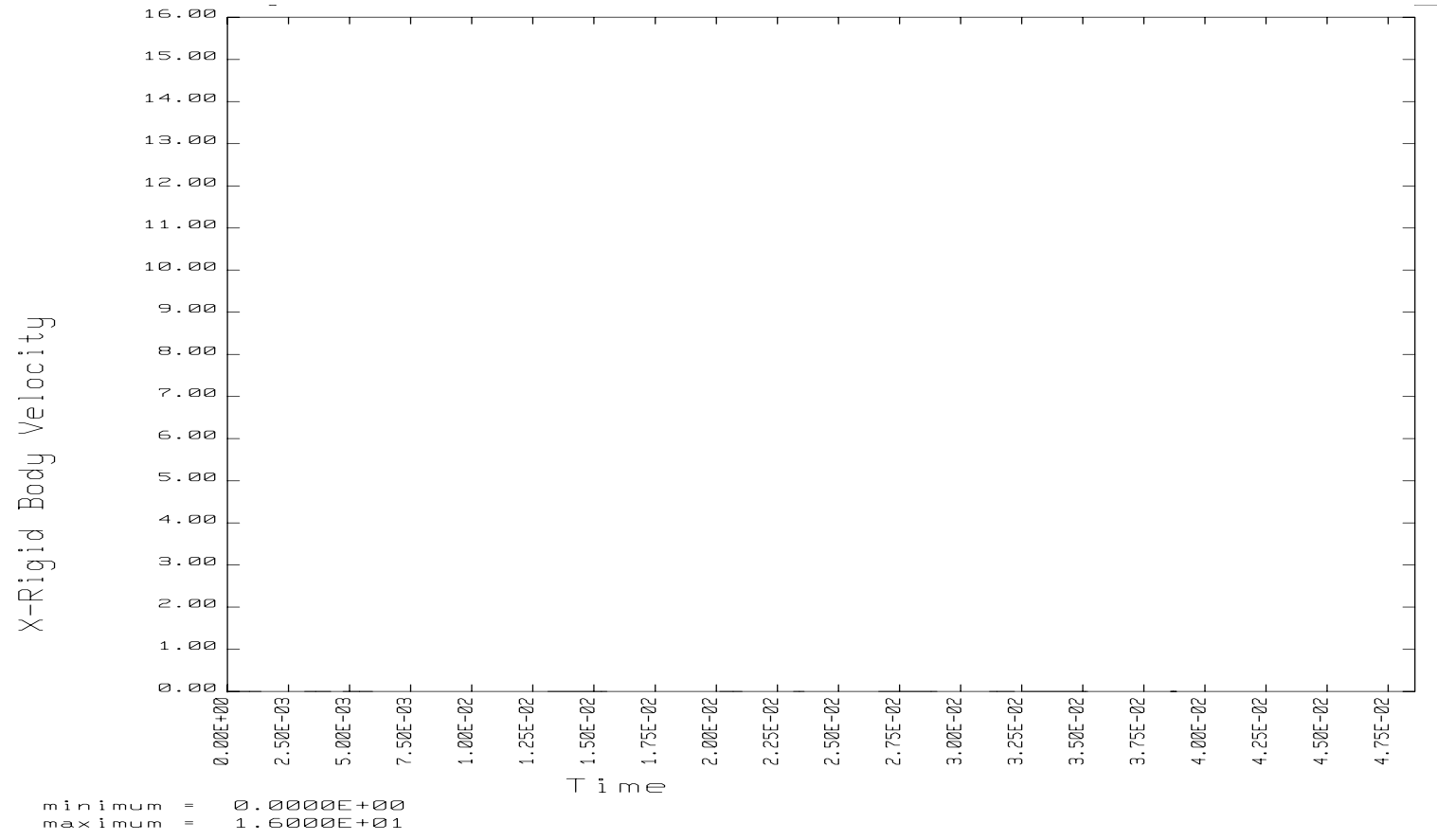

Fig 5.81 Global X rigid body velocity for a full frontal impact with Hughes-Liu SR thin shell. 


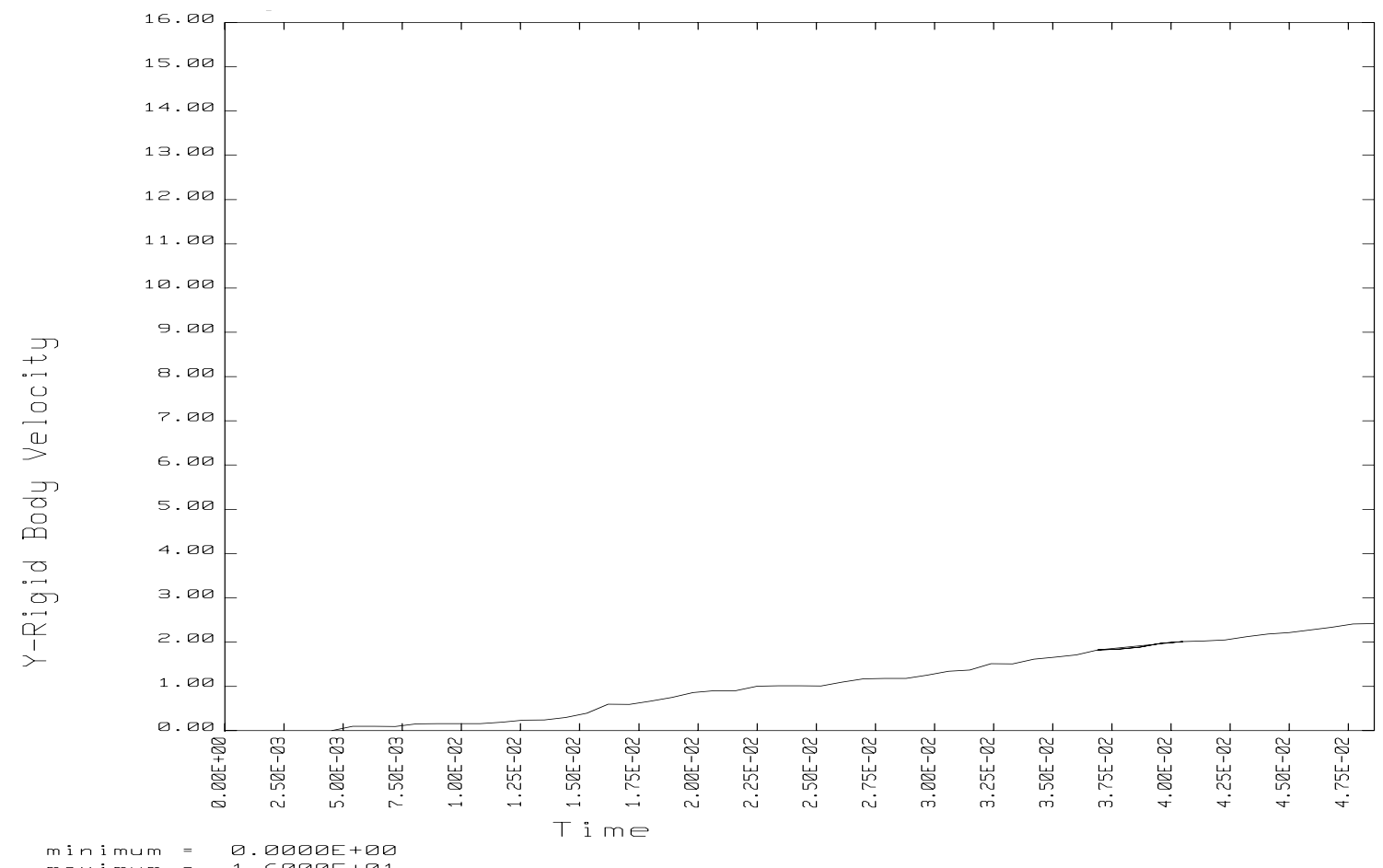

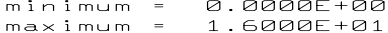

Fig 5.82 Global Y rigid body velocity for a full frontal impact with Hughes-Liu SR thin shell.

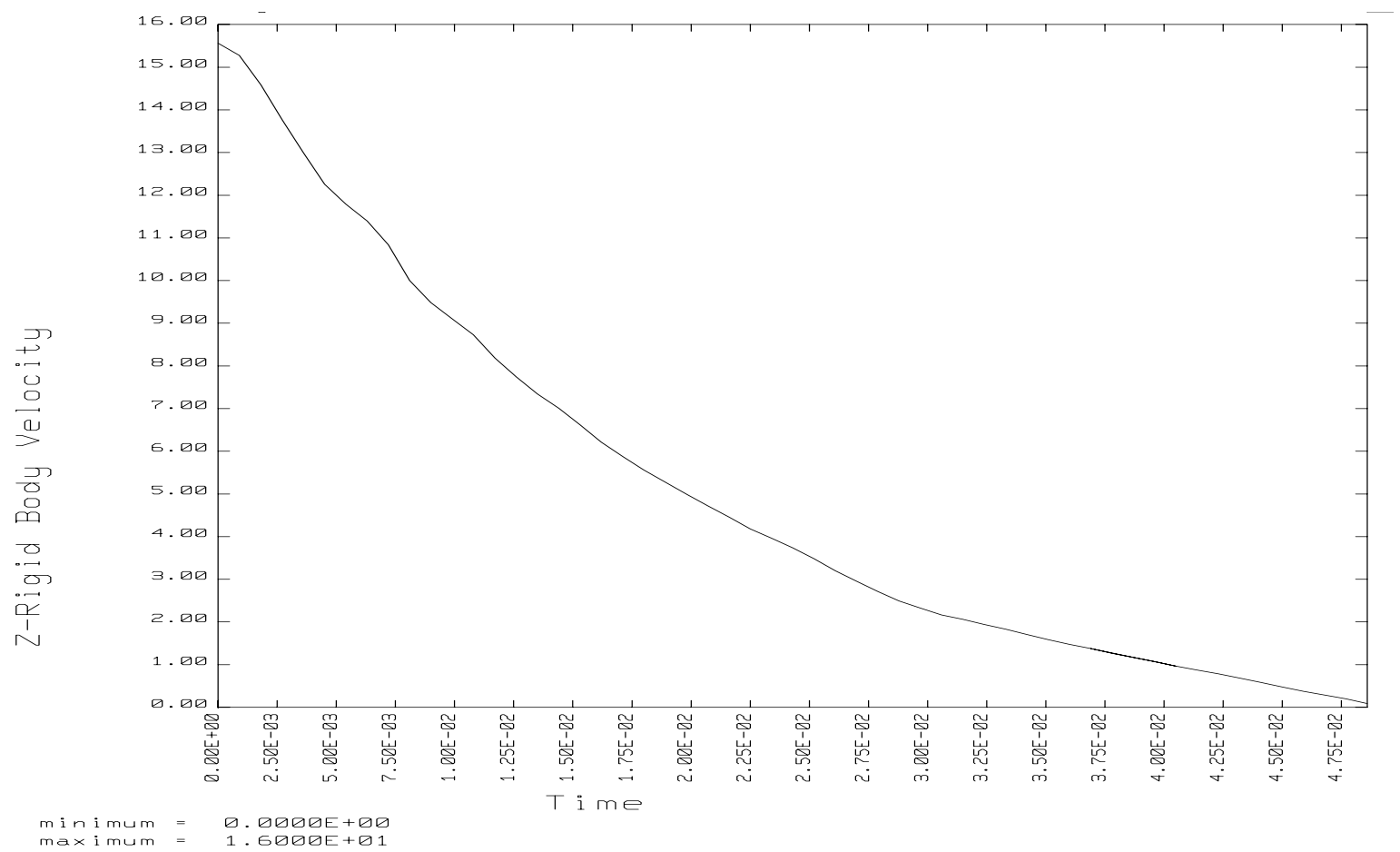

Fig 5.83 Global $Z$ rigid body velocity for a full frontal impact with Hughes-Liu SR thin shell. 


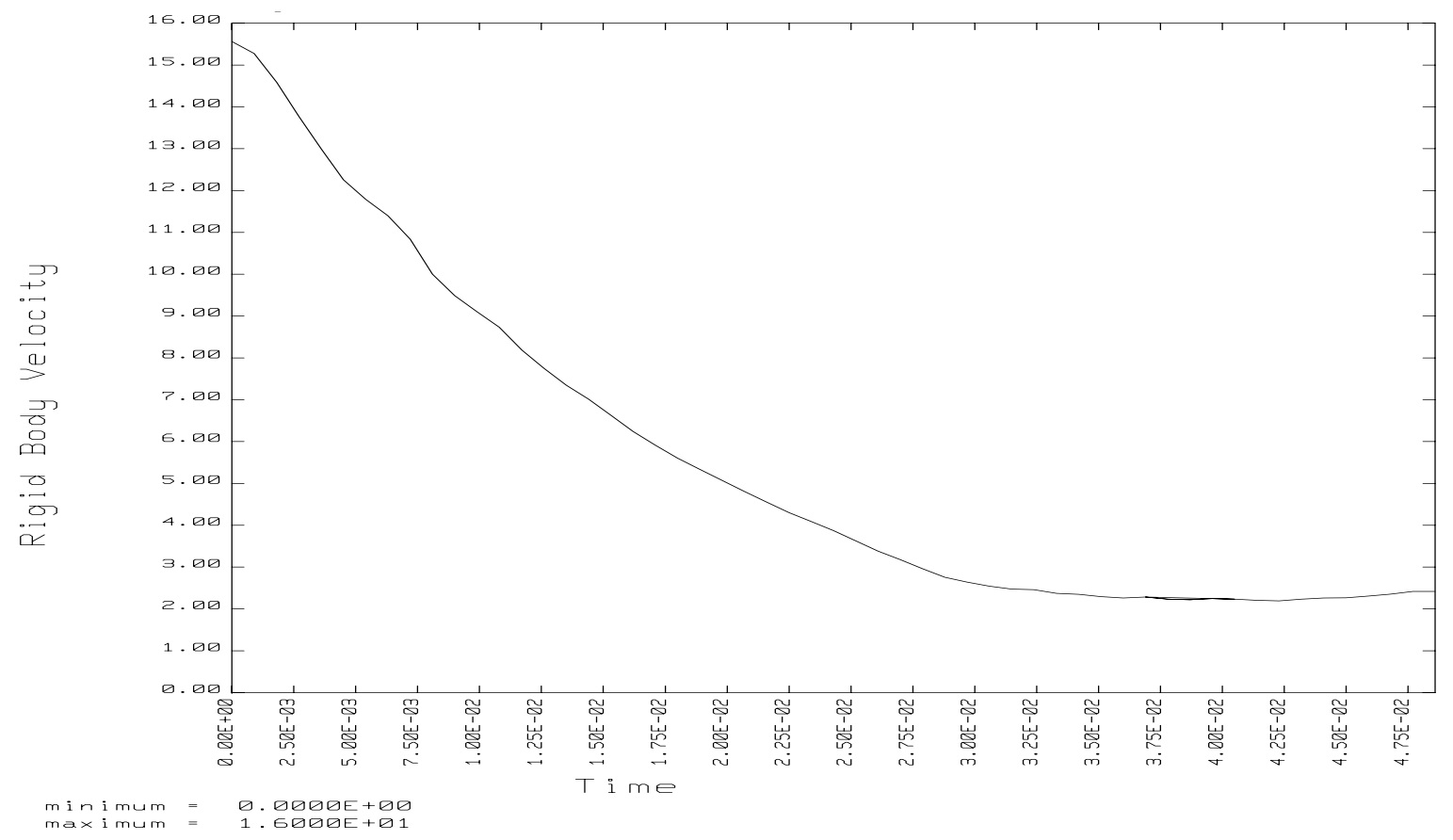

Fig 5.84 Global total rigid body velocity for a full frontal impact with Hughes-Liu SR thin shells.

Figures 5.85 shows the internal energy absorbed by different components of the bus. The frame absorbs the maximum energy as it buckles after the second engine mounting cross member. This can be seen from the graph in Figure 5.85. This behavior is the same for the full frontal impact without Hughes-Liu SR thin shells, but the magnitude of the energy absorbed in this case is higher because of the presence of hourglassing energy in the previous system. This can be seen from comparing the graphs in Figure 5.28, and 5.85. Figure 5.86 shows the global energies for a full frontal impact with Hughes-Liu SR thin shells. Unlike the case of a full frontal impact without Hughes-Liu SR thin shells, the sum of the internal and the kinetic energies is equal to the total energy. It can be seen that the other energies in the system are zero or negligible.

The pie chart in Figure 5.87 at the end of the analysis shows the internal energy distribution at time $=45 \mathrm{msec}$ for the bus subjected to a full frontal impact with Hughes- 
Liu SR thin shells. Compared to the pie chart in Figure 5.30 at the end of the analysis, which shows the internal energy distribution for a full frontal impact without Hughes-Liu SR thin shells, the percentage of energy absorbed in this case is lesser although the magnitude of the energy absorbed is higher. However, in both the cases, the frame absorbs the maximum energy at the end of the analysis as illustrated in Figures 5.30 and 5.87.

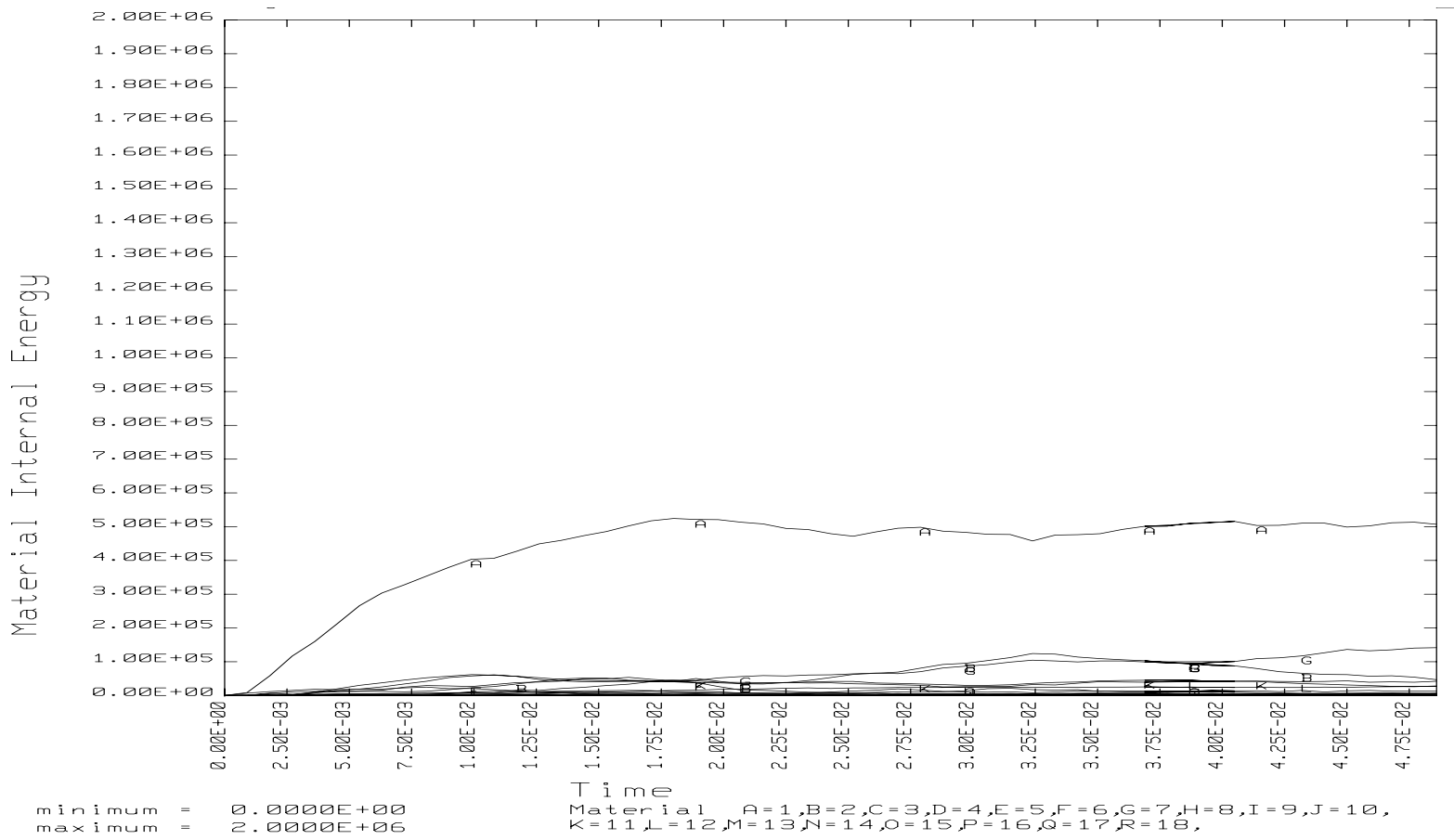

Fig 5.85 Material internal energy absorption for a full frontal impact with Hughes-Liu SR thin shells. 


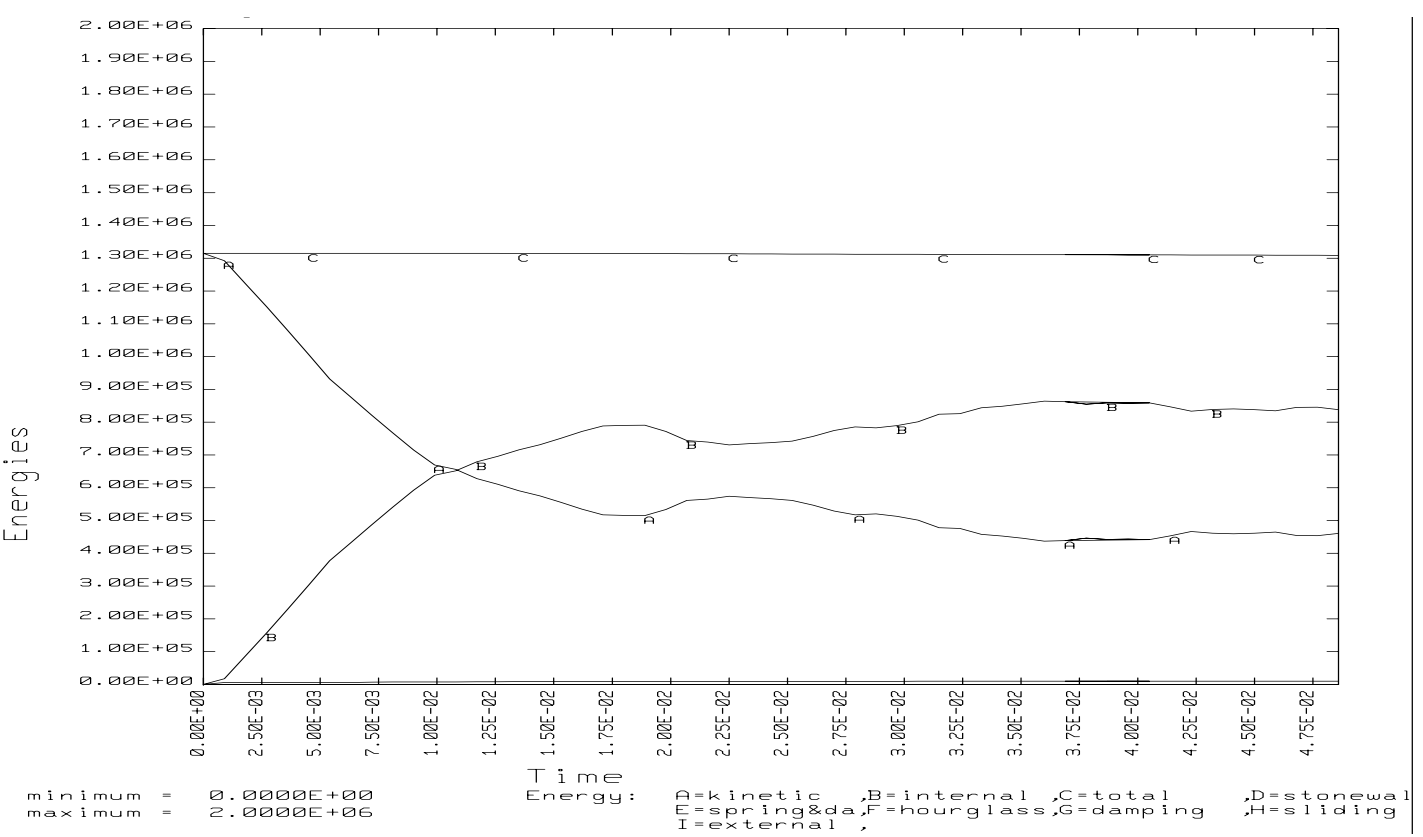

Fig 5.86 Global energies for a full frontal impact with Hughes-Liu SR thin shells.

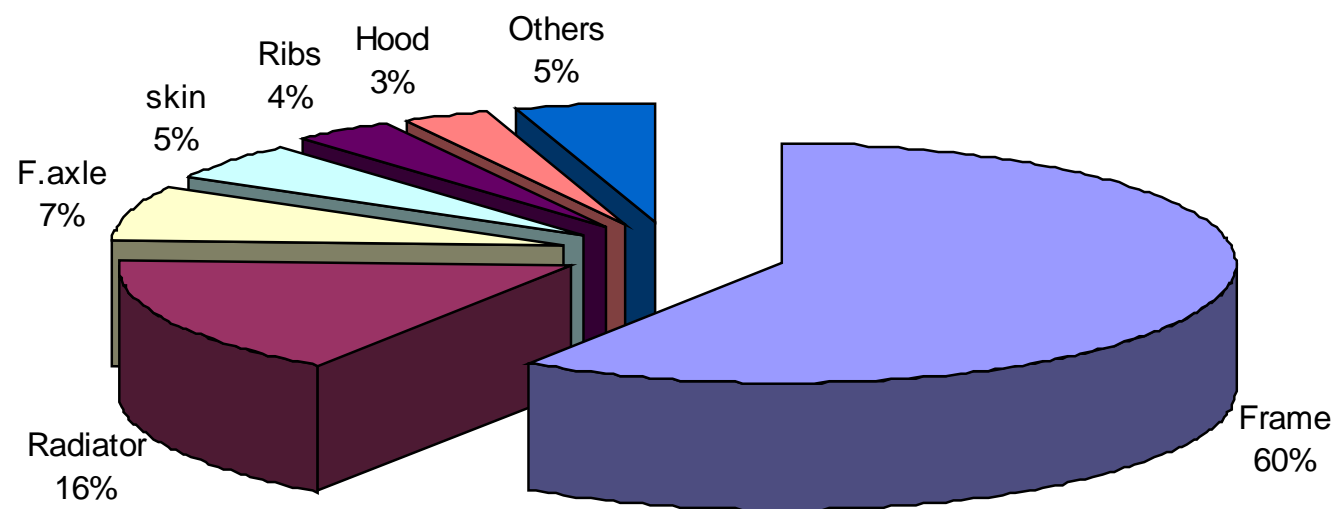

Fig 5.87 Total internal energy distribution at time $=\mathbf{4 5}$ msec of the bus subjected to a full frontal impact with Hughes-Liu SR thin shells. 


\subsection{OfFSET Frontal IMPaCt With Hughes-LiU SR Thin SHELl Elements}

Figure 5.88 shows the front view at time $=0 \mathrm{msec}$ of the bus subjected to a full frontal impact with Hughes-Liu SR thin shell elements. The initial conditions and the boundary conditions were the same for both analyses run with the single point integration and the Hughes-Liu SR thin shell elements. The only difference between the two analyses is the element formulation for the thin shell elements.

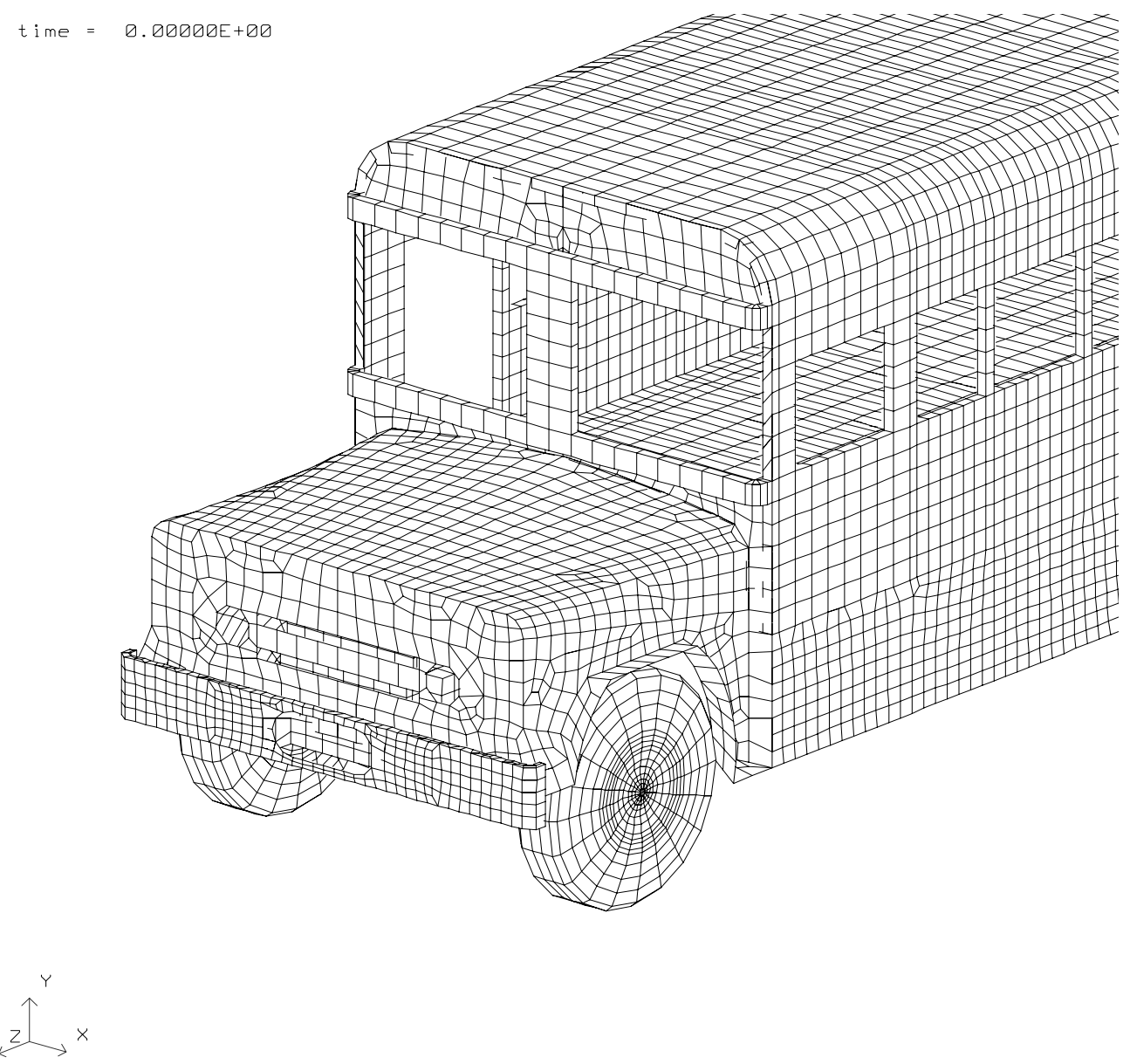

Fig 5.88 Front view at time $=0$ msec of the bus subjected to an offset frontal impact with Hughes-Liu SR thin shells.

Figure 5.89 shows the front view at time $=9 \mathrm{msec}$ of the bus subjected to an offset frontal impact with Hughes-Liu SR thin shells. The deformation of the hood is only on 
one side of the hood as illustrated. The deformation at this time state is the same for the both cases with and without Hughes-Liu SR thin shells because of the absence of hourglassing energy in the initial stages of the analysis. This can be seen by comparing Figures 5.88 and 5.31. Since the principal direction of the impact force is offset from the line of symmetry of the hood, the front axle rotates and the right wheel (door side) moves in the negative $\mathrm{Z}$ direction. This can be seen from the graph in Figure 5.97.

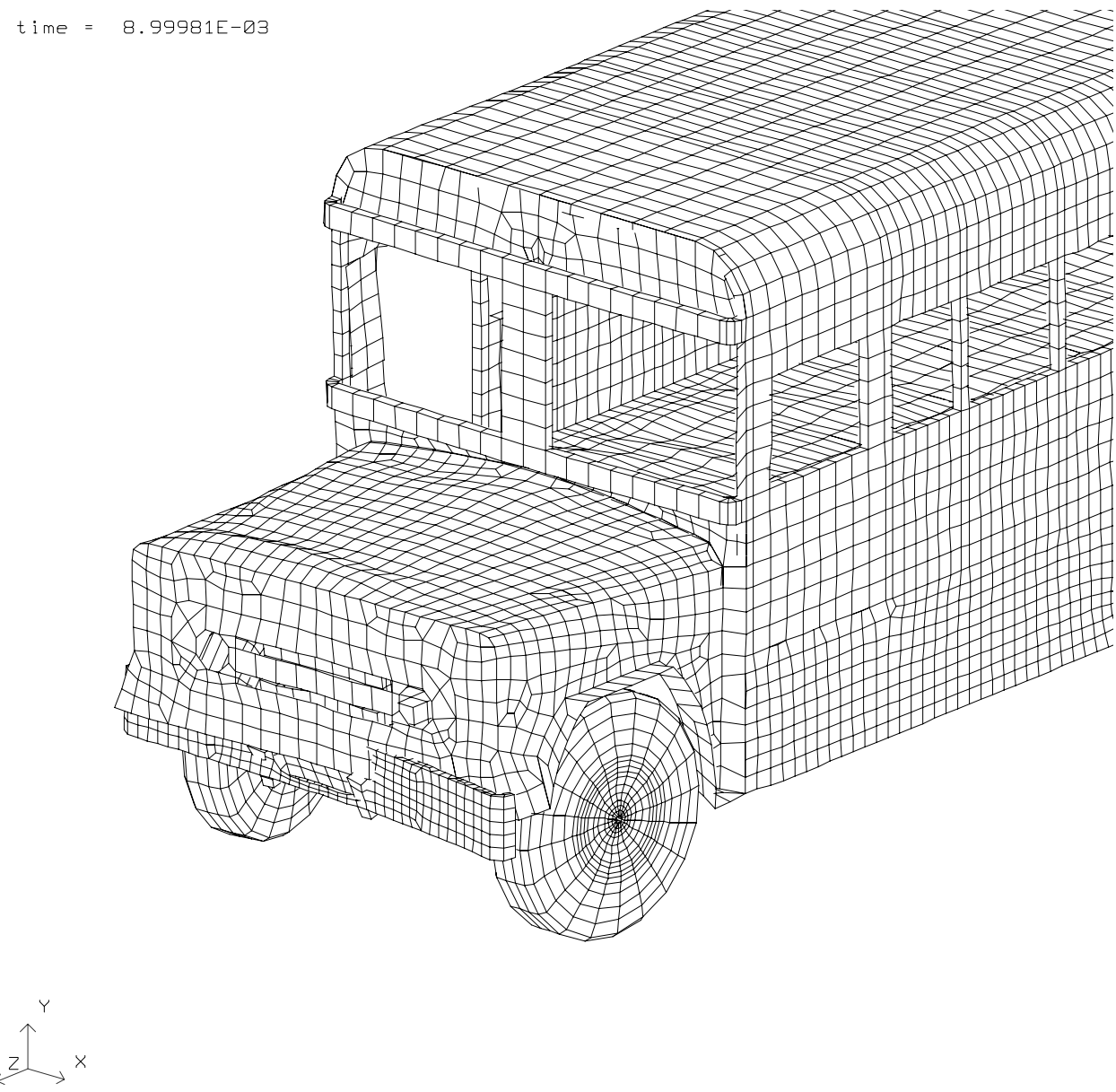

Fig 5.89 Front view at time $=9$ msec of the bus subjected to an offset frontal impact with Hughes-Liu SR thin shells.

Figure 5.90 shows the front view at time $=18 \mathrm{msec}$ of the bus subjected to an offset frontal impact with Hughes-Liu SR thin shell elements. The difference in Z displacement 
between the nodes in the right and the left tire increases, which shows the rotation of the front axle and in this case, the vehicle behaves in the same way as the offset frontal collision without Hughes-Liu SR thin shell elements. The velocity of the vehicle lessens and hence the kinetic energy of the system is reduced. The internal energy absorbed by the parts in the front end of the vehicle increases as the kinetic energy decreases and this can be seen in Figure 5.114.

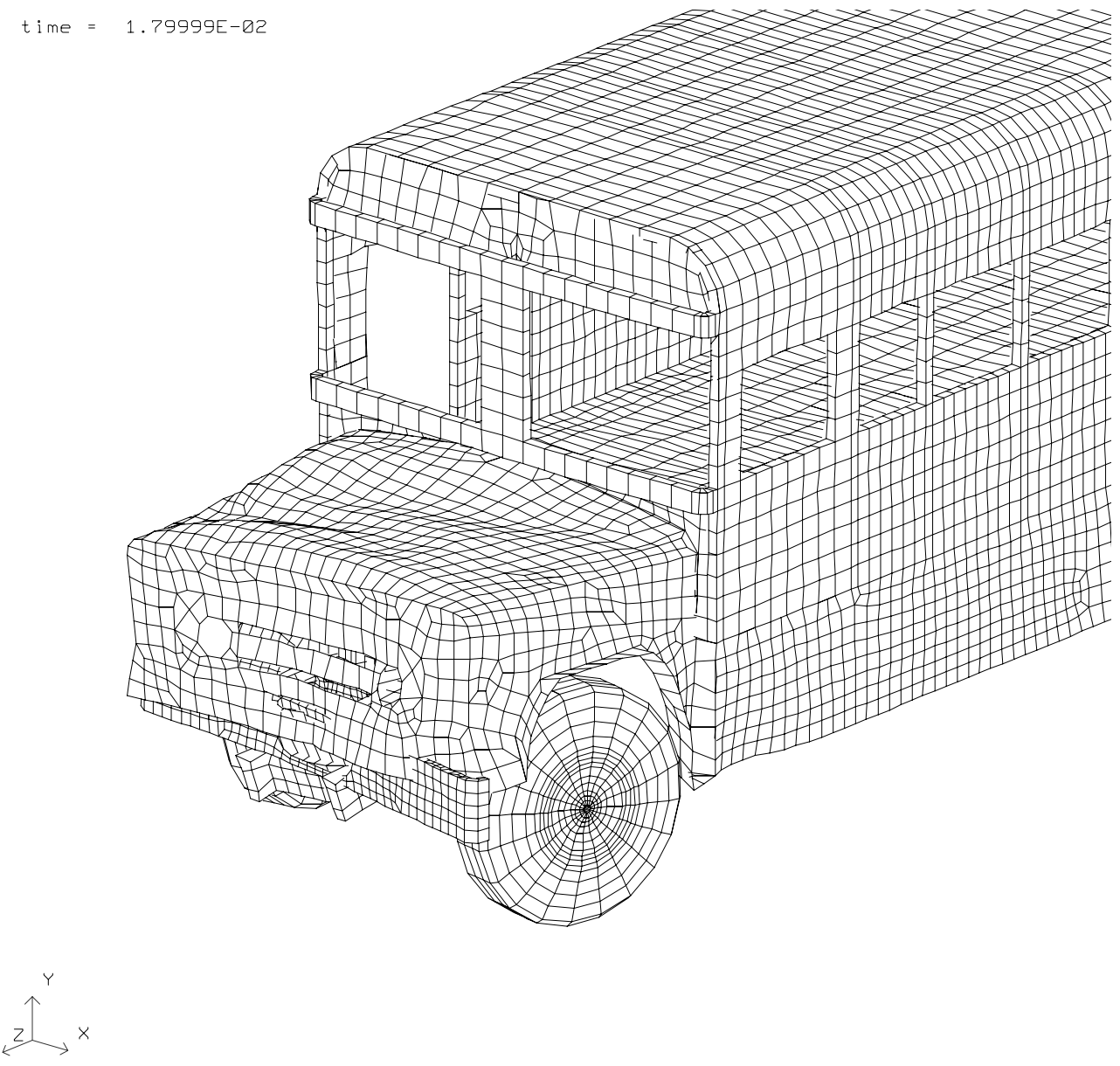

Fig 5.90 Front view at time $=18$ msec of the bus subjected to an offset frontal impact with Hughes-Liu SR thin shells.

Figure 5.91 shows the front view at time $=27 \mathrm{msec}$ of the bus subjected to an offset frontal impact with Hughes-Liu SR thin shell elements. The front tires move in the 
negative $\mathrm{Y}$ axis compared to the rear tire, and the vehicle pitches about the $\mathrm{Y}$ axis as seen in Figure 5.98. The magnitude of the pitching is higher in this case compared to the offset frontal impact without Hughes-Liu SR thin shells. The $\mathrm{Z}$ relative displacement between the right and the left tires on the front side is also high in this case compared to the offset frontal impact without Hughes-Liu SR thin shells, which means that the axle rotates more in this case. The kinetic and the internal energy curves meet at this time state and the rate of energy conversion is higher in this case compared to the case without Hughes-Liu SR thin shell elements. This can be seen by comparing the graphs in Figure 5.55 and Figure 5.114 .

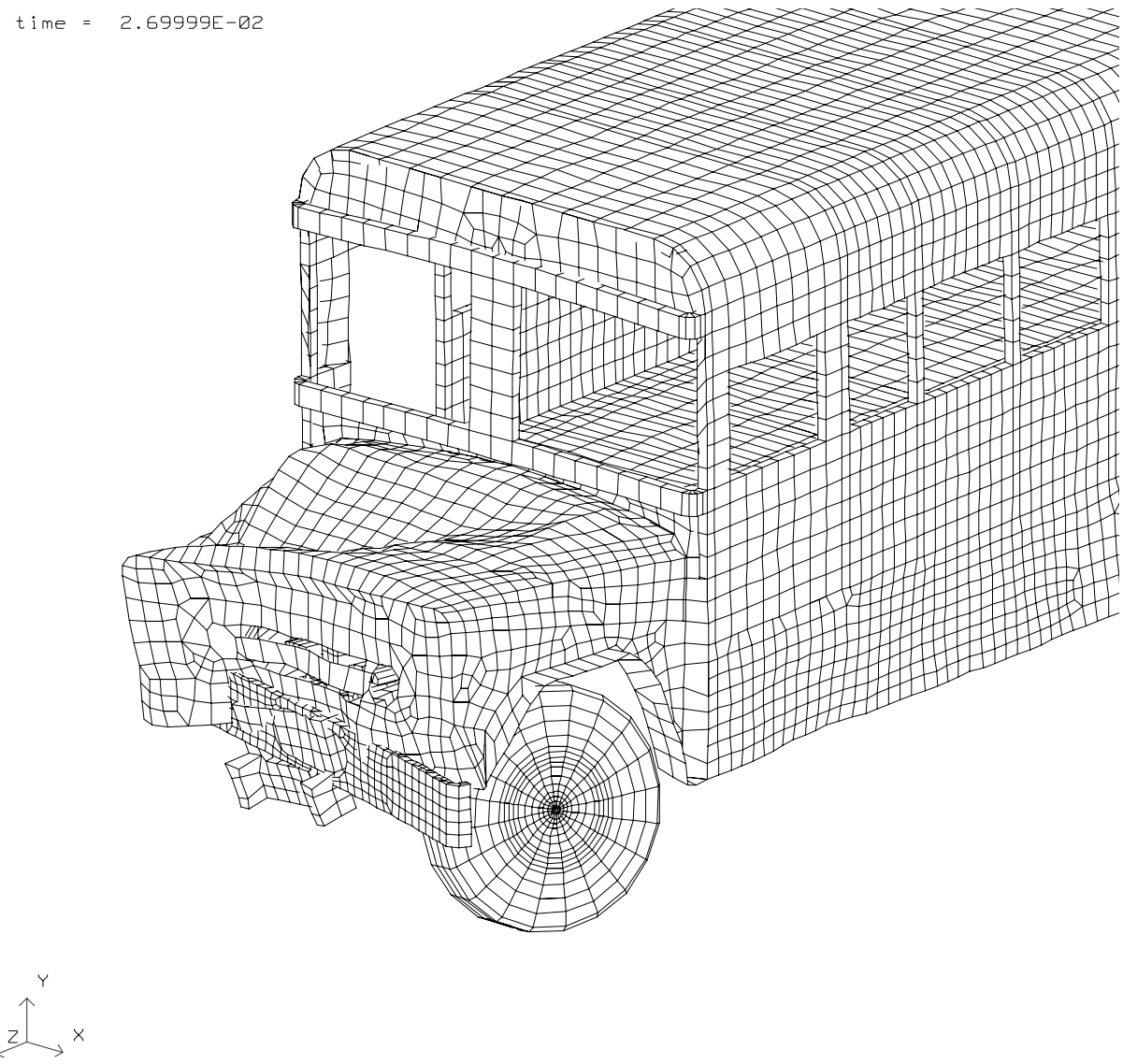

Fig 5.91 Front view at time $=27 \mathrm{msec}$ of the bus subjected to an offset frontal impact with Hughes-Liu SR thin shells. 
Figure 5.92 shows the front view at time $=36 \mathrm{msec}$ of the bus subjected to an offset frontal impact with Hughes-Liu SR thin shell elements. The frame bends after the second engine mounting cross member and it behaves similar to the case without Hughes-Liu SR thin shells. This may be because of the large distance between the second engine mounting cross member and the next cross member along the length of the frame. The maximum stress in the fire wall in this case is $241.1 \mathrm{MN} / \mathrm{m}^{2}$ as compared to $164 \mathrm{MN} / \mathrm{m}^{2}$ for an offset frontal impact without Hughes-Liu SR thin shell elements. This shows that the material of the fire wall has reached the plastic limit and any deformation here after will be plastic. The maximum absolute displacement of the fire wall is $0.39 \mathrm{~m}$ in the positive $\mathrm{Z}$ direction, which shows that even though there is a plastic deformation, there is no intrusion into the occupant area. 


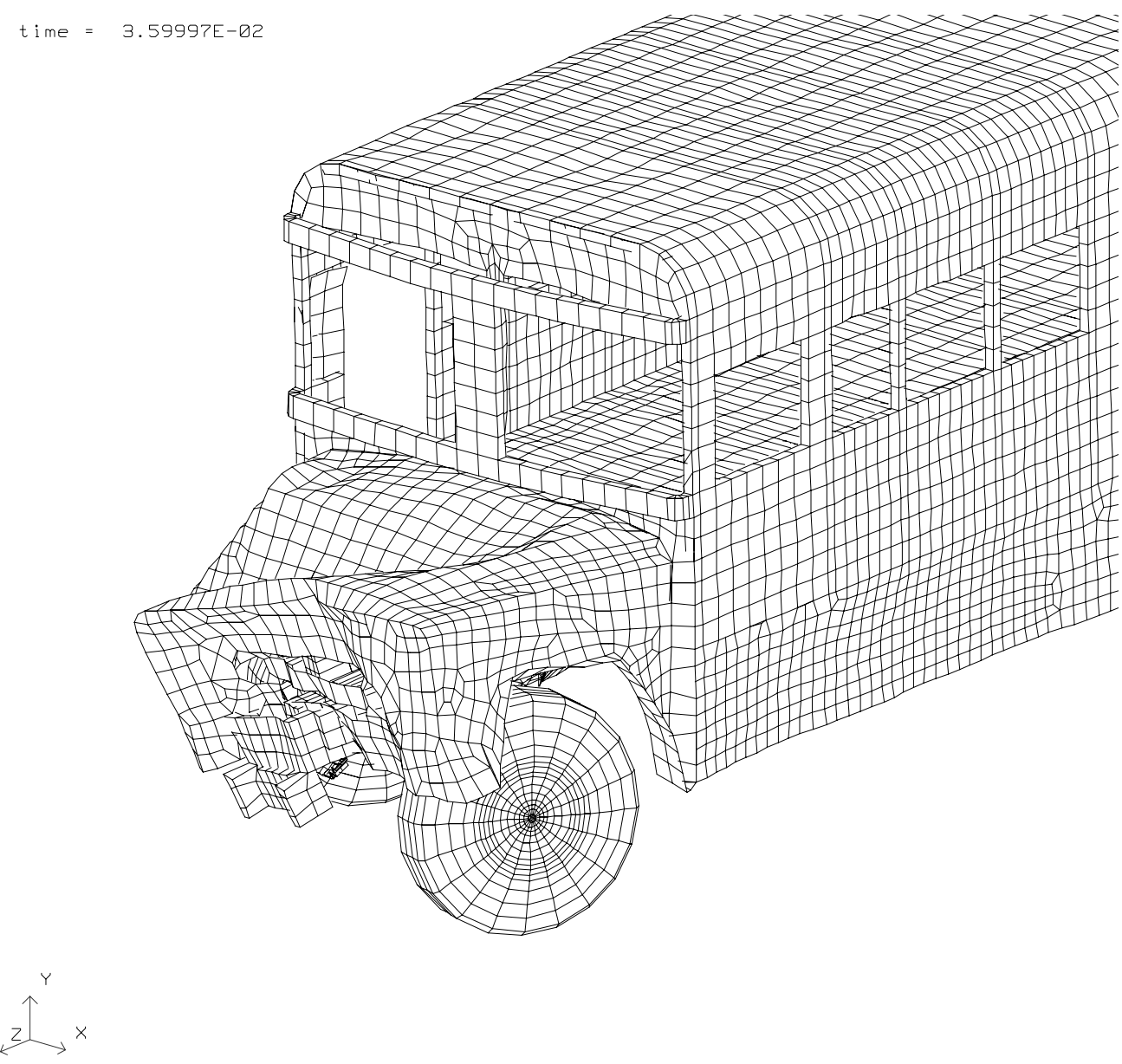

Fig 5.92 Front view at time $=\mathbf{3 6}$ msec of the bus subjected to an offset frontal impact with Hughes-Liu SR thin shells.

Figure 5.93 shows the front view at time $=45 \mathrm{msec}$ of the bus subjected to an offset frontal impact with Hughes-Liu SR thin shells. The front grill structure in the hood has failed and the frame protrudes through the hood as illustrated. The dent formed in the hood on the impact side in this case is similar to the offset frontal impact without Hughes-Liu SR thin shells, and this can be seen by comparing Figures 5.35 and 5.93. The internal energy absorbed by the parts increases and the kinetic energy decreases as seen from the graph in Figure 5.114. The maximum internal energy is absorbed by the frame in this case, which is similar to the case without Hughes-Liu SR thin shell elements. 
However the magnitude of the internal energy absorbed is higher in this case and this can be seen by comparing Figures 5.54 and 5.113.

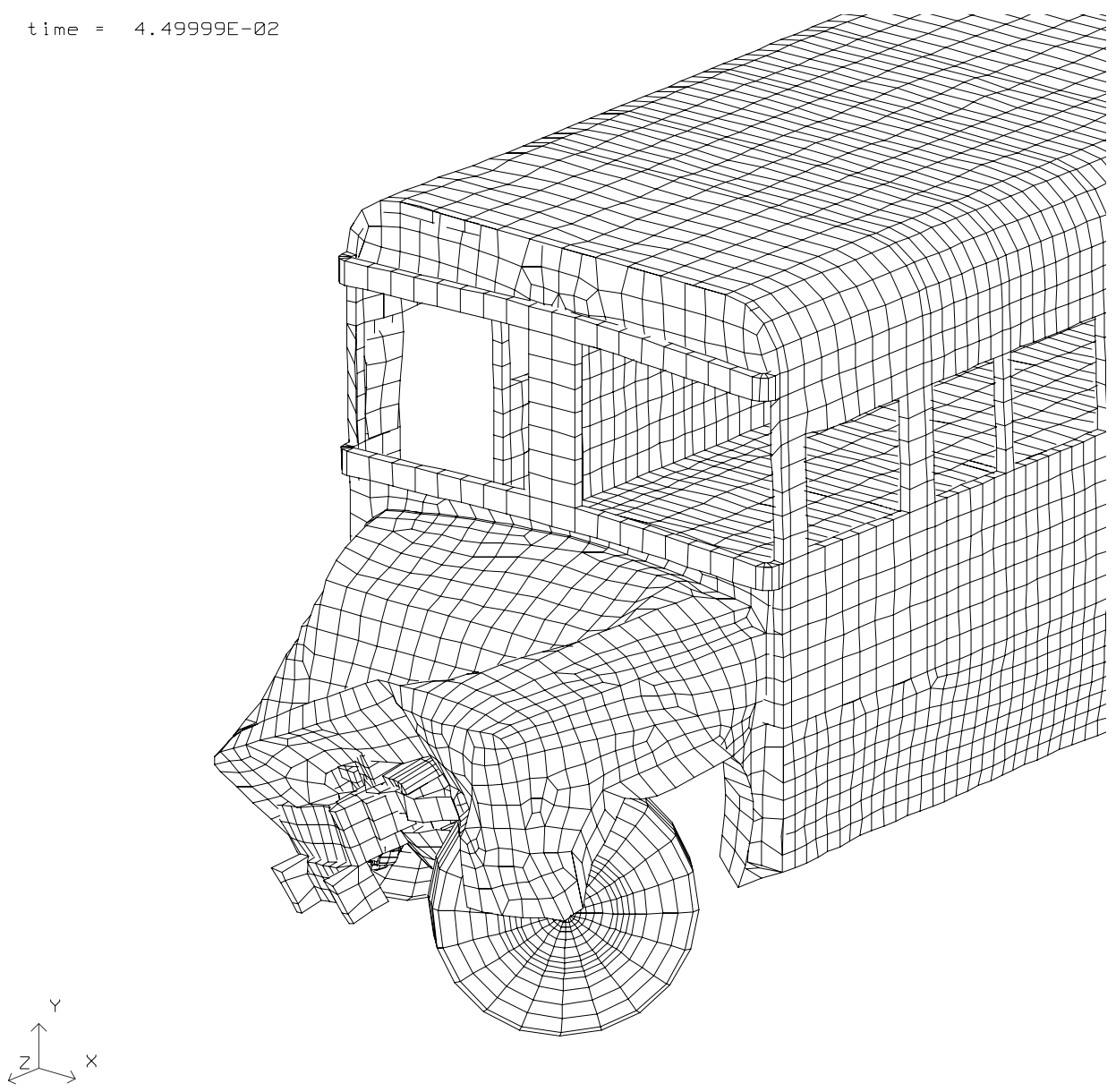

Fig 5.93 Front view at time $=45$ msec of the bus subjected to an offset frontal impact with Hughes-Liu SR thin shells.

Figure 5.94 shows the front view at time $=54 \mathrm{msec}$ of the chassis of the bus subjected to an offset frontal impact with Hughes-Liu SR thin shell elements. The frame absorbs the maximum internal energy, which is the same for the case without Hughes-Liu SR thin shell elements. The global velocity reduces to $4.1 \mathrm{~m} / \mathrm{sec}$ and tends to continue as a constant thereafter, which shows that the decelerations are zero from this time state and the solution is approaching a steady state. This can be seen from the graph in Figure 
5.112. The bending of the frame after the second engine mounting cross member is illustrated. The front end of the frame moves in the negative $\mathrm{Y}$ direction after hitting the rigid wall in this case, but in the case of an offset frontal impact without Hughes-Liu SR thin shells, the front end of the frame moves in the positive $\mathrm{Y}$ direction. This can be seen by comparing Figures 5.94 and 5.37.

time $=5.39999 \mathrm{E}-02$

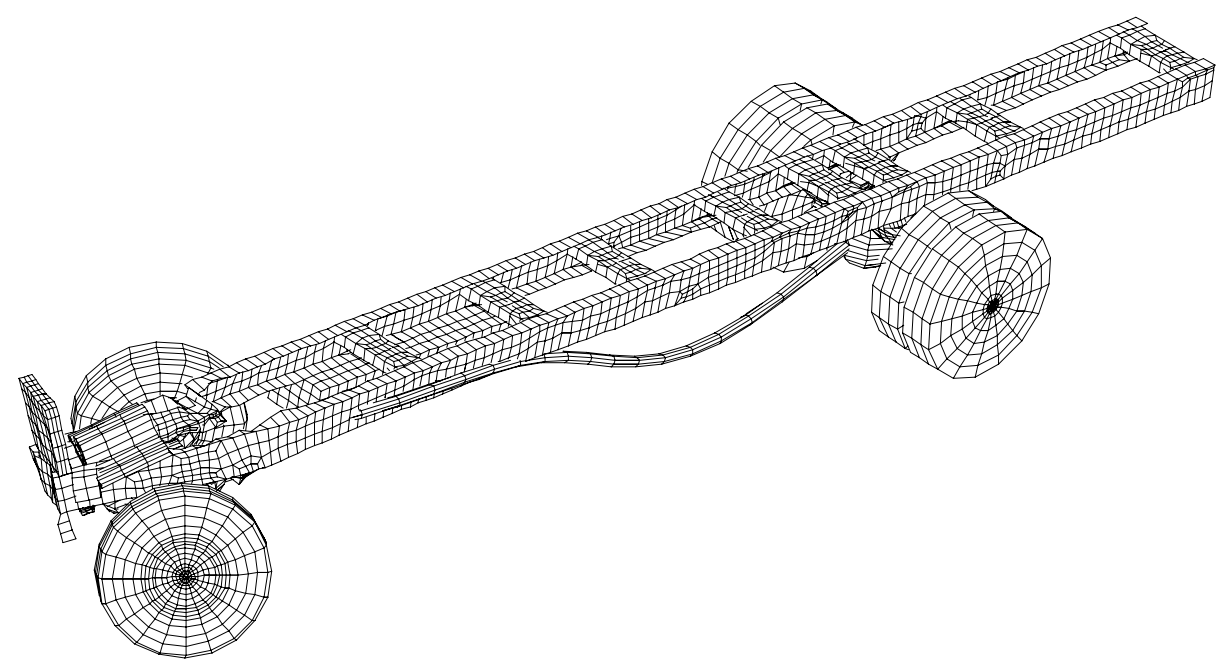

Fig 5.94 Front view at time $=\mathbf{5 4}$ msec of the bus subjected to an offset frontal impact with Hughes-Liu SR thin shells.

Figures 5.95 and 5.96 show the side view and top view at time $=54 \mathrm{msec}$ of the bus subjected to an offset frontal impact with Hughes-Liu SR thin shell elements. The rotation of the front axle due to the offset in the principal direction of the impact force is 
illustrated. The bending of the propeller shaft is also seen in the figure. The side view shows the deformation on only one side of the hood due to an offset in the impact force.

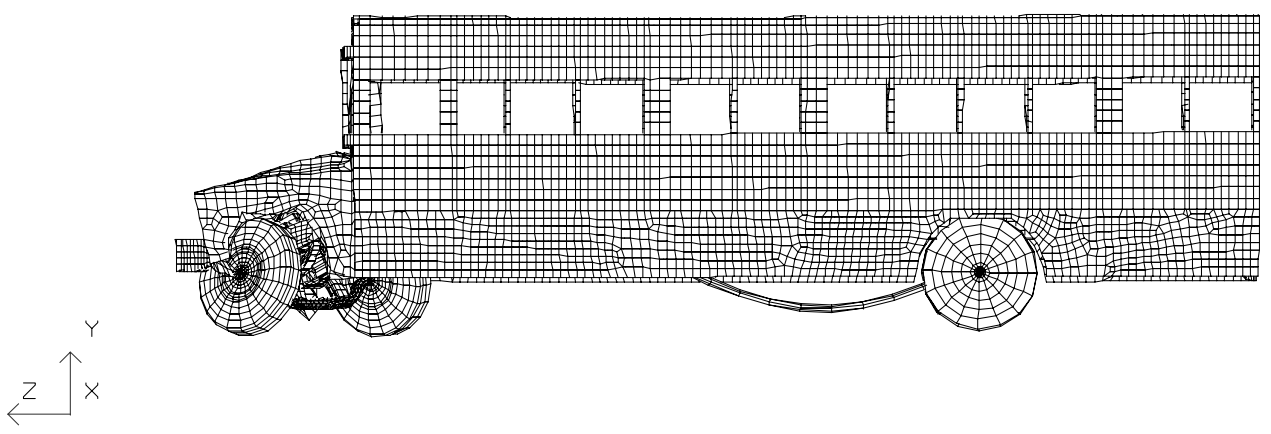

Fig 5.95 Side view at time $=\mathbf{5 4}$ msec of the bus subjected to an offset frontal impact with Hughes-Liu SR thin shells.
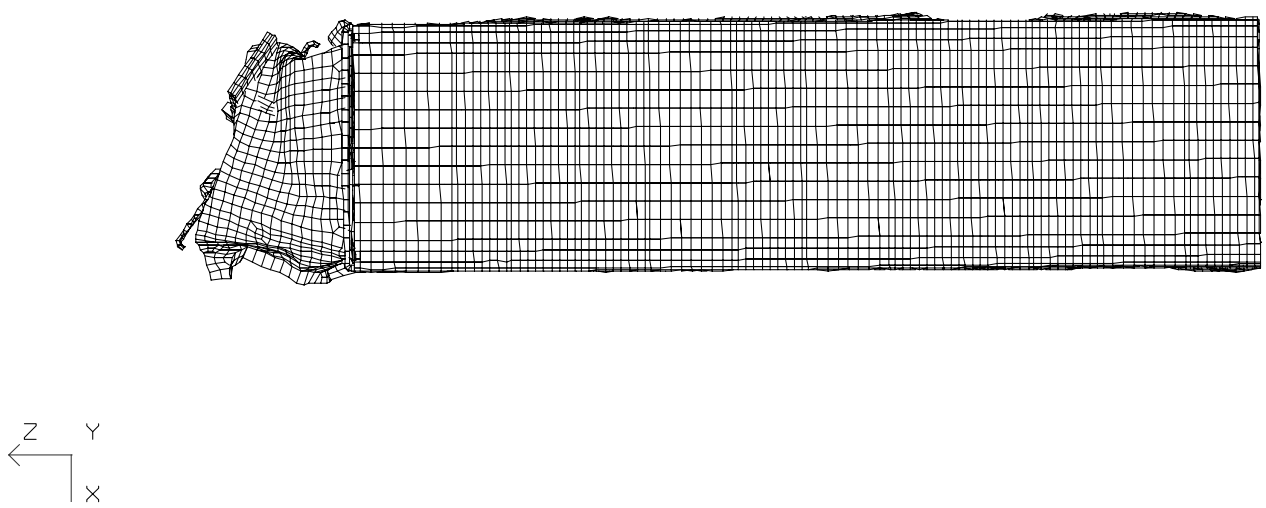

Fig 5.96 Top view at time $=54$ msec of the bus subjected to an offset frontal impact with Hughes-Liu SR thin shells. 
Figure 5.97 shows the relative displacement between the right wheel and the left wheel for an offset frontal impact with Hughes-Liu SR thin shell elements. The relative displacement continuously increases, which shows that the front axle rotates about the Y axis. The maximum relative displacement is $1.4 \mathrm{~m}$ in this case as compared to $0.65 \mathrm{~m}$ for an offset frontal impact without Hughes-Liu SR thin shell elements. This shows that the rotation of the front axle is higher in this case. Figure 5.98 shows the relative displacement between the front tire and the rear tire. The relative displacement continuously increases, which shows the pitching of the vehicle. The maximum relative displacement is $0.16 \mathrm{~m}$ in this case and this is higher than the case for an offset frontal impact without Hughes-Liu SR thin shell elements since the vehicle starts pitching only after $65 \mathrm{msec}$.

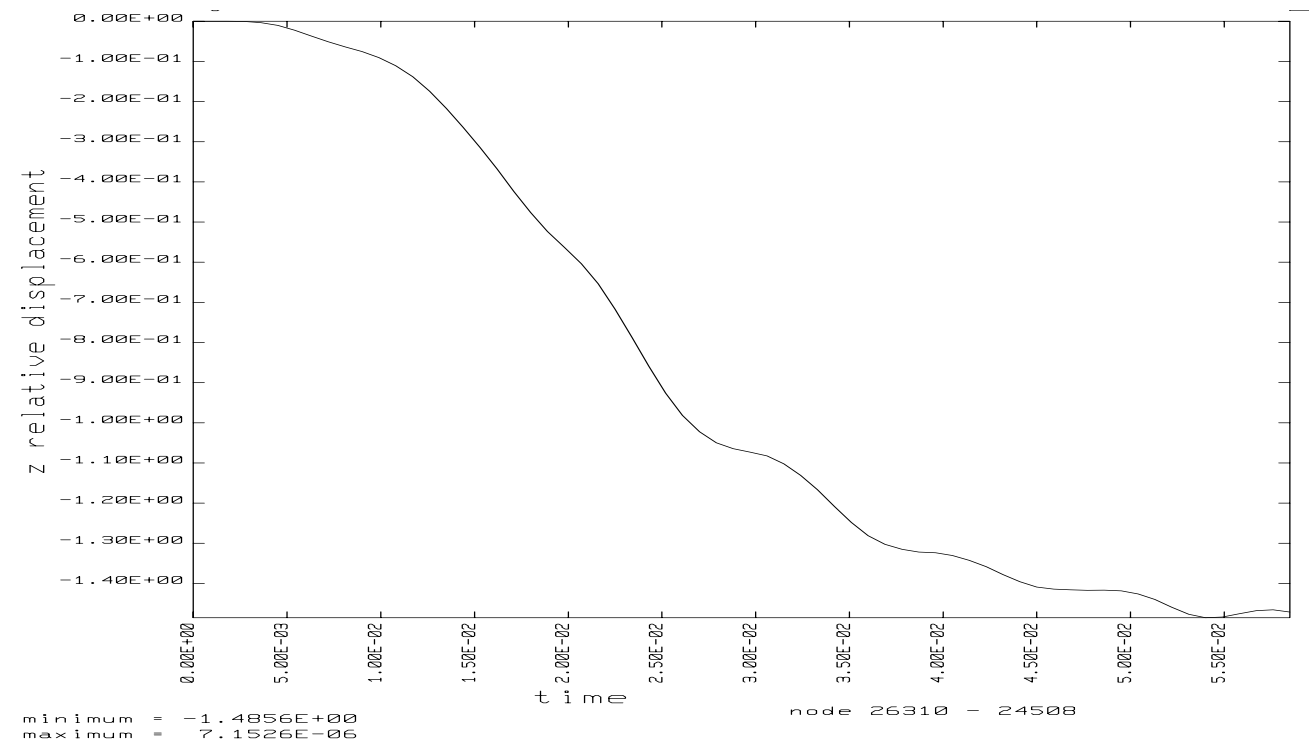

Fig 5.97 Relative displacement between the right wheel and the left wheel (axle rotation) for an offset frontal impact with Hughes-Liu SR thin shell elements. 


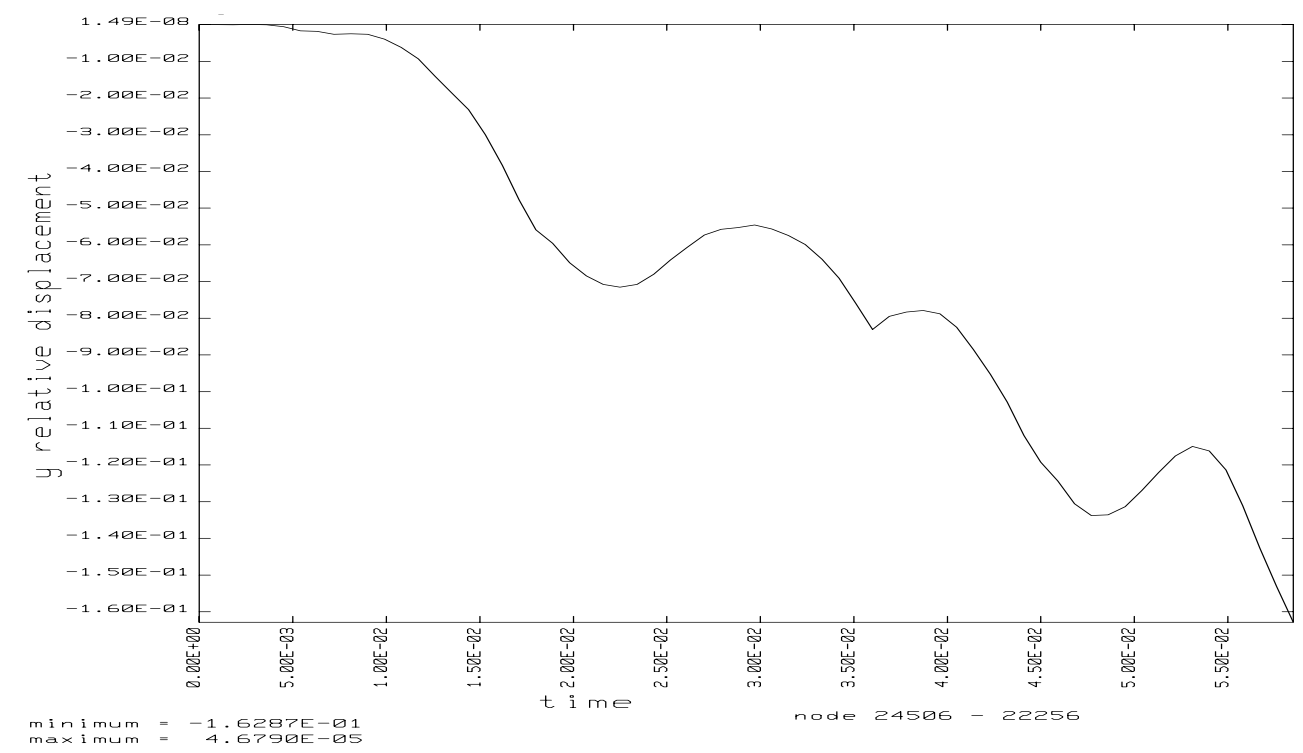

Fig 5.98 Relative displacement between the front tires and the rear tires (pitching) for an offset frontal impact with Hughes-Liu SR thin shell elements.

Figures 5.99, 5.100, and 5.101 show the $\mathrm{Z}$ displacement for the nodes in the front, middle, and rear of the vehicle subjected to an offset frontal impact with Hughes-Liu SR thin shell elements. The maximum displacement in the front, middle, and rear of the vehicle is the same, which shows that there is no relative displacement between the nodes in the three positions. This shows that the occupant area remains unaffected. However the nodes in the right, center, and left at the front of the vehicle behave differently as compared to the nodes in the other positions of the vehicle. Node 966 which lies in the right (door side) side of the vehicle, undergoes less deformation compared to the nodes in the center and left side. This is because of the impact only on the right side of the vehicle. When the maximum displacement of the vehicle in this case is compared to the maximum displacement for an offset frontal impact without Hughes-Liu SR thin shell elements, it can be seen that the maximum value remains the same in both the cases. This shows that there is no difference in the values of the displacement in the $\mathrm{Z}$ direction. 


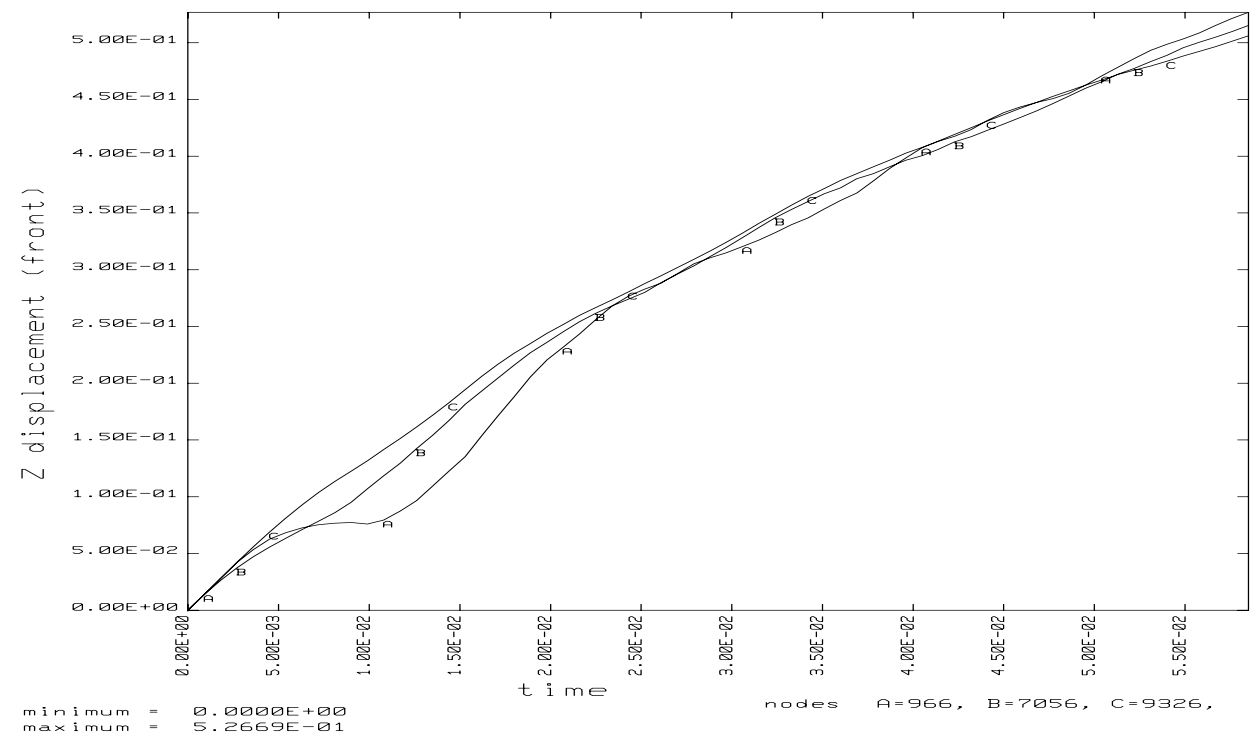

Fig 5.99 $\mathrm{Z}$ displacement for the nodes in the front of the vehicle for an offset frontal impact with Hughes-Liu SR thin shells.

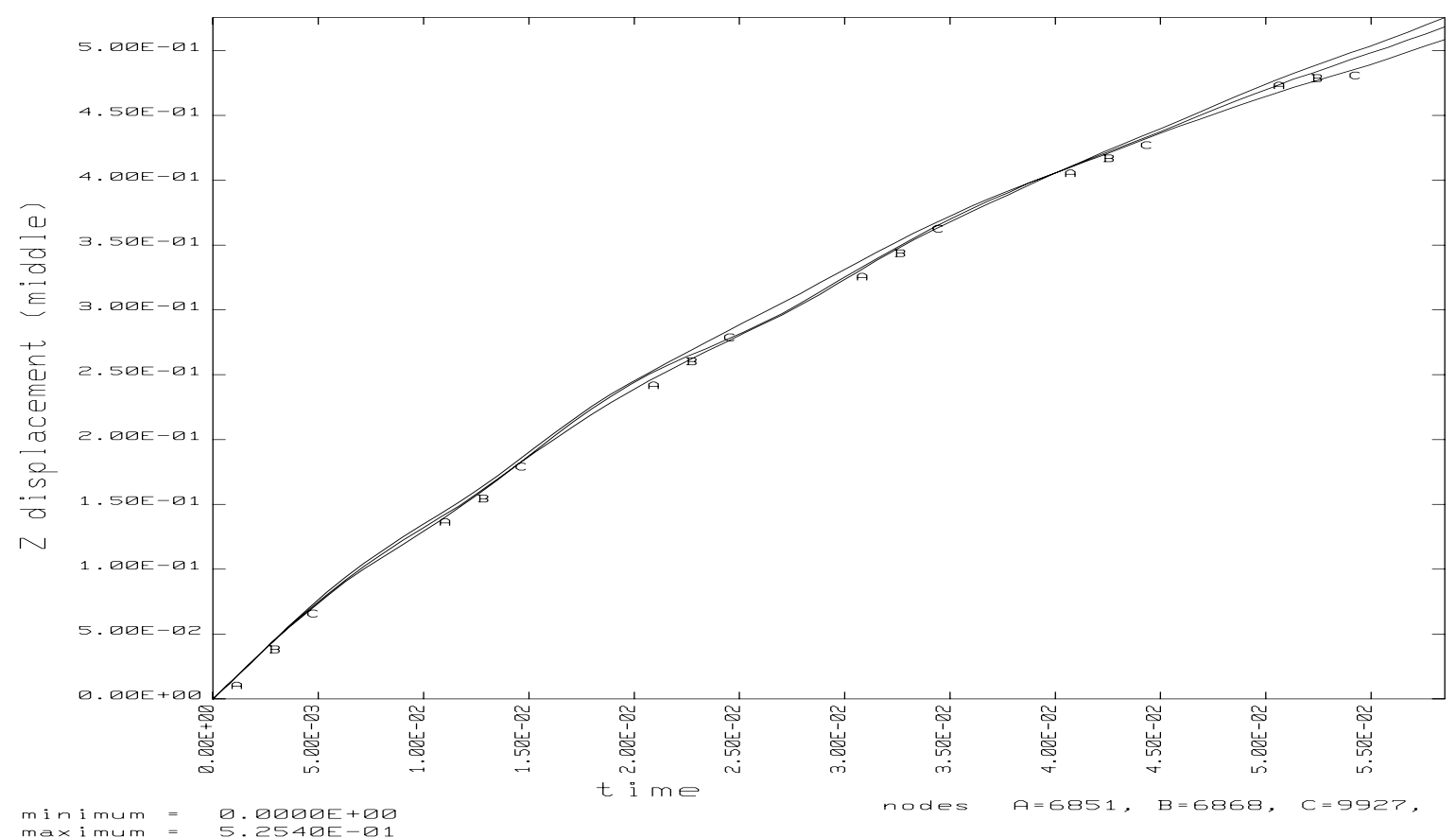

Fig 5.100 $\mathrm{Z}$ displacement for the nodes in the middle of the vehicle for an offset frontal impact with Hughes-Liu SR thin shells. 


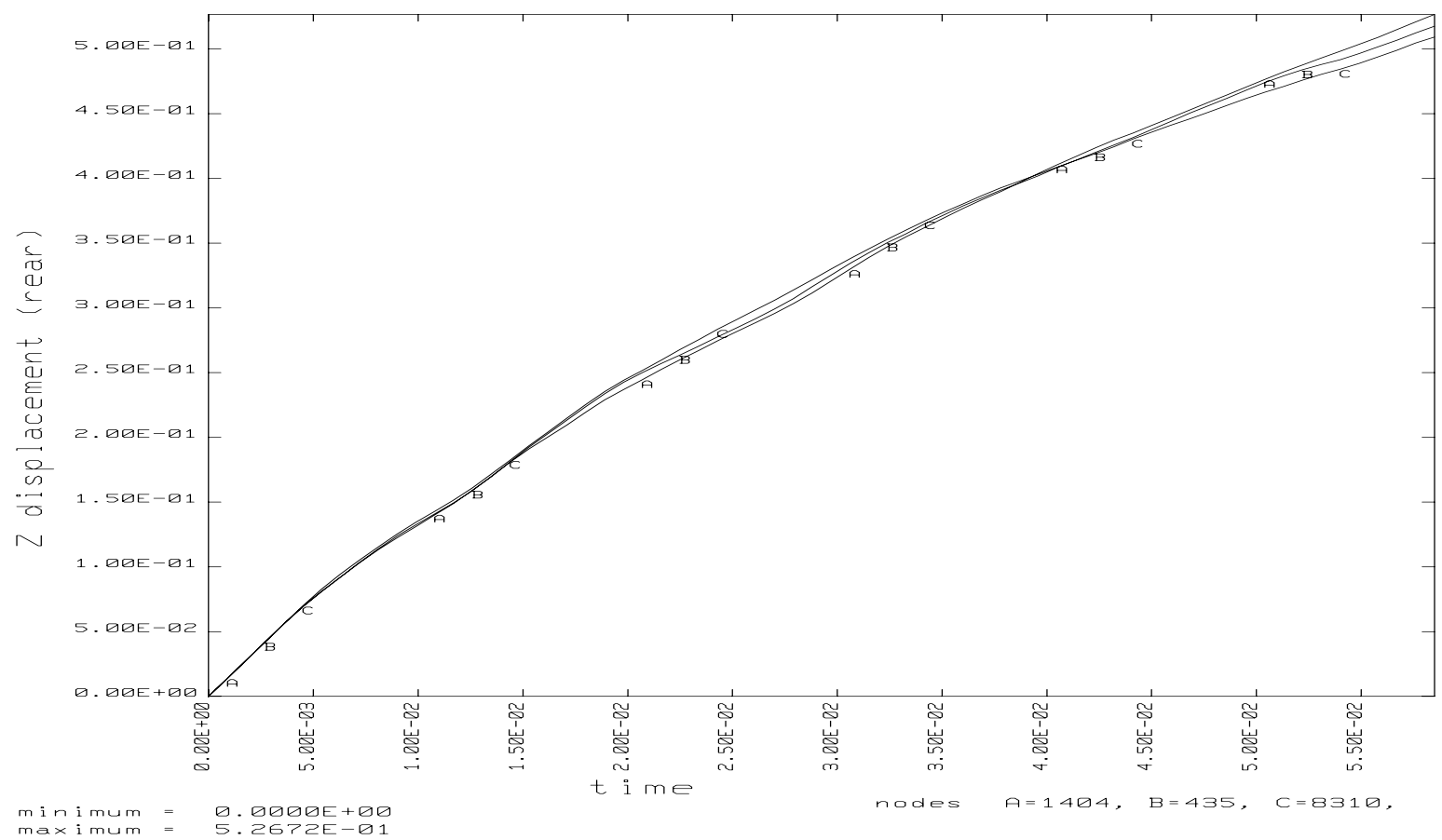

Fig 5.101 $\mathrm{Z}$ displacement for the nodes in the rear of the vehicle for an offset frontal impact with Hughes-Liu SR thin shells.

Figures 5.102, 5.103, and 5.104 show the $\mathrm{Z}$ velocity for the nodes in the front, middle, and rear of the vehicle. From Figure 5.102 it can be seen that node 966 that lies on the right (door side) side of the vehicle reaches zero velocity before $10 \mathrm{msec}$, since it lies on the impact side of the body structure. The velocity of the nodes in the middle and the rear of the vehicle remain the same, which shows that there is no relative deformation between them and hence no protrusion into the occupant area. The velocity of these points tend to $6 \mathrm{~m} / \mathrm{sec}$ and stay constant. The values of the velocity of the nodes at these points in this case is the same as the case of an offset frontal impact without Hughes-Liu SR thin shell elements. 


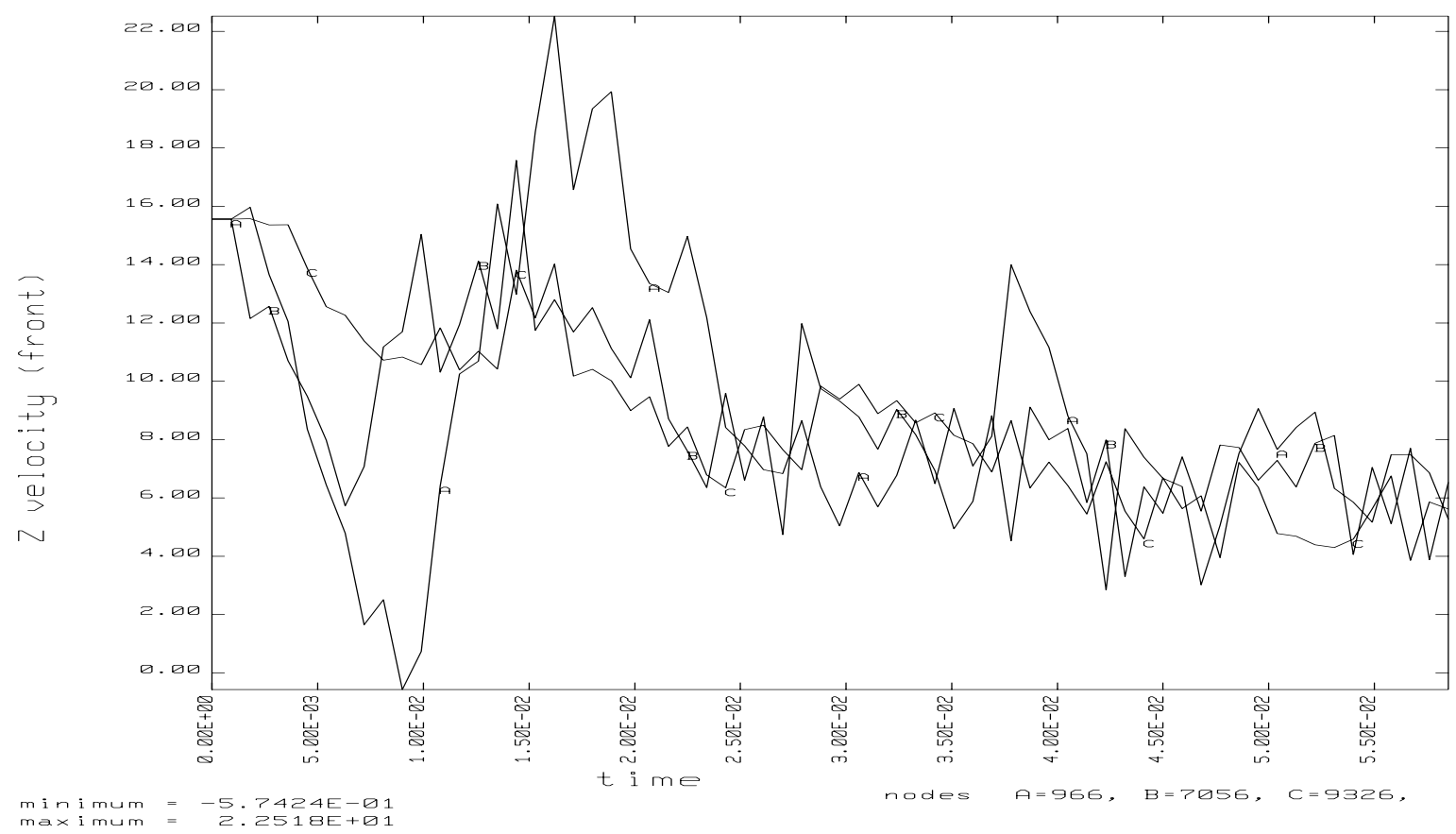

Fig 5.102 $\mathrm{Z}$ velocity for the nodes in the front of the vehicle for an offset frontal impact with Hughes-Liu SR thin shells.

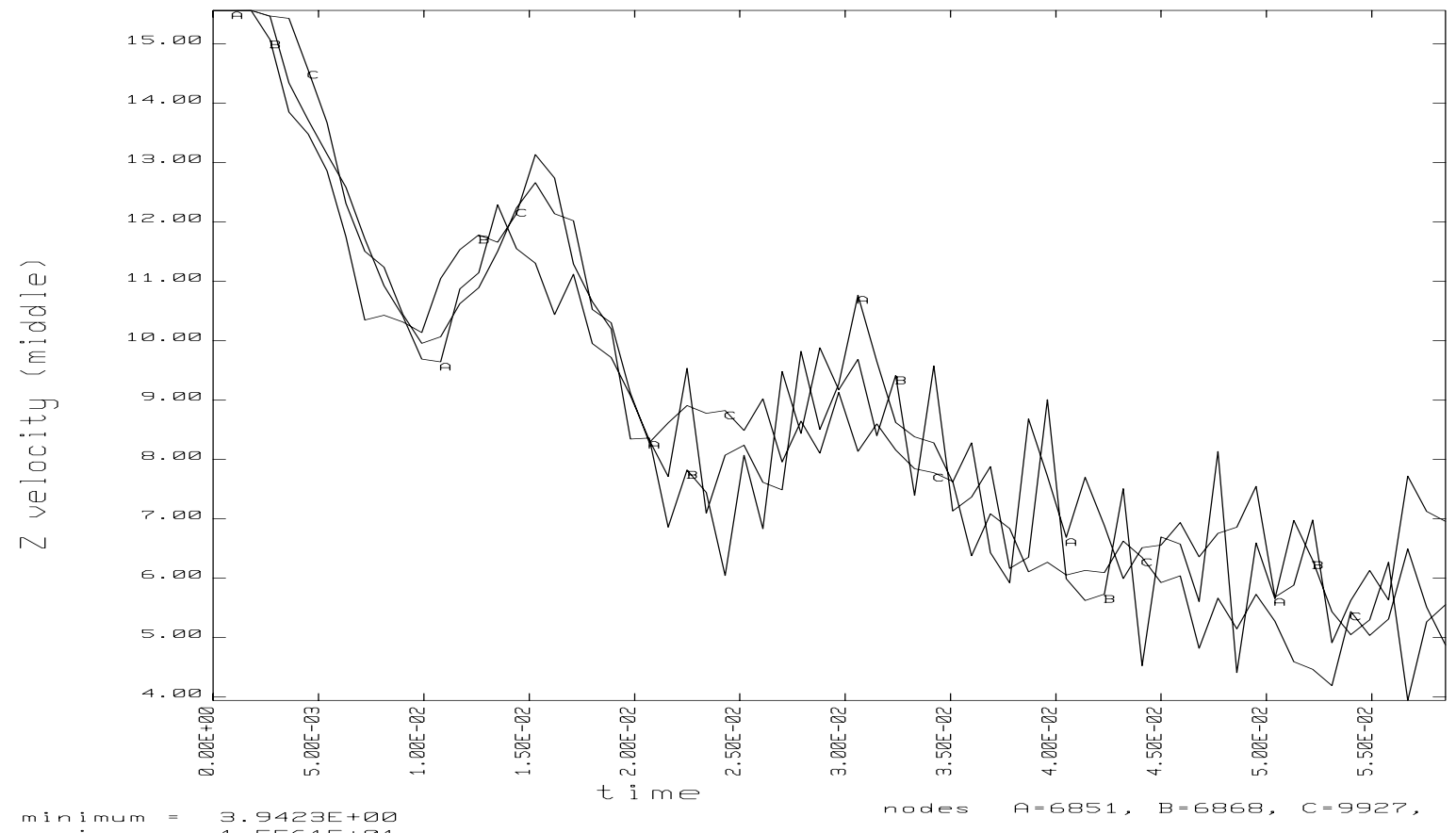

Fig 5.103 $\mathrm{Z}$ velocity for the nodes in the middle of the vehicle for an offset frontal impact with Hughes-Liu SR thin shells. 


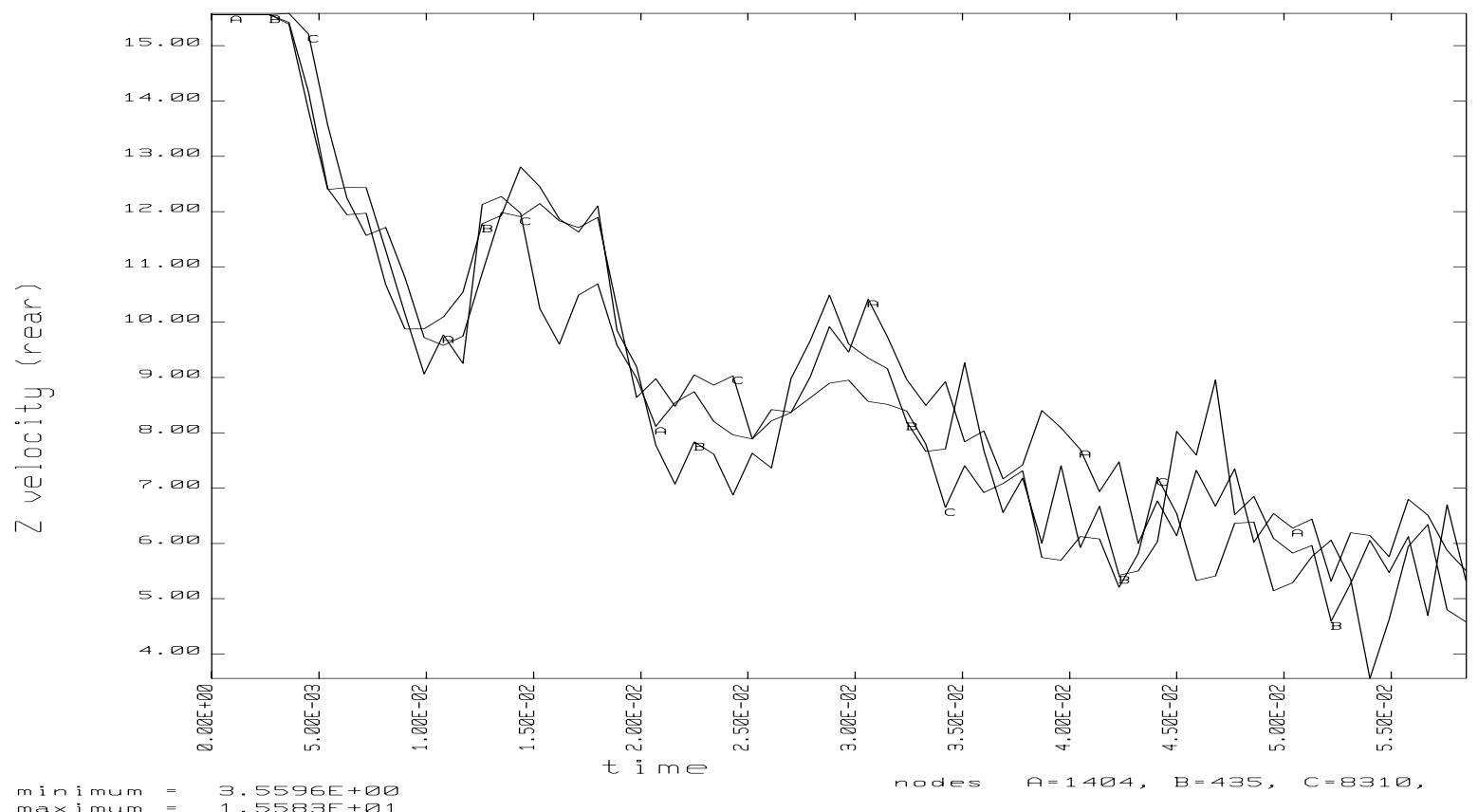

Fig 5.104 $\mathrm{Z}$ velocity for the nodes in the rear of the vehicle for an offset frontal impact with Hughes-Liu SR thin shells.

Figures 5.105, 5.106, and 5.107 show the $\mathrm{Z}$ acceleration for the nodes in the front, middle, and rear of the vehicle. From Figure 5.105, it can be seen that the maximum deceleration of node 966 is higher compared to the other nodes since it lies on the impact side of the body structure. From Figure 5.106, it can be seen that the deceleration of the node at the center in the middle of the body structure is higher than the nodes on the right and the left side. The maximum values of the deceleration in this case are higher compared to the case of an offset frontal impact without Hughes-Liu SR thin shell elements. 


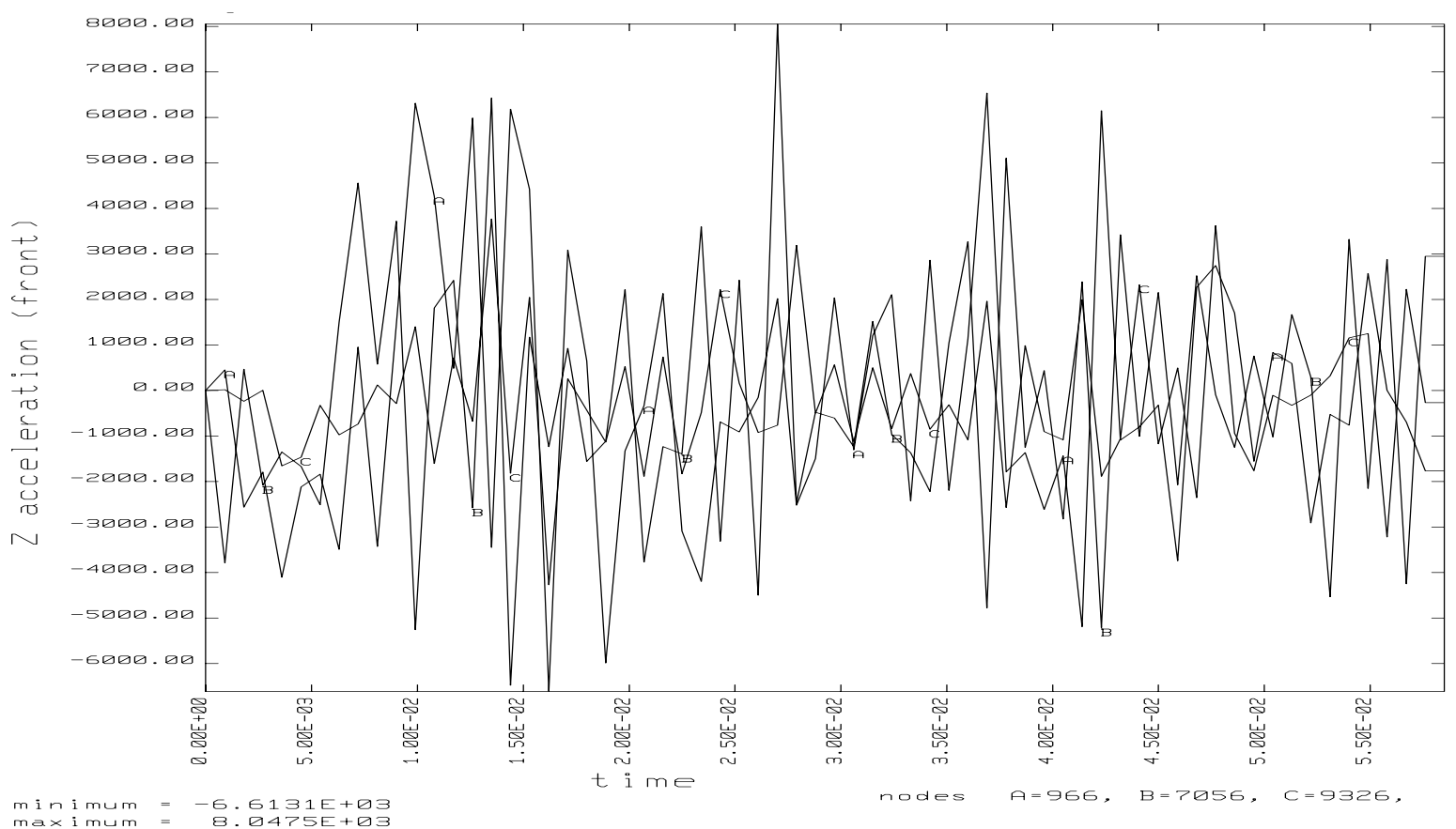

Fig 5.105 $\mathrm{Z}$ acceleration for the nodes in the front of the vehicle for an offset frontal impact with Hughes-Liu SR thin shells.

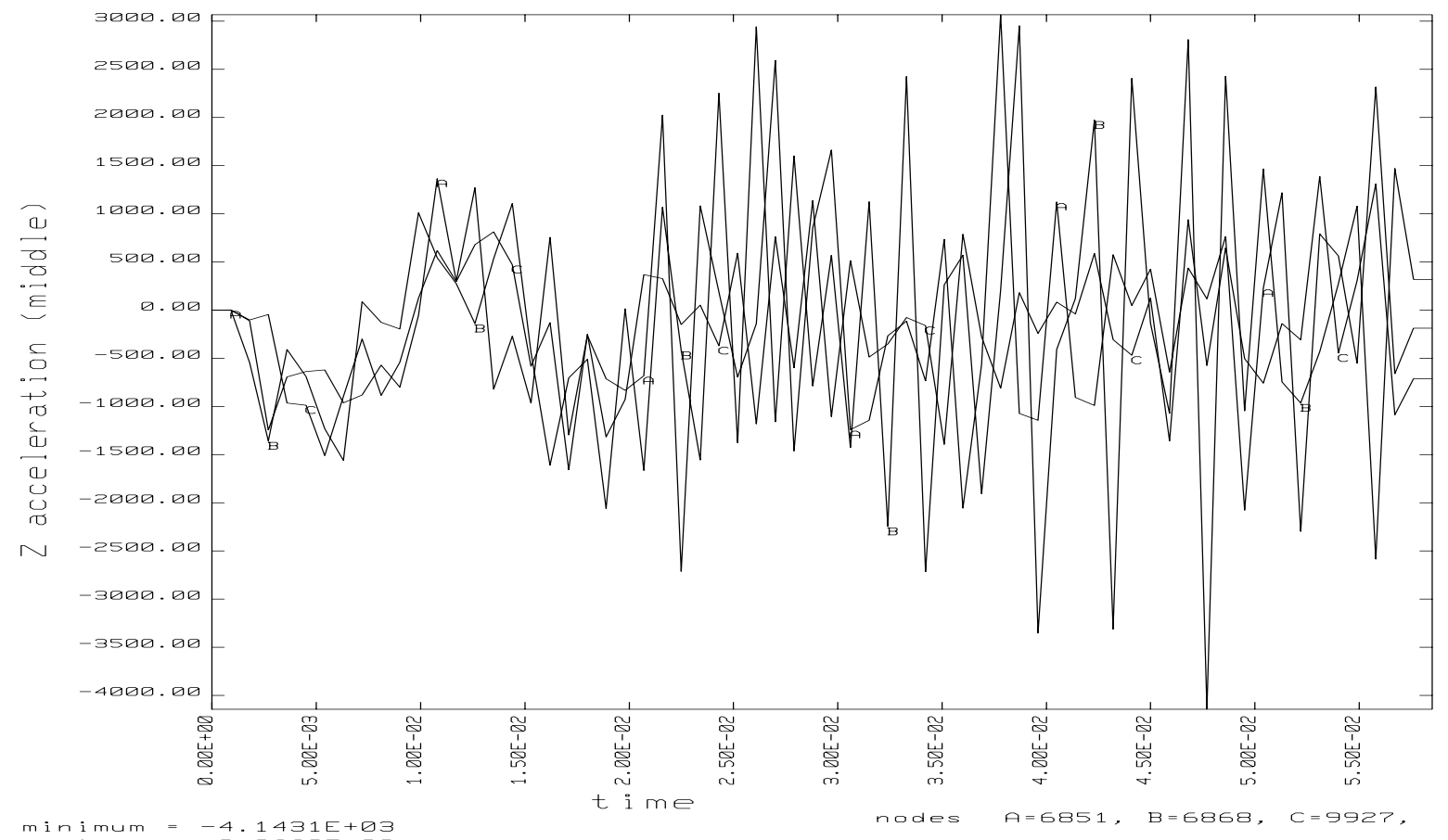

Fig 5.106 $\mathrm{Z}$ acceleration for the nodes in the middle of the vehicle for an offset frontal impact with Hughes-Liu SR thin shells. 


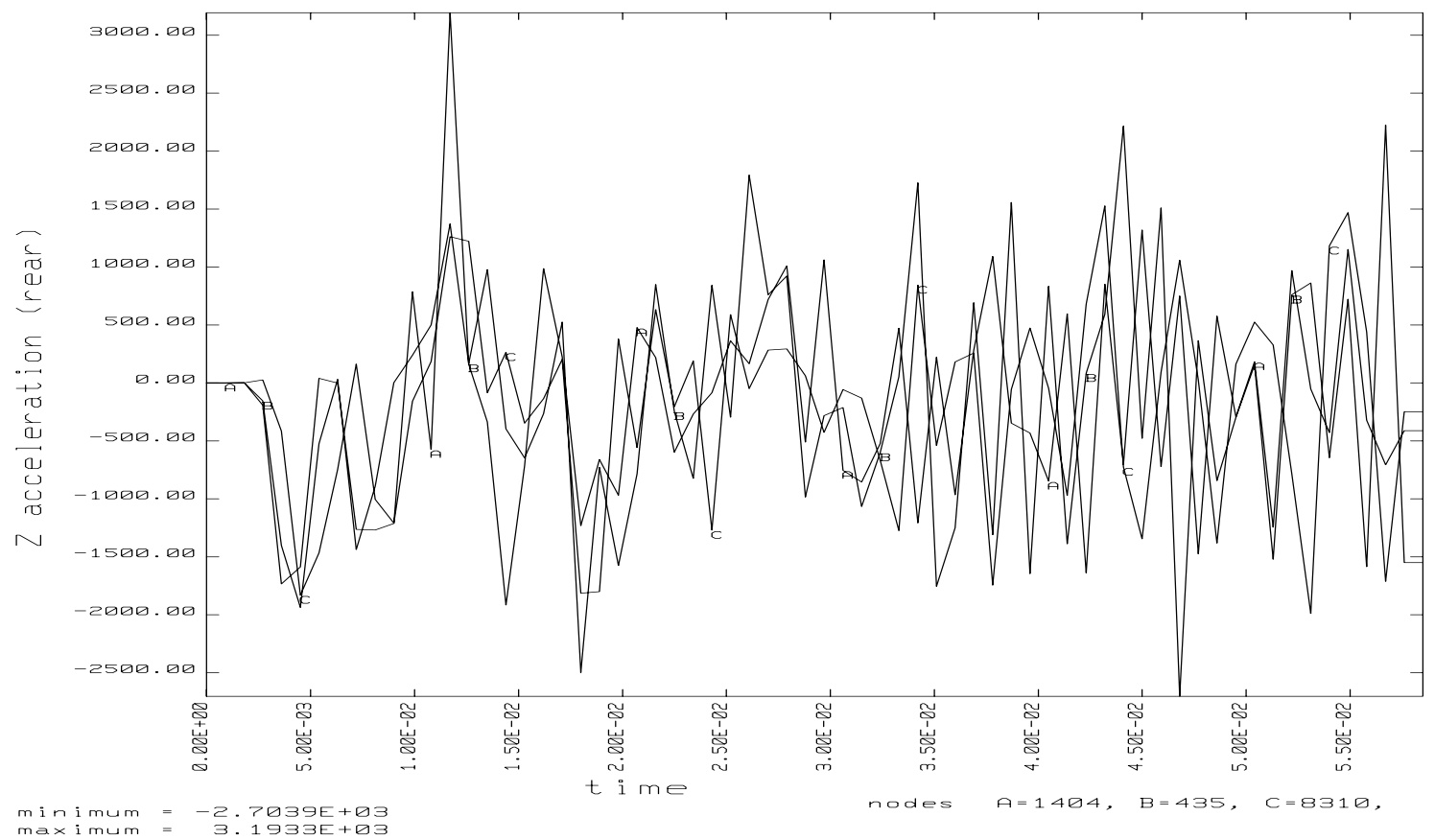

Fig 5.107 $\mathrm{Z}$ acceleration for the nodes in the rear of the vehicle for an offset frontal impact with Hughes-Liu SR thin shells.

Figure 5.108 shows the material rigid body velocity of parts for an offset frontal impact with Hughes-Liu SR thin shell elements. From the graph it can be seen that the velocities of the parts tend to become a constant after $40 \mathrm{msec}$. 


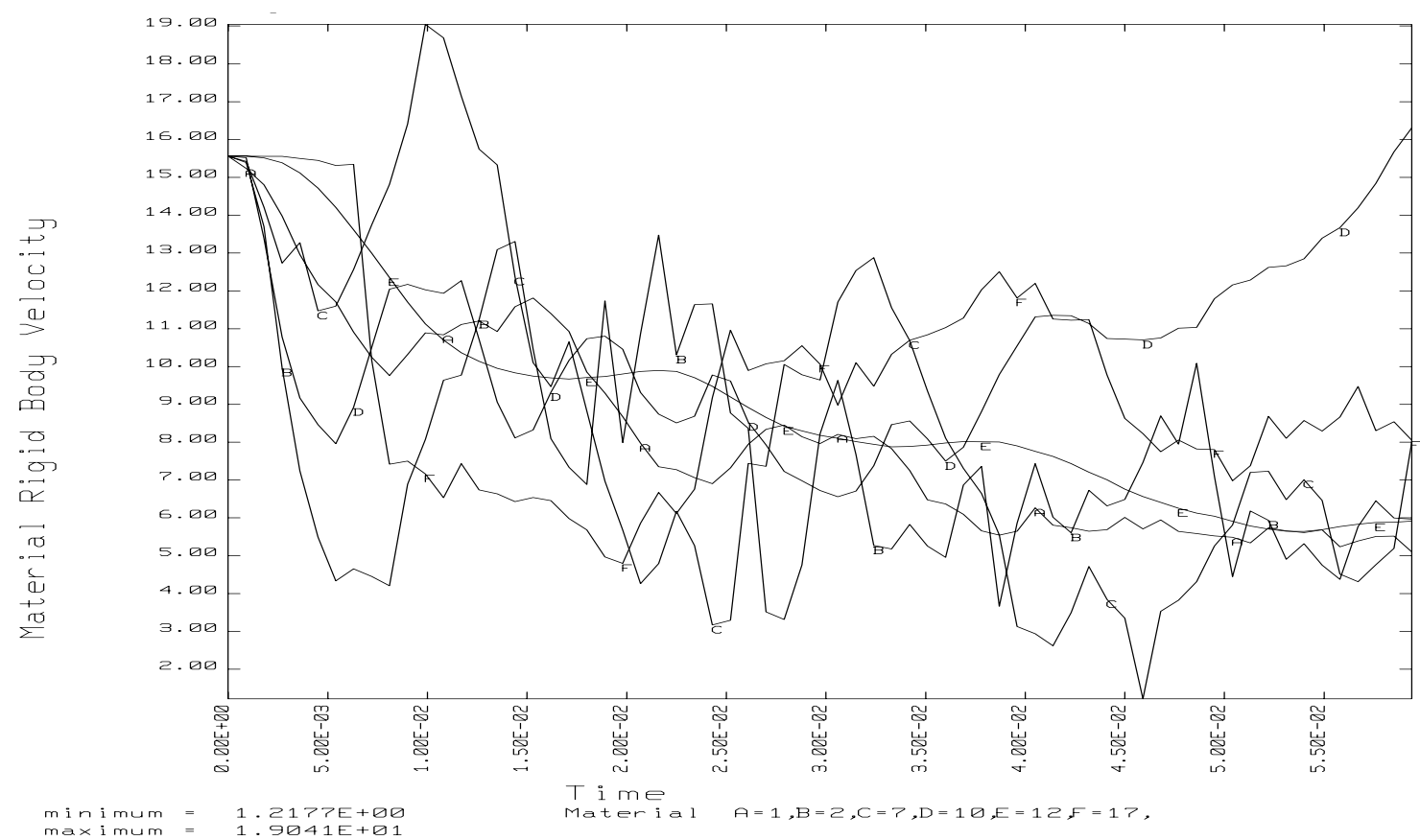

Fig 5.108 Material rigid body velocity of parts for an offset frontal impact with Hughes-Liu SR thin shells.

Figures 5.109, 5.110, 5.111, and 5.112 show the X, Y, Z, and total rigid body velocity for the bus subjected to an offset frontal impact with Hughes-Liu SR thin shell elements. From Figure 5.109 it can be seen that the $\mathrm{X}$ rigid body velocity of the bus is negligible. This shows that there is no yaw in the vehicle behavior. From the Figure 5.110 it can be seen that the $\mathrm{Y}$ rigid body velocity of the bus is about a tenth of the $\mathrm{Z}$ rigid body velocity. This shows the pitching of the vehicle. The $\mathrm{Z}$ rigid body velocity decreases from $15.5 \mathrm{~m} / \mathrm{sec}$ and tends to be a constant after $55 \mathrm{msec}$ which shows the end of the solution. The total rigid body velocity is the resultant of the $\mathrm{X}, \mathrm{Y}$, and $\mathrm{Z}$ rigid body velocities. 


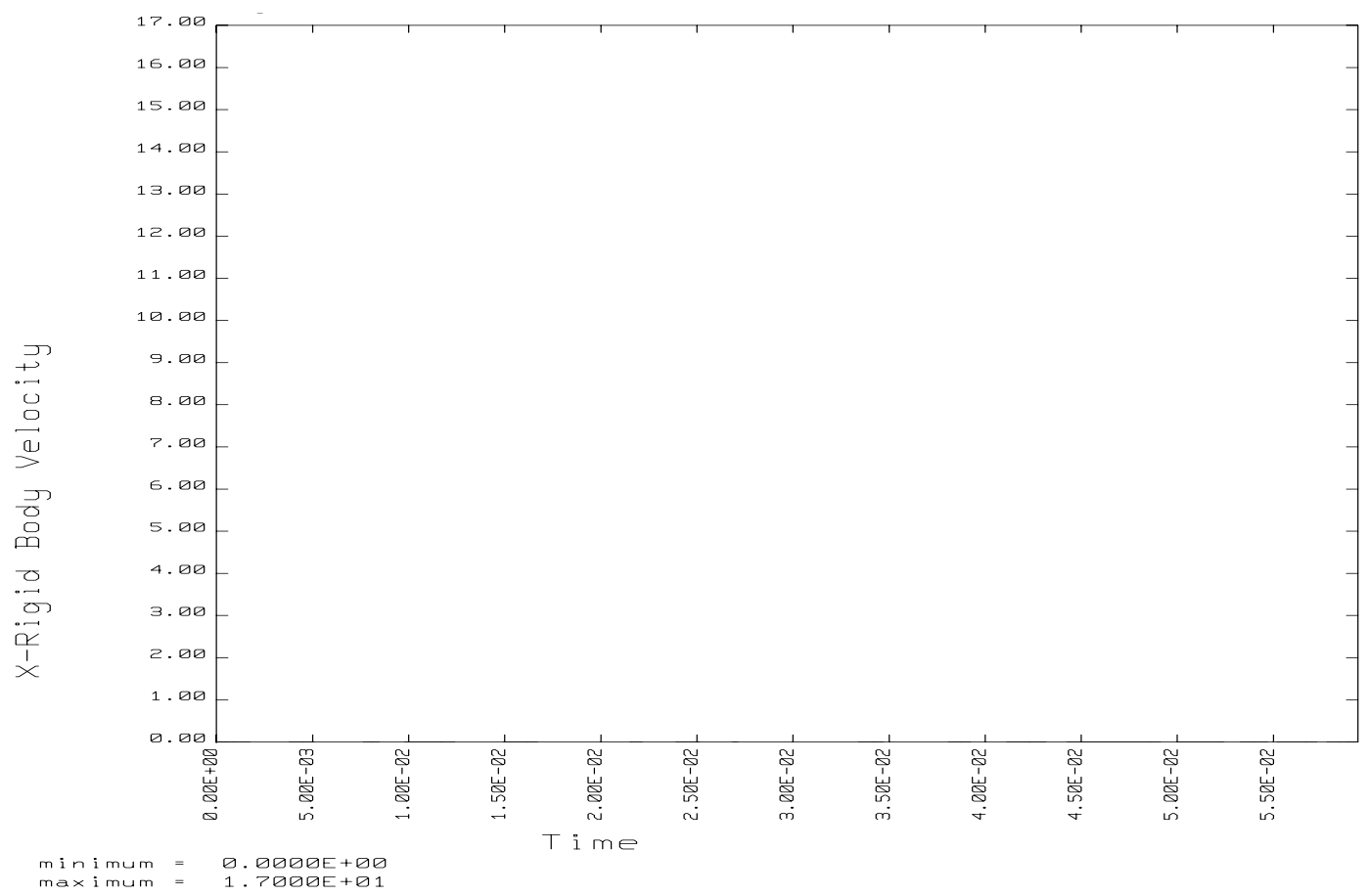

Fig 5.109 Global X rigid body velocity of the bus for an offset frontal impact with Hughes-Liu SR thin shells.

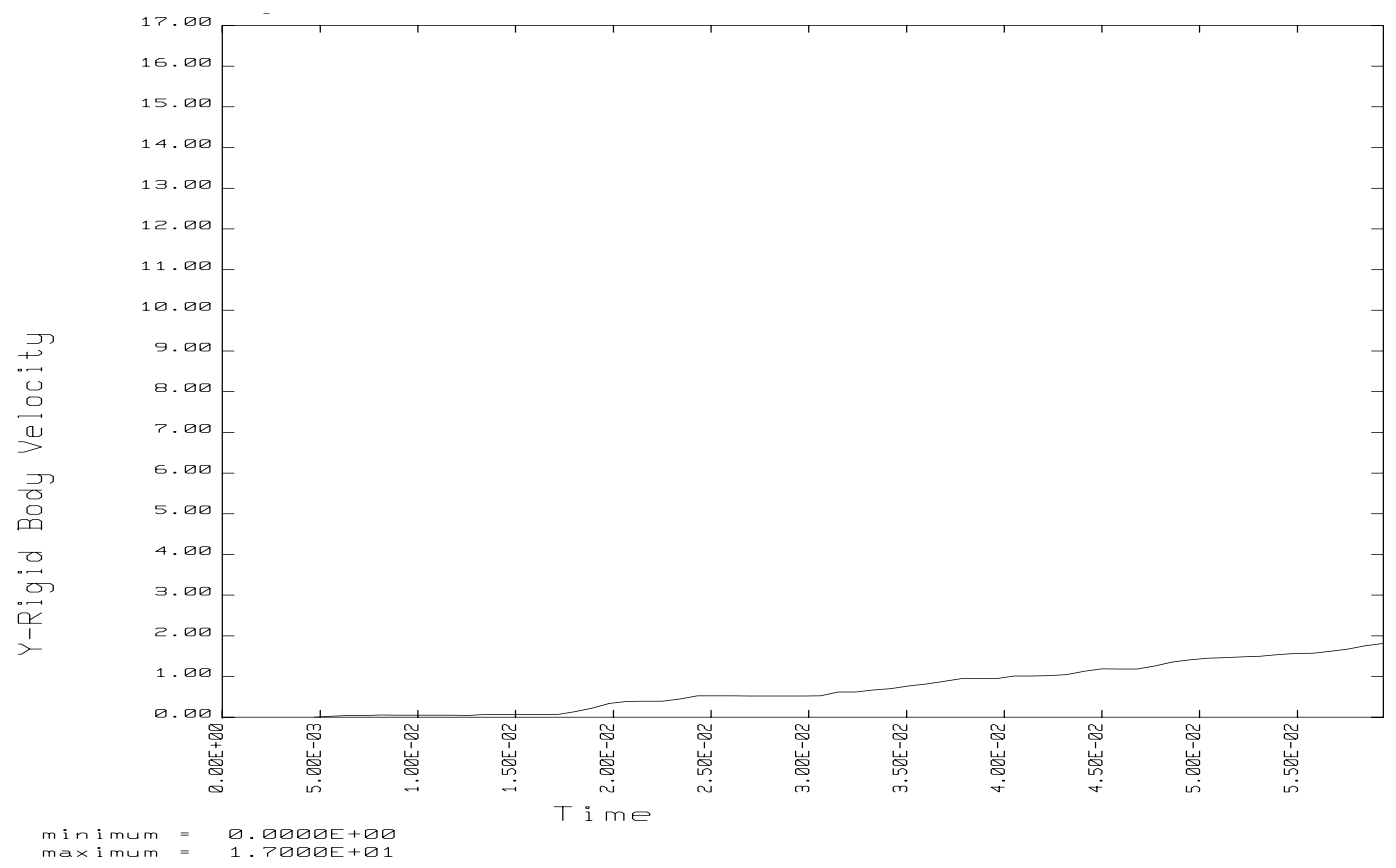

Fig 5.110 Global Y rigid body velocity of the bus for an offset frontal impact with Hughes-Liu SR thin shells. 


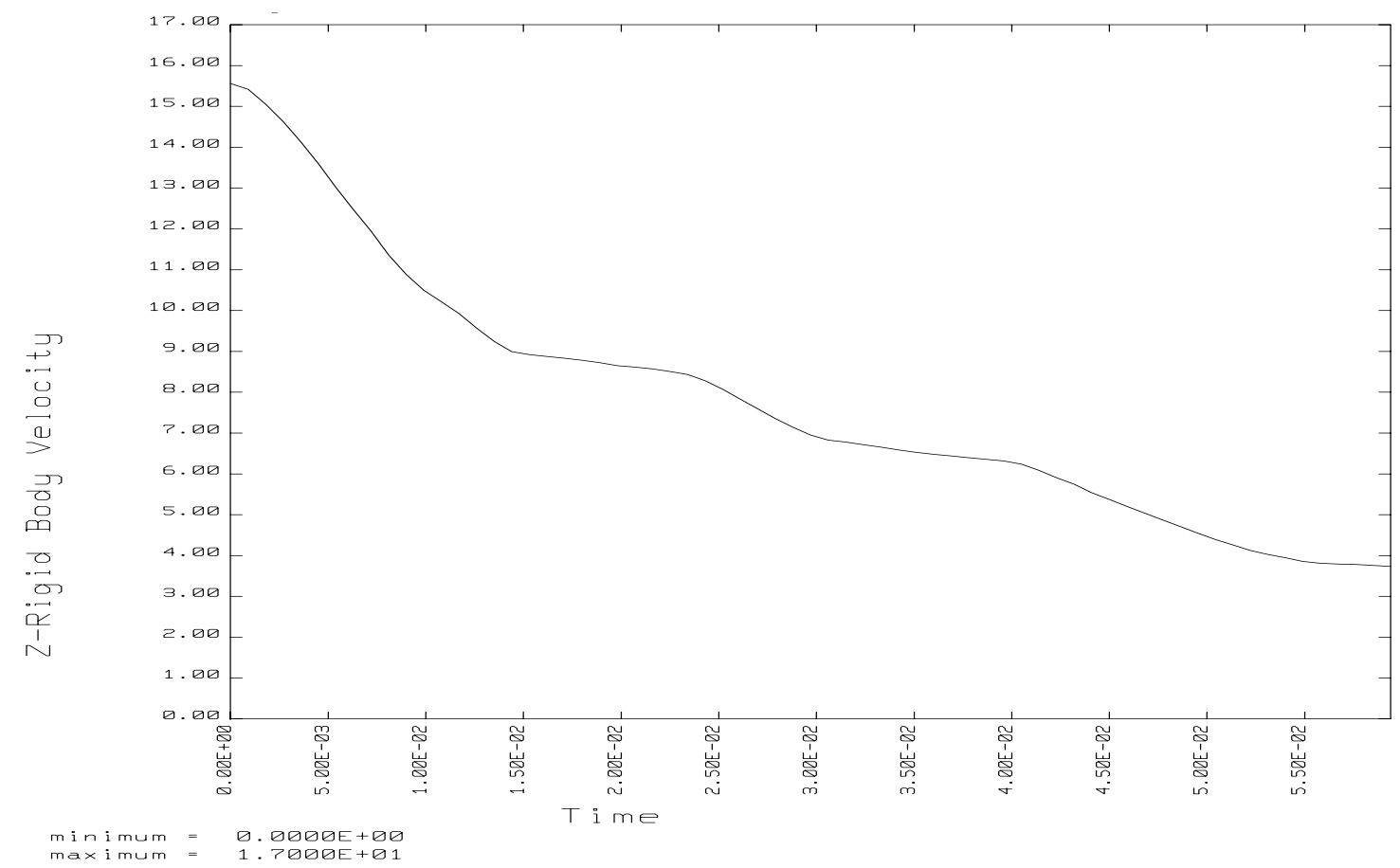

Fig 5.111 Global $Z$ rigid body velocity of the bus for an offset frontal impact with Hughes-Liu SR thin shells.

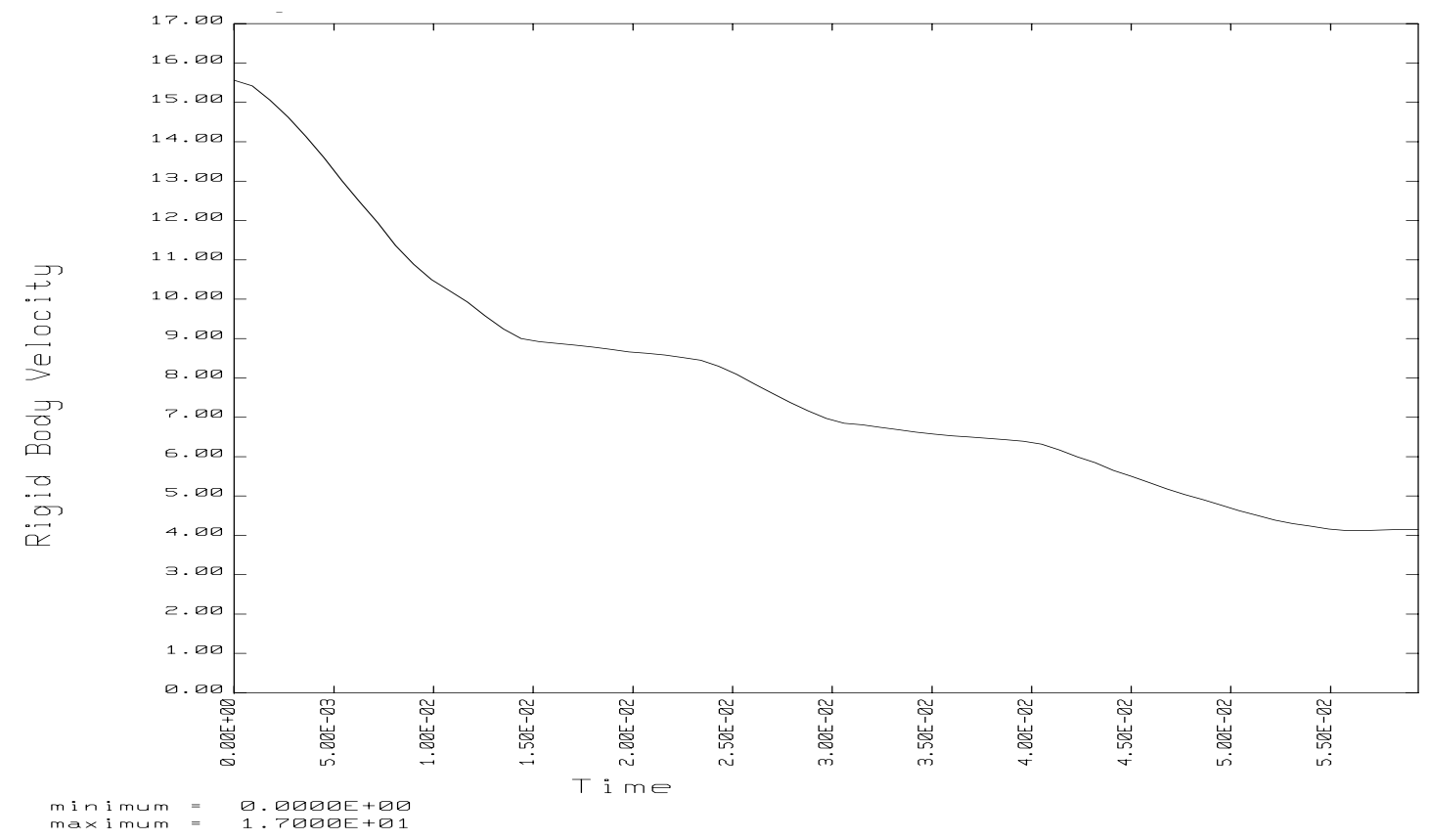

Fig 5.112 Global total rigid body velocity of the bus for an offset frontal impact with Hughes-Liu SR thin shells. 
Figure 5.113 shows the internal energy absorbed by various parts during an offset frontal impact with Hughes-Liu SR thin shell elements. The frame absorbs the maximum internal energy since it buckles after the second engine mounting cross member. The internal energy absorbed by the parts in this case is higher compared to the case of an offset frontal impact without Hughes-Liu SR thin shell elements because of the absence of hourglassing energy. In this case, the front axle absorbs more energy than the case of an offset frontal impact without Hughes-Liu SR thin shell elements. Figure 5.114 shows the internal, kinetic, and total energies of the system. The total energy remains constant which shows that the energy is conserved during the process. The internal and the kinetic energies sum up to the total energy because of the absence of the hourglassing energy. Figure 5.115 shows the pie chart for the internal energy distribution in a bus subjected to an offset frontal impact with Hughes-Liu SR thin shell elements. From the graph it can be seen that the frame remains a principal energy absorbing part in this case, as in the case of an offset frontal impact without Hughes-Liu SR thin shell elements. This can be seen by comparing the graphs in Figure 5.115 and Figure 5.56 at the end of the analysis. 


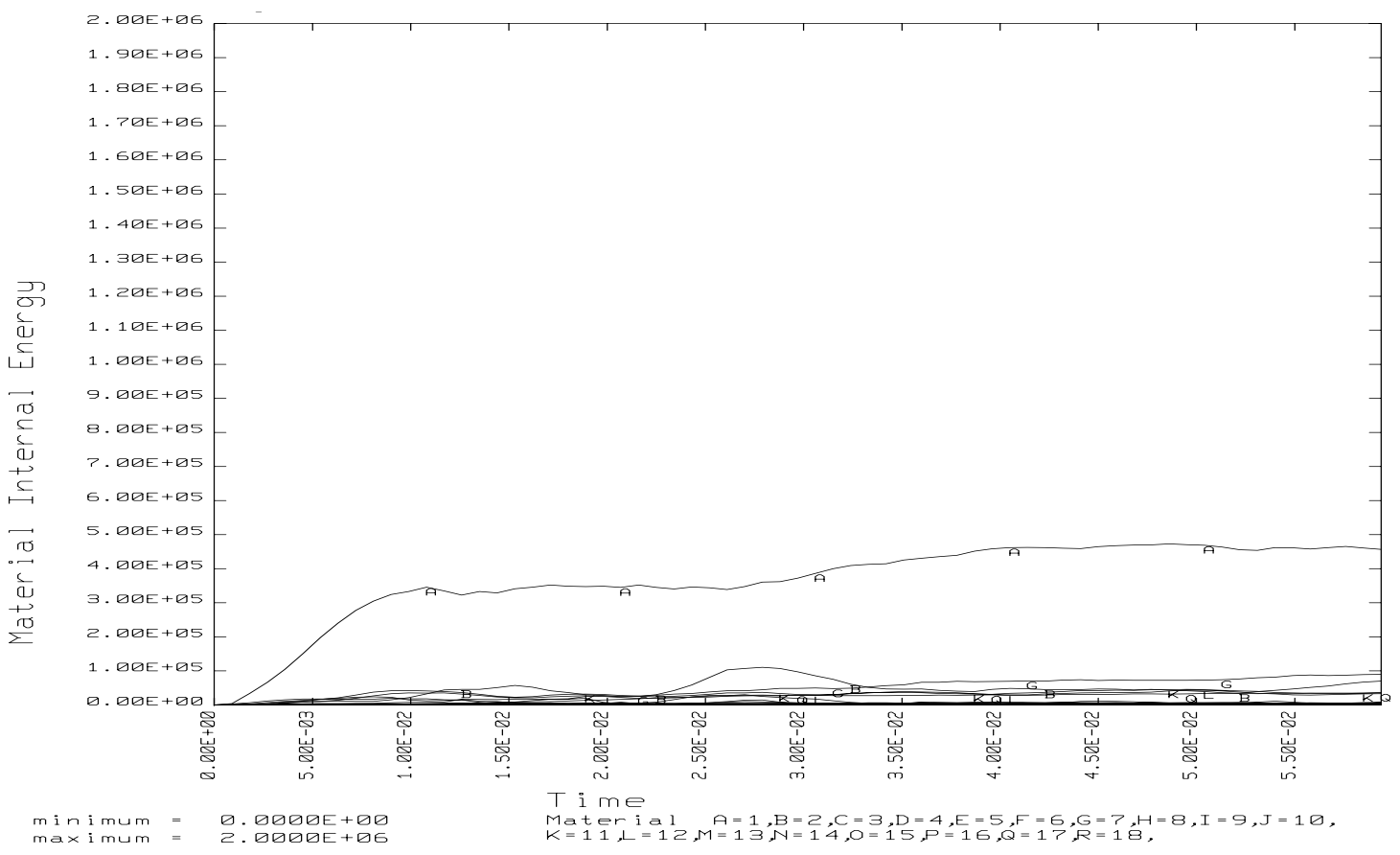

Fig 5.113 Material internal energy of parts for an offset frontal impact with Hughes-Liu SR thin shells.

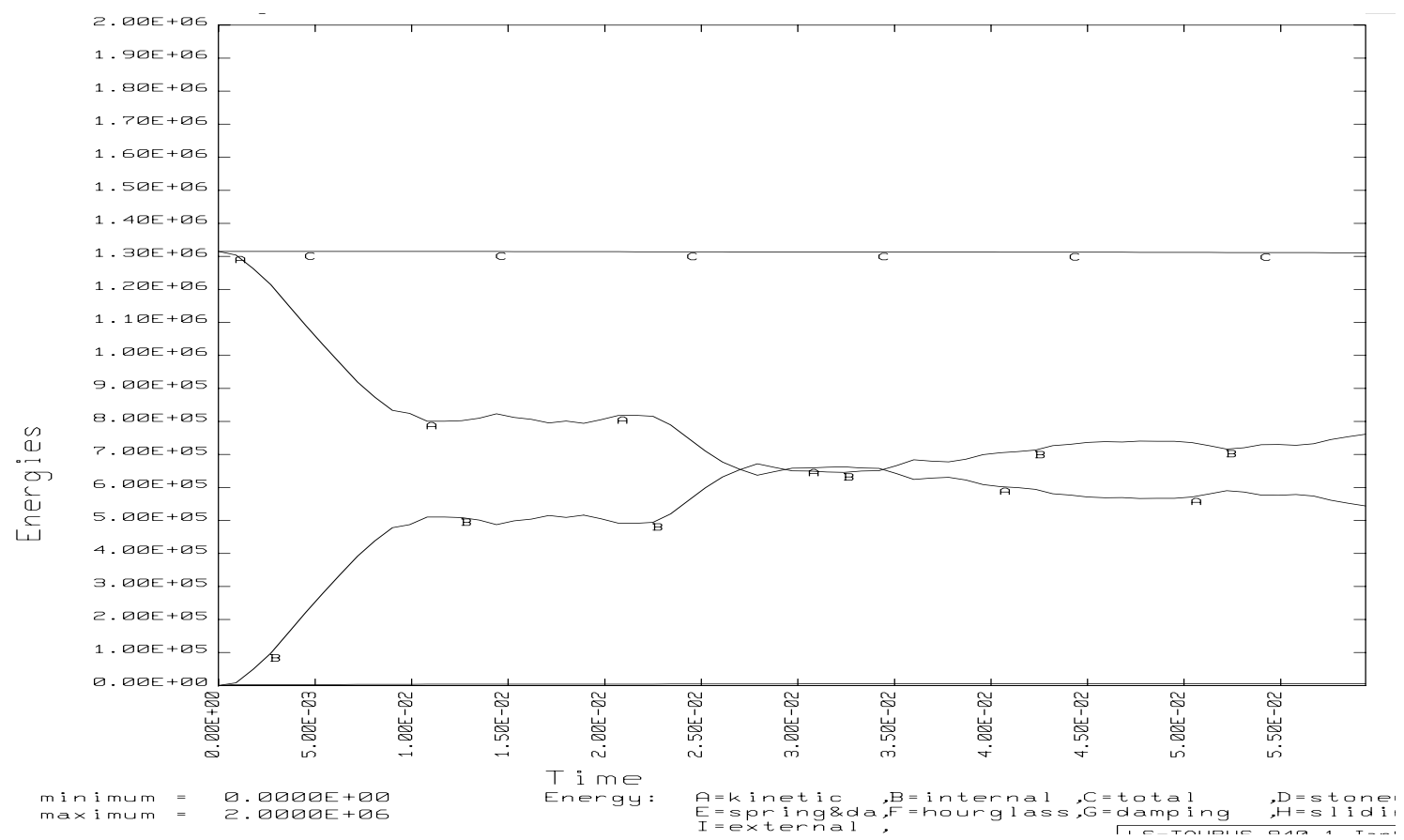

Fig 5.114 Internal, kinetic and total energies for an offset frontal impact with Hughes-Liu SR thin shells. 


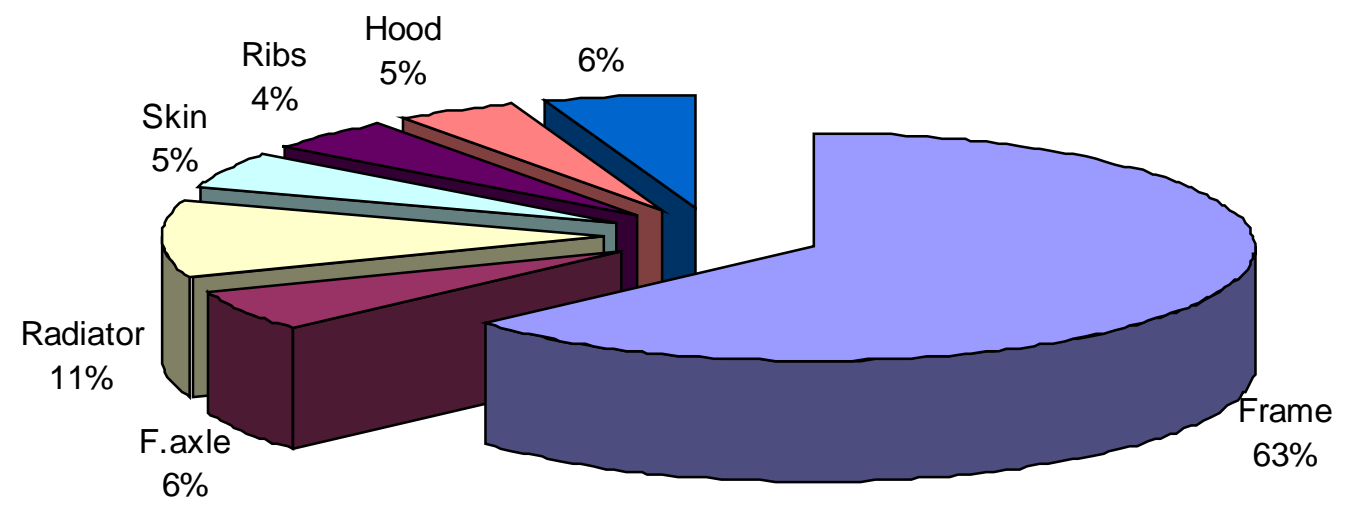

Fig 5.115 Total internal energy distribution at time $=\mathbf{4 5}$ msec of the bus subjected to an offset frontal impact with Hughes-Liu SR thin shells. 


\section{CHAPTER 6 -CONCLUSIONS AND RECOMMENDATIONS}

\subsection{CONCLUSions}

The finite element model of the bus was developed using I-DEAS Master Series software. Quadrilateral thin shell elements were used for sheet metal components and solid elements were used for the solid blocks to mesh the parts of the bus. The explicit time integration method was used to solve the model with eight-point integration for solid elements and single-point integration for thin shell elements. The single-point integration gives rise to the zero energy modes which produce the hourglass mode shapes. Since eight-point integration was used for solid elements, hourglassing was completely eliminated in all the solid elements and the hourglassing energy present in the energy balance is only from the thin shell elements. So a higher order integration for the thin shell elements was used which increased the solution time by five times the single-point integration. To avoid the hourglass energy for the thin shell elements, Hughes-Liu elements with Selective-Reduced integration were used. These elements are integrated using 2 X 2 Gaussian quadrature in the plane, and these elements are extremely useful in places where hourglassing is a problem.

Contact type 3 in LS-DYNA3D was defined between the surfaces of the front end components of the bus, which gave rise to hourglassing energy. This resulted in the continuous increase of the total energy in the system untill the end of the analysis. The contact type 3 was changed to contact type 4 and contact type 5 in LS-DYNA3D. Contact type 4 creates contact between the folding surfaces of the same part or material. Contact type 5 creates contact between the nodes of a slave surface to the element face on the 
master surface. These type of contacts did not create hour glassing energy and the total energy of the system remained constant. Figure 6.1 shows the instability in the total energy of the system when hourglassing was high for the elements in the front end of the bus.

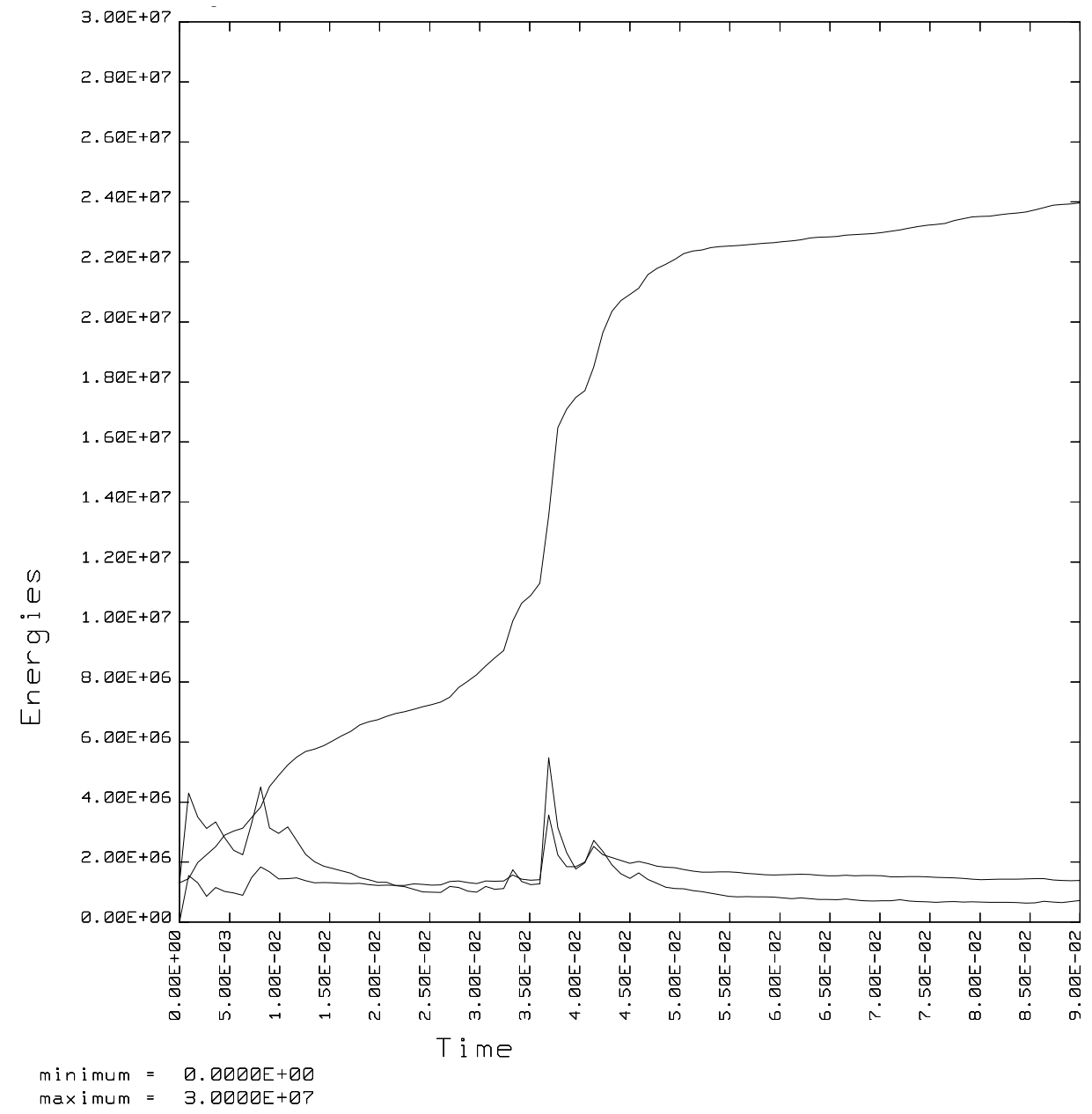

Fig 6.1 Instability in the total energy of the system with high hour glassing.

Different types of simulations were performed with the finite element model in LSDYNA3D to analyze full frontal and offset frontal crash scenarios. The full frontal impact consisted of the bus hitting an infinitely rigid wall at a velocity of $56 \mathrm{~km} / \mathrm{hr}$. Since the velocity of the bus decreases, the kinetic energy of the bus decreases and it is 
converted into material internal energy. The general characteristics like the displacement, velocity, and acceleration of the parts provide an indication of the deformation undergone by the parts of the bus.

The frame absorbs the maximum amount of kinetic energy converted as material internal energy for a full frontal impact. The occupant area in the bus lies inside the rib structure and it is covered by the inner skin and the outer skin. In a full frontal impact, the occupant area remains unaffected and the deformation of the front end components are only up to the fire wall of the bus. The deformation in the fire wall causing an intrusion into the driver's compartment is small. There is a small deformation in the body skin of the bus and the two layers of skin do not intrude but instead bulge outwards, which is not harmful to the occupants. The vehicle rotates about the $\mathrm{X}$ axis, which is called the pitching of the vehicle. This may cause the occupants in the bus to be thrown out of their seats and hit the back of the front seat, which might result in serious injuries. When the analysis was run with Hughes-Liu SR thin shell elements, the body structure pitching was less, and the pitching of the frame was high.

The offset frontal impact consisted of only one side of the bus hitting an infinitely rigid wall at a velocity of $56 \mathrm{~km} / \mathrm{hr}$. Only the right half (door way) of the bus hits the rigid wall and decelerates. The kinetic energy of the bus is converted into material internal energy since the kinetic energy decreases. The frame absorbs the maximum energy in this case and it buckles after the second engine mounting cross member. This deformation in the frame absorbs the maximum energy and protects the impact force from being transmitted to the occupant area. The front axle rotates and the right wheel hits the fire wall. This might cause a deformation in the body structure near the doorway 
of the bus. The energy absorbed by the rib structure that protects the occupant area is small compared to the front end components, which shows that the deformation is less in the bus body and there is no intrusion of any components of the bus into the occupant area.

If the impact had been simulated for the driver side half of the bus, the left wheel may have intruded into the driver occupant area after the fire wall. The vehicle pitches in this case also, which shows that the occupants could be harmed due to the pitching of the vehicle and the occupants being thrown out of their seats. In this case when the analysis was run with Hughes-Liu SR thin shell elements, the pitching was high compared to the analysis without Hughes-Liu SR thin shell elements.

Two simulation runs were made for the full frontal and the offset frontal crash scenarios, one without Hughes-Liu SR thin shell elements and the other with Hughes-Liu SR thin shell elements. The analysis without Hughes-Liu SR thin shell and with the default LS-DYNA3D shell element formulation, had hourglassing energy and the sum of the internal and the kinetic energies was not equal to the total energies. The second case, with the Hughes-Liu SR thin shell element formulation, had zero hourglassing energy and the sum of the internal and the kinetic energies was equal to the total energy. The kinetic energy decreases from the beginning of the solution and the internal energy increases and both the curves meet at exactly half the total energy.

The behavior of the vehicles in both cases was different: however, the frame remained as the principal energy absorbing part in the impact for the full frontal and the offset frontal impact with and without Hughes-Liu SR thin shell elements. So higher 
order integration for the thin shell elements with Hughes-Liu thin shell formulation is recommended in this case. The frame plays an important role in the full frontal and the offset frontal impact as it buckles after the second engine mounting cross member and absorbs a major chunk of the kinetic energy as internal energy. Since it is evident from the results that the frame plays an important role in the full frontal and the offset frontal impacts, modeling the frame is recommended for the frontal crash impact scenarios. The time taken for the solution with Hughes-Liu SR thin shells is at least five times the time taken for the analysis without Hughes-Liu SR thin shell elements.

This thesis demonstrates the ability of numerical simulation to simulate two different scenarios of impact. This project studies the deformation behavior of the vehicle and its effect on the occupants of the bus. The deformation on the bus body structure would help to study the intrusion of the bus components in the occupant area in the event of a crash. The model is a design tool that can be used to study the effects of changing component design. These changes could include lowering the center of gravity and/or changing component materials (steel to aluminum). The model could also be used to examine the effects of changing the frame rail design to prevent pitching of the vehicle in a frontal collision. The validation and the accuracy of the results obtained from the simulation have to be checked. The National Highway Traffic Safety Administration does not have adequate data that could correspond to the crashworthiness of a Type $\mathrm{C}$ school bus. This emphasizes the need for tests to be performed on a school bus to study and compare the behavior of the school bus against the simulation results. The changes that can be incorporated in the model is the reduction in the number of elements for the tire since the tire takes more number of elements and hence more solution time. Also changes 
could be incorporated by accounting for the changes in material properties during the steel forming process as done by Kaufman and Gaines ${ }^{22}$.

The side impact and rear impact could be simulated by modeling a rigid wall to the side and the rear of the bus respectively. Since the mesh density for the all the components in the bus is close enough to study the deformation shapes, the same finite element model can be used without any changes. The frame plays an important role in absorbing as much energy as possible during the crash event, which emphasizes the need of modeling the frame for frontal impacts. The designer could use the output from this work for an improvement in design of the school buses. 


\section{CHAPTER - 8 REFERENCES}

${ }^{1}$ Frank F. Monasa and William Mcguire, "Collapse Analysis of Bus Body Structures using Interactive Computer Graphics," SAE Paper 860824, Society of Automotive Engineers, Warrendale, U.S.A 1986.

${ }^{2}$ Michael Y. Sheh, John D. Reid, Stephen M. Lesh and Wichai Cheva, "Vehicle Crashworthiness Analysis Using Numerical Methods and Experiments," SAE Paper 921075, Society of Automotive Engineers, Warrendale, U.S.A 1992.

${ }^{3}$ Helcio Onusic, Jose` Augusto P Campos and Paulo Sergio P dos Santos, "Considerations Concerning Vehicle Collisions Through Simplified Calculation of the Impact Forces," SAE Paper 962324, Society of Automotive Engineers, Warrendale, U.S.A 1996.

${ }^{4}$ M.Igarashi, "1989 Suzuki Sidekick/Geo Tracker Body Structure Analysis,” SAE Paper 892536, Society of Automotive Engineers, Warrendale, U.S.A 1989.

${ }^{5}$ Donald A. Vander Lugt, Roger J. Chen and Ashok S. Deshpande, "Passenger Car Frontal Barrier Simulation Using Nonlinear Finite Element Methods," SAE Paper 871958, Society of Automotive Engineers, Warrendale, U.S.A 1987

${ }^{6}$ Ted Belytschko, “On Computational Methods for Crashworthiness,” SAE Paper 880893, Society of Automotive Engineers, Warrendale, U.S.A 1988. 
${ }^{7}$ Balike Mahesh, Rakheja Subash and Hon Suong Yan, “ Crashworthiness Enhancement in a Car-Truck Collision Using Energy Dissipative Under-ride Guard," SAE Paper 962211, Society of Automotive Engineers, Warrendale, U.S.A 1996.

${ }^{8}$ Michael A. Piatak, Michael Y. Sheh, Song L. Young, and James Y. Chen, "Using Nonlinear Finite Element Method in Convertible Crashworthiness Design," SAE Paper 951077, Society of Automotive Engineers, Warrendale, U.S.A 1995.

${ }^{9}$ I-DEAS STUDENT GUIDE, Structural Dynamics and Research Corp., 1997.

${ }^{10}$ Victor H. Mucino, "Review of Crashworthiness Technology," Center For Industrial Research and Applications, WVU.

${ }^{11}$ NHTSA, "List of School Bus Related Federal Motor Vehicle Safety Standards," School Bus Safety Assurance Program, 1997.

${ }^{12}$ Toru Aida, "IDEADYN," Center for Industrial Research Applications (CIRA), Mechanical and Aerospace Engineering Department, West Virginia University.

${ }^{13}$ Anand Ravipati, “A Vehicle Crashworthiness Simulation Model,” Master of Science Thesis submitted to the College of Engineering, West Virginia University, 1994. 
${ }^{14}$ Eric Saunders, "School Bus Children-Seat Interaction In Frontal Collisions: An Articulated Seat," Master of Science Thesis submitted to the college of Engineering and Mineral Resources, West Virginia University, 1997.

${ }^{15}$ SDRC,'I-DEAS Master Series 4.0," Structural Dynamics Research Corporation, Milford, Ohio.

${ }^{16}$ Livermore Software Technology Corporation, "LS-TAURUS".

${ }^{17}$ Livermore Software Technology Corporation, "NIKE3D”.

${ }^{18}$ National Technical Information Service, Springfield, VA 22161.

${ }^{19}$ W.Riley Garrot, Mark A. Flick and Elizabeth N. Mazzae, "Hardware Evaluation of Heavy Truck Side and Rear Object Detection Systems," SAE Paper 951010, Society of Automotive Engineers, Warrendale, U.S.A 1995.

${ }^{20}$ George Rechnitzer, Gray Scott and Noel Murray, "The Reduction of Injuries to Car Occupants in Rear End Impacts with Heavy Vehicles," SAE Paper 933123, Society of Automotive Engineers, Warrendale, U.S.A 1993. 
${ }^{21}$ Yukio Shiosaka, "Research on the Evacuation Readiness of Bus Crews and Passengers - Investigation of the effect of a New Type of Exit," SAE Paper 962210, Society of Automotive Engineers, Warrendale, U.S.A 1996.

${ }^{22}$ Mark Kaufman, David Gaines, Ken Kundrick and Sheng-Dong Liu, "Integration of Chassis Frame Forming Analysis into Performance Models to More Accurately Evaluate Crashworthiness," SAE Paper 980551, Society of Automotive Engineers, Warrendale, U.S.A 1998.

${ }^{23}$ C.Brian Tanner, H.Fred Chen, John F. Weichel, Douglas R. Brown and Dennis A. Guenther, "Vehicle and Occupant Response in Heavy truck to Car Low-Speed rear Impact," SAE Paper 970120, Society of Automotive Engineers, Warrendale, U.S.A 1997.

${ }^{24}$ Livermore Software Technology Corporation, "LS-DYNA3D Users Manual", Version 940.1 .

${ }^{25}$ Sun X, Toth R, Wiedmann T, "Development of the GM 5.7L CNG Bi-Fuel Pickup Trucks," SAE Paper 980817, Society of Automotive Engineers, Warrendale, U.S.A 1998.

\footnotetext{
${ }^{26}$ National Highway Traffic Safety Administration, "Traffic Safety Facts 1993," US Department of Transportation.
} 
${ }^{27}$ National Technical Information Service, "Highway Accident report, School Bus - Loss of Control and Collision With Guard Rail and Sign Pillar, U.S. Highway 70 Near Luca and Hunt Road, St.Louis County, Missouri,” April 14,1987.

${ }^{28}$ National Transportation Safety Board, “Crashworthiness of Small Post standard School Buses," October 11, 1989.

${ }^{29}$ National Technical Information Service, "Highway Accident report, Collision of Small School Bus and Tractor-Semi Trailer," November 29, 1994.

${ }^{30}$ National Technical Information Service, "Highway Accident report, Collision of Northeast Illinois Regional Commuter Railroad Corporation (METRA) Train and Transportation Joint Agreement School District 47/155 School Bus at Railroad/Highway Grade Crossing in Fox River Grove, Illinois,” October 291996.

${ }^{31}$ Livermore Software Technology Corporation, "LS-DYNA3D Theory Manual”, Version 940.1 


\section{APPENDIX A - FMVSS FOR SCHOOL BUS}

Federal Motor Vehicle Safety Standard No.105, "Hydraulic Brake System”.

Federal Motor Vehicle Safety Standard No.111, "Rearview Mirrors".

Federal Motor Vehicle Safety Standard No.115, "Vehicle Identification Number".

Federal Motor Vehicle Safety Standard No.120, "Tire Selection and Rims for vehicles Other than Passenger Cars".

Federal Motor Vehicle Safety Standard No.121, "Air Brake Systems”.

Federal Motor Vehicle Safety Standard No.131, "School Bus Pedestrian Safety Devices".

Federal Motor Vehicle Safety Standard No.209, "Safety Belt Assemblies".

Federal Motor Vehicle Safety Standard No.210, "Safety Belt Assembly Anchorages".

Federal Motor Vehicle Safety Standard No.217, "Bus Window Retention and Release".

Federal Motor Vehicle Safety Standard No.221, "School Bus Body Joint Strength".

Federal Motor Vehicle Safety Standard No.222, "School Bus Passenger Seating”.

Federal Motor Vehicle Safety Standard No.301, "Fuel System Integrity".

Federal Motor Vehicle Safety Standard No.304, “Compressed natural Gas Fuel Container Integrity". 


\section{APPENDIX B - CONDENSED LS-DYNA3D INPUT FILE}

* Analysis input data for LS-DYNA3D 92

* Translated from I-DEAS universal file buside.unv

* written by I-DEAS Master Series 4: Simulation

* vis IDEADYN Revision 9.5 .

* Title

VC LARGE

/extra1/rajan/crashbus/crashbus.mf1 92 large

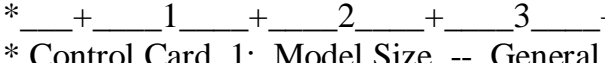

* NMMAT/ NUMNP/ NUMELH/ NUMELB/ NUMELS/ NUMELT/ NUSRMT $\begin{array}{lllllll}18 & 30038 & 7481 & 0 & 15224 & 0 & 0\end{array}$

$*$

* Control Card 2: Model Size -- Boundary Conditions

*NSPC/COOR/VEAC/NRBS/ RC/ RCF/ JOY

$\begin{array}{llllllll}0 & 0 & 0 & 0 & 0 & 0 & 0 & 0\end{array}$

*

* Control Card 3: Model Size -- Loading

*LDCV/CNLD/ PLC/ GBL/ BPC/DTPT/ELMD/ NDP

$\begin{array}{llllllll}1 & 64 & 0 & 0 & 0 & 0 & 0 & 0\end{array}$

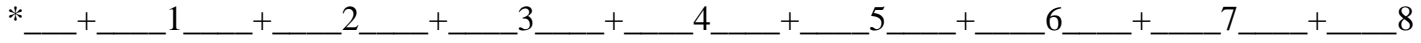

* Control Card 4: Model Size -- Constraints and Contact

* RWL/ SI/ BLK/TBSL/TNWF/TNPF/NNCC/NCEQ/NOSL

$\begin{array}{lllllllll}0 & 1 & 0 & 0 & 0 & 0 & 0 & 0 & 0\end{array}$

*

* Control Card 5: Model Size -- Rigid Body Parameters

* RBS/ RBC/ JTD/EXTR/ RBI

$\begin{array}{llllllll}0 & 0 & 0 & 0 & 0 & 0 & 0\end{array}$

*

* Control Card 6: Model Size -- Discrete Elements and Seat Belts

*MTDF/CORD/ ELD/ MAS/ SBM/ SBE/SBSR/SBRT/SBSE/SBPT/ ACC

$\begin{array}{llllllllllll}0 & 0 & 0 & 0 & 0 & 0 & 0 & 0 & 0 & 0 & 0\end{array}$

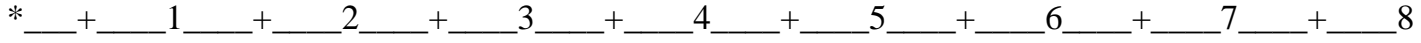

* Control Card 7: Model Size -- Output Control

* CSD/NOFG/ IFS/RWSG

$\begin{array}{llll}0 & 0 & 0 & 0\end{array}$

$*$

* Control Card 8: Model Size -- Termination

* ENDTIM /TERM.CYCL/ TSMIN /\%DE-RATIO

8.000E-02 $00.000 \mathrm{E}+00 \quad 00.000 \mathrm{E}+00$

$*$

* Control Card 9: Computation Options -- Time Step Size Control

* DT2OLD / TSSFAC / ISDO / TSLIMT/ DT2MS / LCTM

$0.000 \mathrm{E}+009.000 \mathrm{E}-01 \quad 00.000 \mathrm{E}+000.000 \mathrm{E}+00 \quad 0 \quad 0$

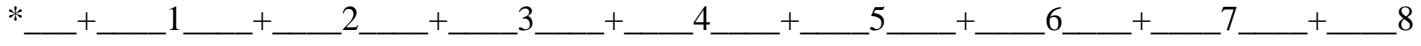

* Control Card 10: Computation Options -- Body Forces

*X-AC/Y-AC/Z-AC/XROT/YROT/ZROT

$\begin{array}{llllll}0 & 0 & 0 & 0 & 0 & 0\end{array}$

*

* Control Card 11: Computation Options -- Input Control

*INIT/NSRT/IARB/ NIF/ CNV/BROD

$\begin{array}{lllll}1 & 0 & 0 \mathrm{E} 20.0 & 0 & 0\end{array}$ 
$*$

* Control Card 12: Computation Options -- Beams and Shells

* WRPANG /MITR/TRST/RNXX/SUPD/BELY/UBIR/PUBR/USIR/PUSR

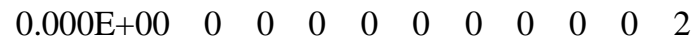

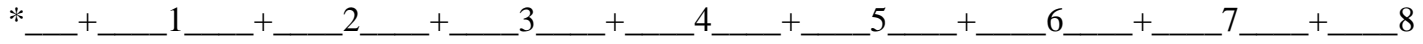

* Control Card 13: Computation Options -- Material Related Input

* IHQ/QH-DEFLT./ IBQ/Q1 (QUAD)/Q2 (LIN.)/RDFL/RDMS/TEMP

1 1.000E-01 1 1.500E+00 6.000E-02 000000

$*$

* Control Card 14: Computation Options -- Damping / Dynamic Relaxation

*LCDP/ VALDMP/DRFL/CYCK/ DRTOL / DRFCTR / DRTERM / TSSFDR /DRAL/ EDTTL $00.000 \mathrm{E}+00 \quad 02501.000 \mathrm{E}-03$ 9.950E-01 0.000E+00 0.000E+00 $00.000 \mathrm{E}+00$

$*$

* Control Card 15: Computation Options -- Contact

* SLSFAC / RWPNAL /INIC/THIK/ PSV/STCC/INIR/SCIC/SCIF/IGEO/ BCS/INTS $\begin{array}{llllllllllll}0.000 \mathrm{E}+00 & 0.000 \mathrm{E}+00 & 0 & 0 & 0 & 0 & 0 & 0 & 0 & 10 & 0 & 0.000 \mathrm{E}+00\end{array}$

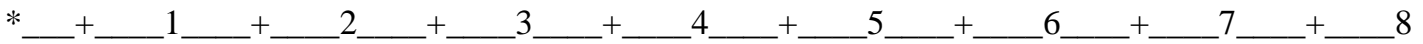

* Control Card 16: Computation Options -- Parallel and Subcycling

*NCPU/SORT/SUBS/

100

$*$

* Control Card 17: Computation Options -- Coupling

* UNLEN / UNTIME / UNFORCE /MREP/

$0.000 \mathrm{E}+000.000 \mathrm{E}+00 \quad 0.000 \mathrm{E}+00 \quad 0 \quad 0000.000 \mathrm{E}+00 \quad 0$

* Control Card 18: Computation Options -- Output Control

*POPT/TSPF/EDIT/RDEC/CBRF/ECHO/DEBG/RFUP/ACCA/OPIF/

$\begin{array}{llllllllll}0 & 0 & 0 & 0 & 0 & 0 & 0 & 0 & 0 & 0.000 \mathrm{E}+00\end{array}$

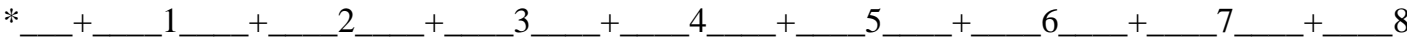

* Control Card 19: Computation Options -- Output Energy

*HGEC/NRWO/SIED/RDED

$2 \quad 2 \quad 2 \quad 2$

$*$

* Control Card 20: Computation Options -- TAURUS Database Control I

*STATEDUMP/ IFF-DUMP/THDT-DUMP/NDTH/NSTH/NSTB/NSTS/NSTT

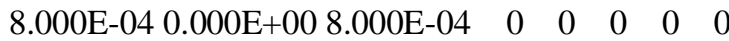

$*$

* Control Card 21: Computation Options -- TAURUS Database Control II

*DREL/NIPH/NIPS/SINT/STRN/STEN/EFPS/SRES/IETH/PSEP/

$\begin{array}{lllllllllll}0 & 0 & 0 & 3 & 1 & 1 & 1 & 1 & 1 & 0 & 0\end{array}$

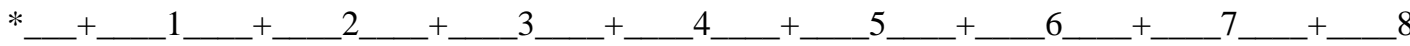

* Control Card 22: Computation Options -- ASCII Output Control I

* SECFORC / RWFORC / NODOUT / ELOUT / GLSTAT / DEFORC / MATSUM / NCFORC 8.000E-04 8.000E-04 8.000E-04 8.000E-04 8.000E-04 8.000E-04 8.000E-04 8.000E-04

$*$

* Control Card 23: Computation Options -- ASCII Output Control II

* RCFORC / DEFGEO / SPCFORC / SWFORC / ABSTAT / AVSFLT / NODFOR / BNDOUT

$0.000 \mathrm{E}+000.000 \mathrm{E}+000.000 \mathrm{E}+00 \quad 0.000 \mathrm{E}+000.000 \mathrm{E}+000.000 \mathrm{E}+000.000 \mathrm{E}+000.000 \mathrm{E}+00$

*

* Control Card 24: Computation Options -- ASCII Output Control III

* RBDOUT / GCEOUT / MPGS / MOVIE / SLEOUT / SBTOUT /

8.000E-04 0.000E+00 0.000E+00 0.000E+00 0.000E+00 0.000E+00 0.000E+00

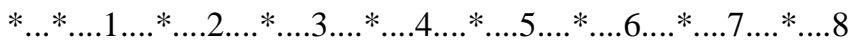

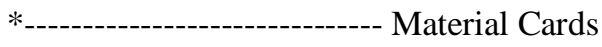

*** MATERIAL: 1 
$\begin{array}{lllllllllll}1 & 17798.0000 & 0 & 0 & 0.0000000 & 0 & 0.0000000 & 0.0000000 & 2 & 0 & 6\end{array}$

material type \#1 (Linear-Elastic Thin shell)

$2.0700+110.00000000 .00000000 .00000000 .00000000 .00000000 .00000000 .0000000$ 0.33000000 .00000000 .00000000 .00000000 .00000000 .00000000 .00000000 .0000000 0.00000000 .00000000 .00000000 .00000000 .00000000 .00000000 .00000000 .0000000 0.00000000 .00000000 .00000000 .00000000 .00000000 .00000000 .00000000 .0000000 0.00000000 .00000000 .00000000 .00000000 .00000000 .00000000 .00000000 .0000000 0.00000000 .00000000 .00000000 .00000000 .00000000 .00000000 .00000000 .0000000 section properties

$\begin{array}{llll}0.0000000 & 0 & 0 & 0\end{array}$

6.00000-3 6.00000-3 6.00000-3 6.00000-3 0.0000000

*** MATERIAL: 2

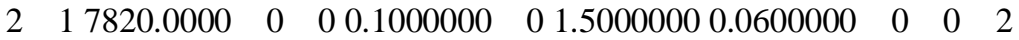

material type \#1 (Linear Elastic - Brick)

$2.0700+110.00000000 .00000000 .00000000 .00000000 .00000000 .00000000 .0000000$ 0.33000000 .00000000 .00000000 .00000000 .00000000 .00000000 .00000000 .0000000 0.00000000 .00000000 .00000000 .00000000 .00000000 .00000000 .00000000 .0000000 0.00000000 .00000000 .00000000 .00000000 .00000000 .00000000 .00000000 .0000000 0.00000000 .00000000 .00000000 .00000000 .00000000 .00000000 .00000000 .0000000 0.00000000 .00000000 .00000000 .00000000 .00000000 .00000000 .00000000 .0000000 *** MATERIAL: 3

$\begin{array}{llllllllllll}3 & 1782.00000 & 0 & 0 & 0.1000000 & 0 & 1.5000000 & 0.0600000 & 0 & 0 & 2\end{array}$

material type \#1 (Linear Elastic - Brick)

$2.0700+110.00000000 .00000000 .00000000 .00000000 .00000000 .00000000 .0000000$ 0.33000000 .00000000 .00000000 .00000000 .00000000 .00000000 .00000000 .0000000 0.00000000 .00000000 .00000000 .00000000 .00000000 .00000000 .00000000 .0000000 0.00000000 .00000000 .00000000 .00000000 .00000000 .00000000 .00000000 .0000000 0.00000000 .00000000 .00000000 .00000000 .00000000 .00000000 .00000000 .0000000 0.00000000 .00000000 .00000000 .00000000 .00000000 .00000000 .00000000 .0000000 *** MATERIAL: 4

$\begin{array}{llllllllllll}4 & 17798.0000 & 0 & 0 & 0.0000000 & 0 & 0.0000000 & 0.0000000 & 2 & 0 & 6\end{array}$ material type \#1 (Linear-Elastic Thin shell)

$2.0700+110.00000000 .00000000 .00000000 .00000000 .00000000 .00000000 .0000000$ 0.33000000 .00000000 .00000000 .00000000 .00000000 .00000000 .00000000 .0000000 0.00000000 .00000000 .00000000 .00000000 .00000000 .00000000 .00000000 .0000000 0.00000000 .00000000 .00000000 .00000000 .00000000 .00000000 .00000000 .0000000 0.00000000 .00000000 .00000000 .00000000 .00000000 .00000000 .00000000 .0000000 0.00000000 .00000000 .00000000 .00000000 .00000000 .00000000 .00000000 .0000000 section properties

$\begin{array}{llll}0.0000000 & 0 & 0 & 0\end{array}$

6.00000-3 6.00000-3 6.00000-3 6.00000-3 0.0000000

*** MATERIAL: 5

$\begin{array}{llllllllllll}5 & 17820.0000 & 0 & 0 & 0.1000000 & 0 & 1.5000000 & 0.0600000 & 0 & 0 & 6\end{array}$

material type \#1 (Linear Elastic - Thin shell)

$2.0700+110.00000000 .00000000 .00000000 .00000000 .00000000 .00000000 .0000000$ 0.33000000 .00000000 .00000000 .00000000 .00000000 .00000000 .00000000 .0000000 0.00000000 .00000000 .00000000 .00000000 .00000000 .00000000 .00000000 .0000000 0.00000000 .00000000 .00000000 .00000000 .00000000 .00000000 .00000000 .0000000 0.00000000 .00000000 .00000000 .00000000 .00000000 .00000000 .00000000 .0000000 0.00000000 .00000000 .00000000 .00000000 .00000000 .00000000 .00000000 .0000000 *** MATERIAL: 6

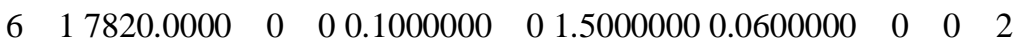

material type \#1 (Linear Elastic - Brick)

$2.0700+110.00000000 .00000000 .00000000 .00000000 .00000000 .00000000 .0000000$ 0.33000000 .00000000 .00000000 .00000000 .00000000 .00000000 .00000000 .0000000 0.00000000 .00000000 .00000000 .00000000 .00000000 .00000000 .00000000 .0000000 
0.00000000 .00000000 .00000000 .00000000 .00000000 .00000000 .00000000 .0000000 0.00000000 .00000000 .00000000 .00000000 .00000000 .00000000 .00000000 .0000000 0.00000000 .00000000 .00000000 .00000000 .00000000 .00000000 .00000000 .0000000 *** MATERIAL: 7

$\begin{array}{lllllllllll}7 & 17798.0000 & 0 & 0 & 0.0000000 & 0 & 0.0000000 & 0.0000000 & 2 & 0 & 6\end{array}$ material type \#1 (Linear-Elastic Thin shell)

$2.0700+110.00000000 .00000000 .00000000 .00000000 .00000000 .00000000 .0000000$ 0.33000000 .00000000 .00000000 .00000000 .00000000 .00000000 .00000000 .0000000 0.00000000 .00000000 .00000000 .00000000 .00000000 .00000000 .00000000 .0000000 0.00000000 .00000000 .00000000 .00000000 .00000000 .00000000 .00000000 .0000000 0.00000000 .00000000 .00000000 .00000000 .00000000 .00000000 .00000000 .0000000 0.00000000 .00000000 .00000000 .00000000 .00000000 .00000000 .00000000 .0000000 section properties

$\begin{array}{llll}0.0000000 & 0 & 0 & 0\end{array}$

2.00000-3 2.00000-3 2.00000-3 2.00000-3 0.0000000

*** MATERIAL: 8

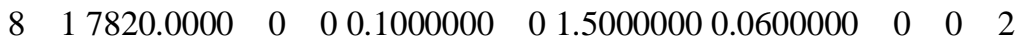

material type \#1 (Linear Elastic - Brick)

$2.0700+110.00000000 .00000000 .00000000 .00000000 .00000000 .00000000 .0000000$

0.33000000 .00000000 .00000000 .00000000 .00000000 .00000000 .00000000 .0000000

0.00000000 .00000000 .00000000 .00000000 .00000000 .00000000 .00000000 .0000000 0.00000000 .00000000 .00000000 .00000000 .00000000 .00000000 .00000000 .0000000 0.00000000 .00000000 .00000000 .00000000 .00000000 .00000000 .00000000 .0000000 0.00000000 .00000000 .00000000 .00000000 .00000000 .00000000 .00000000 .0000000 *** MATERIAL: 9

$\begin{array}{llllllllllll}9 & 17798.0000 & 0 & 0 & 0.0000000 & 0 & 0.0000000 & 0.0000000 & 2 & 0 & 6\end{array}$ material type \#1 (Linear-Elastic Thin shell)

$2.0700+110.00000000 .00000000 .00000000 .00000000 .00000000 .00000000 .0000000$ 0.33000000 .00000000 .00000000 .00000000 .00000000 .00000000 .00000000 .0000000 0.00000000 .00000000 .00000000 .00000000 .00000000 .00000000 .00000000 .0000000 0.00000000 .00000000 .00000000 .00000000 .00000000 .00000000 .00000000 .0000000 0.00000000 .00000000 .00000000 .00000000 .00000000 .00000000 .00000000 .0000000 0.00000000 .00000000 .00000000 .00000000 .00000000 .00000000 .00000000 .0000000 section properties

$\begin{array}{llll}0.0000000 & 0 & 0 & 0\end{array}$

1.00000-3 1.00000-3 1.00000-3 1.00000-3 0.0000000

*** MATERIAL: 10

$\begin{array}{llllllllllll}10 & 17820.0000 & 0 & 0 & 0.1000000 & 0 & 1.5000000 & 0.0600000 & 0 & 0 & 2\end{array}$

material type \#1 (Linear Elastic - Brick)

$2.0700+110.00000000 .00000000 .00000000 .00000000 .00000000 .00000000 .0000000$

0.33000000 .00000000 .00000000 .00000000 .00000000 .00000000 .00000000 .0000000 0.00000000 .00000000 .00000000 .00000000 .00000000 .00000000 .00000000 .0000000 0.00000000 .00000000 .00000000 .00000000 .00000000 .00000000 .00000000 .0000000 0.00000000 .00000000 .00000000 .00000000 .00000000 .00000000 .00000000 .0000000 0.00000000 .00000000 .00000000 .00000000 .00000000 .00000000 .00000000 .0000000 *** MATERIAL: 11

$\begin{array}{llllllllllll}11 & 1 & 7820.0000 & 0 & 0 & 0.1000000 & 0 & 1.5000000 & 0.0600000 & 2 & 0 & 6\end{array}$ material type \#1 (Linear Elastic - Thin shell)

$2.0700+110.00000000 .00000000 .00000000 .00000000 .00000000 .00000000 .0000000$ 0.33000000 .00000000 .00000000 .00000000 .00000000 .00000000 .00000000 .0000000 0.00000000 .00000000 .00000000 .00000000 .00000000 .00000000 .00000000 .0000000 0.00000000 .00000000 .00000000 .00000000 .00000000 .00000000 .00000000 .0000000 0.00000000 .00000000 .00000000 .00000000 .00000000 .00000000 .00000000 .0000000 0.00000000 .00000000 .00000000 .00000000 .00000000 .00000000 .00000000 .0000000 section properties

$0.0000000 \quad 0 \quad 0 \quad 0$ 
1.00000-3 1.00000-3 1.00000-3 1.00000-3 0.0000000

*** MATERIAL: 12

$\begin{array}{lllllllllllll}12 & 1 & 7820.0000 & 0 & 0 & 0.1000000 & 0 & 1.5000000 & 0.0600000 & 0 & 0 & 2\end{array}$

material type \#1 (Linear Elastic - Brick)

$2.0700+110.00000000 .00000000 .00000000 .00000000 .00000000 .00000000 .0000000$

0.33000000 .00000000 .00000000 .00000000 .00000000 .00000000 .00000000 .0000000

0.00000000 .00000000 .00000000 .00000000 .00000000 .00000000 .00000000 .0000000

0.00000000 .00000000 .00000000 .00000000 .00000000 .00000000 .00000000 .0000000

0.00000000 .00000000 .00000000 .00000000 .00000000 .00000000 .00000000 .0000000

0.00000000 .00000000 .00000000 .00000000 .00000000 .00000000 .00000000 .0000000

*** MATERIAL: 13

$\begin{array}{llllllllllll}13 & 1 & 7820.0000 & 0 & 0 & 0.1000000 & 0 & 1.5000000 & 0.0600000 & 0 & 0 & 2\end{array}$

material type \#1 (Linear Elastic - Brick)

$2.0700+110.00000000 .00000000 .00000000 .00000000 .00000000 .00000000 .0000000$

0.33000000 .00000000 .00000000 .00000000 .00000000 .00000000 .00000000 .0000000

0.00000000 .00000000 .00000000 .00000000 .00000000 .00000000 .00000000 .0000000

0.00000000 .00000000 .00000000 .00000000 .00000000 .00000000 .00000000 .0000000

0.00000000 .00000000 .00000000 .00000000 .00000000 .00000000 .00000000 .0000000

0.00000000 .00000000 .00000000 .00000000 .00000000 .00000000 .00000000 .0000000

*** MATERIAL: 14

$14 \quad 17798.0000 \quad 0 \quad 00.0000000 \quad 00.00000000 .0000000 \quad 2 \quad 0 \quad 6$

material type \#1 (Linear-Elastic Thin shell)

$2.0700+110.00000000 .00000000 .00000000 .00000000 .00000000 .00000000 .0000000$ 0.33000000 .00000000 .00000000 .00000000 .00000000 .00000000 .00000000 .0000000 0.00000000 .00000000 .00000000 .00000000 .00000000 .00000000 .00000000 .0000000 0.00000000 .00000000 .00000000 .00000000 .00000000 .00000000 .00000000 .0000000 0.00000000 .00000000 .00000000 .00000000 .00000000 .00000000 .00000000 .0000000 0.00000000 .00000000 .00000000 .00000000 .00000000 .00000000 .00000000 .0000000 section properties

$\begin{array}{llll}0.0000000 & 0 & 0 & 0\end{array}$

2.00000-3 2.00000-3 2.00000-3 2.00000-3 0.0000000

*** MATERIAL: 15

$\begin{array}{llllllllllll}15 & 1 & 7820.0000 & 0 & 0 & 0.1000000 & 0 & 1.5000000 & 0.0600000 & 0 & 0 & 2\end{array}$

material type \#1 (Linear Elastic - Brick)

$2.0700+110.00000000 .00000000 .00000000 .00000000 .00000000 .00000000 .0000000$ 0.33000000 .00000000 .00000000 .00000000 .00000000 .00000000 .00000000 .0000000 0.00000000 .00000000 .00000000 .00000000 .00000000 .00000000 .00000000 .0000000 0.00000000 .00000000 .00000000 .00000000 .00000000 .00000000 .00000000 .0000000 0.00000000 .00000000 .00000000 .00000000 .00000000 .00000000 .00000000 .0000000 0.00000000 .00000000 .00000000 .00000000 .00000000 .00000000 .00000000 .0000000 *** MATERIAL: 16

$\begin{array}{lllllllllll}16 & 17798.0000 & 0 & 0 & 0.0000000 & 0 & 0.0000000 & 0.0000000 & 2 & 0 & 6\end{array}$ material type \#1 (Linear-Elastic Thin shell)

$2.0700+110.00000000 .00000000 .00000000 .00000000 .00000000 .00000000 .0000000$ 0.33000000 .00000000 .00000000 .00000000 .00000000 .00000000 .00000000 .0000000 0.00000000 .00000000 .00000000 .00000000 .00000000 .00000000 .00000000 .0000000 0.00000000 .00000000 .00000000 .00000000 .00000000 .00000000 .00000000 .0000000 0.00000000 .00000000 .00000000 .00000000 .00000000 .00000000 .00000000 .0000000 0.00000000 .00000000 .00000000 .00000000 .00000000 .00000000 .00000000 .0000000 section properties

$0.0000000 \quad 0 \quad 0 \quad 0$

6.00000-3 6.00000-3 6.00000-3 6.00000-3 0.0000000

*** MATERIAL: 17

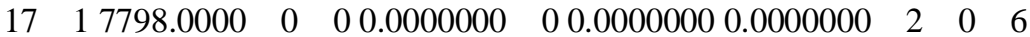

material type \#1 (Linear-Elastic Thin shell)

$2.0700+110.00000000 .00000000 .00000000 .00000000 .00000000 .00000000 .0000000$ 
0.33000000 .00000000 .00000000 .00000000 .00000000 .00000000 .00000000 .0000000 0.00000000 .00000000 .00000000 .00000000 .00000000 .00000000 .00000000 .0000000 0.00000000 .00000000 .00000000 .00000000 .00000000 .00000000 .00000000 .0000000 0.00000000 .00000000 .00000000 .00000000 .00000000 .00000000 .00000000 .0000000 0.00000000 .00000000 .00000000 .00000000 .00000000 .00000000 .00000000 .0000000 section properties

$\begin{array}{llll}0.0000000 & 0 & 0 & 0\end{array}$

2.00000-3 2.00000-3 2.00000-3 2.00000-3 0.0000000

*** MATERIAL: 18

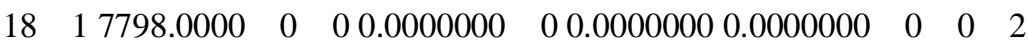

material type \#1 (Linear-Elastic Brick)

$2.0700+110.00000000 .00000000 .00000000 .00000000 .00000000 .00000000 .0000000$

0.33000000 .00000000 .00000000 .00000000 .00000000 .00000000 .00000000 .0000000

0.00000000 .00000000 .00000000 .00000000 .00000000 .00000000 .00000000 .0000000

0.00000000 .00000000 .00000000 .00000000 .00000000 .00000000 .00000000 .0000000 0.00000000 .00000000 .00000000 .00000000 .00000000 .00000000 .00000000 .0000000 0.00000000 .00000000 .00000000 .00000000 .00000000 .00000000 .00000000 .0000000

$*$

$* \ldots * \ldots 1 \ldots * \ldots . \ldots * *^{*} \ldots 3 \ldots * \ldots . \ldots 4 \ldots * \ldots 5 \ldots . \ldots * \ldots 6 \ldots * \ldots 7 \ldots * \ldots 8$

$*$

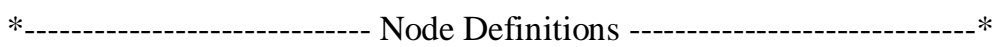

* \#/ DBC/ X-COOR / Y-COOR / Z-COOR / RBC

$1 \quad 0$ 0.0000000000000E+00 6.1199998855591E-01 0.0000000000000E+00 0

$2 \quad 0-1.5727986226699 \mathrm{E}-15$ 7.2109442949295E-01-1.5748000144958E+00 0

$3 \quad 0-2.7755575615629 \mathrm{E}-17$ 3.9098110795021E-01 0.0000000000000E+00 0

$4 \quad 0-2.2204460492503 \mathrm{E}-16-2.2859999537468 \mathrm{E}-010.0000000000000 \mathrm{E}+00 \quad 0$

$5 \quad 0-1.7157831575317 \mathrm{E}-16-3.7548467516899 \mathrm{E}-02 \quad 0.0000000000000 \mathrm{E}+00 \quad 0$

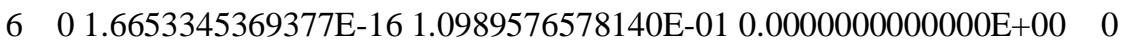

$7 \quad 0-1.0320502215742 \mathrm{E}-162.2129407525063 \mathrm{E}-010.0000000000000 \mathrm{E}+00 \quad 0$

$8 \quad 02.4980018054066 \mathrm{E}-162.7645480632782 \mathrm{E}-010.0000000000000 \mathrm{E}+00 \quad 0$

$9 \quad 0-1.4720162073859 \mathrm{E}-165.4735042154789 \mathrm{E}-020.0000000000000 \mathrm{E}+00 \quad 0$

$10 \quad 0-1.5727986226699 \mathrm{E}-15$ 4.2102333903313E-01-1.5748000144958E+00 0

$30027 \quad 0$ 1.2491999864578E+00 7.6956778764725E-01-4.6461100578308E+00 0

$30028 \quad 01.2491999864578 \mathrm{E}+00$ 7.6956778764725E-01-4.7611112594604E+00 $\quad 0$

$30029 \quad 01.2491999864578 \mathrm{E}+00$ 7.6956778764725E-01-4.8761100769043E+00 0

$30030 \quad 01.2491999864578 \mathrm{E}+00$ 7.6956778764725E-01-4.9911112785339E+00 $\quad 0$

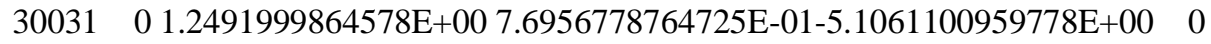

$30032 \quad 01.2491999864578 \mathrm{E}+00$ 7.6956778764725E-01-5.2211112976074E+00 $\quad 0$

$30033 \quad 01.2491999864578 \mathrm{E}+007.6956778764725 \mathrm{E}-01-5.3361101150513 \mathrm{E}+00 \quad 0$

$30034 \quad 01.2491999864578 \mathrm{E}+00$ 7.6956778764725E-01-5.4511079788208E+00 0

$30035 \quad 01.2491999864578 \mathrm{E}+00$ 7.6956778764725E-01-5.5661101341248E+00 0

$30036 \quad 01.2491999864578 \mathrm{E}+00$ 7.6956778764725E-01-5.6811113357544E+00 0

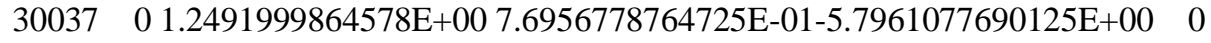

$30038 \quad 01.2491999864578 \mathrm{E}+00$ 7.6956778764725E-01-5.9111080169678E+00 0

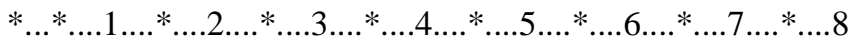

*

*--

* \#/ MAT/ N1/ N2/ N3/ N4/ N5/ N6/

$\begin{array}{llllllllll}1 & 12 & 1063 & 1062 & 1064 & 1065 & 1383 & 1382 & 1384 & 1385\end{array}$

$\begin{array}{llllllllll}2 & 12 & 1073 & 1072 & 1074 & 1075 & 1393 & 1392 & 1394 & 1395\end{array}$ 


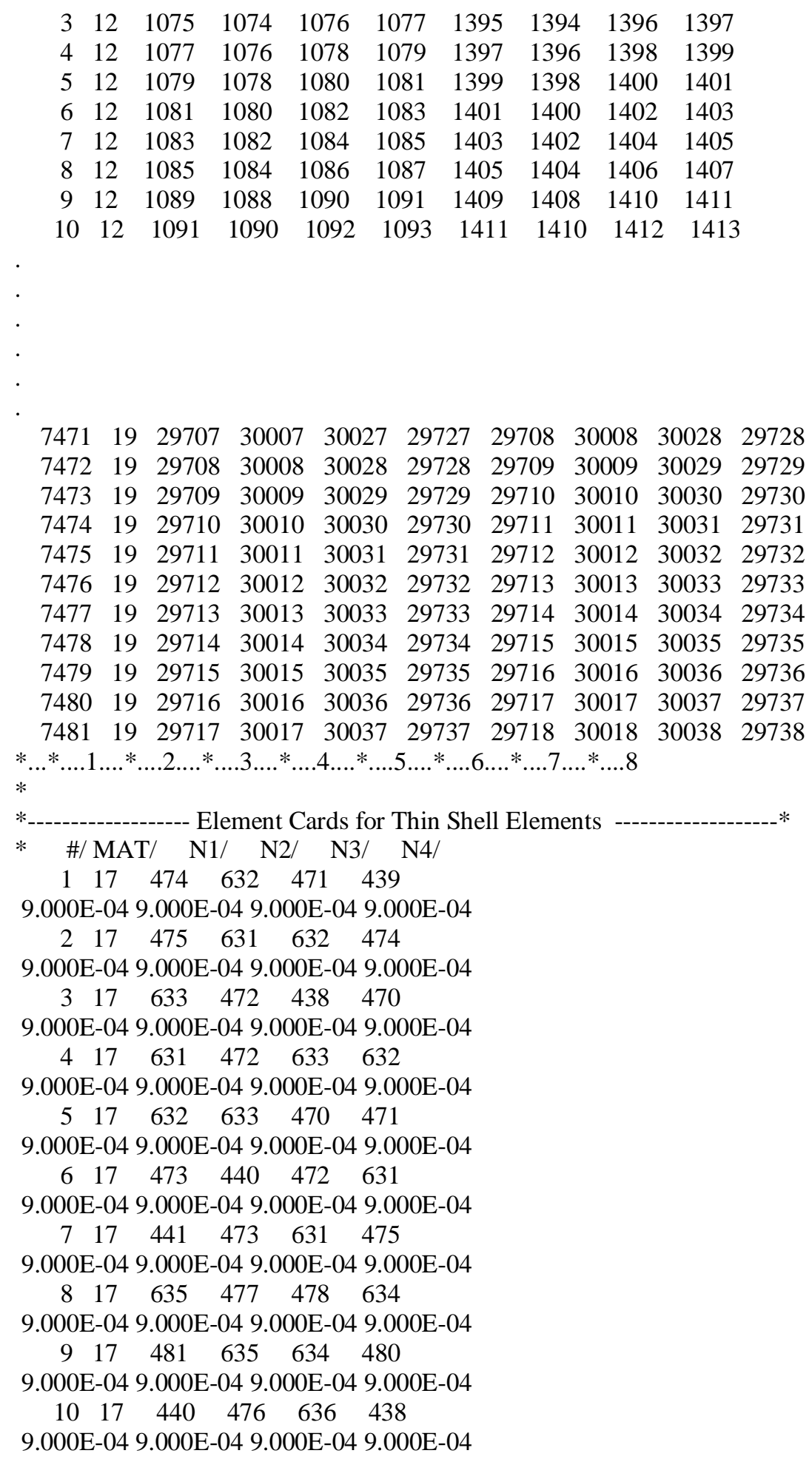


$\begin{array}{llllll}15211 & 1 & 27228 & 27571 & 27572 & 26089\end{array}$ 6.000E-03 6.000E-03 6.000E-03 6.000E-03 $\begin{array}{llllll}15212 & 1 & 27226 & 27571 & 27572 & 25438\end{array}$ 6.000E-03 6.000E-03 6.000E-03 6.000E-03 $\begin{array}{llllll}15213 & 1 & 27225 & 27571 & 27572 & 26087\end{array}$ 6.000E-03 6.000E-03 6.000E-03 6.000E-03 $\begin{array}{llllll}15214 & 1 & 27224 & 27571 & 27572 & 26087\end{array}$ 6.000E-03 6.000E-03 6.000E-03 6.000E-03 $\begin{array}{llllll}15215 & 1 & 27223 & 27571 & 27572 & 25437\end{array}$ 6.000E-03 6.000E-03 6.000E-03 6.000E-03 $\begin{array}{llllll}15216 & 1 & 27222 & 27571 & 27572 & 25437\end{array}$ 6.000E-03 6.000E-03 6.000E-03 6.000E-03 $\begin{array}{llllll}15217 & 1 & 27571 & 27220 & 26085 & 27572\end{array}$ 6.000E-03 6.000E-03 6.000E-03 6.000E-03 $\begin{array}{llllll}15218 & 1 & 27571 & 27219 & 26084 & 27572\end{array}$ 6.000E-03 6.000E-03 6.000E-03 6.000E-03 $\begin{array}{llllll}15219 & 1 & 27571 & 27218 & 25444 & 27572\end{array}$ 6.000E-03 6.000E-03 6.000E-03 6.000E-03 $\begin{array}{llllll}15220 & 1 & 27571 & 27217 & 26083 & 27572\end{array}$ 6.000E-03 6.000E-03 6.000E-03 6.000E-03 $\begin{array}{llllll}15221 & 1 & 27571 & 27215 & 25445 & 27572\end{array}$ 6.000E-03 6.000E-03 6.000E-03 6.000E-03 $\begin{array}{llllll}15222 & 1 & 27571 & 27214 & 26082 & 27572\end{array}$ 6.000E-03 6.000E-03 6.000E-03 6.000E-03 $\begin{array}{llllll}15223 & 1 & 27571 & 27213 & 25439 & 27572\end{array}$ 6.000E-03 6.000E-03 6.000E-03 6.000E-03 $\begin{array}{llllll}15224 & 1 & 27571 & 26077 & 26081 & 27572\end{array}$ 6.000E-03 6.000E-03 6.000E-03 6.000E-03

\begin{tabular}{|c|c|c|c|c|c|c|c|}
\hline \multicolumn{8}{|c|}{$\begin{array}{cccc}*---- & - \\
1 & 3 & 0 & 1.00000\end{array}$} \\
\hline \multicolumn{8}{|c|}{$0.0000000-246.00000$} \\
\hline \multicolumn{8}{|c|}{$0.5000000-246.00000$} \\
\hline \multicolumn{8}{|c|}{$1.0000000-246.00000$} \\
\hline \multirow{2}{*}{\multicolumn{8}{|c|}{$\begin{array}{l}* \text { NODAL LOAD DEFINITIONS } * * * * * \\
* \ldots * \ldots 1 \ldots * \ldots . \ldots * \ldots . \ldots * \ldots . \ldots * \ldots . \ldots \ldots * \ldots 6 \ldots * \ldots 7 \ldots * \ldots . . .\end{array}$}} \\
\hline & & & & & & & \\
\hline 275922 & 21 & 1 & 0 & 0 & 0 & 0 & 0 \\
\hline 275932 & 21 & 1 & 0 & 0 & 0 & 0 & 0 \\
\hline 275942 & 21 & 1 & 0 & 0 & 0 & 0 & 0 \\
\hline $27595 \quad 2$ & 21 & 1 & 0 & 0 & 0 & 0 & 0 \\
\hline 275962 & 21 & 1 & 0 & 0 & 0 & 0 & 0 \\
\hline $27597 \quad 2$ & 21 & 1 & 0 & 0 & 0 & 0 & 0 \\
\hline $27598 \quad 2$ & 21 & 1 & 0 & 0 & 0 & 0 & 0 \\
\hline 275992 & 21 & 1 & 0 & 0 & 0 & 0 & 0 \\
\hline 276002 & 21 & 1 & 0 & 0 & 0 & 0 & 0 \\
\hline 276012 & 21 & 1 & 0 & 0 & 0 & 0 & 0 \\
\hline 276022 & 21 & 1 & 0 & 0 & 0 & 0 & 0 \\
\hline 276032 & 21 & 1 & 0 & 0 & 0 & 0 & 0 \\
\hline 276042 & 21 & 1 & 0 & 0 & 0 & 0 & 0 \\
\hline 276052 & 21 & 1 & 0 & 0 & 0 & 0 & 0 \\
\hline 276062 & 21 & 1 & 0 & 0 & 0 & 0 & 0 \\
\hline $27607 \quad 2$ & 21 & 1 & 0 & 0 & 0 & 0 & 0 \\
\hline 276082 & 21 & 1 & 0 & 0 & 0 & 0 & 0 \\
\hline 27609 & 21 & 1 & 0 & 0 & 0 & 0 & 0 \\
\hline 276102 & 21 & 1 & 0 & 0 & 0 & 0 & 0 \\
\hline 276112 & 21 & 1 & 0 & 0 & 0 & 0 & 0 \\
\hline 276122 & 21 & 1 & 0 & 0 & 0 & 0 & 0 \\
\hline
\end{tabular}




$$
\|
$$


$100.000 \mathrm{E}+000.000 \mathrm{E}+000.000 \mathrm{E}+00$

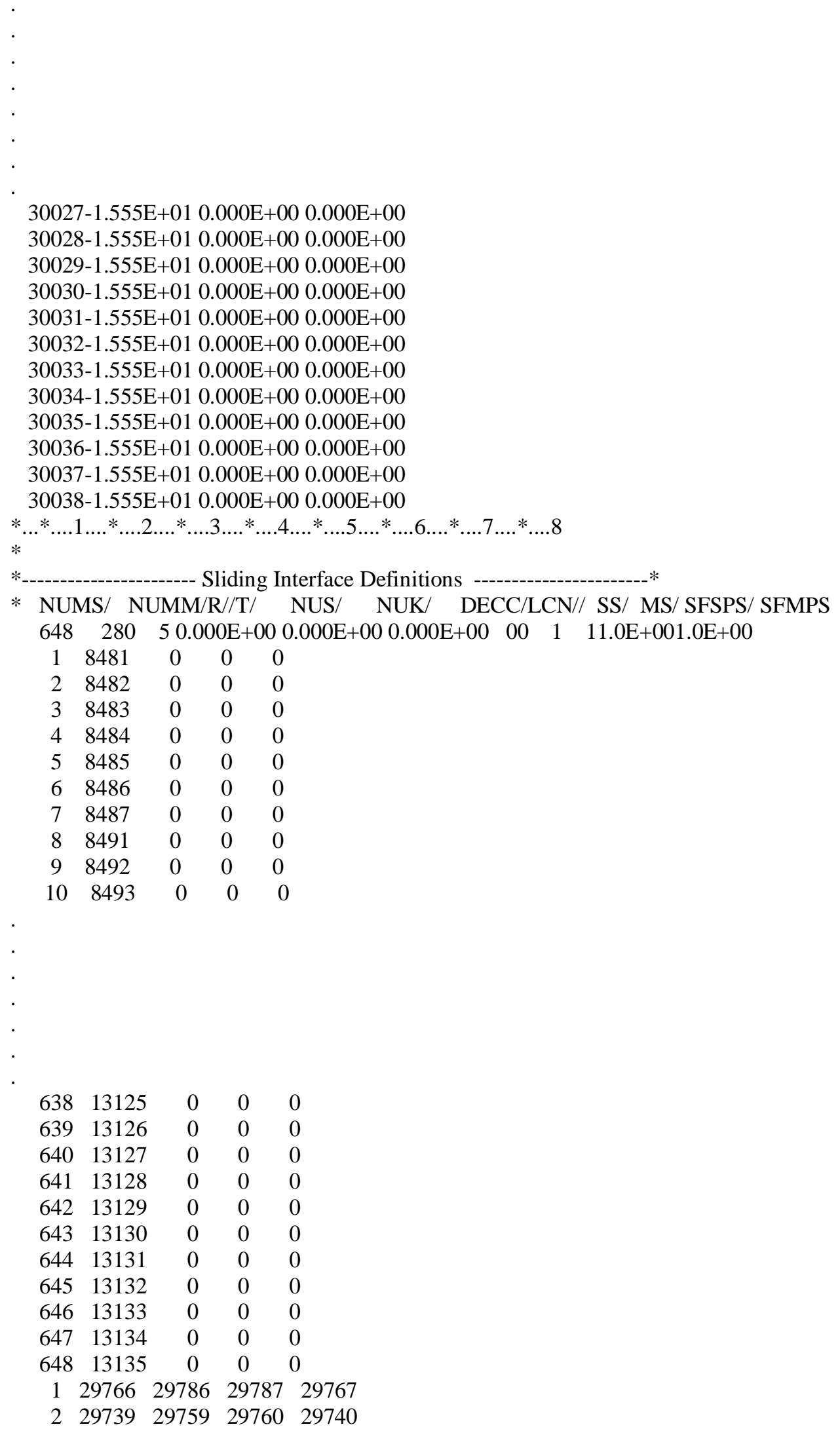


$\begin{array}{lllll}3 & 29740 & 29760 & 29761 & 29741\end{array}$

$\begin{array}{lllll}4 & 29741 & 29761 & 29762 & 29742\end{array}$

$\begin{array}{lllll}5 & 29742 & 29762 & 29763 & 29743\end{array}$

$\begin{array}{lllll}6 & 29743 & 29763 & 29764 & 29744\end{array}$

$\begin{array}{llllll}7 & 29744 & 29764 & 29765 & 29745\end{array}$

$\begin{array}{llllll}8 & 29745 & 29765 & 29766 & 29746\end{array}$

$\begin{array}{lllll}9 & 29746 & 29766 & 29767 & 29747\end{array}$

$\begin{array}{lllll}10 & 29747 & 29767 & 29768 & 29748\end{array}$

$\begin{array}{lllll}271 & 30008 & 30028 & 30029 & 30009\end{array}$

$\begin{array}{lllll}272 & 30009 & 30029 & 30030 & 30010\end{array}$

$\begin{array}{llllll}273 & 30010 & 30030 & 30031 & 30011\end{array}$

$\begin{array}{lllll}274 & 30011 & 30031 & 30032 & 30012\end{array}$

$\begin{array}{llllll}275 & 30012 & 30032 & 30033 & 30013\end{array}$

$\begin{array}{lllll}276 & 30013 & 30033 & 30034 & 30014\end{array}$

$\begin{array}{llllll}277 & 30014 & 30034 & 30035 & 30015\end{array}$

$\begin{array}{lllll}278 & 30015 & 30035 & 30036 & 30016\end{array}$

$\begin{array}{llllll}279 & 30016 & 30036 & 30037 & 30017\end{array}$

$\begin{array}{lllll}280 & 30017 & 30037 & 30038 & 30018\end{array}$ 


\section{APPENDIX C - YIELD STRESS OF MATERIALS}

\begin{tabular}{|c|c|}
\hline MATERIAL & YIELD STRESS N/m ${ }^{2}$ \\
\hline STEEL & $3.54 \times 10^{8}$ \\
\hline CAST IRON & $3.54 \times 10^{8}$ \\
\hline TIRES & $2.00 \times 10^{6}$ \\
\hline
\end{tabular}




\section{VITA}

The author Ganesh Panneer was born in Trichy, TamilNadu, India on December 9, 1973. After graduating from a high school in Trichy, he entered the Government College of Technology and received his Bachelor of Engineering degree in Mechanical Engineering in May 1995.

After graduating from Government College of Technology, he was working as a Senior Development Engineer in the CAE department for Ashok Leyland Ltd., one of the leading manufacturers of trucks and buses in India. He came to West Virginia University for the spring semester 1997 for his graduate studies in Mechanical Engineering. This thesis was written in May 1998 , as a partial fulfillment towards a degree in the Master of Science in Mechanical Engineering (M.S.M.E) at the West Virginia University. 


\section{APPROVAL OF EXAMINING COMMITTEE}

Victor H. Mucino, Ph.D.

James E. Smith, Ph.D.

Date

Gregory J. Thompson, Ph.D.

Chairman 